Sarah Schniewindt

\title{
Einführung von Wettbewerb auf der Letzten Meile
}




\section{Sarah Schniewindt}

\section{Einführung von Wettbewerb auf der Letzten Meile}

Die Telekommunikationsbranche in Deutschland hat sich seit ihrer Liberalisierung als einer der dynamischsten Märkte in vielerlei Hinsicht gezeigt. Ein Bereich jedoch, in dem die Liberalisierung keine Dynamik entfaltet hat, ist der Teilnehmeranschlußbereich, die sogenannte Letzte Meile. Dort ist die Einführung von Wettbewerb aufgrund der vertikalen Integration des ehemaligen Monopolisten erheblich erschwert worden. Ziel dieser Arbeit ist, die Problematik der ,Letzten Meile' aus technischer und netzökonomischer Sicht zu analysieren, eine Marktabgrenzung vorzunehmen sowie das Wettbewerbspotential $\mathrm{zu}$ untersuchen. Es wird sich insbesondere mit Netzzugangsregelungen theoretisch auseinandergesetzt. Zudem wird ein kurzer Einblick in die Regulierungspraxis gegeben, die aus einer politökonomischen Perspektive kritisch beleuchtet wird.

Sarah Schniewindt studierte von 1991 bis 1996 Volkswirtschaftslehre und Politologie an der Freien Universität Berlin. Von 1997 bis 2002 war sie als wissenschaftliche Mitarbeiterin am Institut für Theoretische Volkswirtschaftslehre der Universität der Bundeswehr Hamburg tätig, wo sie im Jahr 2002 promoviert wurde. 
Einfuhrung von Wettbewerb auf der Letzten Meile 


\title{
SCHRIFTEN ZUR \\ WIRTSCHAFTSTHEORIE UND WIRTSCHAFTSPOLITIK
}

\author{
Herausgegeben von \\ Rolf Hasse,Jörn Kruse, Wolf Schäfer, Thomas Straubhaar \\ und KlausW.Zimmermann
}

Band 28

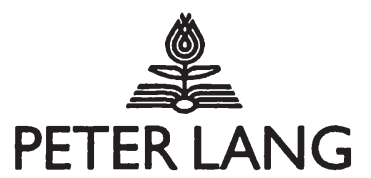

Frankfurt am Main - Berlin - Bern - Bruxelles - NewYork - Oxford - Wien 


\section{Sarah Schniewindt}

\section{Einführung von Wettbewerb auf der Letzten Meile}

Eine ökonomischeAnalyse von Netzinfrastruktur und Wettbewerbspotential im Teilnehmeranschlußbereich

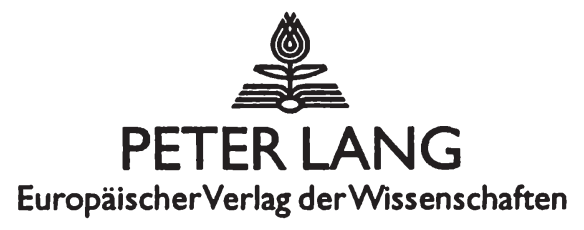


Bibliografische Information Der Deutschen Bibliothek

Die Deutsche Bibliothek verzeichnet diese Publikation in der

Deutschen Nationalbibliografie; detaillierte bibliografische

Daten sind im Internet uber <http://dnb.ddb.de> abrufbar.

Open Access: The online version of this publication is published on www.peterlang.com and www.econstor.eu under the international Creative Commons License CC-BY 4.0. Learn more on how you can use and share this work: http://creativecommons.org/ licenses/by/4.0.

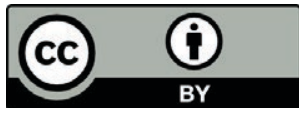

This book is available Open Access thanks to the kind support of ZBW - Leibniz-Informationszentrum Wirtschaft.

Zugl.: Hamburg, Univ. der Bundeswehr, Diss., 2002

Gedruckt mit Unterstützung der Bundeswehr Hamburg.

Gedruckt auf alterungsbeständigem, säurefreiem Papier.

\author{
D705 \\ ISSN 1433-1519 \\ ISBN3-631-51004-7 \\ ISBN 978-3-631-75130-5 (eBook) \\ (C) Peter Lang GmbH \\ Europäischer Verlag der Wissenschaften \\ Frankfurt am Main 2003 \\ Alle Rechte vorbehalten.
}

Das Werk einschließlich aller seiner Teile ist urheberrechtlich geschützt. Jede Verwertung außerhalb der engen Grenzen des

Urheberrechtsgesetzes ist ohne Zustimmung des Verlages unzulässig und strafbar. Das gilt insbesondere für

Vervielfältigungen, Übersetzungen, Mikroverfilmungen und die Einspeicherung und Verarbeitung in elektronischen Systemen.

Printed in Germany 123457

www.peterlang.de 


\section{FÜR TILl, ARThUR UND Gregor}


Sarah Eliza Schniewindt - 978-3-631-75130-5 Downloaded from PubFactory at 01/11/2019 07:57:00AM via free access 


\section{VORWORT}

Die vorliegende Arbeit entstand während meiner Zeit als wissenschaftliche Mitarbeiterin am Institut fur Theoretische Volkswirtschaftslehre an der Universität der Bundeswehr Hamburg. Bei ihrer Entstehung habe ich vielfalltige Unterstützung erfahren, für die ich mich an dieser Stelle bedanken möchte.

An erster Stelle möchte ich mich bei meinem Doktorvater Prof. Dr. Wolf Schaffer bedanken, der mit konstruktiver Kritik und zahlreichen Diskussionen wesentlich zum Gelingen dieser Arbeit beigetragen hat. Vor allem aber gewährte er mir die nötigen Freirăume für die Fertigstellung der Arbeit. Prof. Dr. Jörn Kruse übernahm nicht nur das Zweitgutachten, sondern er begleitete das Entstehen der Arbeit. Ich bin ihm um manche Anregung und Kritik dankbar. Prof. Dr. Klaus W. Zimmermann danke ich für seine Mitwirkung an meinem Promotionsverfahren. Prof. Dr. Gudrun Peschutter nahm aktiv am Entstehen der Arbeit Anteil. Ich danke ihr vor allem für die aufmunternden Worte zu Beginn. Dr. Silvia Rottenbiller und Babette Hagemann lasen Teile der Arbeit kritisch Korrektur. Ihnen danke ich für die hilfreichen Diskussionen und Anmerkungen sowie meinen Kollegen Annette Olbrisch, Dr. Malgorzata Stankiewicz, die mir mit Rat und Tat Beistand leisteten und Dr. Gerhard Rösl sowie Christoph Kimmel, die so manchen Seufzer erduldeten.

Vor allem aber möchte ich mich bei Dr. Till Hoja bedanken, der mich über die Jahre der Entstehung motiviert, moralisch unterstützt und gestutzt hat. Ihm und unseren beiden Kindern widme ich diese Arbeit. Sie gaben mir neben dem wissenschaftlichen Arbeiten die nötige Bodenhaftung und letztendlich viel Kraft. Außerdem möchte ich mich ganz besonders bei Wiete Hoja bedanken, die mir zur Seite stand, indem sie immer für unsere Kinder da war. So auch Dr. HansChristian Hoja und meine Eltern, Wolfgang und Marina Schniewindt sowie Klaus und Erika Evers und Krista Weist, bei denen ich Arthur und Gregor immer gut aufgehoben wußte. Ohne ihre Hilfe hătte ich dieses Buch nie fertigstellen können.

Schließlich möchte ich der Universität der Bundeswehr für die finanzielle Unterstultzung bei der Drucklegung danken.

Hamburg, im Dezember 2002

Sarah Schniewindt 
Sarah Eliza Schniewindt - 978-3-631-75130-5 Downloaded from PubFactory at 01/11/2019 07:57:00AM via free access 


\section{INHALTSVERZEICHNIS}

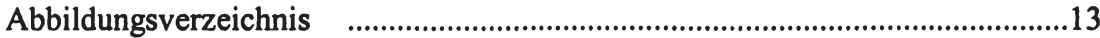

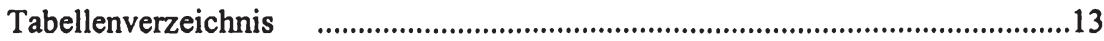

Abkürzungsverzeichnis $\quad$...........................................................................14

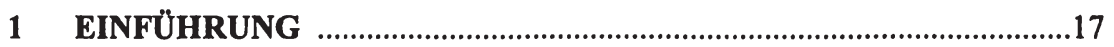

2 CHARAKTERISIERUNG DES ORTS- UND

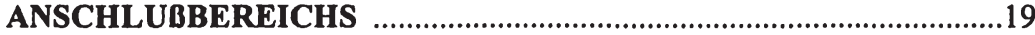

2.1 Einfuhrung in die technischen Grundlagen .......................................19

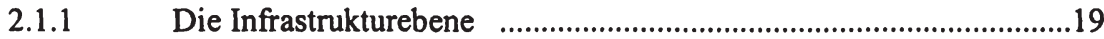

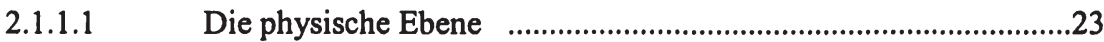

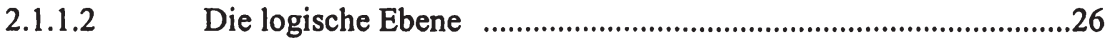

2.1.1.3 Die technische Weiterentwicklung .............................................27

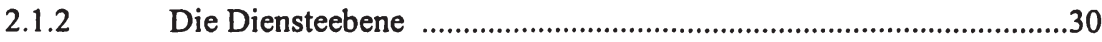

2.2 Einfuhrung in die wettbewerbspolitische Problemstellung für den Orts- bzw. Anschlußbereich …...........................................................31

2.3 Die Teilnehmeranschlußleitung als natürliches Monopol ...................33

2.3.1 Eigenschaften eines natürlichen Monopols .........................................35

2.3.2 Dynamische Aspekte natürlicher Monopole .....................................38

2.3.3 Ökonomische Konsequenzen der Digitalisierung ............................41

2.3.4 Fazit fur die Entwicklung von Wettbewerb im Teilnehmeranschlußbereich _............................................................45

2.4 Bewertung institutioneller Rahmenbedingungen fur die

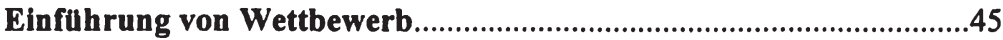

2.4.1 Beseitigung institutioneller Markteintrittsbarrieren .........................47

2.4.2 Gewährung von Netzzugang …....................................................48

2.4.2.1 Regelung des Netzzugangs .....................................................48

2.4.2.2 Entbündelung von Netzelementen insbesondere der

2.4.2.3 Bewertung der Netzzugangsregelung …...................................52

2.4.3 Zusammenschaltung (Interconnection) ..........................................53

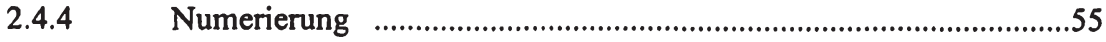

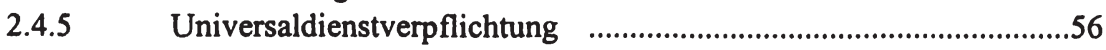

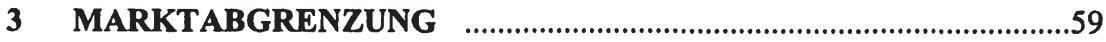

3.1 Wettbewerbsformen des Markteintritts ...........................................59

3.1.1 Wiederverkäuferwettbewerb („Resale Competition“) .......................60

3.1.2 Dienstewettbewerb (,Service Competition“) ..................................61 
3.1.3 Infrastrukturbasierter Wettbewerb

(,Facilities Based Competition") ..................................................61

3.2 Kriterien der Marktabgrenzung und Marktstruktur ........................65

3.2.1 Der Vergleichsmarktansatz .......................................................65

3.2.2 Substitutionskonzepte zur sachlichen Marktabgrenzung .................66

3.2.3 Das Konzept des hypothetischen Monopolisten .............................68

3.2.3.1 Nachfragesubstitution ……......................................................70

3.2.3.2 Angebotssubstitution ...............................................................

3.2.3.3 Potentieller Wettbewerb ……….............................................72

3.3 Die Abgrenzung des relevanten Marktes für infrastrukturbasierten Wettbewerb ......................................................73

3.3.1 Markt für Festnetzdienste ……....................................................73

3.3.2 Marktabgrenzung nach dem Konzept des hypothetischen

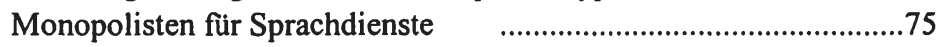

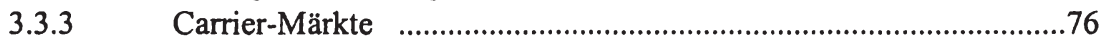

3.3.3.1 Zusammenschaltungsleistungen von Festnetzanschlüssen $\quad . . . . . . . . .77$

3.3.3.2 Zugang zu Teilnehmeranschlußleitungen ...................................78

3.3.3.3 Mietleitungen fur Netzbetreiber .................................................79

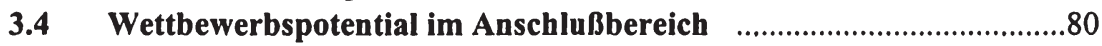

3.4.1 Netzbasierte Anschlußtechnologien .................................................82

3.4.1.1 Das TV-Breitbandkabel (CA-TV) …........................................82

3.4.1.2 Datenübertragung über Stromnetze …........................................83

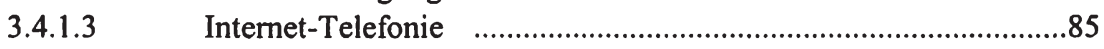

3.4.2 Funkgestützte Anschlußtechnologien …..........................................88

3.4.2.1 Richtfunk ( Wireless Local Loop ) ..............................................8

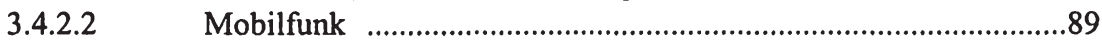

3.4.3 Marktabgrenzung alternativer Anschlußtechnologien zur

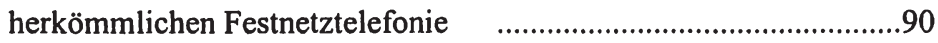

3.4.3.1 Nachfragesubstitutionsbereitschaft ….........................................91

3.4.3.2 Angebotssubstitutionsmöglichkeiten .......................................99

3.4.3.3 Potentieller Wettbewerb ….........................................................96

3.4.4 Vertikal sachliche Marktabgrenzung ...............................................100

4 ÖKONOMISCHE GRUNDLAGEN VON

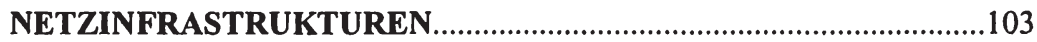

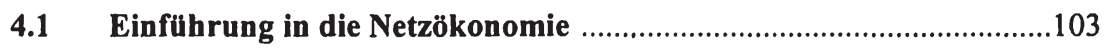

4.1.1 Klassifikation von Netzeffektgütern .............................................103

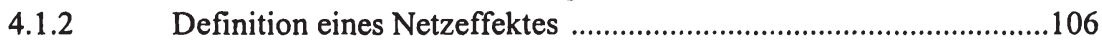

4.1.3 Definition von Netzexternalitäten ................................................100

4.1.4 Möglichkeiten der Internalisierung von Netzexternalitäten ............108 


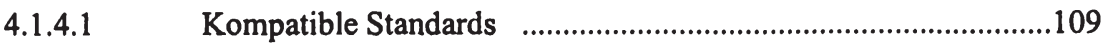

4.1.4.2 Inkompatible Standards $\quad$..........................................................111

4.1.4.3 Bewertung von Standards ……...........................................112

4.2 Zusammenschaltung von Netzen ……..............................................114

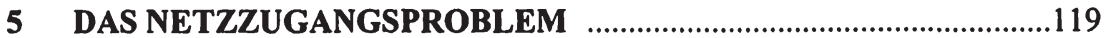

5.1 Allgemeine Darstellung von Preisregeln …........................................120

5.1.1 Die Grenzkostenpreisregel ......................................................121

5.1.2 Die Durchschnittskostenpreisregel bei einem Starken Natürlichen Monopol..................................................................122

5.1.3 Preisregel auf der Grundlage von inkrementellen Kosten ..............122

5.1.4 Stand-Alone Kosten ................................................................124

5.1.5 Die Ramsey - Preisregel …............................................................125

5.1.6 Die ,Efficient Component Pricing Rule` ......................................130

5.2 Möglichkeiten der Festlegung eines Zugangsentgelts bei einem

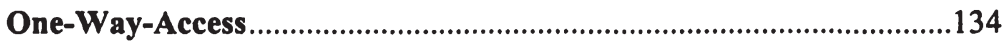

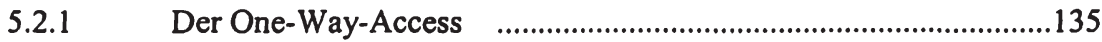

5.2.1.1 Der Wiederverkäuferwettbewerb ….......................................135

5.2.1.2 Der Dienstewettbewerb …...................................................138

5.2.2 Ermittlung eines Zugangsentgelts nach den Grenzkosten bzw. nach den inkrementellen Kosten............................................139

5.2.3 Ermittlung eines Zugangsentgelts nach der Ramsey- Preisregel ....140

5.2.4 Ermittlung eines Zugangsentgelts nach der ECPR ........................136

5.2.5 Ermittlung eines Zugangsentgelts bei abhängigen Nachfragen .....143

5.2.6 Fazit: Vergleich der Preisregeln im Hinblick auf die Einführung von Wettbewerb...............................................................148

5.3 Verhandlungen von Zusammenschaltungsentgelten bei einem Two-Way-Access ......................................................................................149

5.3.1 Bertrand- Wettbewerb bei vollständiger Substituierbarkeit …......150

5.3.2 Bertrand- Wettbewerb bei horizontaler Produktdifferenzierung ...157

5.3.2.1 Modellierung der Marktanteile …............................................158

5.3.2.2 Horizontale Produktdifferenzierung f...........................................161

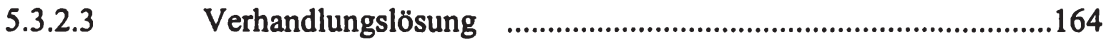

5.3.2.4 Wirtschaftspolitische Empfehlung ….........................................167

5.4 Kostenorientierte Rechnungsansătze in der Regulierungspraxis ....169

5.4.1 Das kostenorientierte Regulierungsverfahren ...............................170

5.4.2 Der Top-Down- Ansatz …….....................................................173

5.4.3 Der Bottom-Up- Ansatz …......................................................175

5.4.3.1 Bottom-Up- Kostenmodelle in der Praxis $\quad$.................................177

5.4.3.2 Bewertung des Bottom-Up- Ansatzes _......................................180 
5.4.4 Gemeinkostenallokation bei kostenorientierten Rechnungsansätzen 182

5.4.4.1 Das Fully Distributed Cost Pricing- Verfahren ..........................183

5.4.4.2 Das Shapley-Value- Verfahren ………....................................184

5.4.5 Kritik an kostenorientierten Rechnungsansätzen bzw. an der Orientierung an langfristigen zusätzlichen Inkrementalkosten ......185

6 POLITÖKONOMISCHE SICHTWEISE _..........................................191

6.1 Themenrelevante Grundzuge der Prinzipal-Agent- Theorie ...........192

6.2 Prinzipal, Agentur und Agent in der Telekommunikation ...............194

6.2.1 Verhaltenskalkül der Agentur ......................................................196

6.2.2 Verhaltenskalkül des Agenten ................................................198

6.2.3 Einfluß von Interessengruppen .................................................198

6.3 Strategische Interaktionen zwischen Prinzipal, Agentur und Agent in der Telekommunikation ......................................................200

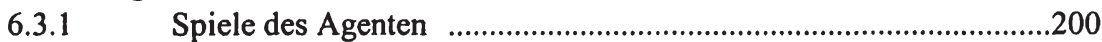

6.3.2 Spiele des Wettbewerbers ….......................................................201

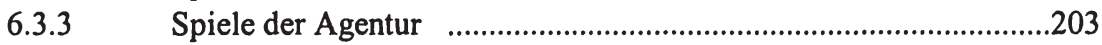

6.3.4 Fazit und Bewertung f..........................................................204

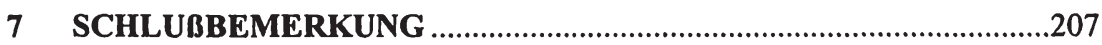

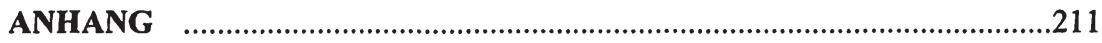

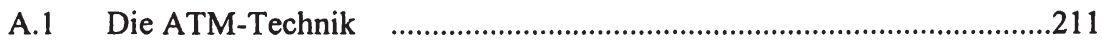

A.2 Bestimmung von Haupt- und Unterdeterminante $\quad$...........................213

A.3 Monopolpreise bei sehr hohem Produktdifferenzierungsgrad ................215

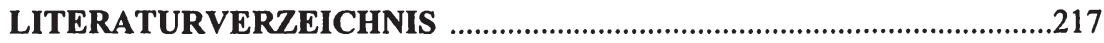




\section{ABBILDUNGSVERZEICHNIS}

Abbildung 2.1.-1: Struktur des digitalen Fernsprechnetzes ...............................20

Abbildung 2.1.-2: Architektur eines Ortsnetzes mit Kupferdoppeladern .........22

Abbildung 2.1.-3: Sektorstruktur in der Telekommunikation ............................30

Abbildung 2.3.-1: ,Economies of Scale ' und Natürliches Monopol ..................37

Abbildung 2.3-2: Erosion eines Natürlichen Monopols durch Nachfrageausweitung .......................................................................39

Abbildung 2.3.-3: Erosion eines Natürlichen Monopols durch technischen

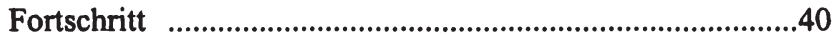

Abbildung 2.3.-4: Folgen der Digitalisierung im Festnetzanschlußbereich ....41

Abbildung 3.2.-1: Zielsetzung und Instrumentarium der Marktabgrenzung .....69

Abbildung 4.2.-1: Darstellung der Höhe von Netzexternalităten ...................116

Abbildung 5.2.-1: Wiederverkăuferwettbewerb ……………………….........136

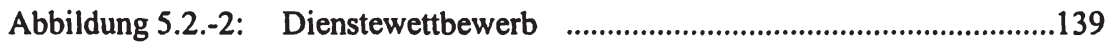

Abbildung 5.3.-1: Infrastrukturbasierter Wettbewerb …..................................151

Abbildung 5.3.-2: Aufteilung der Telefongespräche in on-net und

off-net- Dienste $\quad$...............................................................158

\section{TABELLENVERZEICHNIS}

Tabelle 2.1.-1: $\quad$ Merkmale einzelner Übertragungsmedien...............................24

Tabelle 2.1.-2: $\quad$ X- DSL- Übertragungsvarianten im Vergleich.......................29

Tabelle 3.4.-1: $\quad$ Formen der Internet Telefonie..................................................87

Tabelle 3.4-2: $\quad$ Horizontale Marktabgrenzung lokaler Anschlußtechnologien zur herkömmlichen Festnetztelefonie................91

Tabelle 3.4.-3: $\quad$ Kriterien der Nachfragesubstitutionsbereitschaft hinsichtlich lokaler Anschlußtechnologien im Vergleich zur

Festnetztelefonie .92

Tabelle 3.4-4: $\quad$ Zentrale Elemente furr Markteintritt potentieller Wettbewerber .96

Tabelle 3.4.-5: Vertikale sachlich relevante Marktabgrenzung lokaler Anschlußtechnologien .101 


\section{ABKÜRZUNGSVERZEICHNIS}

\begin{tabular}{|c|c|}
\hline ADSL & - High Bit Rate Digital Subscriber Line \\
\hline ATD & - Asynchronous Time Division \\
\hline ATM & - Asynchronous Transfer Mode \\
\hline BTX & - Bildschirmtext \\
\hline CA-TV & - Cable-Television \\
\hline C-C-IT & - Computer zu Computer Internet Telefonie \\
\hline C-T-IT & - Computer zu Telefon Internet Telefonie \\
\hline DIK & - Durchschnittliche Inkrementalkosten \\
\hline DTAG & - Deutsche Telekom AG \\
\hline EBC & - Element Based Charging \\
\hline ECPR & - Efficient Component Pricing Rule \\
\hline ETSI & - European Telecommunications Standard Institute \\
\hline EVU & - Elektrizitätsversorgungsunternehmen \\
\hline Evz & - Endverzweiger \\
\hline FCC & - Federal Commission of Communication \\
\hline FL LRAIC & - Forward Looking Long Run Average Incremental Costs \\
\hline FL LRIC & - Forward Looking Long Run Incremental Costs \\
\hline GAN & - Global Area Network \\
\hline GSM & - Group Special Mobile \\
\hline GWB & - Gesetz gegen Wettbewerbsbeschränkungen \\
\hline HAI & - Hatfield Kostenmodell \\
\hline HCPM & - Hybrid Cost Proxy Models \\
\hline HDSL & - High Bit Rate Digital Subscriber Line \\
\hline Hk & - Hauptkabel \\
\hline HVT & - Hauptverteiler \\
\hline $\mathrm{Hz}$ & - Hertz \\
\hline IDN & - Integrated Data Network \\
\hline IEEE & - Institute of Electrical and Electronic Engineers \\
\hline IK & - Inkrementalkosten \\
\hline IP & - Internet Protocol \\
\hline ISDN & - Integrated Services Digital Network \\
\hline ISO & - International Standard Organisation \\
\hline ISP & - Internet Service Provider \\
\hline IT & - Internet Telefonie \\
\hline ITSP & - Internet Service Provider \\
\hline ITU & - International Telecommunications Network \\
\hline $\mathrm{K}$ & - abgesetzter Konzentrator \\
\hline $\mathrm{kbit} / \mathrm{s}$ & - kilobit pro Sekunde \\
\hline
\end{tabular}




$\begin{array}{ll}\text { kHz } & \text { - kilo Hertz } \\ \text { kV } & \text { - kiloVolt } \\ \text { KVSt } & \text { - Knotenvermittlungsstelle } \\ \text { Kvz } & \text { - Kabelverzweiger } \\ \text { LAN } & \text { - Local Area Network } \\ \text { LDK } & \text { - Langfristige Durchschnittskosten } \\ \text { LRIC } & \text { - long run incremental costs } \\ \text { LWL } & \text { - Lichtwellenleiter } \\ \text { MAN } & \text { - Metropolitan Area Network } \\ \text { Mbit/s } & \text { - Megabit pro Sekunde } \\ \text { NZV } & \text { - Netzzugangsverordnung } \\ \text { o.a. } & \text { - overlay approach } \\ \text { Oftel } & \text { - Office of Telecommunications } \\ \text { OSI } & \text { - Open System Interconnection } \\ \text { OVSt } & \text { - Ortsvermittlungsstelle } \\ \text { PCM } & \text { - Puls Code Modulation } \\ \text { PLC } & \text { - Powerline Communication } \\ \text { PMP-Rifu } & \text { - Punkt zu Mehrpunkt Richtfunk } \\ \text { POTS } & \text { - Plain Old Telephone System/Service } \\ \text { PSTN } & \text { - Public Switched Telephone Network } \\ \text { PTM } & \text { - paketorientierter Transfermodus } \\ \text { RegTP } & \text { - Regulierungsbehörde fur Telekommunikation und Post } \\ \text { RVSt } & \text { - Regionalvermittlungstelle } \\ \text { SAK } & \text { - Stand Alone Kosten } \\ \text { SDH } & \text { - Synchron Digital Hierarchy } \\ \text { TAL } & \text { - Teilnehmeranschlußleitung } \\ \text { TCP } & \text { - Transmission Control Protocol } \\ \text { TELRIC } & \text { - Total Element Long Run Incremental Cost } \\ \text { TentgV } & \text { - Telekommunikations-Entgeltregulierungsverordnung } \\ \text { TFH } & \text { - Trägerfrequenztechnik auf Hochspannungsleitungen } \\ \text { TIME } & \text { - Telecommunications, Information, Media, Entertainment, } \\ & \text { Electronics } \\ \text { TK } & \text { - Totale Kosten } \\ \text { TKG } & \text { - Telekommunikationsgesetz } \\ \text { TRT } & \text { - Tonfrequenz- Rundsteuertechnik } \\ \text { T-T-IT } & \text { - Telefon zu Telefon Internet Telefonie } \\ \text { t.o.c. } & \text { - telephony over coax } \\ \text { UDP } & \text { - User Datagram Protocol } \\ \text { UMTS } & \text { - Universal Mobile Communications } \\ \text { VDSL } & \text { - Very High Bit Rate Digital Subscriber Line } \\ & \end{array}$


VoIP

Vzk

WAN

WIK

WLL

WVSt
- Voice over Internet Protocol oder auch Internet Telefonie

- Verzweigerkabel

- Wide Area Network

- Wissenschaftliches Institut für Kommunikationsdienste

- Wireless Local Loop

- Weitverkehrsvermittlungsstelle 
Die Telekommunikationsbranche in Deutschland hat sich seit ihrer Liberalisierung im Jahr 1998 als einer der dynamischsten Märkte in vielerlei Hinsicht gezeigt, sowohl was die zunehmende Zahl an Wettbewerbern, die Entwicklung des Umsatzwachstums als auch die Diffusion technischer Innovationen betrifft. Und nicht zuletzt stellen die resultierenden dramatischen Preissenkungen insbesondere auf dem Markt für Fern- und Auslandgespräche einen großen Vorteil für den Konsumenten dar. Ein Bereich der Telekommunikation jedoch, in dem die Liberalisierung des Marktes keine Dynamik entfaltet hat, ist der Teilnehmeranschlußbereich, die sogenannte, Letzte Meile‘.

Die Besonderheit der Telekommunikationsbranche ist, daß sie sich vor der Liberalisierung als ein Markt mit einem vorherrschenden Anbieter, dem ehemaligen staatlichen Monopolisten, darstellte. Erst mit der Liberalisierung in der zweiten Hälfte der 90er Jahre wurde das Bestehen von unterscheidbaren vor- und nachgelagerten Märkten offenbar. Während auf nachgelagerten Märkten die sogenannten Endprodukte gehandelt werden, also die Telekommunikationsdienste, die der Kunde in Anspruch nimmt, sind die vorgelagerten Märkte als Input-Märkte zu verstehen. Dazu zählen neben Vorleistungen vor allem die Bereitstellung der Netzinfrastruktur, welche die Entfernungen zwischen den Kunden überbrückt. Die Abhängigkeiten zwischen den beiden Marktsektoren weisen Charakteristika der Netzökonomie auf.

Bei der Liberalisierung der Telekommunikationsbranche wurde ursprunglich indes weder das Bestehen von noch die Abhängigkeiten zwischen vor- und nachgelagerten Märkten genügend bedacht. So wurde eine vertikale Integration des ehemaligen Monopolisten begünstigt, wodurch die Einfuhrung von Wettbewerb vor allem auf den vorgelagerten Märkten erheblich erschwert worden ist. Nachdem schließlich die Abhängigkeiten des Endproduktmarktes von der Netzinfrastruktur offenbar geworden waren, sollte der Einführung von Wettbewerb mit Hilfe von regulatorischen Maßnahmen Vorschub geleistet werden, frei nach dem Grundsatz: ,Deregulierung durch Regulierung'. Neuen Wettbewerbern sollte der Markteintritt gegenuber dem ehemals bestehenden Monopolisten durch Regelung von Zugängen zum Netz des Monopols erleichtert oder uberhaupt erst möglich gemacht werden. Insbesondere in dem nachgelagerten Markt fur Fern- und Auslandsgesprăche ist es ausgesprochen schnell zu vielen Markteintritten und hohen Preissenkungen gekommen. Dies erscheint zunăchst wie ein Erfolg und eine Bestătigung der Methode ,Deregulierung durch Regulierung'. Dem entgegen steht jedoch das eklatante Fehlen jedweder Dynamik auf dem Markt für Ortsgespräche und Teilnehmeranschlüsse. Hier muß man seit der Liberalisierung im Grunde von einem Stillstand der Entwicklungen sprechen. Nur in Ballungsregionen hat es eine 
geringe Anzahl an Markteintritten gegeben. Insgesamt hatten Wettbewerber deutschlandweit im Jahr 2001 einen Marktanteil von nicht einmal 3\% im Teilnehmeranschlußbereich. Dementsprechend sind die Preise für Ortsgespräche und Teilnehmeranschlüsse unverändert hoch.

Es lassen sich vielfaltige Gründe aufführen, warum sich bislang kein Wettbewerb auf dem Markt der Letzten Meile entwickelt hat. Hat sich hier die ,Liberalisierung durch Regulierung' nicht bewährt? Sollte verstärkt regulatorisch in den Markt eingegriffen werden oder sollten im Gegenteil die regulierenden Maßnahmen eingeschränkt werden? Ziel dieser Arbeit ist, eine eingehende Analyse der Problematik der ,Letzten Meile' vorzunehmen und zu untersuchen, warum sich dort bislang so geringer Wettbewerb ausgebildet hat und unter welchen Bedingungen dort Wettbewerb entstehen kann.

Hierzu wird zunächst in Kapitel 2 eine Charakterisierung des Orts- und Teilnehmeranschlußbereichs vorgenommen. Nach einer Einführung in die technischen Grundlagen läßt sich eine wettbewerbspolitische Problemstellung formulieren. Im weiteren Verlauf sollen die ökonomischen Aspekte der Letzten Meile herausgearbeitet werden, bevor die institutionellen Rahmenbedingungen vorgestellt und hinsichtlich ihrer Funktion für die Einfuihrung von Wettbewerb beurteilt werden. Um das Wettbewerbspotential substitutiver Anbieter beurteilen zu können, wird vorausgehend in Kapitel 3 eine Marktabgrenzung vorgenommen. Dazu werden zunächst die verschiedenen Wettbewerbsformen erläutert. Darauf folgt eine Hinfuhrung zu sachlichen Marktabgrenzungskonzepten. Im Vordergrund dabei steht das Konzept des ,hypothetischen Monopolisten', das im folgenden auf den infrastrukturbasierten Wettbewerb und schließlich auf substitutive Anbieter im Teilnehmeranschlußbereich angewendet wird. In Kapitel 4 wird eine Einfuhrung in die ökonomischen Grundlagen von Netzinfrastrukturen gegeben und eine ökonomische Bewertung der Zusammenschaltung von Netzen vorgenommen. Kapitel 5 beschäftigt sich ausführlich mit der Netzzugangsproblematik. Hierbei werden zum einen verschiedene Preisregeln angewendet, die den Zugang zum Netz des ehemaligen Monopolisten regeln sollen, und hinsichtlich ihrer Effizienz sowie ihrer Fähigkeit, Wettbewerb zu schaffen, bewertet. Desweiteren soll für den Fall, daß Wettbewerb im vorgelagerten Markt besteht, auf die Probleme bei Verhandlungen von reziproken Zugangsentgelten bzw. Zusammenschaltungsentgelten eingegangen werden. Nach einer theoretischen Auseinandersetzung mit dem Zugangsproblem wird zudem noch ein Einblick in die Regulierungspraxis gegeben, welche im abschließenden Kapitel 6 mit Hilfe des Prinzipal-Agent- Ansatzes aus einer politökonomischen Perspektive kritisch hinterfragt wird. In Kapitel 7 werden die Kernaussagen noch einmal kurz zusammengefaßt und ein Resümee aus der Arbeit gezogen. 


\section{2 \\ Charakterisierung des ORTS- UND ANSCHLUß- BEREICHS}

Da die Etablierung von Wettbewerb insbesondere im Teilnehmeranschlußbereich als problematisch einzustufen ist, soll den Ursachen hierfür näher auf den Grund gegangen werden. Zunächst wird der Leser in die telekommunikationsspezifischen technischen Grundlagen eingewiesen bevor die wettbewerbspolitische Problemstellung für den Teilnehmeranschlußbereich formuliert wird und auf seine ökonomischen Spezifika insbesondere eingegangen wird sowie die institutionellen Rahmenbedingungen aus wettbewerbspolitischer Sicht bewertet werden.

\subsection{EINFÜHRUNG IN DIE TECHNISCHEN GRUNDLAGEN}

In der Telekommunikation gibt es Märkte, die in einer vertikalen Beziehung zueinander stehen. Die für das Gut Telekommunikation - im einfachsten Fall ein Telefongespräch - notwendige Netzinfrastruktur stellt den vorgelagerten Markt oder auch den Inputmarkt dar. Das Telefongespräch selbst ist als Endprodukt dem nachgelagerten Markt für Telekommunikationsdienste zuzuordnen. Im Folgenden wird eine Unterteilung der Analyse in eine Infrastrukturebene (Kap. 2.1.1) und eine Diensteebene (Kap. 2.1.2) vorgenommen. Dabei ist die Infrastrukturebene der Diensteebene vorgelagert. Innerhalb der Infrastrukturebene sind die verschiedenen Netzebenen hierarchisch strukturiert.

\subsubsection{DIE INFRASTRUKTUREBENE}

Das Telekommunikationsnetz hat einen hierarchischen Aufbau aufeinander abgestimmter Netzbereiche. Die Netzbereiche werden grob in der Regel in ein Ortsund ein Fernnetz unterteilt und als getrennte Märkte betrachtetet', die in einer vertikalen Beziehung zueinander stehen.

Wie an der Abbildung 2.1-1 zu sehen ist, umfaßt das Ortsnetz die Teilnehmeranschlüsse und die Ortsvermittlungsstelle (OVSt), die lokale Anschlüsse miteinander verbindet. Das Ortsnetz ist von der logischen Struktur her wie ein Sternnetz aufgebaut und ist dem Fernnetz vorgelagert.

$\mathrm{Zu}$ einer ausfuhrlichen Marktabgrenzung vgl. Kap. 3.3. 


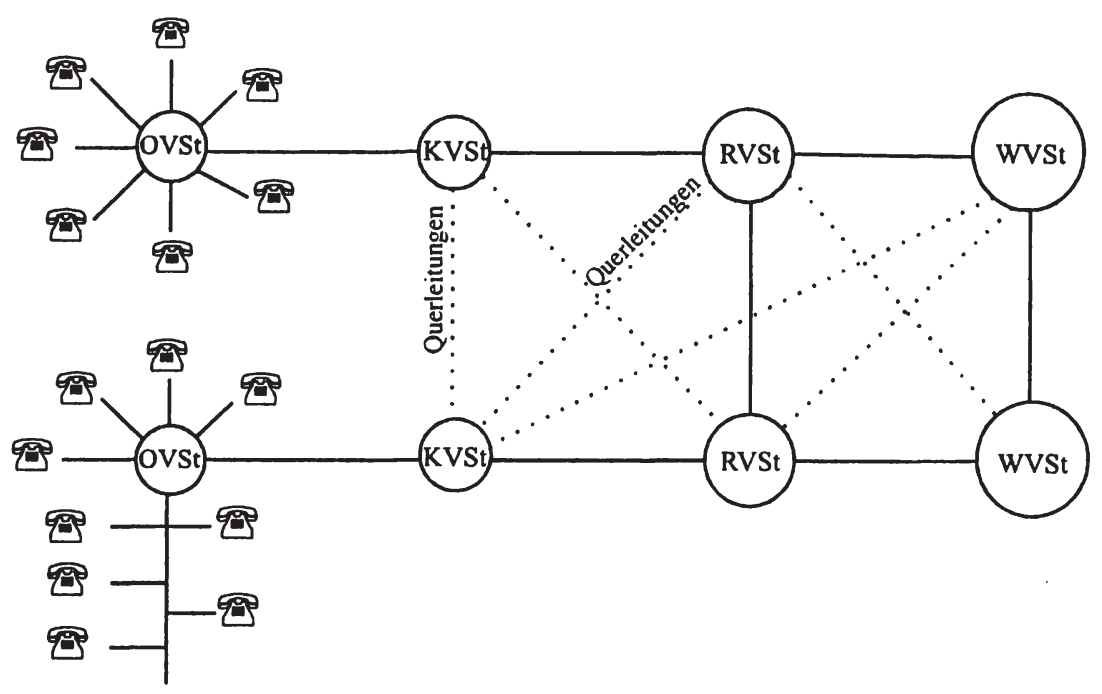
OVSt - Ortsvermittlungsstelle
KVSt - Knotenvermittlungsstelle
RVSt - Regionalvermittlungsstelle
WVSt - Weitverkehrsvermittlungsstelle

\section{Abbildung 2.1-1: Struktur des digitalen Fernsprechnetzes ${ }^{2}$}

Sternnetze werden in der Regel in unteren Netzebenen verwendet, also für das Teilnehmeranschlußleitungsnetz auf der Ortsnetzebene. Ein sternförmiges Netz ist durch einen zentralen Netzknoten - die OVSt -, uber den alle Endknoten miteinander in Verbindung stehen, gekennzeichnet. Endknoten sind die Endanschlüsse der Netzteilnehmer. Für eine Verbindung zwischen zwei Endknoten sind zwei Verbindungsleitungen notwendig, nämlich von dem einen Anschluß zum zentralen Netzknoten und von dem wiederum zu dem anderen Anschluß. Die Netzknoten sind nicht gleichrangig zu bewerten. Die Zentrale ist gegenüber den Endknoten höherrangig. Daraus ergibt sich eine hierarchische Netzstruktur.

Ein Sternnetz hat den Vorteil, daß aufgrund der Konzentration und der Vermittlung über eine Zentrale relativ niedrige Übertragungskosten entstehen. Aufgrund der zentralen Steuerung treten keine Leitungskonflikte auf. Auf der anderen Seite hat das Netz eine geringe Ausfallsicherheit. Wenn nämlich eine Störung in der

2 Eigene Darstellung in Anlehnung an Haaß (1997), S. 526. 
Zentrale auftritt, dann kann die Funktionsfähigkeit des gesamten Netzes darunter leiden. Eine sternförmige Netzstruktur wächst so lange, bis sie an ihre Kapazitätsgrenze stößt. Ab einer gewissen Anschlußzahl ist die Zentrale uberlastet. Sie wird zu einem Engpaß. Bei Kommunikationsnetzen äußert sich das durch ein Besetztzeichen bei den Grunddiensten wie z. B. bei einem Telefonanruf bzw. durch Warteschlangen bei Mehrwertdiensten wie bei Rufnummernauskünften. In einem solchen Zustand erhöht jeder weitere Nutzer die Wartezeiten der anderen. Es treten folglich negative Netzwerkexternalitäten auf. ${ }^{3}$ Wann und ob es dazu kommt, hängt vom verwendeten Material und der eingesetzten Technik ab. Bei herkömmlicher Durchleitung durch ein Kupfernetz mag eine Kapazitätsgrenze schnell erreicht sein. Mit neuen technologischen Errungenschaften wie der x-DSL Technologie ${ }^{4}$ oder bei Einsatz eines anderen Materials wie beispielsweise Glasfaser liegt die Kapazitätsgrenze weit von dem bisherigen Nutzungsniveau entfernt.

Das Fernnetz umfaßt gemäß der Abbildung 2.1-1 alle Vermittlungseinrichtungen außerhalb des Ortsnetzes, d.h. die Knotenvermittlungsstellen (KVSt), die Regionalvermittlungsstellen (RVSt) und die Weitverkehrsvermittlungsstellen (WVSt) sowie die dazugehörigen Leitungen und Querleitungen. Die Vermittlungsstellen sind wiederum hierarchisch aufgebaut. Allerdings muß eine Telefonverbindung in einem digitalen Netz nicht sklavisch die einzelnen Vermittlungsstellen durchlaufen. Die Vermittlungsstellen sind miteinander maschenartig verbunden, d.h. das Fernnetz weist eine maschenartige bzw. teilvermaschte Struktur auf.

Ein Maschennetz wird in der Regel auf Ebenen mit hohem Verkehrsaufkommen eingesetzt, also in der Regel auf höheren Netzebenen wie bei den WVSt. Bei einem Maschennetz wird zwischen einer Teil- und einer Vollvermaschung unterschieden. Bei einer Vollvermaschung ist jeder Knotenpunkt miteinander verbunden. Eine Teilvermaschung ist gegeben, wenn nicht alle Knotenpunkte miteinander vollständig vermascht sind. Je größer die Zahl der Knoten ist, desto höher ist die Zahl an Verbindungsleitungen. Aufgrund der starken Vermaschung und der Eigenschaft, daß die Verbindungsleitungen gleichrangig zueinander sind, ist das Netz äußerst robust. Es besteht eine sehr hohe Ausfallsicherheit. Ist ein Knoten oder eine Verbindungsleitung uberlastet oder ausgefallen, kann der Leitungsverkehr über andere Knoten und Leitungen ausweichen. Fällt ein Knoten aus, dann bleibt das Netz bis auf diesen einen Knoten funktionsfähig. Fällt eine Verbindungsleitung aus, bleiben alle Knoten in Funktion. Der Datenfluß zwischen allen anderen Knoten kann uber die noch funktionsfähigen Verbindungsleitungen laufen. Außerdem wird das Risiko eines Datenstaus minimiert, da aufgrund der hohen Vermaschungsdichte ausreichende Kapazität geschaffen wurde. Ein Ma-

Zu dem Begriff Netzwerkexternalităten vgl. Kap. 4.

Vgl. dazu Kap. 2.1.1.3 sowie Tabelle 2.1.-2. 
schennetz dürfte eigentlich mit zunehmender Größe nie an seine Kapazitätsgrenze stoßen, weil mit jedem weiteren Knotenpunkt sich auch die Vernetzung erhöht.

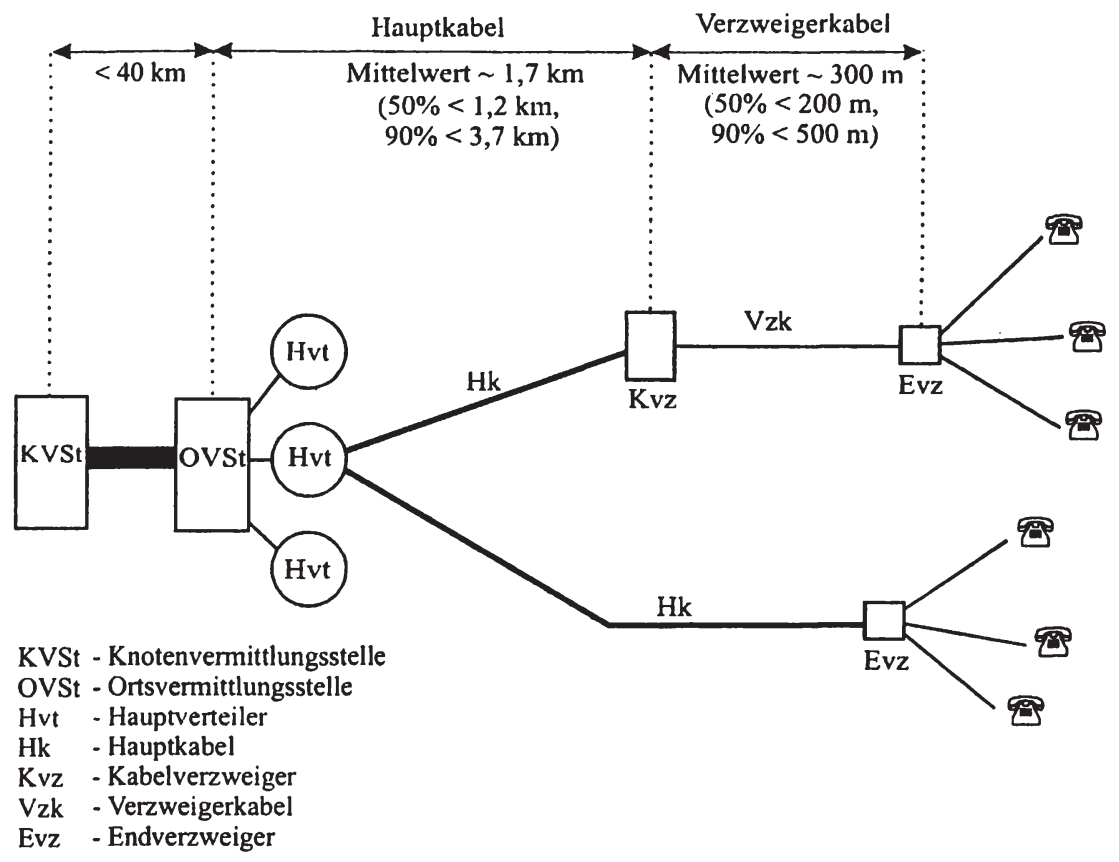

\section{Abbildung 2.1-2: Architektur eines Ortsnetzes mit Kupferdoppeladern ${ }^{5}$}

In der Abbildung 2.1-1 sind die möglichen Verbindungen zwischen fremden WVSt-Bereichen aufgezeigt. Der Anruf eines Teilnehmers wird in digitale Signale umgewandelt. Diese werden im Ortsnetz zu einer OVSt geschickt. Bei einem Gespräch im Nahbereich $(\leq 50 \mathrm{~km})$ wird der Telefondienst über KVSt und RVSt an die gerichtete OVSt weitergeleitet und von da aus an den gewünschten Teilnehmer übermittelt. Handelt es sich um ein Ferngespräch, werden die Informationen über eine KVSt direkt in den Weitverkehr vermittelt. Eine WVSt ist zuständig für den Weitverkehr zwischen fremden WVSt, sofern keine Querverbindungen zwischen KVSt und WVSt oder einer anderen KVSt vorhanden ist. Sie sind untereinander vollständig vermascht. Daraus folgt, daß, wenn der Direktweg zwischen zwei WVSts aus technischen Gründen ausfällt oder überlastet ist, ein

s In Anlehnung an Schmoll/Wist (1997), S. 2. 
Transit über eine dritte WVSt erfolgen kann. Außerdem gibt es noch die Möglichkeit der Querverbindungsleitungen (gestrichelte Linien) zwischen unterschiedlichen KVSts und zwischen WVSts und KVSts. Dank der vollständigen Vermaschung der WVSts und der Existenz der Querverbindungsleitungen muß die hierarchische Netzstruktur nicht zwangsweise vollzogen werden.

Die Netzinfrastruktur des Ortsnetzes erfordert eine detaillierte Betrachtung. In der Abbildung 2.1-2 wird die Architektur eines klassischen sternförmigen Ortsnetzes dargestellt. Dort liegt furr das gesamte Anschlußnetz eine Sternstruktur vor, die alle Teilnehmer verkabelt. Die Verlegung der Hauptkabel (Hk) und Verzweigerkabel (Vzk) von den Hauptverteilern (Hvt) bis hin zu den Endverzweigern (Evz) weisen dagegen eher eine Baumstruktur ${ }^{6}$ auf. Der zu vermittelnde Dienst wird schließlich von der OVSt über den HVT über Kvz und Evz an den gewünschten Teilnehmeranschluß weitergeleitet.

Das Telekommunikationsnetz läßt sich in verschiedene Ebenen unterteilen, in die sich die verschiedenen Funktionen eines Netzes, nämlich die physische Verbindung, die Übertragung und die Vermittlung, einordnen lassen.

\subsubsection{DIE PHYSISCHE EBENE}

Auf der physischen Ebene werden das Kupfer- und das Koaxialkabel bei der Übertragung elektrischer Signale und das Glasfaserkabel bei der Lichtwellenübertragung (LWL) als Übertragungsmedien im Festnetz am häufigsten verwendet. In der folgenden Tabelle 2.1-1 werden diese drei Übertragungsmedien anhand zentraler Merkmale wie der Übertragungsart und der Übertragungsrate, der Störempfindlichkeit und der Verlegbarkeit der Medien, der anfallenden Kosten, des verwendeten Materials sowie technischer Merkmale wie des Kabelaufbaus, der Übertragungstechniken und der technischen Verwendungsmöglichkeiten der einzelnen Übertragungsmedien kurz dargestellt. Aufgrund der tabellarischen Darstellung sind die genannten Übertragungsmedien für den Leser leichter vergleichbar.

Bei einer Baumstruktur sind die Teilnehmeranschlüsse an einem nicht vermaschten, baumformigen Netz angeschlossen. Vgl. Jung/Warnecke (1998), S. 91. 


\begin{tabular}{|c|c|c|c|}
\hline $\begin{array}{l}\text { Medium } \\
\text { Merkmal }\end{array}$ & Kupferkabel & Basisband & $\begin{array}{l}\text { Lichtwellenleiter / } \\
\text { Glasfaser }\end{array}$ \\
\hline $\begin{array}{l}\text { Übertragungs- } \\
\text { art / } \\
\text { Betriebsart }\end{array}$ & $\begin{array}{l}\text { analog / digital } \\
\text { uni- / bidirektional }\end{array}$ & $\begin{array}{|lr|}\text { digital } & \text { analog / digital } \\
\text { bidirektional } & \text { unidirektional }\end{array}$ & unidirektional \\
\hline $\begin{array}{l}\text { Übertragungs- }^{\text {rate }^{8}} \\
\end{array}$ & $16 \mathrm{Mbit} / \mathrm{s}$ & $\leq 600 \mathrm{Mbit} / \mathrm{s}$ & $\leq 10 \mathrm{Gbit} / \mathrm{s}$ \\
\hline $\begin{array}{l}\text { Störempfind- } \\
\text { lichkeit }\end{array}$ & $\begin{array}{l}\text { größer im Verhältnis zum Ko- } \\
\text { axialband und zum LWL }\end{array}$ & $\begin{array}{l}\text { gering } \\
\text { Isoliermaterialien halten Ableitungsver- } \\
\text { luste selbst bei hohen Frequenzen gerin- } \\
\text { ger als bei einfachem Kupferkabel. }\end{array}$ & sehr gering \\
\hline Verlegbarkeit & sehr gut & gut & schwierig $^{9}$ \\
\hline Kosten & geringe Materialkosten & $\begin{array}{c}\text { höhere Materialkosten als beim Kupfer- } \\
\text { kabel }\end{array}$ & $\begin{array}{l}\text { geringe Materialkosten (ge- } \\
\text { schmolzener Quarzsand) } \\
\text { hohe Anschlußkosten }^{10}\end{array}$ \\
\hline Material & $\begin{array}{l}\text { Ein Kabel besteht aus } 2-4000 \\
\text { Kupferleitungen. Isolierung: } \mathrm{Pa}- \\
\text { pier oder Kunststoff. Mantel: } \\
\text { Kunststoff, Blei oder Stahl. }\end{array}$ & $\begin{array}{l}\text { Kupfer ist das Leitmedium. Der Außen- } \\
\text { leiter besteht aus Keramik oder Poly- } \\
\text { äthylen. }\end{array}$ & $\begin{array}{l}\text { Kern und Mantel sind aus op- } \\
\text { tisch transparentem Material er- } \\
\text { stellt, z. B. Quarzglas. }\end{array}$ \\
\hline
\end{tabular}

7 Die Betriebsart bezieht sich auf die Art der Benutzung der Verbindungsleitungen. Wird eine Verbindungsleitung wechselseitig benutzt bzw. ist sie bidirektional gerichtet, dann existiert zwischen zwei Knoten nur eine Leitung, die in beide Richtungen den Verkehr ermöglicht. Im Fall einer gerichteten bzw. unidirektionalen Verbindungsleitung kann der Verkehr dementsprechend nur in eine Richtung erfolgen. Bei $n$ Knoten ergeben sich $n(n-1)$ Verbindungsleitungen bei gerichtetem Verkehr. Wird jede Leitung wechselseitig betrieben, dann gibt es bei $n$ Knoten $n(n-1) / 2$ Verbindungswege.

$8 \quad 1 \mathrm{kbit} / \mathrm{s}=1024 \mathrm{bit} / \mathrm{s}=2^{10} \mathrm{Bit} ; 1 \mathrm{Mbit} / \mathrm{s}=1048576 \mathrm{bit} / \mathrm{s}=2^{10} \mathrm{kbit} / \mathrm{s} ; 1 \mathrm{Gbit} / \mathrm{s}=1048576 * 2^{10} \mathrm{kbit} / \mathrm{s}=2^{10} \mathrm{Mbit} / \mathrm{s}$

9 Bei der Konstruktion muß berücksichtigt werden, daß die Fasern nicht gekrümmt und nicht brechen dürfen. Die Übertragungseigenschaften müssen bei allen mechanischen und thermischen Belastungen während der Kabelverlegung und im Betrieb konstant gehalten werden. D.h. die Verlegbarkeit von Glasfaserkabel kann als schwieriger eingestuft werden als beim Kupferkabel oder beim Koaxialband.

10 Deswegen lohnt sich ein Ausbau dieses Netzes hauptsächlich nur in dicht besiedelten Ballungsräumen. 


\begin{tabular}{|c|c|c|c|}
\hline \begin{tabular}{|l} 
Medium \\
Merkmal
\end{tabular} & Kupferkabel & $\begin{array}{ll}\text { Kasisband } & \text { Koaxial- }\end{array}$ & $\begin{array}{l}\text { Lichtwellenleiter / } \\
\text { Glasfaser }\end{array}$ \\
\hline Kabelaufbau & $\begin{array}{l}\text { Symmetrischer Kabelaufbau: d.h. } \\
\text { je zwei voneinander isolierte } \\
\text { Adern werden als Doppeladern zu- } \\
\text { sammengefaßt, um interne Störein- } \\
\text { flüsse zu reduzieren. Man spricht } \\
\text { auch von Twisted-pair- Kabeln. } \\
\end{array}$ & $\begin{array}{l}\text { Zweileitersystem; d.h. ein Außenleiter } \\
\text { umschließt einen Innenleiter. }\end{array}$ & $\begin{array}{l}\text { Der Kabelaufbau besteht aus } \\
\text { einem Kern, einem Mantel und } \\
\text { einer Beschichtung. Im Kern } \\
\text { findet die Lichtwellenführung } \\
\text { statt. }\end{array}$ \\
\hline $\begin{array}{l}\text { Übertragungs- } \\
\text { technik }\end{array}$ & $\begin{array}{l}\text { Die Übertragung erfolgt anhand } \\
\text { von elektrischen Signalen. }\end{array}$ & $\begin{array}{l}\text { Das zu übertragende Signal wird an der } \\
\text { äußersten Oberfläche der Innenseite des } \\
\text { Außenleiters transportiert. Das Signal } \\
\text { dringt nicht bis zur Außenfläche des Au- } \\
\text { Benleiters. Deswegen können hochfre- } \\
\text { quente Störungen von außen das Nutzsi- } \\
\text { gnal nicht beeinflussen. }\end{array}$ & $\begin{array}{l}\text { Die Übertragung erfolgt anhand } \\
\text { von optischen Signalen. Die } \\
\text { Übertragungsgenauigkeit der } \\
\text { Signale ist im Vergleich zu den } \\
\text { elektrischen Signalen viel hö- } \\
\text { her. Die optischen Signale müs- } \\
\text { sen erst nach einer langen Ent- } \\
\text { fernung verstärkt werden. }\end{array}$ \\
\hline Verwendung & $\begin{array}{l}\text { Kupferkabel finden als ältestes } \\
\text { Übertragungsmedium im Bereich } \\
\text { des natürlichen Sprachbandes und } \\
\text { für Trägerfrequenz- und Digitalsy- } \\
\text { steme in den unteren Netzebenen } \\
\text { Verwendung, also nur im Ortsnetz. }\end{array}$ & $\begin{array}{l}\text { Das Basisband-K. wird i.d.R. für digitale } \\
\text { Übertragung genutzt. Man findet es in } \\
\text { LANs, bei Fernverbindungen im Tele- } \\
\text { fonnetz und bei Breitband-Anschlußlei- } \\
\text { tungen im Ortsnetz. } \\
\text { Das Breitband-K. ist die erste Generation } \\
\text { der Kabelfernsehnetze und wird bei der } \\
\text { analogen Übertragung von Fernsehen } \\
\text { und Hörfunk verwendet. }\end{array}$ & $\begin{array}{l}\text { Die Verwendung von Glasfaser } \\
\text { im Zusammenhang mit der } \\
\text { Lichtwellentechnik eignet sich } \\
\text { besonders für hochvolumige } \\
\text { Netzebenen in der Breitband- } \\
\text { übertragung. }\end{array}$ \\
\hline
\end{tabular}

Tabelle 2.1-1: Merkmale einzelner Übertragungsmedien 


\subsubsection{DIE LOGISCHE EBENE}

Die logische Netzebene bezieht sich auf die Übertragung und die Vermittlung von Sprache bzw. Dateneinheiten. Dabei kann eine analoge oder digitale Übertragung vorgenommen werden. Die Vermittlung kann in Form von Leitungs- oder Paketvermittlung erfolgen.

\section{Übertragung}

Analoge Übertragung von Sprache bzw. Daten funktioniert über wert- und zeitkontinuierliche elektrische Schwingungen. Bei der digitalen Übertragung handelt es sich um wert- und zeitdiskrete Signale. Das Signal wird als eine Zeichenfolge binärer Informationen kodiert und in diesem kodierten Zustand übertragen.

\section{Vermittlung}

Vermittlungsnetze werden in der Regel für Telekommunikationsdienste wie Telefon, Datenfernübertragung und Datenverarbeitung eingesetzt. Thre Aufgabe ist es, eine Vermittlung zwischen mindestens zwei Endgeräten von Teilnehmern herzustellen. Beim deutschen kabelgebundenen Fernsprechnetz handelt es sich um ein schmalbandiges oder breitbandiges Vermittlungsnetz. "

Es liegt weitestgehend eine digitale Übertragungstechnik vor. Die Digitalisierung des analogen Fernsprechnetzes vollzog sich ab 1975. Mit Hilfe von PCMÜbertragungssystemen ${ }^{12}$ zwischen den Orts- und Knotenvermittlungsstellen wurde eine Mehrfachausnutzung von Kabelstrecken ermöglicht. Die Vermittlungstechnik stützte sich auf Analog-Digital-Wandler, die die analogen Fernsprechsignale an den Ein- und Ausgängen der Vermittlungsstellen verarbeiten. Die digitale Übertragungstechnik hat schließlich nach und nach die analogen Systeme verdrängt, weil sie von der Übertragungsqualität und von der Übertragungsgeschwindigkeit deutlich überlegen ist. ${ }^{13}$

Es folgt als eine technisch weiterführende Entwicklungsstufe die digitale Vermittlungstechnik, d.h. im Orts- und Ferndienst werden digitale Vermittlungsstellen eingesetzt. Die digitale Vermittlungstechnik macht es möglich, daß ver-

"Ein schmalbandiges Netz umfaßt Frequenzbandbreiten bis zu $3100 \mathrm{~Hz}$. Das Breitband dagegen umfaßt die Frequenzbandbreiten, die über $3100 \mathrm{~Hz}$ liegen.

12 PCM - Puls Code Modulation. Die PCM 30 Übertragungseinrichtung ist eine Multiplextechnik. Das Multiplexverfahren ist eine Methode, die mehrere Informationskanäle mit niedriger Übertragungsgeschwindigkeit in einen Kanal mit höherer Geschwindigkeit zusammenfaßt. PCM 30 codiert und decodiert analoge Signale in digitale. Sind die Zugangssignale bereits digital, dann müssen sie nicht mehr umgewandelt werden. Dann ist folglich die PCMCodierung nicht mehr notwendig. Vgl. Haaß (1997), S. 99 ff., S. 528. 
schiedene Informationstypen (Sprach-, Text-, Daten- und Bildinformationen) über ein Netz ausgetauscht werden können. Es sind keine unterschiedlichen Netze mit unterschiedlichen Anforderungen mehr notwendig. Ein herkömmlicher Teilnehmeranschluß verfügt mit Hilfe der digitalen Vermittlungstechnik über eine Übertragungskapazität von $56 \mathrm{kbit} / \mathrm{s}^{14}$ Das sogenannte Schmalband-Integrated Services Digital Network (S-ISDN) ist ein diensteintegrierendes digitales Fernmeldenetz. Es wird als technische Weiterentwicklung der bisherigen Fernmeldenetze gesehen. Es wächst aus dem analogen System heraus und soll es nach und nach vollstăndig ersetzen. Ziel ist folglich, alle Nutzergruppen, betriebliche wie private, anzuschließen. Die Teilnehmeranschlüsse verfügen mit ISDN über eine Übertragungskapazität von $144 \mathrm{kbit} / \mathrm{s}$. Neben der Zusammenfassung aller Übermittlungsdienste in ein universelles Nachrichtennetz ${ }^{15}$ leistet das ISDN einen schnelleren Verbindungsaufbau durch die digitale Signalisierung. Der Vorteil an ISDN ist, daß es über herkömmliche Kupferdoppeladern betrieben werden kann.

Die International Telecommunications Union (ITU) hat sogenannte Empfehlungen über internationale Standardisierungen bezüglich des ISDN ausgesprochen. Sie sollen eine einheitliche Grundlage für die Telekommunikationstechnik verschiedener Nationen schaffen. Die Empfehlungen besitzen keinen bindenden Charakter, werden von den einzelnen Nationen aber weitgehend eingehalten. ${ }^{16}$

\subsubsection{DIE TECHNISCHE WEITERENTWICKLUNG}

In bezug auf die technische Evolution des Fernsprechnetzes wird auf die digitale Vermittlungstechnik das Breitband-ISDN (B-ISDN) mit der ATM-Technik ${ }^{17}$ folgen. Es steht nicht in Konkurrenz zu dem gegenwärtigen Schmalband-ISDN, sondern soll es vielmehr um den breitbandigen Anteil ergänzen. Es vereint als erstes Netz Verteildienste und Vermittlungsdienste in einem einheitlichen Netz. Auf diese Weise ermöglicht es das Zusammenwachsen der TIME-Märkte. ${ }^{18}$ Es ermöglicht

\footnotetext{
14 Die Netzkapazităt ergibt sich aus der Höhe an Information, die pro Zeiteinheit vermittelt werden kann.

is Für den Konsumenten hat es zur Folge, daß er alle Telekommunikationsdienste über einen Netzanschluß gleichzeitig in Anspruch nehmen kann.

16 Weitere Übertragungsnetze neben dem ISDN sind das herkömmliche öffentliche Telefonnetz, das leitungsvermittelnde Datennetz (Datex- $L$ ), das paketvermittelnde Datennetz (Datex-P), die Rechnernetze und die D- und E- Mobilfunknetze. Datex-L und Datex-P sind Dienste, die im integrierten Text- und Datennetz (IDN) angeboten werden. Das IDN existiert schon seit 1976 und ist ein digitales Datennetz, das auf die Übermittlung nicht sprachlicher Daten ausgelegt ist. Vgl. hierzu Kap. 3.4.1.3 zur Internet-Telefonie .

17 ATM - Asynchronous Transfer Mode. Die ATM-Technik ist nach Empfehlung der ITU das Übermittlungsprinzip des B-ISDN. Vgl. HaaB (1997), S. 438. Eine ausführlichere Darstellung der Funktionsweise von ATM vgl. im Anhang A.1, Fundneider (1994), S. 29 ff.

TIME - Telecommunications, Information, Media, Entertainment, Electronics
} 
den Benutzern eine standortübergreifende Nutzung von Multimedia-Diensten, Verteilung von Ton- und Bildinformationen', verbindungsloser sowie verbindungsorientierter Breitbandübermittlungstechniken, Video on Demand, Koppelung zwischen entfernten ,Local Area Networks' (LANs) und ,Metropolitan Area Networks' (MANs) mit sehr hohen Geschwindigkeiten sowie die Integration vorhandener Schmalbanddienste. ${ }^{20}$

Das B-ISDN ${ }^{21}$ hat ebenso wie das S-ISDN den Vorteil, daß es sowohl über Kupferdoppeladern, Koaxialkabel als auch über Glasfaserkabel laufen kann, so daß die bisherigen Anschlüsse weiter genutzt werden können. Es funktioniert ebenso gut mit kabelungebundenen Übertragungsmedien, z. B. über Mobilfunk und Satellit.

Mit der ,Digital Subscriber Line‘ Technologie, kurz DSL, können herkömmliche Kupferkabelanschlüsse für die Breitbandkommunikation im Rahmen des B-ISDN aufgerüstet werden, so daß die letzte Meile bezüglich der Datenübertragungsgeschwindigkeit keinen Engpaß mehr darstellt. Allerdings hängen die technisch zu erzielenden Übertragungsraten von der Distanz des Kunden zur TVSt ab. Für die Verfügbarkeit eines DSL-Anschlusses ist die Aufrüstung sowohl der OVSt als auch des Teilnehmeranschlusses mit jeweils einem Splitter und mit einem DSLModem notwendig.

Es gibt mehrere Varianten des DSL-Verfahrens, die in der Tabelle 2.1-2 aufgeführt sind. Sie unterscheiden sich in den Übertragungsraten und in der Reichweite. Generell gilt, je höher die Übertragungsrate ist, desto kürzer ist die Reichweite. High Bit Rate Digital Subscriber Line (HDSL) ermöglicht beispielsweise eine bidirektionale Übertragung von $2 \mathrm{Mbit} / \mathrm{s}$ über eine Reichweite von ca. $3 \mathrm{~km}$. Es handelt sich allerdings hierbei um eine reine Datentechnik und benötigt zwei Doppeladern, so daß analoge wie digitale Telefonie damit nicht möglich sind. Die Symmetric oder Single Line Digital Subscriber Line -SDSL - Technik setzt nur auf einer Kupferdoppelader auf. Thre Übertragungsrate beträgt 2,3 Mbit/s. Daneben sind parallel $64 \mathrm{kbit} / \mathrm{s}$ Datenkanäle eingebunden, die für die Bereitstellung von Telefon- oder ISDN -Kanälen genutzt werden können. Asymmetrical Digital

19 Zum Beispiel für die Übertragung hochaufgelöster medizinischer Aufnahmen und medizinischen Informationen oder für CAD / CAM / CIM Daten.

20 Vgl. Haaß (1997), S. 435. LAN und MAN sind Netzwerkstrukturen des Internets, die in Kap. 3.4.1.3 etwas näher beschrieben werden.

21 Die Aufbauphase der Breitbandkommunikation begann Mitte 1994 mit einem überregionalen Pilotnetz der DTAG mit 3 ATM-Vermittlungsknoten. Inzwischen ist dieses Netz deutschlandweit ausgebaut und verbindet die großen Wirtschaftszentren miteinander. 1996 wurden die Knoten $\mathrm{zu}$ voll funktionalen Vermittlungsstellen aufgerüstet, so daß auch Wählverbindungen unterstützt werden können. 
Subscriber Line -ADSL ${ }^{22}$ ist bislang die am meisten verwendete Technik. Es ist ein unsymmetrisches Verfahren, bei dem bis zu $8 \mathrm{Mbit} / \mathrm{s}$ uber eine Reichweite von $5 \mathrm{~km}$ in Richtung Kunde (downstream) und $640 \mathrm{kbit} / \mathrm{s}$ in Richtung Netz (upstream) transportiert werden können. ${ }^{23}$ Mit Very High Bit Rate Digital Subscriber Line -VDSL- lassen sich Übermittlungsraten bis zu $52 \mathrm{Mbit} / \mathrm{s}$ downstream und 1,6 Mbit/s upstream sowie eine symmetrische Aufteilung der Kapazitäten von $26 \mathrm{Mbit} / \mathrm{s}$ in beide Richtungen tuber eine Kupferdoppelader realisieren. Aufgrund der hohen Übertragungsgeschwindigkeiten haben VDSL Übertragungen nur eine Reichweite auf 0,3 bis zu 1,5 km und können dementsprechend nur im letzten Teil des Kabelnetzes ( Verzweigernetz ) eingesetzt werden. ${ }^{24}$

\begin{tabular}{|l|c|c|c|}
\hline \multicolumn{1}{|c|}{ Variante } & Übertragungsrate & Reichweite & Telefonie \\
\hline Analog & $56 \mathrm{kbit} / \mathrm{s}$ & unbegrenzt & ja \\
\hline ISDN & $144 \mathrm{kbit} / \mathrm{s}$ & unbegrenzt & ja \\
\hline HDSL & $2 \mathrm{Mbit} / \mathrm{s}$ & $3-4 \mathrm{~km}$ & nein \\
\hline SDSL & $2 \mathrm{Mbit} / \mathrm{s}$ & $2,5 \mathrm{~km}$ & ja \\
\hline ADSL (upstram) & $640 \mathrm{kbit} / \mathrm{s}$ & $5 \mathrm{~km}$ & ja \\
\hline ADSL (downstream) & $8 \mathrm{Mbit} / \mathrm{s}$ & $5 \mathrm{~km}$ & ja \\
\hline VDSL & $52 \mathrm{Mbit} / \mathrm{s}$ & $1,5 \mathrm{~km}$ & ja \\
\hline
\end{tabular}

Tabelle 2.1-2: X-DSL- Übertragungsvarianten im Vergleich ${ }^{25}$

95\% aller Leitungen im urbanen Bereich haben eine durchschnittliche Länge von $1,9 \mathrm{~km}$ bis hin zum HVT. ${ }^{26}$ D.h. der größte Teil des Teilnehmeranschlußbereichs in Deutschland kann fur ADSL und HDSL erschlossen werden. ${ }^{27}$

22 Dieses Verfahren wird derzeit von der DTAG als T-DSL angeboten und haben bereits eine marktbeherrschende Stellung. Vgl. hierzu Kap. 6.3.1, Huber (1999), S. 30 ff., Rathgeb (1998), S. 19 ff., Rathgeb/Wallmeier (1997).

23 DSL ist aus diesem Grund interessant für ,Video on Demand' Dienste.

24 Die durchschnittliche Entfernung von einer Vermittlungsstelle bis zum EndanschluB entspricht 2-4 km. Vgl. Siegmund (1999), S. 216 f. und Abbildung 2.1-2.

25 In Anlehnung an RegTP (2001a), S. 8.

26 Vgl. RegTP (2001a), S.7.

27 Für die restlichen $5 \%$ wăre die Anwendung von DSL- Verfahren unverhăltnismäßig kostspielig, so daß eine $100 \%$ ige Abdeckung unwahrscheinlich ist. 


\subsubsection{Die Diensteebene}

Nachdem die Infrastrukturebene in ihren technischen Details erläutert worden ist, soll nun auf die Diensteebene eingegangen werden. Dabei läßt sich die Diensteebene am besten an der Abbildung 2.1-3 darlegen.

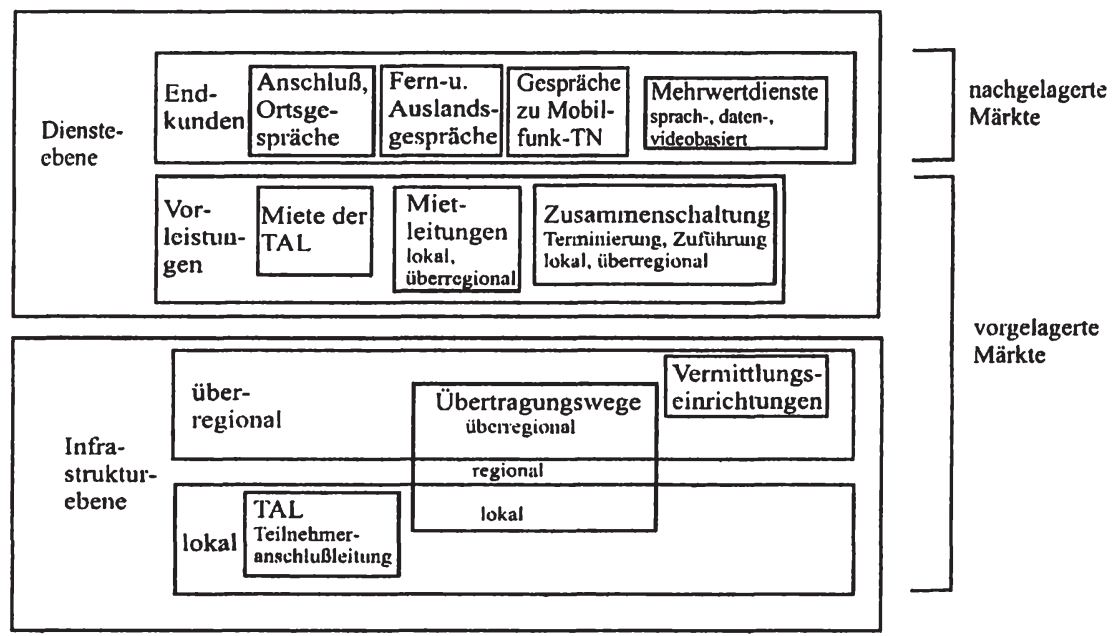

\section{Abbildung 2.1-3: Sektorstruktur in der Telekommunikation ${ }^{28}$}

Abbildung 2.1-3 zeigt die sektorale Struktur der deutschen Telekommunikation, an der die Trennung zwischen Infrastruktur- und Diensteebene nachvollzogen wird. Die Infrastrukturebene ist in eine lokale und eine überregionale unterschieden worden. Auf der lokalen Ebene werden neben der Teilnehmeranschlußleitung örtliche Übertragungswege bereitgestellt. Auf der überregionalen Ebene befinden sich neben den Übertragungswegen die Vermittlungseinrichtungen.

Auf der Diensteebene wurde eine Trennung in Vorleistungs- und Endkundenmärkte vollzogen. Vorleistungen sind Leistungen im vorgelagerten Markt, die der etablierte Netzbetreiber den Marktneulingen bei der Möglichkeit von Dienstewettbewerb $^{29}$ entgeltlich zur Verfügung stellt, zum Beispiel die Vermietung von Teilnehmeranschlußleitungen, lokale und überregionale Leitungen und die $\mathrm{Zu}-$ sammenschaltung für den Übergang zu anderen Netzbereichen. Endkundendienste

28 In Anlehung an Kruse (2000), S. 404.

29 Eine Erläuterung des Begriffs Dienstewettbewerb erfolgt in Kap. 3.1.2. 
dagegen sind zum Beispiel die Bereitstellung eines Teilnehmeranschlusses (ana$\log$, digital, ISDN oder $\mathrm{x}$-DSL) für den Konsumenten und die Vermittlung von Telefongesprächen (Orts-, Fern- und Auslandsgespräche) sowie von Mehrwertdiensten (Sprach-, integrierte und Datenmehrwertdienste, Multimediadienste). ${ }^{30}$

\subsection{Einführung In DIE WeTtbeWerbSPOltische Problem- STELLUNG FÜR DEN ORTS- BZW. ANSCHLUBBEREICH}

In der folgenden Arbeit soll das Augenmerk auf den Nahverkehrbereich gelegt werden. Dieser zeichnet sich dadurch aus, daß sich dort seit der Liberalisierung immer noch kein Wettbewerb eingestellt hat. Dabei ist die Strecke von der OVSt bis hin zum Teilnehmeranschluß der Teil, der wettbewerbspolitisch besonders brisant ist. ${ }^{31} \mathrm{Ihr}$ werden Eigenschaften eines resistenten Monopols unterstellt. Das bedeutet, daß es zu keinem Markteintritt kommt, selbst wenn das Monopolunternehmen produktiv wie allokativ vollkommen ineffizient ${ }^{32}$ arbeitet und $\mathrm{zu}$ hohe Preise bei zu hohen Kosten verlangt. Eine Monopolstellung ist unter anderem auf die Subadditivität zurlickzuführen. ${ }^{33}$

Im Zuge der digitalen Vermittlungs- und Übertragungstechnik hat sich allerdings die Topologie von lokalen Telekommunikationsnetzen grundlegend geändert. Aus der Digitalisierung der Übertragungs- und Vermittlungstechnik ergibt sich, daß einzelne OVStn von abgesetzten Konzentratoren ersetzt werden können. Konzentratoren bilden den Übergang zwischen Anschlußleitung und gemeinsam genutzten Komponenten. Sie sammeln einzelne Daten und konzentrieren Vermittlungsfunktionen und leiten sie zusammengefaßt an eine hierarchisch über ihnen stehende VSt. Die jeweiligen OVStn erhalten auf diese Weise einen größeren Einzugsbereich, der nicht mehr deckungsgleich mit den politischen Gemeindegrenzen sein muß. Folglich reduziert sich die Zahl der OVStn. Daraus ergeben sich zwei hervorzuhebende Trends:

Erstens fuhrt die Reduzierung der Zahl der OVStn zu einer Veränderung der Topologie des Ortsnetzes. Die Entfernung der Teilnehmeranschlußleitungen bis hin zu einer OVSt können sich von durchschnittlich $2 \mathrm{~km}$ auf bis zu $50 \mathrm{~km}$ erhöhen. Daraus folgt, daß die Bezeichnung Ortsnetz einen größeren geographischen Be-

30 Eine ausfuihrliche Darstellung der einzelnen Vorleistungen sowie der Endkundendienste erfolgt im Rahmen einer Marktabgrenzung in Kap. 3.3.

31 Vgl. hierzu Abbildung 2.1-2.

32 Zu den Begriffen allokative und produktive Effizienz vgl. Kap. 5.1.

33 Vgl. dazu Kap. 2.3. 
reich abdeckt. ${ }^{34}$ Vor der Digitalisierung erfüllte das Ortsnetz die Eigenschaften eines natürlichen Monopols. Die Netzstruktur, die inzwischen unter einem Ortsnetz zusammengefaßt ist, erfullt diese nicht mehr, sondern nur noch ein Teil aus dem Ortsnetz. Für die folgende Arbeit ist eine genaue Differenzierung der Netzteile unbedingt erforderlich, denen nach wie vor ein Bottleneck-Charakter nachgesagt werden kann und die aufgrund von Kostensubadditivität natürlichen Monopolen entsprechen. Das European Telecommunications Standard Institute (ETSI) benutzt deswegen nicht mehr den Begriff Ortsnetz, sondern die Unterteilung in $\mathrm{Zu}$ gangsnetz (access network), das die gemeinsam genutzten Einrichtungen bezeichnet, und Verteilnetz (distribitution network), das die exklusiven Anschlußleitungen, die von einzelnen Teilnehmern genutzt werden, umfaßt. ${ }^{35}$ Der Begriff des Verteilnetzes ist aufgrund der Entwicklungen der Anschlußtechnologien sehr eng gefaßt. Wenn das Ziel verfolgt wird, diejenigen Bereiche zu spezifizieren, denen am ehesten die Eigenschaften eines Engpaßfaktors zukommen, ist die Unterteilung in Zugangsnetz und Verteilnetz nicht sinnvoll, da in beiden Netzkategorien Eigenschaften eines Engpaßfaktors vorliegen. Zieladäquate Begriffe könnten die Letzte Meile oder der Teilnehmeranschlußbereich sein, die die Infrastruktur des wettbewerbspolitisch besonders kritischen Netzteils beschreiben. Sie umfassen unabhängig von gemeinsam oder individuell genutzten Einrichtungen die letzten zwei Kilometer bis hin zum Teilnehmeranschluß.

Zweitens schafft die Digitalisierung auf Vermittlungs- und Übertragungsebene die Voraussetzung für eine Übertragung von größeren Nutzbandbreiten bis hin zum Teilnehmer. Dies ermöglicht zum einen einen qualitativen Sprung für die bisher angebotenen Telekommunikationsdienste. Zum anderen verhilft es zu einer Bereitstellung völlig neuartiger Produkte, sogenannter Breitbanddienste. ${ }^{36}$

In der Liberalisierungsphase entstanden ordnungspolitische Schwierigkeiten aufgrund der vertikalen Integration zwischen Netz- und Diensteebene. ${ }^{37}$ Vertikale strukturelle Separierung war rechtlich und politisch nicht durchsetzbar. Aus diesem Grund ergab sich zunächst auch weitestgehend ein ,One-Way-Access'. Das bedeutet, daß der etablierte Netzbetreiber - der Incumbent - den vorgelagerten Markt monopolisiert, auf den andere Anbieter im nachgelagerten Markt sowie auch Anbieter von Netzinfrastruktur zurückgreifen müssen. Dabei kann der nachgelagerte Markt durchaus schon von Wettbewerb gekennzeichnet sein. Dagegen ist das Monopol im vorgelagerten Markt nicht von den Anbietern im Endpro-

\footnotetext{
34 Vgl. Schmoll/Wist (1997), S. 1. Die Glasfaserverlegung in das Ortsnetz hinein verändert zudem die Netzinfrastruktur.

35 ETSI unterstellt eine Netzdigitalisierung bis in die Anschlußebene.

36 Vgl. die Konsequenzen für das Bestehen eines natürlichen Monopols daraus in Kap. 2.3. Eine konkrete Marktabgrenzung der vielfältigen Dienste erfolgt in Kap. 3.3.

37 Vgl. Kruse (1997).
} 
duktmarkt abhängig. Der Incumbent verkauft als vertikal integrierter Monopolist Bottleneck-Dienste an den Newcomer. Insbesondere der Teilnehmeranschlußbereich stellt einen One-Way-Access dar. So bestand und besteht nach wie vor bei Einfuhrung von Wettbewerb auf der Diensteebene das Problem eines diskriminierungsfreien Zugangs zu den fraglichen Netzelementen für alle Konkurrenten. In Deutschland wurde versucht, dieses Problem mit Hilfe einer aufwendigen Bottleneck-Regulierung zu lösen. ${ }^{38}$

Ein ,Two-Way-Access' bezieht sich auf eine Situation, in der mehrere Netzwerkbetreiber existieren. ${ }^{39}$ Sie benötigen jeweils Zugang zum Netz des anderen Anbieters (Interconnection). Es gibt zwei Two-Way-Access Typen. Der eine Typ bezieht sich auf Netzwerke, die sich regional nicht überschneiden, wie beispielsweise bei internationalen Netzwerkbetreibern, die jeweils eine Nation mit Telekommunikationsdiensten versorgen (France Telecom und Deutsche Telekom). Ihre Netze stehen in einem komplementären Verhältnis zueinander. Der andere Typ umfaßt lokale Netzbetreiber, die bei infrastrukturbasiertem Wettbewerb um Kunden im Anschlußbereich konkurrieren und folglich in einem substitutiven Verhältnis zueinander stehen. In beiden Fällen ist eine Zusammenschaltung der Netze sowohl für beide Betreiber als auch für die Kunden von Vorteil, weil eine größere Zahl an Teilnehmern erreicht werden kann. ${ }^{40}$

\subsection{DIE TEILNEHMERANSCHLUBLEITUNG ALS NATÜRLICHES MONOPOL}

Die kabelgebundenen Teilnehmeranschlußleitungen auf der Infrastrukturebene des Telekommunikationsnetzes dienten bisher als Musterfall für ein naturliches Monopol in bezug auf eine zusätzliche Verkabelung im Teilnehmeranschlußbereich, weil es die zentralen Eigenschaften, nämlich das Bestehen potentieller Kostenvorteile bei monopolistischer Produktion (Kostensubadditivităt) erfüllt, die auf die Existenz von Skaleneffekten zurückzufuhren sind.

Erstens liegen erhebliche Größenvorteile in Form von horizontalen 'Economies of Scale' vor. Man spricht von dem sogenannten $2 / 3$ Effekt, d.h. die Oberfläche einer Leitung wächst ungefähr um 2/3 langsamer als das Volumen. Daraus folgt, daß

38 Zur gesetzlichen Regelung über die Gewährung von Netzzugängen vgl. Kap. 2.4 und Kap. 5.1, 5.2. Zur Bestimmung von Zugangsentgelten vgl. Kap. 5.4.

39 Armstrong (1996, 1998) und Laffont/Rey/Tirole (1997, 1998) sowie Laffont/Tirole (1994b, 1996, 2000) benutzen die Begriffe ,One-Way-Access' und ,Two-Way-Access'.

40 Zur gesetzlichen Regelung von Zusammenschaltung vgl. Kap. 2.4.3 sowie Kap. 4 zur ökonomischen Bewertung von Zusammenschaltung bei Netzeffektgütern. Zu möglichen Preisabsprachen zwischen den Anbietern vgl. Kap. 5.3. 
die durchschnittlichen Kosten des Aushebens eines Kabelschachtes, also die irreversiblen Fixkosten, pro Dateneinheit kleiner werden, wenn anstelle eines dünnen Kabels ein dickes verlegt wird, weil sich der Datendurchsatz bei einem dicken Kabel erhöht. Der Netzanbieter hat dementsprechend durch die Bündelung von Leitungen niedrigere Kosten als zwei miteinander im Wettbewerb stehende Anbieter, die ihre Kabel getrennt verlegen.

Zweitens existieren Dichtevorteile in einem regional abgegrenzten Gebiet. Je gröBer die Zahl der anzuschließenden Haushalte ist, desto geringer werden die infrastrukturellen Kosten pro Haushalt. Daraus folgt, daß die Stückkosten mit zunehmender Nachfragedichte fallen. Da Dichtevorteile in Ballungsgebieten in der Regel höher als in ländlichen Gebieten sind, kommen sie auch dort besonders zum Tragen. ${ }^{41}$

Drittens gibt es bei der Produktion verschiedener Güter, z.B. verschiedener Netzdienste, Verbundvorteile. Die gemeinsame Produktion ist zu geringeren Stückkosten möglich als die getrennte Produktion. ${ }^{42}$

Viertens liegen stochastische Durchmischungseffekte vor. ${ }^{43}$ D.h. ist die Zahl der Nachfrager besonders groß, dann verteilt sich bei stochastischer Nachfrage die Gesamtnachfrage gleichmäßiger über einen Zeitraum. Die benötigte Netzkapazität pro Teilnehmer sinkt mit steigender Anzahl an Nutzern. Folglich kann die Errichtung eines Netzwerkes mit einer geringeren Netzkapazität erzielt werden als die Errichtung von zwei Netzwerken bei gleicher Wahrscheinlichkeit einer Netzblockade $^{44}$. Je größer das Netz ist, desto geringer wird der Bedarf an Reservekapazitäten.

Die Nachfrage nach Telekommunikationsdiensten in den meisten Teilnehmeranschlußbereichen war bislang sowohl in der Peripherie als auch in vielen Ballungsregionen nicht ausreichend groß genug, um Größen-, Dichte- und Verbundvorteile

41 Die Versorgung mit Telekommunikationsdiensten in dünn besiedelten Regionen weist höhere Produktionskosten auf. Dichtevorteile treten dort im Verhältnis zu Ballungsregionen weniger stark auf.

42 Außerdem können noch vertikale Verbundvorteile auftreten. Sie entstehen bei vertikaler Produktion, d.h. ein Produkt ist Input-Faktor für das andere. Vertikale Verbundvorteile liegen vor, wenn es billiger ist, nachfolgende Produktionsstufen in einem Betrieb zu organisieren statt über Verträge zwischen ökonomisch selbständigen Betrieben. Das hieße in bezug auf Telekommunikationsdienste, den vor- und den nachgelagerten Markt in einer unternehmerischen Hand zu lassen. Der Erfolg der Call-by-Call Dienste im Zuge der Liberalisierung in Deutschland beweisen das Gegenteil, allerdings aufgrund von regulatorischen Eingriffen (Vgl. Kap. 2.2.4).

43 Vgl. Klodt, H.; u.a. (1995), S. 50.

44 Eine Netzblockade ist wahrscheinlich, wenn zu einem bestimmten Zeitpunkt so viele Leute die Netzleitungen beanspruchen, so daß für einen zusätzlichen Teilnehmer keine Kapazitäten mehr zur Verfügung stehen. 
sowie die stochastischen Durchmischungseffekte $\mathrm{zu}$ überwinden. Aus diesem Grund lag und liegt weitestgehend ein natürliches Monopol in den Anschlußbereichen vor. Der rasante technische Fortschritt hat allerdings Folgen fur die Marktabgrenzung und Marktstruktur auf der Letzten Meile, weil er zum einen die Kosten reduziert, zum anderen die Nachfrage stimuliert. Es steht in Frage, ob der lokale Netzbetreiber seine monopolistische Marktmacht in der langen Frist aufrecht erhalten wird. Für die weitere Analyse werden die Eigenschaften eines natürlichen Monopols näher beschrieben, um im folgenden zu erklären, wann ein natürliches Monopol im Zuge einer dynamischen Weiterentwicklung instabil wird. Schließlich soll das natürliche Monopol im Teilnehmeranschlußbereich unter diesem Gesichtspunkt näher betrachtet werden.

\subsubsection{EIGENSCHAFTEN EINES NATÜRLICHEN MONOPOLS}

Im folgenden wird die Betrachtung eines Ein-Produkt-Monopols vorgenommen. ${ }^{45}$ Die Kennzeichen des natürlichen Monopols sind ein hoher Anteil irreversibler Investitionskosten, also langfristiger Fixkosten, in der Kostenstruktur. Wenn ein Unternehmen die nachgefragte Menge des Produktes billiger herstellen kann als zwei oder mehr Unternehmen, dann handelt es sich um ein natürliches Monopol. Man spricht auch von einer subadditiven Kostenfunktion.

$K(X)$ entspricht den Kosten ${ }^{46}$ eines Unternehmens fur die Produktion von $X$ Outputeinheiten eines Gutes. Werden $X$ Outputeinheiten von $r$ Unternehmen hergestellt, dann produziert jede Firma $h$ einen Output von $x_{h}$. Die Gesamtkosten, die bei der Produktion von $r$ Unternehmen anfallen, lauten

$$
K=\sum_{h=1}^{r} K\left(x_{h}\right) .
$$

Es liegt eine subadditive Kostenfunktion für die Herstellung von $x_{h}$ Einheiten an Output vor, wenn folgende Ungleichung für alle $r \geq 2$ erfullt ist:

$$
K(X)<\sum_{h=1}^{r} K\left(x_{h}\right), \quad \text { wobei } \sum_{h=1}^{r} x_{h}=X,
$$

d.h. wenn der Kostenaufwand für die Herstellung einer bestimmten Outputmenge für ein Unternehmen geringer ist, als wenn $r$ Unternehmen dasselbe Outputni-

Vgl. fur formale Begriffsbestimmungen Baumol et al. (1988), Kap. 7, Sharkey (1982), Kap. 4.

46 Es liegt ein linearer Kostenverlauf zugrunde, $K(X)=k+c X, c>0$. Er kommt der Kostenstruktur im Teilnehmeranschlußbereich näher als ein ertragsgesetzlicher Kostenverlauf. 
veau erwirtschaften. Ungleichung (2.3.1) ist also eine notwendige Bedingung für das Bestehen eines natürlichen Monopols. ${ }^{47}$

Kostensubadditivität steht mit Skalenerträgen und sinkenden Durchschnittskosten im Zusammenhang. ${ }^{48}$ Steigende Skalenerträge beinhalten, daß sich bei einer proportionalen Erhöhung der Inputfaktoren der Output überproportional erhöht. Folglich führen steigende Skalenerträge bei konstanten Inputpreisen zu sinkenden Durchschnittskosten. Dabei beschränken sie sich definitionsgemäß auf den Fall einer Niveauvariation, d.h. einer proportionalen Veränderung aller für den Produktionsprozeß notwendigen Einsatzfaktoren. Durchschnittskosten können dagegen nicht nur aufgrund einer proportionalen Faktorvariation sinken, sondern auch infolge einer nicht-proportionalen Änderung der Einsatzfaktoren wie beispielsweise einer partiellen Veränderung von Inputs oder eines variierenden Faktoreinsatzverhältnisses. ${ }^{49}$

Das Vorhandensein von 'Economies of Scale' bzw. sinkenden Durchschnittskosten impliziert das Bestehen einer subadditiven Kostenfunktion. Allerdings schließt das Vorliegen von Subadditivität nicht die Existenz von 'Economies of Scale' bzw. sinkenden Durchschnittskosten ein. Es kann Subadditivität auch ohne steigende Skalenerträge und nicht sinkenden Durchschnittskosten geben wie an Abbildung 2.3-1 deutlich gemacht werden soll. Dort ist eine fallende langfristige Durchschnittskostenkurve (LDK) dargestellt. Diejenige Kapazität, von der ab die Durschnittskosten nicht mehr sinken, wird als mindestoptimale Betriebsgröße (MOS) bezeichnet. ${ }^{50}$ Obwohl an diesem Punkt die ,Economies of Scale ${ }^{c}$ bereits ausgeschöpft sind und die Durchschnittskosten nicht weiter fallen, liegt hier noch Kostensubadditivität vor. Man spricht auch von einem ,Schwachen Natürlichen Monopol'. Die Situation, in der die LDK bis zur relevanten Menge durchgängig fällt, wird dementsprechend als ,Starkes Natürliches Monopol' bezeichnet. ${ }^{51}$ Durchgängige Skalenerträge sowie langfristige sinkende Durchschnittskosten bis zum relevanten Marktumfang sind also allenfalls hinreichend für Kostensubadditivität, aber keine notwendige Bedingung. ${ }^{52}$

\footnotetext{
Vgl. Sharkey (1982), S. 58.

Bei der Analyse von Mehrproduktunternehmen spielen ebenso Verbundvorteile eine Rolle.

Vgl. Fritsch u.a. (2001), S. 194 f.

Vgl. Kruse (1985), S. 26.

$\mathrm{Zu}$,Starken' und ,Schwachen' Natürlichen Monopolen vgl. auch Kruse (1985), S. 28 f.

Zur formalen Analyse der notwendigen und hinreichenden Bedingung vgl. Sharkey (1982), S.

59 f. Sharkey untersucht dort zudem die Notwendigkeit der strikten Konkavität der Kostenfunktion bzw. der sinkenden langfristigen Grenzkosten in bezug auf das Bestehen von Kostensubadditivität.
} 


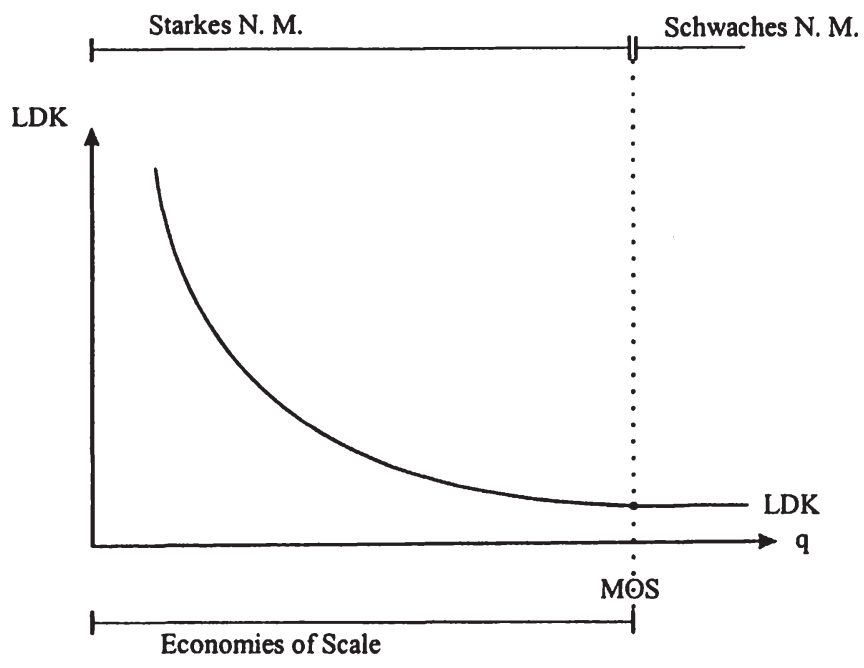

Abbildung 2.3-1: ,Economies of Scale ${ }^{6}$ und Naturliches Monopol ${ }^{53}$

In der Frage, wie stabil ein Natürliches Monopol ist, sind steigende Skalenerträge und sinkende Durchschnittskosten allerdings entscheidend. Liegen sie vor, ist erst die Stabilität eines Natürlichen Monopols gewährleistet. ${ }^{54}$ Liegen sie aber nicht vor, wird dadurch eine Grundlage für potentiellen Wettbewerb gelegt. Das alleinige Vorliegen von Kostensubadditivität impliziert noch keine beständige Marktmacht, weil ein Monopolist durch potentiellen Wettbewerb in bezug auf kosteneffiziente Produktion und Angebot zu kostendeckenden Preisen diszipliniert werden kann. ${ }^{55}$ Nach der Theorie der bestreitbaren Märkte reicht der Wettbewerb von außen in natürlichen Monopolmärkten aus, um zweitbeste Marktergebnisse zu realisieren. ${ }^{56}$ Dabei sind mit zweitbesten Marktergebnissen Preise gemeint, zu denen kein Marktzutritt erfolgt.

In Anlehnung an Kruse (1985), S. 26.

54 Vgl. Braeutigam (1998), S. 1296; Sharkey (1982), S. 87.

5s Zur Theorie der Bestreitbarkeit der Märkte vgl. u.a. Baumol et al. (1988), Fehl (1985), Finsinger (1991), Kruse (1985), Shepherd (1984); Willig (1980).

s6 Vgl. Finsinger (1991), S. 142. Er leitet daraus ab, daß eine staatliche Regulierung eines Natürlichen Monopols unter solchen Gesichtspunkten nicht erforderlich sei. 
Potentieller Wettbewerb ist allerdings nur unter einer Reihe getroffener Annahmen plausibel, nämlich wenn der Marktzutritt ungehindert erfolgen kann $^{57}$ und der Marktaustritt kostenlos ist. Besteht die Notwendigkeit hoher irreversibler Investitionen bei Markteintritt, entstehen bei Marktaustritt Verluste in Höhe der nicht abgeschriebenen Investitionen. Solche versunkenen Kosten stellen eine natürliche Markteintrittsbarriere dar, die potentiellen Wettbewerb behindert. ${ }^{58}$

$\mathrm{Da}$ der Teilnehmeranschlußbereich durch hohe versunkene Kosten gekennzeichnet ist, ist er demnach auf der Infrastrukturebene als Natürliches Monopol nicht angreifbar. Sind die irreversiblen Investitionskosten seitens des Monopolisten bereits getätigt worden und schon abgeschrieben, müssen sie in der Preisgestaltung nicht mehr mit berücksichtigt werden. Die Preisuntergrenze bestimmt sich für den Incumbent nur durch die reversiblen Durchschnittskosten, während ein Newcomer dagegen die irreversiblen Kosten erst aufwenden muß. Dadurch versetzen die versunkenen Kosten den Incumbent in eine strategisch vorteilhafte Position. Nur durch fundamentale Entwicklungen im Hinblick die Nachfrage oder auf den technischen Fortschritt über einen Zeitraum kann ein Natürliches Monopol überwunden werden.

\subsubsection{DYNAMISCHE ASPEKTE NATÜRLICHER MONOPOLE}

Die Stabilität natürlicher Monopole ist dann in Frage gestellt, wenn bei der Analyse Veränderungen im Zeitablauf mit aufgenommen werden. ${ }^{59}$ Dabei ist zu berücksichtigen, daß die Theorie des natürlichen Monopols ein statisches Konzept ist, d.h. ein zeitabhängiger Wandel, der die Stabilität eines natürlichen Monopols gefährden kann, kann nicht modellendogen erklärt werden. Veränderungen exogener Modellparameter sind

- auf einen Nachfrageanstieg zurückzuführen, der eine Rechtsverschiebung der Nachfragefunktion bewirken kann. Skalenerträge können bei der gestiegenen Nachfrage ausgeschöpft sein. Es kann zu Markteintritt neuer Anbieter kommen.

- aus einer Variation kostenbestimmender Faktoren abgeleitet, die eine Verschiebung der Durchschnittskostenkurve nach unten verursachen können. Die-

57 Ungehinderter Marktzutritt erfordert eine Reihe restriktiver Annahmen: Es dürfen keine natürlichen, strategischen oder institutionellen Markteintrittsbarrieren vorliegen, so daß für den Newcomer die Möglichkeit eines hit-and-run-entrys besteht. Zu potentiellen Wettbewerbsmöglichkeiten vgl. außerdem Kap. 3.2.3.3.

Vgl. Burr (1995), S. 67 ff.; Kruse (1985), S. 31 f. 
se ist in der Regel auf technischen Fortschritt zurückzuführen, der vereinfachte Produktionstechnologien ermöglicht oder Faktorpreise senkt.

Zunächst soll auf eine Situation in dem Markt für Telekommunikationsdienste eingegangen werden, der bei steigender Nachfrage im Zeitablauf seine Subadditivität verliert.

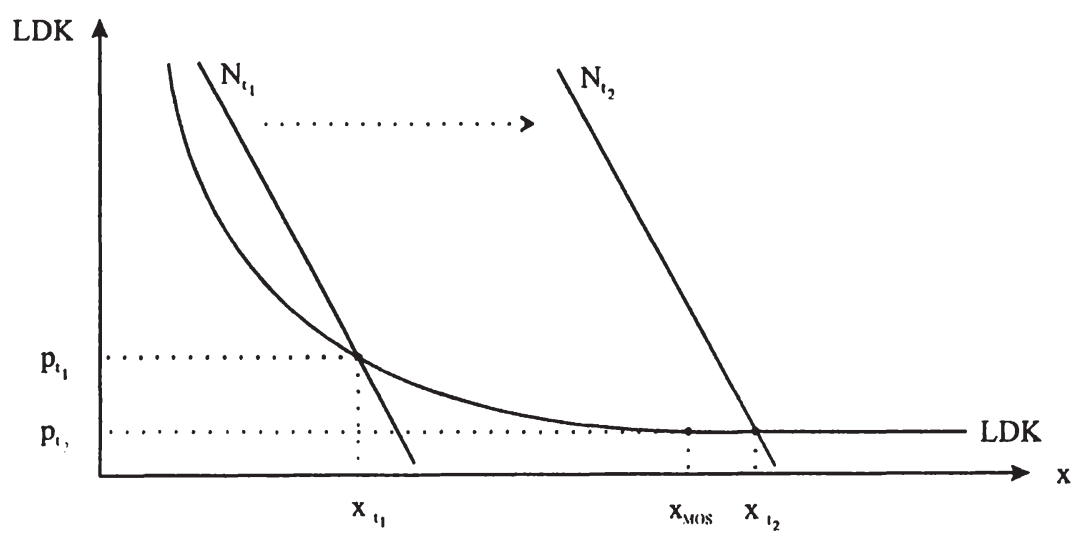

Abbildung 2.3-2: Erosion eines Natürlichen Monopols durch Nachfrageausweitung $^{60}$

In der Abbildung 2.3-2 ist eine sinkende langfristige Durchschnittskostenkurve für eine zunehmende Menge an Outputeinheiten $X$ abgebildet in dem Bereich $0<X<X_{\text {MOS }}$. Dagegen besteht eine Kostensubadditivität für den Bereich $0<X<X_{t_{2}}$. Weiterhin sind zwei Nachfragekurven $N_{t_{1}}, N_{t_{2}}$ dargestellt, wobei sich der Index auf unterschiedliche Zeitpunkte bezieht. Die Verschiebung der Nachfrage von $N_{t_{1}}$ auf $N_{t_{2}}$ ist auf eine exogene Veränderung zurückzufuhren.

Bei $N(p)=N_{t_{1}}$ liegen bei $p_{t_{1}}, X_{t_{1}}$ sinkende Durchschnittskosten und steigende Skalenerträge vor. Kostensubadditivität ist an diesem Punkt noch gegeben. Daraus folgt, daß das Natürliche Monopol stabil ist. Die Nachfrage ist zu gering, als daß sich für einen Newcomer ein Markteintritt lohnen würde, der hohe irreversible Investitionskosten mit sich bringen würde.

Bei $N(p)=N_{t_{2}}$ verläuft die LDK bereits waagerecht. An diesem Punkt sind die steigenden Skalenerträge vollständig abgeschöpft. Führt der Nachfrageschub dazu, daß ein Gleichgewicht bei $X_{t_{2}}$ liegt, dann sind Markteintritte zusätzlicher An-

60 In Anlehnung an Kruse (1985), S. 32 
bieter in diesem Markt denkbar. ${ }^{61}$ Es liegt folglich ein ,Schwaches Natürliches Monopol' vor, das nicht mehr stabil ist, da potentieller Wettbewerb einen Preisdruck ausüben kann.

Desweiteren soll auf eine Situation eingegangen werden, in der die Stabilität eines Natürlichen Monopols durch technischen Fortschritt gefährdet werden kann. Diese ist in Abbildung 2.3-3 dargestellt.

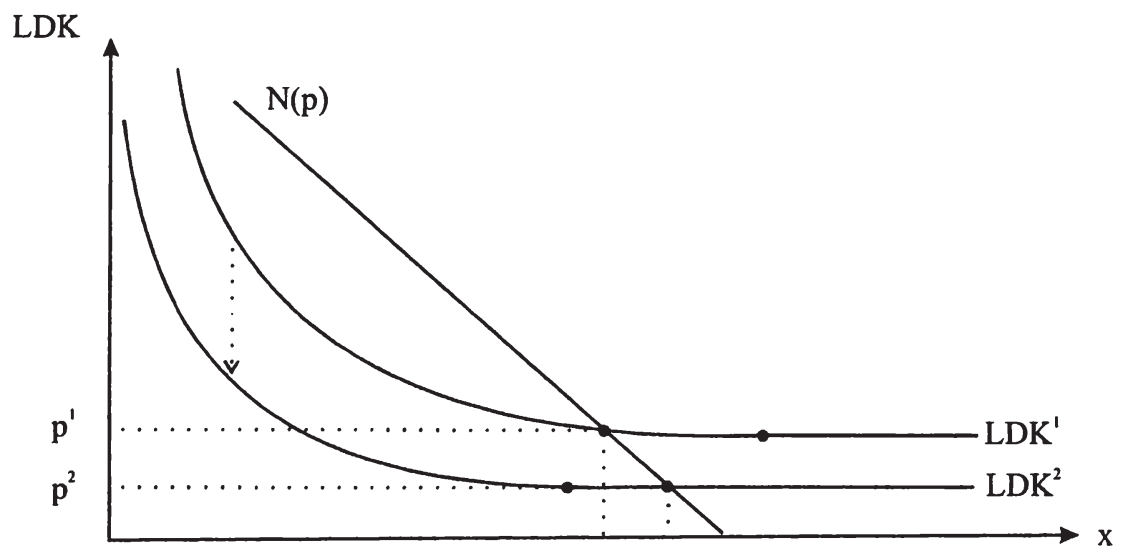

\section{Abbildung 2.3-3: Erosion eines Natürlichen Monopols durch technischen Fortschritt $^{62}$}

Wirkt sich der technische Fortschritt in Form einer Verschiebung der langfristigen Durchschnittskostenkurve von $L D K^{\prime}$ nach $L D K^{2}$ aus, wie in der Abbildung 2.3-3 deutlich wird, wird das Ausschöpfen von Größenvorteilen bei geringeren Outputmengen ermöglicht. Wie schon erwähnt, kann dadurch ein Anreiz für potentielle Anbieter entstehen, in den Markt einzutreten, sofern die Marktaustrittskosten möglichst gering sind. Allerdings entsteht ein Preisdruck aufgrund potentiellen Wettbewerbs nur dann, wenn die $L D K^{2}$ im Schnittpunkt mit der Nachfragefunktion bereits waagerecht verläuft. Ansonsten würde eine Kostensenkung aufgrund technischen Fortschritts die Monopolmacht eher stärken.

Die zentrale Frage in dem hier gegebenen Zusammenhang bezüglich des Teilnehmeranschlußbereichs ist folglich, ob die Markteintrittsbarriere - die versunke-

\footnotetext{
61 Dieser Zusammenhang wird auf den Markt für Telekommunikationsdienste in Kap. 2.3.3 angewendet. 
nen Kosten - mit Hilfe von technischem Fortschritt überwunden werden kann. Diese Frage soll nach einer näheren Betrachtung der technischen Weiterentwicklung im Telekommunikationsbereich im folgenden beantwortet werden.

\subsection{3 ÖKONOMISCHE KONSEQUENZEN DER DIGITALISIERUNG}

Die in Kapitel 2.1.1.3 beschriebenen technischen Weiterentwicklungen auf der Infrastrukturebene haben Auswirkungen sowohl auf die Kostenstruktur des örtlichen Netzbetreibers als auch auf die Nachfrage. Die Konsequenzen, die daraus folgen, sollen anhand der folgenden Abbildung 2.3.-4 erläutert werden.

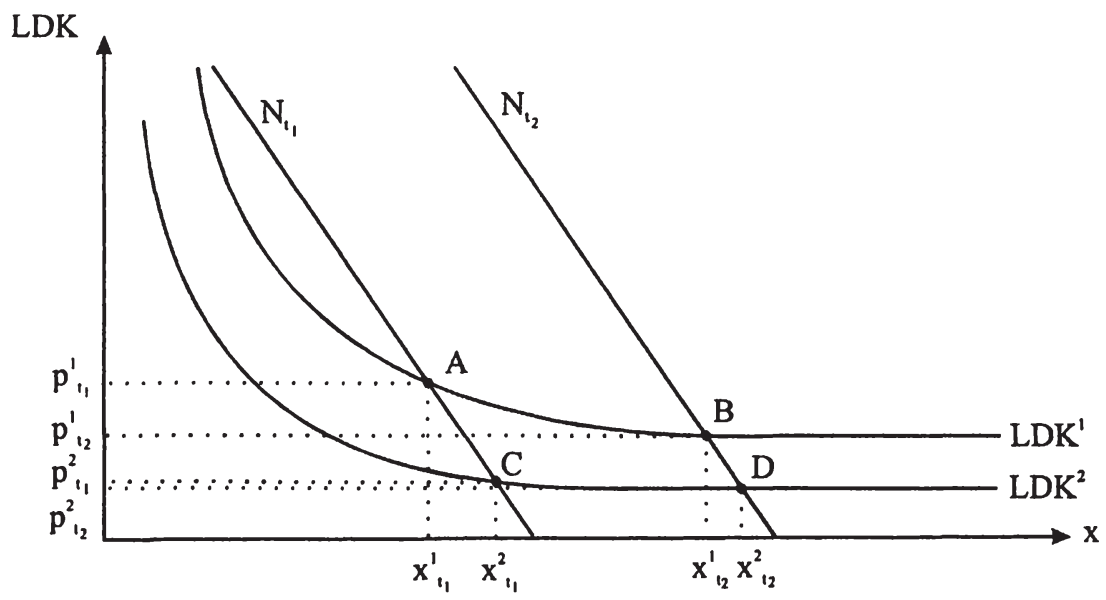

Abbildung 2.3-4: Folgen der Digitalisierung im Festnetzanschlußbereich

In der Abbildung 2.3-4 sind die langfristigen Durchschnittskosten im Festnetzanschlußbereich abgebildet. Die Outputeinheiten $X$ stellen hier ganz allgemein übermittelte Informationseinheiten dar. Hierbei kann es sich um Sprachtelefonie ebenso wie um die Übermittlung von Breitbanddiensten handeln. Dabei sind die $L D K^{1}$ Kosten, die sich auf die digitale Übertragungstechnik beziehen. Die $L D K^{2}$ ergeben sich im Zuge der vollständigen Digitalisierung in bezug auf die Vermittlungstechnik und veranschaulichen die Kostenstruktur für die Übermittlung von 
Informationseinheiten bei Anwendung der neuesten Technologien - also vom heutigen Gesichtspunkt B-ISDN auf der Grundlage der ATM-Technik ${ }^{63}$-.

Die langfristigen Gesamtkosten setzen sich in beiden Szenarien aus einem variablen Kostenblock - den Vermittlungskosten als nutzungsabhängige Kosten - und einem Fixkostenblock zusammen. Die Fixkosten setzen sich wiederum aus einem reversiblen ${ }^{64}$ - Netzwartung, Netzmanagement - und einem irreversiblen Teil Kosten für das Verlegen von Leitungen - zusammen. Außerdem ist ein Nachfragesprung von $N_{t_{1}}$ nach $N_{t_{2}}$ bedingt durch ein erhöhtes Telefonaufkommen abgebildet.

Der Punkt A in Abbildung 2.3-4 stellt den Ausgangspunkt unserer Betrachtung dar und ist vergleichbar mit der Situation des digitalisierten Sprachverkehrs. Es handelt sich dabei entsprechend dem $X_{t_{1}}, p_{t_{1}}$ Schnittpunkt in Abbildung 2.3-2 um ein stabiles Natürliches Monopol.

Bei $\mathbf{B}$ handelt es sich um eine drastische Zunahme der Nachfrage nach Telekommunikationsdienstleistungen, die zunächst nur auf die Erhöhung der Anschlußdichte zurückzuführen ist, beispielsweise durch eine räumliche Markterweiterung, wie sie sich im Zuge der deutsch-deutschen Wiedervereinigung ergeben hat ${ }^{65}$, oder durch das Ansiedeln eines Großkunden oder eines Call-Centers in einer Region. Entsprechend kann das Nachfragewachstum so stark ansteigen, daß die GröBenvorteile im Ortsnetz komplett abgeschöpft werden ${ }^{66}$, so daß die bestehenden Netzkapazitäten vollständig ausgenutzt werden. Dann wäre die Errichtung zusätzlicher Netzkapazitäten in Form von parallel verlegter Infrastruktur notwendig. Dies kann sowohl durch den etablierten Netzbetreiber als auch durch einen alternativen Marktneuling erfolgen. Die Implementierung zusätzlicher Infrastrukturen ist in Teilbereichen von Ortsnetzen, die durch einen hohen Verkehrsbedarf geprägt sind, zu beobachten. Beispielsweise wird das sogenannte Bypassing ${ }^{67}$ praktiziert. In der Regel handelt es sich hierbei um Großkunden, denen ein direkter Anschluß zum Fernnetz ermöglicht wird und damit das Ortsnetz vollständig umgangen wird. Die dafür notwendige Verbindungsstrecke errichtet entweder der Großkunde selbst, Fernnetzbetreiber oder Spezialunternehmen wie Competitive Access Provider.

Der Punkt $\mathbf{C}$ verdeutlicht eine fortschrittsbedingte Reduktion der Kosten infolge einer Digitalisierung der Vermittlungstechnik. Die Digitalisierung des Ortsnetzes

\footnotetext{
63 Vgl. dazu im Anhang A.1.

64 Reversible Fixkosten fallen nicht an, wenn die Produktion eingestellt wird. Irreversible Fixkosten fallen vollkommen unabhängig von der Produktionshöhe an.

65 Die Umsatzrate ist von $1985-90$ von $2,4 \%$ auf $7,6 \%$ von $1990-95$ angestiegen. Vgl. Gerpott (1999), S. 59.

66 Vgl. Kap. 2.3.2.

67 Bypass sind Leitungen privater Netzbetreiber, die das Ortsnetz umgehen und ihre Kunden direkt in den Weitverkehrsbereich weiterleiten. Vgl. hierzu Kap. 3.1.3.
} 
hat bewirkt, daß die Zahl an örtlichen Vermittlungsstellen reduziert wurde, weil in digitalen Ortsnetzen eine OVSt mehrere Anschlußbereiche verwalten kann. Mehrere Ortsnetze wurden deswegen zu einem Regionalnetz zusammengefaßt. Mit der Digitalisierung nehmen neben der Zahl an VSts auch die Zahl der Vermittlungshierarchien ab. Daraus folgt, daß der Bereich der Anschlußleitungen bis zur ersten Vermittlungseinrichtung größer wird. ${ }^{68}$ In der Kostenstruktur wirkt sich diese Entwicklung sowohl auf die variablen Kosten aus, weil sich durch die Einsparung an VSts die Vermittlungskosten pro Anruf - also die nutzungsabhängigen Kostenreduzieren, als auch auf die reversiblen Fixkosten, weil sich der Aufwand in bezug auf Netzwartung und -verwaltung verringert. Mit der Einführung von ISDN wird der Aufbau einer universellen Netzinfrastruktur ermöglicht, die Spezialnetze in ein universelles Netz integriert. Dies wirkt sich in bezug auf Netzverwaltung und Netzmanagement (Betriebs- und Wartungskosten) fixkostenreduzierend aus. Durch eine flexible Integration möglichst vieler Dienste und Verkehrsarten werden die Netzressourcen effektiv genutzt. Aufgrund der variablen Bitratenübertragung beim B-ISDN werden die Kapazitäten effizient ausgelastet. Die paketorientierte Vermittlung erhöht die Ausnutzung der physischen Netzkapazität, weil der Datendurchsatz sehr hoch ist. Es ermöglicht eine höhere Leistungsfähigkeit und ist folglich effizienzsteigernd, weil größere Daten- und Informationsmengen vermittelt werden können. Diese Effizienzsteigerung wirkt sich wiederum durchschnittskostensenkend aus, weil die anfallenden Fixkosten auf ein erhöhtes Output umgelegt werden können. Die Fixkosten für das Verlegen eines alternativen Anschlußnetzes bleiben hingegen unverändert hoch. Da sich alle Kostenkomponenten (anfallende Kosten für Netzverwaltung, und -management, Vermittlungskosten) bis auf den irreversiblen Fixkostenanteil (Verlegen der Kabel) durch die Digitalisierung bei gleichzeitiger Erhöhung des Outputs reduzieren, stellt sich die Frage, ob sich die Durchschnittskosten den Grenzkosten annähern. Da der irreversible Fixkostenanteil für das Verlegen eines Anschlußnetzes ca. $70 \%$ der Gesamtkosten ausmacht ${ }^{69}$, ist es folglich unwahrscheinlich, daß es sich ausschließlich aufgrund der Reduktion der reversiblen und variablen Kosten fur einen Newcomer lohnt, ein zweites Anschlußnetz zu verlegen. Stattdessen wird die Monopolmacht durch den kostenreduzierenden technischen Fortschritt eher gestärkt.

68 Mit der Erweiterung der Digitalisierung auf den Teilnehmeranschluß werden, wie in Kap. 2.2 schon erwähnt, Routing-Funktionen in Richtung des Anschlusses verlagert. Die Folge wird sein, daß sich Ortsnetz und Fernnetz zunehmend weniger voneinander abgrenzen lassen. Aufgrund der Veränderung der Relation zwischen teilnehmerspezifischen und nutzungsabhängigen Kosten kann sich der ökonomische Engpaßbereich des Ortsnetzes verkleinern. Vgl. Albach/Knieps (1997), S. 7.

69 Vgl. Siegmund (1999), S. 214. 
Wie in Abbildung 2.3-4 zu sehen ist, befindet sich $\mathbf{C}$ noch im Bereich sinkender Durchschnittskosten und steigender Skalenerträge.

Die Höhe des Nachfragewachstums ist folglich neben dem Kostensprung für einen Markteintritt entscheidend. Punkt D reflektiert einen solchen Nachfrageschub bei technischem Fortschritt. Durch die Digitalisierung der Übertragungs- und Vermittlungstechnik ist ein deutlicher Anstieg der Nachfrage nach Kommunikationsdiensten zu spüren, weil sich das Spektrum an Diensten vergrößert hat. Man versteht unter Kommunikation nicht mehr nur die herkömmliche Sprachtelefonie, sondern auch Breitbanddienste ${ }^{70}$ sowie Fax und das Internet. Die drastische $\mathrm{Zu}$ nahme des Kommunikationsaufkommens ist vor allem auf den Internet- und Datenverkehr zurückzufuhren. Außerdem ist anzunehmen, daß durch die zunehmende Rechnerunterstützung der Bedarf an Datenkommunikation weiter steigen wird. ${ }^{71}$ Gestiegene Anforderungen seitens der Nutzer an die Kommunikationsnetze und -dienste können den Aufbau einer Infrastruktur parallel zu schon einem bestehenden Netz erfordern: z.B. betreibt COLT Telecom in Frankfurt ein Hochgeschwindigkeits-Ringnetz, das die Standorte der Geschäftsbanken untereinander verbindet. Es zeichnet sich dadurch aus, daß es ein sehr hohes Datenvolumen transportieren kann. ${ }^{72}$ Die Digitalisierung der Vermittlungstechnik auf Grundlage von ATM erlaubt es, alle Telekommunikations- und Mediendienste prinzipiell über Netze wie die terrestrischen, Breitband-, Satelliten-, Mobilfunk- und Telefonnetze anzubieten. Die Netze sind indifferent gegenüber digital aufbereiteten Inhalten und werden auf diesem Weg multifunktional. Die Grenzen zwischen den TIME- Märkte (Telecommunications, Information, Media, Entertainment, Electronics) verschwimmen.

In Zukunft werden im Telekommunikationsmarkt durch das Zusammenwachsen der TIME-Märkte auf der Diensteebene zusätzlich Anbieter auf den Markt treten. Neben Anbietern etablierter Telekommunikationsdienste werden zusätzlich Software-, Medienunternehmen und Internet Service Provider als Anbieter von Basis-

70 Z.B. Pay-TV, Near Video on Demand, Video on Demand, Fernsehtext, Data Broadcasting, Datex-J, Electronic Publishing, Teleshopping, Home-Shopping, Digitales Hörfunksystem, Verkehrsleitsysteme, Fahrgastinformationen in öffentlichen Verkehrsmitteln, Business-TV, Videokonferenzen u.ä. Vgl. Hoffmann-Riem/Schulz/Held (2000), S. 20.

7 Die Zahl an Internet-Nutzer ist von 0,1 Millionen 1991 auf 5-6 Millionen 1998 in Deutschland angestiegen. Vgl. Gerpott (1999), S. 61. Gerpott (1999), S. 64 f. vermutet eine wertmäBige Zunahme um 3,5\% jährlich für Telefondienste und um 9,6\% für Daten- und Mehrwertdienste. Die Regulierungsbehörde hat von 1999-2001 einen Anstieg der Verbindungen ins Internet von 25,167 auf 109, 039 Mrd. Minuten verzeichnet. Vgl. RegTP (2001b), S. 176.

Vgl. dazu auch Kap. 3.3.1. Auf der anderen Seite steht bei der Übertragung kleinerer Datenmengen als bei COLT das technische Aufrüsten bestehender Netze, wie die x-DSL Technologien auf Kupferkabel, gegenüber, das wiederum dem zusätzlichen Verlegen von Infrastruktur ökonomisch widersprechen dürfte. Damit sich der Aufbau eines alternativen Anschlußbereiches lohnt, muß folglich die Durchleitung von sehr hohen Datenmengen nachgefragt werden. 
wie Breitbanddiensten in Frage kommen können. Ebenso treten herkömmliche Telefonbetreiber in andere Märkte ein und bieten Internet-Backbone-Dienste oder Videodienste an. ${ }^{73}$

\subsubsection{FAZIT FÜR DIE ENTWICKLUNG VON WETTBEWERB IM TEILNEHMERANSCHLUBBEREICH}

Es stand die These im Vordergrund, inwieweit das naturliche Monopol im Teilnehmeranschlußbereich im Rahmen einer Neuverkabelung von Anschlüssen mit herkömmlichen Festnetzmedien uberwunden werden kann. Es ist unwahrscheinlich, daß ein exogen bedingter Nachfrageanstieg für eine Überwindung allein ausreichend ist. Ebenso wird das natürliche Monopol auf der Letzten Meile durch den technischen Fortschritt allein nicht instabil. Die bei der Neuverkabelung anfallenden versunkenen Kosten sind so hoch, daß sie eine Markteintrittsbarriere darstellen. Es ist allerdings denkbar, daß technischer Fortschritt im Zusammenhang mit einem starken Nachfrageanstieg, der durch den technischen Fortschritt bedingt sein kann, aber ebenso durch andere Komponenten, einen Markteintritt eines zweiten Anbieters auf der Grundlage einer eigenen Netzinfrastruktur erlaubt. Gerade der Markt fur Telekommunikationsdienste ist zum einen durch rasante technologische Entwicklungen, zum anderen von steigender Nachfrage, die durch technische Veränderungen bedingt ist, geprăgt. D.h. neben dem substitutiven Wettbewerbspotential, wie in Kapitel 3.4 beschrieben, kann sich zusätzlich Wettbewerb durch die technologischen Errungenschaften und die damit einhergehende steigende Nachfrage in der Telekommunikationsbranche entwickeln, bzw. die Entstehung substitutiven Wettbewerbs geht Hand in Hand mit den technischen Entwicklungen in der Telekommunikation durch das Zusammenwachsen der TIME-Märkte.

\subsection{BEWERTUNG INSTITUTIONELLER RAHMENBEDINGUNGEN FÜR DIE EINFÜHRUNG VON WETTBEWERB}

Nachdem die telekommunikationsspezifisch technischen Grundlagen erläutert worden sind und eine ökonomische Interpretation dieser für den Teilnehmeranschlußbereich erfolgt ist, sollen in diesem Kapitel die institutionellen Rahmenbe-

73 Vgl. hierzu die Analyse des Wettbewerbspotentials substitutiver Anschlußtechnologien in Kap. 3.2, 3.3 und 3.4. 
bedingungen, die für Wettbewerb in der Telekommunikation sorgen sollen, vorgestellt und beurteilt werden.

Aufgrund der Situation, daß der deutsche Telekommunikationsmarkt bei der Liberalisierung von der absoluten Marktmacht eines ehemaligen öffentlich geschützten Monopolbetreibers geprägt war, ist abgeleitet worden, daß die allgemeinen Wettbewerbsregeln als Rahmenbedingung für diesen Markt nicht ausreichen. Mit dem Ziel „durch Regulierung im Bereich der Telekommunikation den Wettbewerb zu fördern und flächendeckend angemessene und ausreichende Dienstleistungen zu gewährleisten..." ( $\$ 1 \mathrm{TKG})^{74}$ ist das allgemeine Wettbewerbsrecht um sektorspezifische Regelungen ergänzt worden. Diese Regelungen beinhalten, daß eine Regulierungsbehörde das marktbeherrschende Unternehmen in besonderer Weise regulieren kann. ${ }^{75}$ Das Telekommunikationsgesetz (TKG) liefert die gesetzliche Grundlage für sektorspezifische Regulierungen. Außerdem verabschiedet auf europäischer Ebene das Europäische Parlament sowie der Europäische Rat diverse Richtlinien in bezug auf den Telekommunikationsbereich. Sie sind noch kein unmittelbar geltendes Recht in den Mitgliedsstaaten, sondern müssen erst in nationales Recht umgewandelt werden. So werden sie beispielsweise in Deutschland im TKG umgesetzt.

Die Vorschriften des Gesetzes gegen Wettbewerbsbeschränkungen (GWB) bleiben von der sektorspezifischen Regulierung unberührt, d.h. das allgemeine Wettbewerbsrecht wird vom TKG nicht eingeschränkt. Dagegen knüpfen die wesentlichen Regulierungstatbestände beim TKG bei der Feststellung von Marktbeherrschung an das GWB im Sinne des allgemeinen Wettbewerbsrechts an. Eine Beurteilung dessen erfordert eine adäquate Abgrenzung relevanter Märkte. ${ }^{76}$

Die entscheidenden Regulierungstatbestände sind die Entgeltregulierung, die Regelungen zum Netzzugang (Kap. 2.4.2), die Zusammenschaltung (Kap. 2.4.3) und die Bereitstellung von Universaldiensten (Kap. 2.4.5), ein für das GWB wesensfremder Tatbestand. Auf diese Regulierungstatbestände sowie auf andere institutionelle Rahmenbedingungen, nämlich die institutionellen Markteintrittsbarrieren (Kap.2.4.1) und die Numerierung (Kap. 2.4.4) soll im folgenden näher eingegangen werden. Dabei soll abgewogen werden, ob es tatsächlich um die Formulierung von Rahmenbedingungen geht oder ob es sich um die Verankerung regulierender Eingriffe handelt.

Das Telekommunikationsgesetz (TKG) ist am 01.08.1996 in Kraft getreten.

Vgl. TKG-Entwurf, BT-Drs. 13/3609, S. 33.

Eine ausfuhrliche Marktabgrenzung erfolgt in Kap. 3.2, 3.3. 


\subsubsection{BESEITIGUNG INSTITUTIONELLER MARKTEINTRITTS-}

\section{BARRIEREN}

Unter der Beseitigung institutioneller Markteintrittsbarrieren ist das Gewährleisten einer diskriminierungsfreien Zuweisung von Wegerechten und von knappen Inputfaktoren zu verstehen. Nicht nur die Deutsche Telekom AG (DTAG) verfügt als ehemaliger Monopolbetreiber uber die Wegerechte, sondern auch der Bund ist befugt, öffentliche Wege, Plätze, Brücken, Gewässer für die Errichtung von Festnetzen bzw. Installation von Antennen zu nutzen. Der Bund überträgt dieses Recht unentgeltlich an die Lizenzteilnehmer ( $50 \mathrm{TKG}$ ). Diese Regelung verfolgt das Ziel, jedem Netzbetreiber gleichermaßen das Recht und die Möglichkeit einzuräumen, seine Netzinfrastruktur zu installieren. Es soll vermieden werden, daß der marktbeherrschende Betreiber einen Wettbewerbsvorteil aus der Verfugungsgewalt uber die Wegerechte hat.

Bei dieser Regelung handelt es sich um die Formulierung einer zentralen Rahmenbedingung, die für den Aufbau alternativer Netzinfrastruktur notwendig ist.

Weiterhin sollte für das Ziel der Sicherstellung eines funktionsfähigen Wettbewerbs ein diskriminierungsfreier Zugang zu knappen Inputfaktoren wie z.B. dem Frequenzspektrum gewährleistet werden, die insbesondere für die funkgestützten Anschlußtechnologien wie Wireless Local Loop (WLL) von Bedeutung sind, als auch für den Mobilfunk oder für die Powerline Communication - Technologie (PLC). ${ }^{77}$

Frequenzen haben den Charakter von Allmendegütern. Es handelt sich hierbei um Güter, von denen in der Nutzung niemand ausgeschlossen werden kann, die aber ab einer Kapazitätsgrenze Rivalität im Konsum aufweisen. Sind die Eigentumsrechte an den Frequenzen nicht eindeutig definiert, kann dieser Faktor übernutzt werden, so daß es zu Ineffizienzen kommt. Eine eindeutige Formulierung der Eigentumsrechte bei der Frequenzvergabe kann zu einer allokativ effizienten Nutzung fuhren. ${ }^{78}$ Das TKG sieht in $\$ 11$ hierfür ein Versteigerungsverfahren oder ein Ausschreibungsverfahren vor, das von der Regulierungsbehörde durchgeführt werden soll. Das Ausschreibungsverfahren wurde bei der Frequenzzuteilung zur Nutzung der WLL-Technik im August 1999 versorgungsbereichsbezogen angewendet. ${ }^{79}$ Das Versteigerungsverfahren wurde ebenso bei der Vergabe der UMTSMobilfunklizenzen im Sommer 2000 eingesetzt. In Zukunft werden für eine adäquate Verwendung der PLC-Technologie weitere Frequenzen vergeben werden müssen.

Eine ausführlichere Betrachtung dieser Technologien erfolgt in Kap. 3.4.

Vgl. Distelkamp (1999), S. 93; Merkt (1998), S. $193 \mathrm{ff}$.

Vgl. dazu Kap. 3.4.2.1. 
Die Formulierung eines diskriminierungsfreien Zugangs zu solchen knappen Inputfaktoren wie dem Frequenzspektrum ist als Gewährleistung eines Ordnungsrahmens zu verstehen, das funktionsfähigen Wettbewerb ermöglicht und stabilisiert. Es stellt sich allerdings die Frage, ob der Abbau institutioneller Markteintrittsbedingungen allein ausreicht, um Wettbewerb in einem bisher langjährig geprägten monopolisierten Markt zu ermöglichen. Für Newcomer liegen ungleiche Voraussetzungen vor. Der Incumbent hat einen bedeutenden Wettbewerbsvorsprung aufgrund seiner schon ausgebauten Infrastruktur, hier insbesondere des unmittelbaren Zugangs zum Kunden im Teilnehmeranschlußberich, der als ,Essential Facility' für den Netz- wie für den Dienstebereich bezeichnet werden kann. Daraus folgt, daß der Incumbent aufgrund seiner marktbeherrschenden Stellung Möglichkeiten hat, Newcomern den Markteintritt zu erschweren. Mit der Gewährung von Netzzugang soll den Wettbewerbern der Markteintritt erleichtert werden.

\subsubsection{GEWÄHRUNG VON NETZZUGANG}

\subsubsection{REgelung DES NETZZUGANGS}

Der Begriff Netzzugang beschreibt die Möglichkeit eines Netzbetreibers, auf Teile eines fremden Netzes zuzugreifen, ohne zwingend zugleich Zugriff auf sein eigenes Netz oder Teile desselben zu ermöglichen. Der Netzzugang ist im TKG definiert als ,, die physische und logische Verbindung von Endeinrichtungen oder sonstigen Einrichtungen mit einem Telekommunikationsnetz oder Teilen desselben sowie die physische und logische Verbindung eines Telekommunikationsnetzes mit einem anderen Telekommunikationsnetz oder Teilen desselben zum Zwecke des Zugriffs auf Funktionen dieses Telekommunikationsnetzes oder auf die darüber erbrachten Telekommunikationsleistungen." (\$3 TKG). Dieses Gesetz regelt sowohl den Zugang zwischen Teilnehmeranschluß und dem Netz als auch die Zusammenschaltung von Netzen. Zusammenschaltung ist ein Sonderfall des $\mathrm{Zu}$ gangs und wird im folgenden Abschnitt näher besprochen.

Nach einer Richtlinie der Europäischen Kommission ${ }^{80}$ umfaßt der Ausdruck Netzzugang: ,,Zugang zu Netzkomponenten und zugehörigen Einrichtungen und Diensten, wozu auch der drahtgebundene oder drahtlose Anschluß von Einrichtungen gehören kann; Zugang zu physischen Infrastrukturen wie Gebäuden, Leitungen und Masten; Zugang zu Softwaresystemen [...]; Zugang zur Nummernumsetzung [...]; Zugang zu Mobilfunknetzen [...]; Zugang zu Zugangsberechtigungssystemen für Digitalfernsehdienste."

80 Vgl. Europäische Kommission (2000b), S. 14. 
§33 TKG regelt die Gewährleistung eines diskriminierungsfreien Zugangs „zu seinen intern genutzten und zu seinen am Markt angebotenen Leistungen, soweit sie wesentlich sind, zu den Bedingungen zu ermöglichen, die er sich selbst bei der Nutzung dieser Leistungen ... einräumt, ... “. D.h. die Gewährleistung des Zugangs ist an die ,Essential Facility Doctrine' angelehnt. ${ }^{81}$ Die Regelung des Netzzugangs gewährleistet dem Newcomer auf dem nachgelagerten Markt, der Telefondienste bereitstellen will, aber über keine oder nur geringfügige Netzinfrastruktur verfügt, den Zugang zum Netz des Incumbents auf dem vorgelagerten Markt im Weit- wie im Nahverkehrsbereich.

Bei der Formulierung und Interpretation der Gewährung von Netzzugang werden auf der Grundlage des TKGs regulierende Eingriffe in das Marktgeschehen verankert. Die Regulierungsbehörde sieht es als ihre zentrale Aufgabe an, preisliche Bedingungen mit dem Ziel vorzugeben, Wettbewerb zu fördern und zu stabilisieren. Es finden regelmäßig Entgeltregulierungsverfahren statt. §39 TKG liefert die rechtliche Grundlage für eine Entgeltregulierung für besondere Netzzugänge sowie für Zusammenschaltung. ${ }^{82}$ Bei der Genehmigung von Entgelten ist daruber hinaus die Telekommunikations-Entgeltregulierungsverordnung (TentgV) zu beachten, die auf der Grundlage von $\$ 27$ Abs. 4 TKG erlassen worden ist. Zudem sind materielle Maßstäbe fulr die Entgeltregulierung in \$24 TKG vorgegeben. So müssen sich die Entgelte für das Angebot von Übertragungswegen und der Durchleitung von Telekommunikationsdiensten an den Kosten der effizienten Leistungsbereitstellung orientieren ( $\S 3 \mathrm{Abs} .2 \mathrm{TentgV}) .{ }^{83}$ Voraussetzung furr eine Entgeltregulierung ist das Vorliegen einer marktbeherrschenden Stellung nach §35 TKG und §19 GWB des zu regulierenden Unternehmens. In der Regulierungspraxis betrifft das faktisch nur die DTAG. Bei der Feststellung einer marktbeherrschenden Stellung ist das Einvernehmen des Bundeskartellamtes erforderlich (§82 Abs. 2 TKG).

\subsubsection{ENTBÜNDELUNG VON NETZELEMENTEN INSBESONDERE DER TEILNEHMERANSCHLUBLEITUNG}

Die Möglichkeit der Entbündelung von Netzelementen ist eine entscheidende Bedingung furr die Gewăhrung von Netzzugang und ist in \$2 der Netzzugangsverordnung (NZV) festgehalten: „[Der Betreiber des Telekommunikationsnetzes] hat hierbei entbündelten Zugang zu allen Teilen seines Telekommunikationsnetzes

\footnotetext{
$81 \mathrm{Zu}$ einer ausführlichen ökonomischen Betrachtung von ,Essential Facilities`vgl. Rottenbiller (2002).

82 Danach gelten $\S \S 24,25$ Abs. 1, 3; die $\S \S 27-29,30$ Abs. 1, 3, 6 und $\S 31$ des TKG entsprechend.

83 Vgl. dazu Kap. 5.4 über kostenbasierte Regulierungsansătze.
} 
einschließlich des entbündelten Zugangs zu Teilnehmeranschlußleitungen zu gewähren." Netzbestandteile sollen voneinander entkoppelt werden, so daß ein Wettbewerber nicht ein vorgegebenes Bündel vom etablierten Netzbetreiber mieten oder abnehmen muß, sondern selbst kombinieren kann. Im Prinzip sollen möglichst viele Elemente einzeln anmietbar sein. ${ }^{84}$ Hiermit wird das Ziel verfolgt, dem Newcomer bei der Wahl einer Markteintrittsstrategie möglichst viel Flexibilität einzuräumen.

Die Regelung hinsichtlich des entbündelten Netzzugangs richtet sich wiederum ausschließlich an Anbieter in marktbeherrschender Stellung nach $\$ 19$ GWB. D.h. faktisch unterliegt ausschließlich die DTAG der Entbündelungsregulierung. §35 und $\$ 37$ TKG liefern die Ermächtigungsgrundlage für die Netzzugangsverordnung (NZV). Die NZV regelt die Entbündelung im einzelnen. Von besonderer Bedeutung ist bei der Entbündelung von Netzelementen die entbündelte Teilnehmeranschlußleitung, die es Wettbewerbern ermöglichen kann, Teilnehmeranschlußleitungen anmieten zu können. ${ }^{85}$

Bei Markteintritt in Form des Wiederverkäufer- und Dienstewettbewerbs ${ }^{86}$ sind die neuen Diensteanbieter aufgrund fehlender oder unzureichender eigener Netzinfrastruktur auf das Anmieten von Netzkapazitäten und von Netzanschlüssen angewiesen. Den Dienst Netzanschluß bietet nach Marktöffnung zunächst nur der marktbeherrschende Incumbent an. Ebenso sind alternative Netzbetreiber, die eine eigene Netzinfrastruktur aufbauen, zunächst auf die Anmietung von Anschlüssen angewiesen, da bei Netzaufbau ihre Netzinfrastruktur zunächst sehr klein ist und sie ihre Wettbewerbsposition durch Anmietung von Anschlüssen verstärken können. ${ }^{87}$

84 Denkbare entbündelte Netzbestandteile: Hauptanschluß, Nutzerschnittstellen, Ortsvermittlungen, Tandemvermittlung, Übertragungswege und -leitungen, Signalisierungssysteme, Datenbanken, Betriebsfunktionen, Amtsvermittlung, Rufnummerauskunft. Vgl. Vogelsang (1996c), S. $62 \mathrm{ff}$.

85 Die entbündelte Teilnehmeranschlußleitung impliziert die Bereitstellung einer Übertragungsfunktionalität zwischen Endgerät und dem Abschlußpunkt der Linientechnik (Hauptverteiler) vor dem ersten Konzentrationspunkt, die entweder an einer TVSt oder an einer abgesetzten Konzentratoreinheit liegt.

Der Zugang zur Teilnehmeranschlußleitung stellt einen fortwährenden Konflikt zwischen Incumbent und Regulierungsbehörde dar. Dem Incumbent wird vorgeworfen, Markteintritt durch fehlende Verbindlichkeiten und zu lange Bereitstellungsfristen zu behindern. Vertragsbedingungen zu TAL-Verträgen sind derzeit Gegenstand eines entsprechenden Regulierungsverfahrens. Vgl. RegTP (2001b), S. 106, Pressemitteilung der RegTP vom 12.02.2002. Vgl. dazu Kap. 5.3.1. Vgl. hierzu Kap. 3.1.

87 Der Zugang zur Teilnehmeranschlußleitung stellt einen fortwährenden Konflikt zwischen Incumbent und Regulierungsbehörde dar. Dem Incumbent wird vorgeworfen, Markteintritt durch fehlende Verbindlichkeiten und zu lange Bereitstellungsfristen zu behindern. Vertragsbedingungen zu TAL-Verträgen sind derzeit Gegenstand eines entsprechenden Regulierungs- 
Bisher stehen drei Formen des entbündelten Netzzugangs zur Verfügung. ${ }^{88}$ Diese drei Formen sind seit Dezember 2000 als verbindliche Rechtsgrundlage von dem Europäischen Parlament und dem Europäischen Rat verabschiedet worden.

\section{Full Unbundling}

Ein Newcomer kann ausgewählte Anschlußleitungen komplett zu einem von der Regulierungsbehörde vorgegebenen Mietpreis übernehmen. Die komplette Anmietung von Teilnehmeranschlußleitungen erfolgt vom Hauptverteiler aus. Sie setzt eine gemeinsame Nutzung von technischen Räumen und den Zugang zum Hauptverteiler in der TVSt der DTAG voraus (Kollokation). Das Full Unbundling ist folglich für die Wettbewerber interessant, deren Infrastruktur bis an den Hauptverteiler heranreicht. Der ausgewählte Teilnehmeranschlußbereich wird aus dem Netz des Incumbents ausgegliedert und in das Netz des Wettbewerbers eingebunden. Auf diese Weise verliert der etablierte Anbieter die Möglichkeit, den bisherigen Kunden mit Diensten zu versorgen. Das Full Unbundling wird gegenwärtig in Deutschland für eine begrenzte Anzahl an Teilnehmeranschlüssen praktiziert. ${ }^{89}$ Die Preise der entbündelten Anschlußleitung unterliegen der Einzelpreisregulierung. ${ }^{90}$ Die deutsche Regulierungsbehörde für Telekommunikation und Post (RegTP) hat den monatlichen Mietpreis eines herkömmlichen Kupferdoppelader- Teilnehmeranschlusses sowie einmalige Bereitstellungs- und Kündigungspreise auf der Grundlage der, Forward Looking - Long Run Average Incremental Cost ' Methode (FL LRAIC) festgelegt. ${ }^{91}$

\section{Line-Sharing}

Beim Line-Sharing teilen sich das regulierte Unternehmen und der Wettbewerber dieselbe Anschlußleitung, bieten aber unterschiedliche Produkte an. Der Incumbent erbringt weiter die reine Sprachtelefonie (Grunddienste). Der Newcomer rüstet die Kupferkabel mittels eigener x-DSL-Modems auf und bietet über die Anschlußleitung des Incumbents ausschließlich breitbandige Datendienste an, wie $z$. B. einen Internetzugang. ${ }^{92}$ Das Line-Sharing vollzieht eine Aufteilung des Mark-

verfahrens. Vgl. RegTP (2001b), S. 106, Pressemitteilung der RegTP vom 12.02.2002. Vgl. dazu Kap. 5.3.1. Vgl. Europäische Kommission (2000a). Zu Unbundling vgl. auch Gabelmann (2001), Hökkels (2001a), Merkt (1997). Vgl. Europăische Kommission (2000b), S. 22. Ebenso wird das Full Unbundling in 6 weiteren EU-Ländern angewendet. ve/Prosperetti (2000), S.13. Zur Einzelpreisregulierung vgl. Kap. 5.1 und Kap. 5.2. Vgl. Monopolkommission (2001), S. 73 f. Eine ausführliche Erklärung des FL LRAIC Ansatzes sowie die Festlegung von Miet- und Bereitstellungspreisen erfolgt in Kap. 5.4 sowie Kap. 6.3.3.

$92 \mathrm{Zu}$ technischen Einzelheiten vgl. Kap. 2.1.1.3. 
tes nach Produkten. Der Incumbent soll reine Sprachtelefonie - also Schmalbanddienste - anbieten, der Entrant soll Breitbanddaten vermitteln. Wird aber in $\mathrm{Zu}$ kunft Internet-Telefonie über Datenverkehr qualitativ gleichwertig möglich sein, dann wird technischer Fortschritt dazu führen, daß die Grenze zwischen den Produkten verschwimmt. ${ }^{93}$ Der Newcomer wird ebenso Sprachdienste anbieten können. Auf diese Weise verändert sich die Wettbewerbsposition. Es bleibt zu hinterfragen, ob auf dieser Grundlage das Line-Sharing noch sinnvoll ist.

\section{Bitstream Access}

Beim Bitstream Access rüstet der Incumbent selbst sein Netz mit $x$-DSL oder mit Glasfaser auf. Dem Entrant gewährt er Zugang zu einer bestimmten Übertragungsbandbreite. Die reine Sprachtelefonie wird weiterhin nur vom Incumbent angeboten, Breitbanddienste dagegen sollen sowohl von dem Incumbent als auch von den Wettbewerbern bereitgestellt werden. Die Wettbewerber agieren hier allerdings nur als Wiederverkäufer. Der Bitstream Access wird derzeit in acht EULändern praktiziert, darunter auch in Deutschland.

\subsubsection{BEWERTUNG DER NETZZUGANGSREGELUNG}

Die Bewertung soll zum einen vom Standpunkt einer sehr jungen Wettbewerbsphase, zum andern von einer reifen Marktphase vollzogen werden.

Bei Einführung von Wettbewerb im Teilnehmeranschlußbereich kann die Entbündelungsverpflichtung als eine hilfreiche Maßnahme furr seine Etablierung betrachtet werden. Die konkrete Ausgestaltung dieser Regelung beinhaltet allerdings zum Teil massive regulatorische Eingriffe in den Markt, wie z. B. die konkreten Preisvorgaben von Teilnehmeranschlüssen oder der Entgeltregulierung fur das Angebot von Übertragungswegen und Sprachdiensten. Die EU-Kommission befurwortet in einem Richtlinienvorschlag ${ }^{94}$ nach wie vor bereichsspezifische Regulierung besonders in Branchen, in denen ehemalige Monopolbetreiber nach wie vor eine starke Marktmachtposition bekleiden. Für diese Situation will sie klare Kriterien für rechtliche Eingriffe formulieren. In diesem Fall sollen regulierende Maßnahmen für die Gewährleistung von Zugang und Zusammenschaltung vorgeschrieben werden mit der Begründung, der ehemalige Monopolbetreiber habe eine marktbeherrschende Stellung und eine dominante Verhandlungsposition. Die regulierenden Eingriffe reichen von dem Sicherstellen des Zugangs für den konkur-

\footnotetext{
93 Vgl. dazu Kap. 3.4.1.3.

94 Vgl. Europäische Kommission (2000b).
} 
rierenden Betreiber zu dem Netz des anderen Betreibers ${ }^{95}$ bis hin zu Preiskontrollen und Entgeltvorgaben, nămlich die Verpflichtung, daß die Entgelte für die Gewăhrung des Netzzugangs und der Zusammenschaltung kostenorientiert sind. Dies impliziert eine Einsicht in die Buchhaltung des ehemaligen Monopolbetreibers seitens der Regulierungsbehörde für den Nachweis von Kostenstrukturen. Es stellt sich die Frage, inwieweit solche Eingriffe wie Preisvorgaben und Entgeltregulierung für die Sicherstellung von Wettbewerb gerechtfertigt sind. In Kapitel 5 soll sich intensiv mit dem Problem des Zugangsentgelts befaßt werden.

In einer reifen Marktphase infrastrukturbasierten Wettbewerbs ${ }^{96}$ scheint eine Entbündelungsverpflichtung nicht gerechtfertigt. Der Teilnehmeranschlußbereich verfugt über keine Bottleneck- Eigenschaften mehr. Es liegt keine ,Essential Facility" vor. Eine Entgeltregulierung ist nach dem Argument der Regulierung eines marktbeherrschenden Unternehmens nicht mehr zu verantworten. Ein Richtlinienvorschlag der EU-Kommission ${ }^{97}$ spricht sich bei reifem Wettbewerb ebenso gegen eine Regulierung aus. Stattdessen stützt er sich auf die Prämisse, daß allgemeine Wettbewerbsregeln gelten sollen. Den betroffenen Netzbetreibern wird die Freiheit gelassen, ihre Bedingungen für die Gewährung von Netzzugang und $\mathrm{Zu}$ sammenschaltung auszuhandeln. Allerdings kann es bei einer Verhandlungslösung zu kollusivem Verhalten zwischen den Verhandlungspartnern kommen. Hieraus kann wiederum die Frage abgeleitet werden, ob regulierend eingegriffen werden sollte oder nicht. In Kapitel 5.3 wird sich mit diesem Problem ausfuhrlich beschäftigt.

\subsubsection{ZUSAMMENSCHALTUNG (INTERCONNECTION)}

Bei der Zusammenschaltung handelt es sich um ,die physische und logische Verbindung öffentlicher elektronischer Kommunikationsnetze, die von demselben oder einem anderen Unternehmen genutzt werden, um Nutzern eines Unternehmens die Kommunikation mit Nutzern desselben oder eines anderen Unternehmens oder den Zugang $z u$ den von einem anderen Unternehmen angebotenen Diensten zu ermöglichen. " ${ }^{98}$ Bei der Zusammenschaltung von Netzen geht es um einen Spezialfall des Netzzugangs, nämlich einen reziproken Netzzugang, der in beide Richtungen vollzogen wird. Die Reziprozität bedeutet ökonomisch, daß je-

\footnotetext{
95 Mitgliedstaaten sind aufgefordert worden, eine Regulierung bezüglich des entbündelten $\mathrm{Zu}$ gangs zum Teinehmeranschlußbereich bis Ende 2000 einzufuhren (,enforcement action'). Vgl. Cave/Prosperetti (2000), S. 14.

96 Eine Erläuterung des Begriffs erfolgt in Kap. 3.1.3.

97 Vgl. Europăische Kommission (2000b).

$98 \mathrm{Vgl}$. Europäische Kommission (2000b), S. 15.
} 
der Netzbetreiber zugleich Käufer und Verkäufer von Dienstleistungen ist . ${ }^{99}$ Physikalisch impliziert eine Zusammenschaltung die Etablierung einer HardwareSchnittstelle zwischen Netzen, die die Verbindung zwischen Teilnehmern verschiedener Netze ermöglicht.

Die Zusammenschaltung von Netzen kann ebenso als Lösung zur Internalisierung der positiven Netzexternalitäten bezeichnet werden. ${ }^{100}$ Es gibt wegen der Existenz von Netzexternalitäten ein grundlegendes Interesse der Netzanbieter, Interconnection freiwillig zu vereinbaren. Der Anreiz, sich zusammenzuschalten, wird zunehmend geringer, je größer ein Netzbetreiber ist und je kleiner alle anderen sind. Im TKG ist eine generelle Zusammenschaltungspflicht für Betreiber öffentlicher Kommunikationsnetze ( $§ 36$ TKG) festgeschrieben. Jedes Unternehmen ist verpflichtet, auf entsprechende Nachfrage eines Netzbetreibers ein Angebot über die Zusammenschaltung abzugeben und mit dem Ziel auf eine Einigung zu verhandeln. Scheitern die Verhandlungen, ordnet die Regulierungsbehörde eine Netzzusammenschaltung gemäß $\$ 37$ TKG an.

In der Regulierungspraxis dagegen wird die Regulierungsbehörde fast ausschließlich von den Wettbewerbern auf Anordnung der Netzzusammenschaltung mit der $D T A G$ angerufen, da die DTAG über das umfassenste und flächendeckenste Telekommunikationsnetz verfügt, in dem $98 \%$ aller Festnetzanschlüsse realisiert sind. Als marktbeherrschendes Unternehmen unterliegt die DTAG gemäß $§ 19$ GWB besonderen Verpflichtungen, die in der NZV festgehalten sind.

Die RegTP ist bei der inhaltlichen Ausgestaltung von Zusammenschaltungsanordnungen an das TKG insbesondere an $\S 37$ TKG gebunden. ${ }^{101}$ Die konkrete Ausgestaltung der Zusammenschaltungsbedingungen liegt im Gestaltungsspielraum der Regulierungsbehörde, orientiert sich aber an Verträgen, auf die sich Marktteilnehmer freiwillig geeinigt haben. Die Regulierungsbehörde ist der Rechtsauffassung, daß sie nach $\$ 37$ TKG auch umstritten gebliebene Entgelte anordnen kann, was rechtlich nicht eindeutig ist. ${ }^{102}$ Dennoch kann sie in einem gesonderten Entgeltregulierungsverfahren nach $\$ 39$ TKG Zusammenschaltungsentgelte festlegen. Von 1999 bis zum 31.01.2001 lag in Deutschland eine Einzelpreisregulierung der Netzzusammenschaltungsentgelte für vier Entfernungsstufen vor (City, Regio für

$99 \mathrm{Vgl.} \mathrm{WIK/EAC} \mathrm{(1994),} \mathrm{S.} 55$.

100 Vgl. hierzu Kap. 4.

$101 \mathrm{Zu}$ den Zusammenschaltungsverfahren von den Jahren 1999 bis 2001 vgl. RegTP (2001b), S. $84 \mathrm{ff}$.

102 Die Regulierungsbehörde hat Entgelte bei dem Zusammenschaltungsverfahren vom 8. Sept. 2000 zwischen Mannesmann Arcor und DTAG im Rahmen der Element Based Charging (EBC) - Diskussion angeordnet. Das Verfahren ist allerdings vom Verwaltungsgericht Köln und von dem OVG Münster zurückgewiesen worden mit der Begründung, die Entgelte hätten in einem gesonderten Entgeltgenehmigungsverfahren nach §39 TKG festgelegt werden müssen. 
$<50 \mathrm{~km}$, Regio für $<200 \mathrm{~km}$, Fernzone). Die Festlegung dieser Tarife erfolgte auf Grundlage der ,Long Run Incremental Costs ' (LRIC). ${ }^{103}$ Seit dem 1.1.2002 basiert die Festlegung der Entgelte auf einem Zonenmodell. Die Bestimmung des Entgelts orientiert sich dann an der Zahl der genutzten Netzelemente, dem ,Element Based Charging' (EBC). Die Verbindungen sollen nach ihrem Durchlauf an Vermittlungsstellen bepreist werden und nicht mehr nach ihrer Entfernung. ${ }^{104}$ Die Zusammenschaltungsverpflichtung läßt sich im Hinblick auf die Reduzierung der Netzexternalitäten ökonomisch rechtfertigen und als Rahmenbedingung für die Sicherstellung von Wettbewerb bezeichnen. Die genauen Entgeltvorgaben furr die Zusammenschaltung entsprechen wiederum einem regulatorischen Eingriff in das Marktgeschehen, der sich pauschal nicht rechtfertigen läßt. In Kapitel 5.3 wird die Zusammenschaltung von Netzbetreibern näher analysiert.

\subsubsection{NUMERIERUNG}

Unter dem Begriff Numerierung ist sowohl Nummernverfügbarkeit für Anschlüsse als auch Nummernportabilität gemeint. Für die Strukturierung, Ausgestaltung und Verwaltung des Nummernraumes ist die Regulierungsbehörde zuständig ( $\$ 43 \mathrm{TKG})$ mit dem Ziel ,jederzeit den Anforderungen von Nutzern, Betreibern von Telekommunikationsnetzen und Anbietern von Telekommunikationsdienstleistungen zu genügen" (\$43 (1) TKG).

Unter Nummernportabilität sind drei Formen zu unterscheiden:

1. Netzbetreiberportabilität bezeichnet die Übertragbarkeit der Nummer bei einem Wechsel eines Konsumenten zu einem alternativen Anschlußnetzbetreiber.

2. Diensteportabilität bezieht sich auf die Übertragbarkeit der Nummer bei Wechsel des Dienstes z.B. von einem herkömmlichen Telefonsystem $\left(\right.$ POTS $^{105}$ ) zu einem ISDN - Anschluß.

3. Räumliche Portabilităt beinhaltet die Beibehaltung der alten Rufnummer bei Umzug ${ }^{106}$ eines Telefonteilnehmers in eine andere Stadt oder Region.

Die Netzbetreiberportabilität ist das wichtigste Element bei der Einführung von Wettbewerb im Teilnehmeranschlußbereich. Wird sie von dem marktbeherr-

\footnotetext{
103 Vgl. RegTP (2001b), S. 73 sowie Distelkamp (1999), S. 91. Europäische InterconnectionTarife im Vergleich vgl.: http://www.ispo.cec.be unter Interconnection Tariffs in Member States as of $1^{\text {st }}$ March 2000. Zu LRIC vgl. dazu Kap. 5.4.1.2. Vgl. hierzu Zusammenschaltungsleistungen in Kap. 3.3.3.1. EBC Tarife nachlesbar unter RegTP (2001b), S.77.

105 POTS - Plain Old Telephone System/Service

106 Vgl. Reinke (1998), S. 74 ff.
} 
schenden Netzbetreiber verweigert, stellt sie für alle anderen Netzanbieter eine Markteintrittsbarriere dar. Ein Anbieterwechsel mit Rufnummeränderung bedeutet für die Teilnehmer hohe Transaktionskosten. Nach einer Gallup-Umfrage von 1994 in den USA würden von allen Geschäftskunden $90 \%$ und $80 \%$ aller Privatkunden nicht den Betreiber wechseln, wenn sie ihre Telefonnummer dafür ändern müßten. ${ }^{107}$

Das Numerierungssystem mußte in Deutschland vor der Digitalisierung an die Forderungen des mechanischen EMD-Wahlverfahrens ${ }^{108}$ angepaßt sein und basierte auf einem strikten hierarchischen Netzaufbau und entsprechender Numerierung, d.h. bei Verbindungsaufbau wurden die Wählziffern nacheinander ausgewertet. Die digitale Vermittlungstechnik erlaubt inzwischen eine größere Flexibilität der Nummernverwaltung. Zukünftig wird es ,intelligente Netzarchitekturen geben, die Zuordnungsinformationen in zentralen Datenbanken speichern. Dann wird technisch auch das ,personal numbering' möglich sein, d.h. die Zuweisung einer Rufnummer ohne räumliche oder netzbezogene Portabilitätsbeschränkung. Im TKG ist die Verpflichtung zur Sicherstellung von Netzbetreiberportabilität festgeschrieben. In $\S 20$ Abs. 2 S. 3 der Telekommunikations- Kundenschutzverordnung wird festgehalten, daß der Kunde ein eigenständiges dauerhaftes Nutzungsrecht an seiner Rufnummer hat. Ein Betreiberwechsel kommt einer Kündigung gleich. Ein Betreiber kann für den Wechsel kein Entgelt verlangen. Da der Betreiber aber dazu angehalten ist, technische Vorkehrungen zu schaffen, so daß die Rufnummernmitnahme sichergestellt ist, kann er die dadurch anfallenden Kosten im Rahmen von Entgeltanträgen geltend machen. ${ }^{109}$

Für die Etablierung von Wettbewerb muß sowohl Nummernverfügbarkeit für Anschlüsse als auch Nummernportabilität gewährleistet sein.

\subsubsection{UNIVERSALDIENSTVERPFLICHTUNG}

Die Universaldienstverpflichtung ist in $\S 17$ des TKG geregelt. Es handelt sich hierbei um ein „Mindestangebot an Telekommunikationsdienstleistungen.., [das] für eine bestimmte Qualität festgelegt ist und zu denen alle Nutzer unabhängig von ihrem Wohn- oder Geschäftsort zu einem erschwinglichen Preis Zugang haben müssen ". Aus dieser Formulierung wird deutlich, daß Telekommunikationsdienstleistungen als Grundversorgung für die Öffentlichkeit gesehen werden. Dar-

107 Vgl. http://www.nortelnetworks.com/cool/np/survey.html .

108 EMD - Edelmotordrehwähler . Vgl hierzu Siegmund (1999), S. 95 ff.

109 Vgl. Distelkamp (1999), S. 92. 
aus folgt, daß Anschlußpreise nicht entsprechend ihren tatsächlichen Kosten differenziert werden dürfen. Anschlußanträge können nicht abgelehnt werden.

$\mathrm{Zu}$ dem Universaldienst sind alle Unternehmen verpflichtet, die entweder eine marktbeherrschende Stellung nach § 22 GWB auf dem răumlich relevanten Markt einnehmen oder aber die einen Umsatzanteil von mehr als $4 \%$ des sachlich relevanten Marktes verfulgen.

Die Universaldienstverpflichtung läßt sich nicht als ökonomisch notwendige Rahmenbedingung für die Sicherstellung von Wettbewerb bezeichnen. Es ist viel mehr eine aus politischen Gründen gewollte Bedingung, deren Finanzierung aus diesem Grund sich nicht über Marktmechanismen ergibt, sondern geregelt werden muß. ${ }^{110}$

$110 \mathrm{Zu}$ den Wohlfahrtseffekten einer vollständigen Netzabdeckung im Sinne einer Universaldienstverpflichtung vgl. Kap. 4.2. 
Sarah Eliza Schniewindt - 978-3-631-75130-5 Downloaded from PubFactory at 01/11/2019 07:57:00AM via free access 
Aus der Beschreibung der technischen Grundlagen ging hervor, daß sich aus der Sektorstruktur in der Telekommunikation eine Einteilung in vorgelagerte Märkte die Netzinfrastruktur sowie Vorleistungen- und nachgelagerte Mărkte - die Telekommunikationsdienste - als sinnvoll erweist. ' Die Funktionsfahigkeit des nachgelagerten Marktes ist dabei von dem vorgelagerten Markt abhängig. Bei der Analyse von möglichen Markteintritten in den Anschluß- wie Fernnetzbereichen ist hervorzuheben, daß ein Markteintritt sowohl auf den vorgelagerten Märkten als auch nur auf den nachgelagerten Märkten erfolgen kann. Bei einem Markteintritt ausschließlich auf einem nachgelagerten Markt spricht man vom Wiederverkăuferwettbewerb. Findet ein Markteintritt auch auf dem vorgelagerten Markt statt, spricht man vom Dienste- bzw. infrastrukturbasierten Wettbewerb.

Um eine adäquate Marktabgrenzung vorzunehmen, ist es sinnvoll, zunächst die drei verschiedenen Formen des Markteintritts vorzustellen (Kap. 3.1). Darauf soll eine Bestimmung des relevanten Marktes folgen. Sie ist erforderlich, um die Marktstruktur sowie die Wettbewerbsverhältnisse beurteilen zu können. Das Verfahren einer sinnvollen Marktabgrenzung hängt von ihrer Zielsetzung ab. Aus diesem Grund soll in dem Kapitel 3.2 die Zielsetzung der Marktabgrenzung mittels relevanter Kriterien allgemein diskutiert werden, bevor die eigentliche anhand des Telekommunikationssektors in Kap. 3.3 vorgenommen wird. Sie soll die Verhältnisse infrastrukturbasierten Wettbewerbs beurteilen. In Kapitel 3.4 soll insbesondere auf das Wettbewerbspotential im Anschlußbereich eingegangen werden. Zunächst werden alternative Anschlußtechnologien vorgestellt bevor das in Kap. 3.2 vorgestellte Marktabgrenzungskonzept auf sie angewendet wird.

\subsection{WETTBEWERBSFORMEN DES MARKTEINTRITTS}

Im folgenden werden drei Wettbewerbsformen vorgestellt, die sich hinsichtlich des Aufbaus bzw. Besitzes eigener Netzinfrastruktur auf Seiten des/der Wettbewerber graduell unterscheiden und sowohl im Orts- wie im Weitverkehrsbereich auftreten. Hierbei sei darauf hingewiesen, daß eine Unterscheidung hinsichtlich der Wettbewerbsformen auf funktionaler Ebene sinnvoll ist, obwohl sie auf organisatorischer Ebene nicht möglich ist, weil Unternehmen sowohl auf eigenen Netzen als auch auf der Infrastruktur anderer Unternehmen Dienste betreiben. Im TKG findet sich keine Aussage über den Stellenwert der verschiedenen Wettbewerbsformen.

Vgl. dazu Kap. 2.1 sowie Abbildung 2.1.-3. 


\subsubsection{WIEDERVERKÄUFERWETTBEWERB (,RESALE COMPETITION“)}

Der Wiederverkäuferwettbewerb findet ausschließlich auf dem nachgelagerten Markt statt. Ein Wiederverkäufer mietet Infrastruktur von dem Incumbent an und veräußert vom Incumbent zu Großhandelskonditionen erworbene Dienste an den Endkunden weiter. Dabei wird ein Teil der Großhandelsrabatte an den Kunden weitergegeben. Der Wiederverkäuferwettbewerb zeichnet sich also dadurch aus, daß ein Wettbewerber keine eigene Infrastruktur besitzen muß, um als Wiederverkäufer von Telekommunikationsdiensten aufzutreten. Er agiert als Zwischenhändler auf dem nachgelagerten Markt für Telekommunikationsdienste. Sein Gewinn ergibt sich aus der Differenz von Einkaufspreis und Verkaufspreis und entspricht einem Arbitragegeschäft.

Der entscheidende Vorteil von Wiederverkäuferwettbewerb ist, daß für einen Wettbewerber ein schneller Marktein- und austritt möglich ist, ohne hohe irreversible Investitionskosten tätigen zu müssen. ,Resale Competition' hat dagegen den Nachteil, daß sich ein Wiederverkäufer in Abhängigkeit von dem Incumbent befindet, der nach wie vor den vorgelagerten Markt beherrscht. Dies ist insbesondere bei der Entscheidung der Bestimmung eines Entgelts für die Gewährung des Zugangs zu der notwendigen Infrastruktur ein Problem. ${ }^{2}$ Bei der Liberalisierung wurde davon ausgegangen, daß der Incumbent prinzipiell kein Interesse daran habe, Wettbewerber auf den Markt zu lassen. Aus diesem Grund wird der Incumbent - hier die DTAG -gesetzlich dazu verpflichtet, den Wiederverkauf seiner Leistungen zuzulassen. ${ }^{3}$ Außerdem muß die DTAG ihre festgelegten Entgelte für den Wiederverkauf von Leistungen von der deutschen RegTP genehmigen lassen. ${ }^{4}$

Vom Wiederverkäuferwettbewerb gehen keine wesentlichen wettbewerbsinduzierten Impulse auf die Preisentwicklung von Telekommunikationsdiensten aus. Es wird nur die Kette zwischen Anbieter und Nachfrager verlängert. Wiederverkäuferwettbewerb kann einen Wettbewerb im vorgelagerten Markt zwischen Netzbetreibern nicht ersetzen, kann ihn aber als ergänzende Markteintrittsstrategie verstärken. Auf diese Weise kann ein Newcomer zunächst ein Produkt im Markt plazieren und sich einen Namen machen, bevor er ein erhebliches Investitionsrisiko auf sich nimmt und eine eigene Infrastruktur beginnt aufzubauen.

Im Vergleich zu den anderen Wettbewerbsformen kommt der Wiederverkäuferwettbewerb eher selten vor, weil nur ganz geringe Netzinvestitionen notwendig

2 Vgl. dazu Kap. 5.

3 „Ein marktbeherrschendes Unternehmen kann dazu gezwungen werden, Vertriebsbeziehungen mit Unternehmen aufzunehmen, die zu ihm in Wettbewerb treten wollen." $\$ 33$ Abs. 1 TKG. Vgl. Büchner u.a. (1997), RN 17 f.

$4 \quad$ Vgl. Pressemitteilung der RegTP vom 08.09.1999. 
sind, damit ein Wettbewerber definitionsgemäß als Netzbetreiber gilt und man dann von Dienstewettbewerb sprechen würde.

\subsubsection{DiensteWeTtBeWERB (,SERVICE COMPETITION“)}

Beim Dienstewettbewerb kombinieren Wettbewerber erworbene Leistungen mit neuen Merkmalen auf Basis angemieteter Leitungen und veräußern diese weiter (z.B. Corporate Networks oder Bereitstellen spezieller Dienste wie Anrufbeantworterfunktionen ${ }^{5}$ ). Dabei erfordert das Hinzufügen neuer Leistungsmerkmale den Besitz über eine minimale eigene Infrastruktur. Dies kőnnen Netzbestandteile im engeren Sinn (wie z.B. Übertragungs- und Vermittlungseinrichtungen) oder ebenso Switches, Rechner oder Speichermedien für eine Fernsprechauskunft sein. Die entscheidende Voraussetzung für die Funktionsfähigkeit von Dienstewettbewerb ist, daß einzelne Netzelemente angemietet werden können. Steht beispielsweise im Teilnehmeranschlußbereich nur der Anschluß des etablierten Netzbetreibers zur Verfügung (One-Way-Access), dann ist der Newcomer auf das Anmieten von entbündelten Teilnehmeranschlußleitungen angewiesen. ${ }^{6}$

Der Dienstewettbewerb eignet sich wie der Wiederverkäuferwettbewerb als ergänzende Strategie zu netzinfrastrukturellem Wettbewerb. Dabei sind die Übergänge zwischen Dienste- und infrastrukturbasiertem Wettbewerb fließend. Ein stark ausgebauter Dienstewettbewerb kann schon der Beginn von Wettbewerb auf der Infrastrukturebene bedeuten. Der Newcomer befindet sich dabei in bezug auf die Funktionsfähigkeit seines Netzes noch in einer Abhängigkeit gegenüber dem Incumbent, weil er weitestgehend auf den Zugang zur Infrastruktur des Incumbents angewiesen ist.

\subsubsection{INFRASTRUKTURBASIERTER WETTBEWERB („FACILITIES BASED COMPETITION")}

Bei dem infrastrukturbasierten Wettbewerb handelt es sich um einen Markteintritt in den vorgelagerten Markt. Ein Newcomer verfügt uber eine eigene Netzinfrastruktur, über die er seine Telekommunikationsdienste anbieten oder aber Kapazitäten an andere Wettbewerber im nachgelagerten Markt vermieten kann. Unter

Vgl. Distelkamp (1999), S. 15.

Auf die Entbündelung von Netzelementen wird in Kapitel 2.4.2.2 năher eingegangen. Auf welche Art und Weise Entgelte für die einzeln anmietbaren Netzelemente festgelegt werden können und wie sie tatsăchlich bestimmt werden, wird in Kap. 5.1, 5.2 und 5.4 beschrieben. 
dem Ausgangspunkt, daß der Incumbent im vorgelagerten Markt der einzige Anbieter an Netzinfrastruktur sowohl im Weitverkehrsbereich als auch im Teilnehmeranschlußbereich ist, hat ein Markteintritt eines zweiten Netzinfrastrukturanbieters zur Folge, daß zu einem großen Teil Netzinfrastruktur doppelt verlegt würde.

Im Fernnetzbereich bzw. in dicht besiedelten Regionen wird die nachgefragte Kapazität groß genug sein, besonders im Zusammenhang mit erweiterten Mehrwertdiensten, die zunehmend nachgefragt werden, so daß eine doppelte Verlegung wünschenswert sein kann. ${ }^{7}$

Im Teilnehmeranschlußbereich ist der Bypass schon länger als alternativer Anschluß bekannt. Es handelt sich hierbei um eine selektive Anbindung von Geschäftskunden mit einer hochvolumigen Nachfrage, deren Kommunikationsdienste von einem alternativen Netzbetreiber unmittelbar in den Weitverkehrsbereich weitervermittelt werden.

In Teilnehmeranschlußbereichen von dünnbesiedelten Regionen besteht das Problem, daß für eine doppelte Verkabelung die Nachfrage zu gering ist und Skalenerträge nur unzureichend ausgeschöpft werden können. In diesem Zusammenhang werden oft die Argumente gegen eine Doppelverlegung, die sich aus der Existenz eines natürlichen Monopols ableiten, genannt. ${ }^{8}$ Aus diesem Grund wird der Teilnehmeranschlußbereich auch häufig als Bottleneck-Bereich bezeichnet. Ein erfolgreicher Markteintritt in den Markt für Netzinfrastruktur im Bottlenck-Bereich unterliegt besonderen Herausforderungen. Ein alternativer Carrier trägt erstens ein sehr hohes Erfolgsrisiko aufgrund der zu leistenden hohen irreversiblen Investitionskosten in eine Netzinfrastruktur. Zweitens gibt es für den Konsumenten selbst bei geringeren Preisen Anreize, nicht zu dem neuen Anbieter zu wechseln, weil für ihn durch den Wechsel monetäre wie nicht-monetäre Kosten entstünden.

Für die weitere Betrachtung ist es von Bedeutung, auf welche Technologien die Newcomer für die Etablierung einer alternativen Kommunikationsnetzinfrastruktur zurückgreifen. Ein Aufbau eines Netzes mit den identischen Eigenschaften des schon bestehenden Netzes ist in den Bottleneck-Bereichen ökonomisch nicht sinnvoll. Ursachen dafür sind eine zu geringe Nachfrage und das Bestehen von Kostensubadditivität. Der Aufbau einer alternativen Kommunikationsinfrastruktur im Bottleneck-Bereich ist aber dann denkbar, wenn sie erstens eine Produktvielfalt bzw. eine produktmäßige Erweiterung oder eine Qualitätssteigerung der bisherigen Basisdienste ermöglicht. ${ }^{9}$ Hier sei die produktmäßige Erweiterung in Form von Breitbanddiensten genannt. Deren Angebot setzt teilweise neues Verle-

Vgl. dazu Kap. 2.3.2, 2.3.3, 2.3.4.

Vgl. dazu Kap. 2.3.1.

Vgl. dazu Kap. 2.3.3, 2.3.4. 
gen von Infrastruktur voraus (z.B. Glasfaser). Als Beispiel für Produktvielfalt aufgrund kombinierter Produkte kann auf die zunehmende Konvergenz der Märkte Medien, Internet und Information hingewiesen werden. ${ }^{10}$ Zweitens ist der Aufbau einer alternativen Netzinfrastruktur denkbar, wenn sie auf neue Technologien zurückgreift, deren Einsatz keine vergleichbar hohen Investitionskosten erfordert. Hier sei besonders auf Anschlußtechnologien verwiesen, für die keine Anschlüsse neu verlegt werden müssen. Stattdessen können bestehende Kabelanschlüsse oder Röhren zusätzlich für Kommunikationsdienste genutzt werden. In Frage kämen die Leitungen des Kabelfernsehens (CA-TV) oder die Aufrüstung von Stromleitungen mit Hilfe von Powerline Communication Systemen (PLC). Diese Alternativen sind dann von besonderem Interesse, wenn die Umrüstungskosten der bestehenden Netzinfrastruktur unter den Ausbaukosten eines neuen Netzes liegen." Der Einsatz kabelloser Infrastruktur wie den Wireless Local Loop (WLL) zeichnet sich durch besonders niedrige Investitionskosten im Teilnehmeranschlußbereich im Vergleich zum herkömmlichen Teilnehmeranschluß aus. ${ }^{12}$ Folglich läßt sich eine Unterscheidung der Newcomer in eine Gruppe machen, die erst ein vollständig neues Netz im Anschlußbereich aufbauen müssen (WLL) und eine, die bestehende Netzinfrastruktur umrüsten (PLC und CA-TV).

Wenn eine produktmäßige Erweiterung und die Benutzung alternativer Technologien in Betracht gezogen werden, ist ein Markteintritt in den vorgelagerten Markt in den Bottleneck-Bereichen durchaus denkbar. Es könnte sich ein Wettbewerb im Teilnehmeranschlußbereich ergeben, der durch substitutiven Wettbewerb gekennzeichnet ist. Das zentrale Element substitutiven Wettbewerbs ist, daß die Produkte, die sich sehr ähnlich sind, aber nicht identisch sein müssen, zueinander im Wettbewerb stehen. Für den Teilnehmeranschlußbereich bedeutet das, daß ein Telefonteilnehmer zwischen mindestens zwei Anschlüssen, die von unterschiedlichen Netzbetreibern angeboten werden, wählen kann. Die Existenz von Substitutionswettbewerb schränkt die Marktmacht und den Preissetzungsspielraum eines Incumbents ein. ${ }^{13}$ Nimmt der Incumbent aufgrund seiner Marktmacht einen ,zu hohen' Preis für die Bereitstellung von Telekommunikationsdiensten, wird aufgrund der hohen Preise für alternative Carrier ein Anreiz geschaffen, Substitute zu entwickeln. Anbieter von Substituten, die bislang noch nicht zum relevanten Markt zählten, versuchen, durch Preis - und Produktvariationen bzw. produktmäBige Erweiterungen in den relevanten Markt einzutreten. Bei ,zu hohen Preisen“ wird ebenso der Konsument langfristig versuchen, auf ein Substitut umzustei-

\footnotetext{
Vgl. Kap. 2.1.1.3.

Weitere Details vgl. dazu Kap. 3.4.1.

Vgl. auch 3.4.2.

Vgl. Schmidt (1996), S. 66.
} 
gen. ${ }^{14}$ Substitutionswettbewerb entfaltet seine Wirksamkeit erst in der langen Frist. Mit Zunahme des technischen Fortschritts nimmt die Bedeutung des Substitutionswettbewerbs zu, weil er die Möglichkeiten der Entwicklung substitutiver Produkte erhöht. ${ }^{15}$

Die Vorteile von infrastrukturbasiertem Wettbewerb sind, daß Wettbewerber nicht mehr von dem einzigen Anbieter auf dem vorgelagerten Markt abhängig sind, der als Incumbent zudem noch auf dem nachgelagerten Markt ist und dort die Marktmacht hat. Anstelle eines ,One-Way-Access' würde also ein ,Two-WayAccess' treten. Die Nachteile infrastrukturbasierten Wettbewerbs bestehen darin, daß bei identischer Technologie soziale Kosten verursacht werden: Weil die Infrastruktur dupliziert wird, können ,Economies of Scale' sowie Dichte- und Verbundvorteile nicht mehr effizient ausgeschöpft werden. Setzt man aber unterschiedliche Technologien mit jeweils anderen Kostenstrukturen voraus, die zudem substitutive Produkte anbieten, die die Produktvielfalt und -qualität erhöhen, ist dieses Argument nicht mehr ausschlaggebend.

Für effektiven Wettbewerb muß neben der Zusammenschaltung, die die Kompatibilität beider Netzstrukturen gewährleistet, Nummernportabilität vorausgesetzt werden können, so daß das Wechseln als Kunde von einer zu anderen Gesellschaft erleichtert wird. ${ }^{16}$

Zusammenfassend läßt sich festhalten, daß der infrastrukturbasierte Netzwettbewerb zwar die teuerste Option fur den Entrant ist. Sie ist aber langfristig die Variante, die den Wettbewerb nachhaltig fördert, weil der Incumbent im Vergleich zu den anderen Markteintrittsformen zunehmend mit dem Ausbau alternativer Netze an Einflußnahme auf den Newcomer verliert. Im Folgenden soll eine sachlich relevante Marktabgrenzung vorgenommen werden, um infrastrukturbasierte Wettbewerbsverhältnisse besser beurteilen zu können. Vorab wird das angewendete Konzept zur Marktabgrenzung vorgestellt.

\footnotetext{
14 Im Rahmen der Analyse um Marktabgrenzung im Telekommunikationssektor wird auf Möglichkeiten und Bedeutung von Nachfrage- und Angebotssubstitution explizit eingegangen werden. Vgl. dazu Kap. 3.2 und 3.3.

Als Marktform kann sich vermutlich ein enges Oligopol ergeben, das die Gefahr aufeinander abgestimmten Verhaltens oder bewußten Parallelverhaltens und der Kollusion mit sich bringen kann. Vgl. Monopolkommission (1996) Tz. 59; Diestelkamp (1999) S. 12. Eine detaillierte Analyse dazu vgl. in Kap. 5.3.

Vgl. dazu Kap. 2.4.3, 2.4.5.
} 


\subsection{KRITERIEN DER MARKTABGRENZUNG UND MARKTSTRUKTUR}

Eine sinnvolle Marktabgrenzung ist notwendig, um den relevanten Markt bestimmen zu können. Mit Hilfe der Marktabgrenzung soll die Frage beantwortet werden können, inwieweit auf einem Markt wirksamer Wettbewerb vorliegt oder inwieweit er eingeschränkt ist bzw. Marktbeherrschung vorliegt. ${ }^{17}$

\subsubsection{DER VERGLEICHSMARKTANSATZ}

Vergleichsmarktkonzepte sind im allgemeinen Wettbewerbsrecht für Märkte angelegt, in denen kein oder nur geringfugiger Wettbewerb herrscht. Vergleiche können in räumlicher, zeitlicher oder sachlicher Hinsicht vorgenommen werden. ${ }^{18}$ Hier soll nur das sachliche Vergleichsmarktkonzept kurz vorgestellt werden. Dieses zieht zum Vergleich des zu betrachteten Marktes wettbewerbliche Märkte mit gleichartigen aber nicht identischen Gütern heran, die durchaus auch im Ausland liegen können. ${ }^{19}$ Dabei sollten die Märkte, die zum Vergleich herangezogen werden, funktionsfähigen Wettbewerb ${ }^{20}$ vorweisen können.

Eine Marktabgrenzung nach dem sachlichen Vergleichsmarktkonzept kann anhand zweier Dimensionen vorgenommen werden. Zum einen gibt es die sachlich relevante Marktabgrenzung, die sich auf die Produkteigenschaften konzentriert. Die Bestimmung des sachlich relevanten Marktes kann nach einer horizontalen Abgrenzung, d.h. nach Produkten und Kundengruppen, oder nach einer vertikalen

17 Wirksamer Wettbewerb („Effective Competition“) will als dynamischer Prozeß verstanden werden, bei dem Marktunvollkommenheiten sowie monopolistische Elemente für die Wirksamkeit des Wettbewerbs erforderlich sind. Zu einer ausfuhrlichen Darstellung wirksamen Wettbewerbs vgl. Schmidt (1996), S. $10 \mathrm{ff}$.

18 Vgl. Schmidt (1996), S. 269 ff.

19 Vgl. Herdzina (1999), S. 57. In $\S 3$ Abs. 3 TentgV ist geregelt, daß die Regulierungsbehörde Preise und Kosten von Unternehmen, die entsprechende Leistungen auf vergleichbaren Mărkten im Wettbewerb anbieten, als Vergleich herangezogen werden können. Vgl. Schütz (2001), S. 552 sowie Veröffentlichungskommission des OVG Münster (2001), S. 550. Bei der Liberalisierung des deutschen Telekommunikationsmarktes 1998 wurden mangels Kostenkenntnissen Zugangs- wie Zusammenschaltungsentgelte seitens der Regulierungsbehörde auf der Grundlage von internationalen Vergleichen festgelegt. Vgl. Mellewigt/Theissen (1998), S. 594 sowie Schütz (2001), S. 552.

20 Funktionsfahiger Wettbewerb unterscheidet sich vom vollkommenen Wettbewerb dahingehend, daß die Konkurrenz eine flexible Anpassung der betrieblichen Kapazităten an Schwankungen der Nachfrage und die dem technischen Fortschritt zugrunde liegende Schaffung neuen Wissens nur in weiten Oligopolen zu erfullen vermag. Vgl. Kantzenbach (1967) sowie Schmidt (1996), S. $11 \mathrm{ff}$. 
Abgrenzung, d.h. nach Produktionsstufen erfolgen. Zum anderen kann eine Abgrenzung anhand des geographisch relevanten Marktes vorgenommen werden. Hier wird eine Region hinsichtlich des relevanten Marktes abgegrenzt.

Um eine Marktabgrenzung nach sachlichen Vergleichsmarktkriterien vornehmen zu können, wird ein Konzept zur Abgrenzung von Produkten und Anbietern benötigt. Im folgenden Kapitel werden dazu verschiedene Substitutionskonzepte vorgestellt.

\subsubsection{SUBSTITUTIONSKONZEPTE ZUR SACHLICHEN MARKTABGRENZUNG}

Es kommen methodisch verschiedene Ansätze für die Bestimmung des für den Wettbewerbsprozeß relevanten Marktes in Frage. ${ }^{21}$ Bei einer Analyse einer technisch sehr dynamischen Branche, wie sie in der Telekommunikation vorliegt, die durch das Zusammenwachsen von Märkten aus unterschiedlichen technischen Bereichen (TIME-Märkte) geprägt ist, ist es erforderlich, daß Substitutionskonzepte zur sachlichen Marktabgrenzung herangezogen werden. Aus diesem Grund werden hier ausschließlich einige Substitutionskonzepte kurz vorgestellt.

Die relevante Marktbestimmung ist auf die klassische Definition eines Marktes in der Tradition von Cournot und Marshall zurückzuführen. Hier werden Märkte durch das Arbitrage-Prinzip abgegrenzt. Innerhalb des Marktes liegen einheitliche Preise vor und die betrachteten Güter sind perfekte Substitute. Das klassische Marktkonzept wurde erstmals mit Werken über monopolistischen Wettbewerb von Chamberlin und Robinson hinterfragt. ${ }^{22}$

Robinson befaßte sich im Rahmen ihrer Theorie der Substitutionslücke ${ }^{23}$ erstmals mit dem Problem einer relevanten Marktabgrenzung, wenn Produktdifferenzierung vorliegt und Substitutionsbeziehungen zwischen Gütern unvollständig sind. Das Konzept umfaßt eine Gruppe nicht-identischer Konsumgüter, die gute Substitute füreinander sind. Sie stehen in totaler Konkurrenz in bezug auf die Kaufkraft der Konsumenten zueinander. Die Substitute werden durch Lücken in der Substitutionskette zwischen der betreffenden Industrie und dem nächstbesten Substitut unterbrochen. Beispielsweise bestehen zwischen den Produkten Brot, Kartoffeln, Reis, Nudeln unterschiedlich starke Substitutionslücken, die zu eigenen relevanten Märkten führen. Die Substitutionslücke bezieht sich auf sachliche,

21 Einen allgemeinen Überblick über Marktabgrenzungskonzepte geben auch Klaue/ Schwintowski (2001), S. $24 \mathrm{ff}$.

22 Vgl. Robinson (1969), Chamberlin (1933).

23. Vgl. Robinson (1969). 
aber auch auf persönliche, räumliche und zeitliche Präferenzen seitens der Nachfrager. $^{24}$

In der Theorie der Marktbeziehungen nach Stackelberg ${ }^{25}$ ist der Ausgangspunkt das Bestehen vollkommener Elementarmärkte, die durch das Fehlen von sachlichen, persőnlichen, răumlichen und zeitlichen Prăferenzen gekennzeichnet sind (Homogenitătsbedingung). Ein Elementarmarkt ist der größte vollkommene Teilmarkt eines unvollkommenen Gesamtmarktes. Marktabgrenzungen lassen sich zwischen den Elementarmärkten ziehen, deren Substitutionsbeziehungen gering sind. Aufgrund von Substitutionsbeziehungen zwischen den vollkommenen Teilmärkten entsteht ein unvollkommener Gesamtmarkt.

Das Bedarfsmarktkonzept nach Amdt und Abbott und Bain ${ }^{26}$ grenzt den Markt nach Gütern ab, die einen bestimmten gesellschaftlichen Bedarf decken. Dabei handelt es sich bei den Gütern um enge Substitute, die dieselbe Kundenzielgruppe ansprechen (Bain). Man spricht auch von marktgleichwertigen Produkten, wenn deren Verwendungszweck vergleichbar und austauschbar ist und deren Preise ähnlich sind. Dieses Konzept ist zwar wissenschaftlich weniger exakt, dafür aber wettbewerbspolitisch handhabbarer. ${ }^{27}$

Das Konzept des verständigen Verbrauchers leitet sich aus dem Bedarfsmarktkonzept ab. Es geht hierbei um die funktionale Austauschbarkeit der fraglichen Produkte oder Dienste aus Sicht des verständigen Verbrauchers. Im Vordergrund steht die Reaktion eines einzelnen repräsentativen Verbrauchers. Dabei geht es um die zentrale Frage, welche Produkte der verständige Verbraucher als Substitute empfindet. Eine fiktive dauerhafte Erhöhung kleiner relativer Preise liefert die Grundlage für die Nachfragereaktion. ${ }^{28}$

Das Konzept der kombinierten Substitutions- und Produktionsflexibilităt von Kaysen und Turner bezieht neben der Nachfrage- auch die Angebotssubstitution in die Definition des relevanten Marktes ein. ${ }^{29}$ Neben der Austauschbarkeit von Produkten auf der Nachfragerseite soll hier ebenso Produktionsflexibilităt auf Seiten der Anbieter zur Marktabgrenzung herangezogen werden. Die Berulcksichtigung von Angebotssubstitution fand in bezug auf die Marktabgrenzung bislang weder in Deutschland noch auf EU-Ebene Anwendung, sondern erst in einem späteren Schritt im Rahmen der Feststellung von Marktbeherrschung. ${ }^{30}$

24 Vgl. Schmidt (1996), S. $49 \mathrm{ff}$.

25 Vgl. Stackelberg (1934), S. 29 ff.

26 Vgl. Arndt (1958), S. 217 ff., 224 und Abbott (1958), S. 96 und Bain (1968), S. 6 und 224.

27 Zur Beurteilung des Konzepts vgl. Klaue/Schwintowski (2001), S. 29 ff.

$28 \mathrm{Zu}$ einer ausfuhrlichen Beschreibung einer fiktiven Preiserhöhung vgl. Plum/SchwarzSchilling (2000), S. $16 \mathrm{ff}$.

29 Vgl. Kaysen/Turner (1959), S. 295.

30 Weitere Konzepte, die eine Marktabgrenzung aus einer Anbietersicht vornehmen, sind unter anderem das der externen Interdependenz [vgl. Triffin (1940)], bei dem Unternehmen, die 
Das Konzept des hypothetischen Monopolisten liefert die umfassenste Definition des relevanten Marktes, weil es neben den Möglichkeiten der Nachfrage- und Angebotssubstitution zusätzlich die Einflüsse potentiellen Wettbewerbs berücksichtigt. Im folgenden Kapitel soll dieses Konzept ausfuhrlicher vorgestellt und im weiteren Verlauf angewendet werden.

\subsubsection{DAS KONZEPT DES HYPOTHETISCHEN MONOPOLISTEN}

Das Konzept des hypothetischen Monopolisten liegt unter anderem den ,US Merger Guidelines' zugrunde und ist 1984 erstmals vom ,Department of Justice' in den ,Merger Guidlines' vorgestellt worden. Inzwischen wird das Konzept vielerorts diskutiert. Auch die Europäische Kommission sowie die englische Regulierungsbehörde ,Office of Telecommunications' (Oftel) arbeiten damit. ${ }^{31}$ Bei diesem Konzept handelt sich um eine analytische Vorgehensweise, und es ist nicht geeignet, Aussagen über reale Wettbewerbssituationen oder mögliche Preiserhöhungsspielräume zu machen. Ausgangspunkt sind Produkte oder Dienste eines Unternehmens, dessen Marktbeherrschung in Frage steht. Der Anbieter dieser Güter ist der hypothetische Monopolist. ${ }^{32}$ Das Konzept des hypothetischen Monopolisten untersucht nun Substitutionswirkungen infolge einer fiktiven dauerhaften Preiserhöhung von ca. 5-10\% ${ }^{33}$ seitens des Incumbents ebenso wie bei dem Konzept des verständigen Verbrauchers. Kommt es bedingt durch die Preiserhöhung

beim Verkauf voneinander abhängig sind, einem Markt zugeordnet werden oder das der Wirtschaftspläne [vgl. Schneider (1972), S. 68], das Unternehmen umfaßt, die in ihren Wirtschaftsplan die Entscheidungen anderer Unternehmen berücksichtigen, einem Markt zugehörig sind. [Vgl. Schmidt (1996). S. 49 ff.] Außerdem gibt es noch das Konzept der räumlichen Marktabgrenzung, das ein geographisches Gebiet definiert, in dem sich durch ungehinderten Handel Transportkosten angleichen. [Vgl. hierzu Kallfaß (1997)] Im Rahmen von zeitlichen Marktabgrenzungskonzepten können zeitliche Marktgrenzen gesetzlich, technisch oder natürlich bedingt sein. [Vgl. Schmidt (1996), S. 53]. Eine zeitliche Marktabgrenzung ist sinnvoll, wenn eine marktbeherrschende Stellung auf eine bestimmte Zeitspanne begrenzt ist. Vgl. Möschel (1992).

31 Vgl. Europäische Kommission (1997), Oftel (1998). Die deutschen Kartellbehörden wenden dagegen das Konzept des verstăndigen Verbrauchers an, das sich aus dem Bedarfsmarktkonzept ableitet. Dieses Konzept ist allerdings nicht so umfassend wie das hier verwendete, weil es nur die Möglichkeit der Substitution auf der Nachfrageseite beleuchtet. Mehr dazu vgl. Kuhlenkampff (2000), S. 53ff.; Plum/Schwarz-Schilling (2000), S. 15.

32 Die folgenden Ausfuhrungen zu dem Konzept des hypothetischen Monopolisten basieren auf Plum/Schwarz-Schilling (2000) sowie Stumpf/Schwarz-Schilling (1999).

33 Hierfür gibt es keine analytische Begründung. In der Praxis hat sich als Daumenregel bewährt, daß eine Preiserhöhung von $5-10 \%$ als ,small but siginificant ' anzusehen ist. Dabei ist zu beachten, daß je höher der Prozentsatz für eine Preiserhöhung ist, desto breiter die Definition des relevanten Marktes wird. Vgl. Oftel (1998), S. 18. Dies ist auch bekannt als SSNIPTest - Small but Significant and Non-transitory Increase In Prices. 
zu Substitutionsprozessen, kann dies als Begrenzung von Marktmacht bzw. als Indiz für Wettbewerb gesehen werden. Als relevanten Markt lassen sich demnach alle Anbieter von Produkten bezeichnen, die von dem Substitutionsprozess hinreichend berührt werden.

Ziel der Marktabgrenzung ist es, alle Produkte, die die Marktmacht eines hypothetischen Monopolisten beschränken können, in den relevanten Markt einzubeziehen, um die Wettbewerbssituation adäquat beurteilen zu können. Das Konzept des hypothetischen Monopolisten berücksichtigt hierbei neben den Möglichkeiten für Nachfrager, bei einer Preiserhöhung auf Substitute auszuweichen, auch die Substitutionsmöglichkeiten der Anbieter, indem Wettbewerber mit ihrem Angebot kurzfristig auf den Preisanstieg des Marktbeherrschers reagieren. Zudem berücksichtigt das Konzept die Möglichkeiten des potentiellen Wettbewerbs, das die Substitutionsbereitschaft der Anbieter aus einer längerfristigen Perspektive beobachtet. Dabei sollen die vorgestellten Kriterien den Umfang der Substitutionswirkungen festlegen.

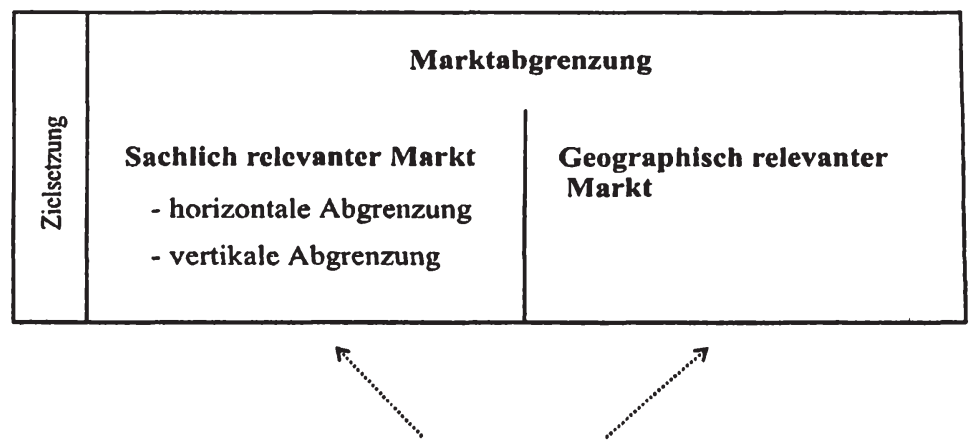

\begin{tabular}{|c|c|c|c|}
\hline E. & & es hypothetis & opolisten \\
\hline 总 & $\begin{array}{l}\text { Nachfrage- } \\
\text { substitution }\end{array}$ & $\begin{array}{l}\text { Angebots- } \\
\text { substitution }\end{array}$ & $\begin{array}{l}\text { Potentieller } \\
\text { Wettbewerb }\end{array}$ \\
\hline
\end{tabular}

Abbildung 3.2-1: Zielsetzung und Instrumentarium der Marktabgrenzung ${ }^{34}$

34 Vgl. Kuhlenkampff (2000), S. 53. 
Die Abbildung 3.2-1 soll graphisch veranschaulichen, daß mit Hilfe der Instrumentarien des Konzepts eine sachlich bzw. geographisch relevante Marktabgrenzung vorgenommen wird.

\subsubsection{NACHFRAGESUBSTITUTION}

Möglichkeiten der Nachfragesubstitution tiben vermutlich die stärkste disziplinierende Kraft auf den Wettbewerb aus. ${ }^{35}$ Zur Debatte steht, auf welche Produkte Nachfrager bei spürbarer Preiserhöhung ausweichen bzw. welche Produkte sie als substituierbar empfinden. In diesem Zusammenhang wird sich wiederum an dem in Kapitel 3.2.2 kurz vorgestellten Bedarfsmarktkonzept nach Arndt, Abbott und Bain orientiert. Dabei kommt es darauf an, daß zum einen eine Abwanderung an Nachfragern für den hypothetischen Monopolisten spürbar ist und zum anderen daß Nachfrager kurzfristig auf Preisveränderungen reagieren können. Die Wirksamkeit von Nachfragesubstitution kann durch staatliche Eingriffe wie regulatorische Barrieren beeinträchtigt werden sowie durch anfallende Kosten für zusätzliche Investitionen wie technische Umrüstungskosten oder Investitionen ins $\mathrm{Hu}$ mankapital. ${ }^{36}$

Es können verschiedene Methoden in Frage kommen, um das Ausmaß an Nachfragesubstitution feststellen zu können. Es gibt zum Beispiel die Möglichkeit, über Elastizitätsgrößen wie direkte Preiselastizitäten, Kreuzpreiselastizitäten oder Substitutionselastizitäten, nachfrageseitige Substitutionsbeziehungen zu messen. Das Zurückgreifen auf Elastizitäten bringt in der Regel Probleme bei der Ermittlung der notwendigen Preis- und Mengendaten mit sich, die für eindeutige Aussagen umfangreiche Datensätze benötigen. Außerdem sind Elastizitätsgrößen vergangenheitsorientiert und variieren im Zeitablauf aufgrund technischen Fortschritts. Eine weitere Möglichkeit wäre, Preisentwicklungen und die zugehörigen Nachfrageentwicklungen in der Vergangenheit zu analysieren. Sind zwei Produkte als enge Substitute anzusehen, so wird auch ihre Preisentwicklung ähnlich verlaufen. Auf der anderen Seite lassen parallele Preisentwicklungen nicht eindeutig darauf schließen, daß zwei Produkte dem gleichen relevanten Markt angehören. Befragung von Marktteilnehmern wäre eine weitere Möglichkeit, Substitutionsbeziehungen zu ermitteln. Die Umfragen müssen allerdings repräsentativ und unvoreingenommen sein.

35 Vgl. Europäische Kommission (1997), S. 3.

36 Bei Berücksichtigung von differenzierten Produkten ist die Entscheidung über Substitutionsmöglichkeiten schwieriger. Die Aussagekraft des Marktanteils für die Beurteilung der Wettbewerbsverhältnisse ist in Märkten mit starker Produktdifferenzierung geringer einzuschätzen als bei homogenen Produkten. Vgl. Baker/Wu (1998), S. 8. 


\subsubsection{ANGEBOTSSUBSTITUTION}

Bei der Angebotssubstitution geht es um Reaktionen anderer Anbieter auf signifikante Preiserhöhungen des hypothetischen Monopolisten, die ihn in seiner Preismacht disziplinieren. Die Anpassungsreaktion muß kurzfristig und ohne hohe Anpassungskosten erfolgen und groß genug sein, um Preiserhöhungen des Monopolisten unprofitabel zu machen.

Es kann zu Anpassungsreaktionen von Unternehmen im gleichen Markt in Form von Preis- und/oder Marketingstrategien kommen. Hierbei handelt es sich um Unternehmen, die gleichartige Produkte herstellen, die sich allenfalls in der Qualităt unterscheiden. Anpassungsreaktionen können ebenso von Unternehmen in angrenzenden Märkten erfolgen, also von Firmen, die verwandte Produkte herstellen und die ihr Angebot kurzfristig umstellen können durch Aktivierung von Reservekapazitäten oder Umwidmung von bereits genutzten Kapazitäten, ohne daß große Umstellungs- und Investitionskosten anfallen.

Zur Feststellung von Angebotssubstitution kommt wie bei der nachfrageseitigen Substitution die Befragung von Marktteilnehmern wie Wettbewerber, Anbieter von Vorprodukten und Vorleistungen und von Investitionsgutern in Frage. Eine andere Möglichkeit besteht darin, uber indirekte Beobachtungen zu ermitteln, in dem Reaktionen auf Preisveränderungen oder Änderungen anderer Wettbewerbsparameter analysiert werden.

Es gibt Differenzen, ob Angebotssubstitutionsmöglichkeiten bei der Abgrenzung des relevanten Marktes bei einer hypothetischen Preiserhöhung mit einbezogen werden sollen. Sowohl die EU als auch Oftel wollen sie berücksichtigen, wenn sie kurzfristig das Preissetzungsverhalten des hypothetischen Monopolisten einschränken. Die ,US Merger Guidelines' sowie die ,EU Guidelines' im Telekommunikationssektor und die Literatur und Rechtspraxis in Deutschland berlicksichtigen sie nicht. ${ }^{37}$ Sie berücksichtigen sie erst bei einem späteren Schritt, nämlich der Untersuchung von Marktanteilen und der Feststellung von Marktbeherrschung.

Soll das Konzept des hypothetischen Monopolisten konsequent angewendet werden, ist es sinnvoll, Möglichkeiten der Angebotssubstitution zu überprüfen, da sie die Marktmacht des Monopolisten kurzfristig disziplinieren können. ${ }^{38}$ 


\subsubsection{POTENTIELLER WETTBEWERB}

Potentieller Wettbewerb unterliegt dem Konzept bestreitbarer Märkte nach Baumol et al. (1988). Hierbei handelt es sich um Unternehmen, die noch nicht in gleichen oder angrenzenden Märkten tätig sind ${ }^{39}$. Potentieller Wettbewerb wird oft der Angebotssubstitution zugerechnet. Die ,US Merger Guidelines' unterscheiden in „uncommitted“ und „,committed entry“. Der „uncommitted entry“ als Markteintritt ohne signifikante Markteintritts- und Marktaustrittskosten innerhalb eines Jahres werden wie Angebotssubstitution berücksichtigt. Der „committed entry" ist ein Markteintritt, der erhebliche Investitionen erfordert. Er wird bei der Marktabgrenzung in den ,US Guidelines' nicht mit einbezogen. Nach dieser Definition ist eine disziplinierende Wirkung potentiellen Wettbewerbs per definitionem ausgeschlossen. Doch auch für den potentiellen Wettbewerb gilt wie für die Angebotssubstitution: Soll das Konzept des hypothetischen Monopolisten konsequent angewendet werden, sollte potentieller Wettbewerb bei der Bestimmung der Marktabgrenzung mit einbezogen werden, wenn der potentielle Wettbewerb zu einer Disziplinierung der Marktmacht des Monopolisten beiträgt.

Liegen weder Markteintritts- noch Marktaustrittsbarrieren vor, dann funktioniert potentieller Wettbewerb wie aktueller Wettbewerb unabhängig von Größenvorteilen jedweder Art. Dies gilt nach Lehrbuchbedingungen nur unter folgenden Annahmen: ${ }^{40}$

- Es muß vollkommener Marktzutritt gewährleistet sein, d.h. der Marktzutritt für einen neu in den Markt eintretenden Wettbewerber muß sofort möglich sein und muß gegebenenfalls die gesamte Nachfrage abdecken können. ${ }^{41}$

- Der Incumbent reagiert nicht auf den Markteintritt eines zusätzlichen Anbieters, d.h. er paßt seine Preise zunächst nicht an. Es wird unterstellt, daß der Incumbent langsamer reagiert als der neue Anbieter.

- Marktaustritt ist jederzeit möglich, d.h. Investitionen müssen jederzeit reversibel sein. Es dürfen bei Markteintritt keine versunkenen Kosten entstehen (Hitand Run- Marktzutritt ohne Kosten).

Das Bestehen von Markteintrittsbarrieren verhindert Wettbewerb und macht potentiellen Wettbewerb unglaubhaft. Hierzu gehören institutionelle Barrieren (legislative oder administrative Maßnahmen des Staates), strukturelle Barrieren wie irreversible Investitionskosten, die bei Markteintritt getätigt werden müssen, und Skalenvorteile in der Produktion sowie Lernkurveneffekte und strategische Markteintrittsbarrieren in Form von strategischem Verhalten des Incumbents

\footnotetext{
39 Vgl. Kantzenbach/Krüger (1990), S. 62 f.

40 Vgl. Finsinger (1991), S. 160.

41 Markentreue bzw. ,brand loyality“ wird hier nicht berücksichtigt.
} 
(Ausnutzen von first-mover-advantages), die den Markteintritt potentieller Wettbewerber erschweren.

Allerdings sollte hier angemerkt werden, daß das Modell perfekt bestreitbarer Märkte keiner wirklichkeitsnahen Abbildung realer Prozesse in Telekommunikationsmärkten entspricht. Man muß in beinahe jedem realen Markt von der Existenz versunkener Kosten ausgehen. Die Effizienzwirkungen potentieller Konkurrenz fallen bei der Existenz von Markteintrittsbarrieren nicht schlagartig weg. Aber sie sinken graduell mit zunehmenden Markteintrittsbarrieren. ${ }^{42}$

In der Praxis wird sich die Bestreitbarkeit des lokalen Telekommunikationsmarktes in bezug auf Substitutionsmöglichkeiten wie die mögliche Nutzung der Netzstruktur von Kabel-TV, Strom oder kabellose Anschlußtechnik erheblich verbessern, sofern diese zu vertretbaren Kosten umgerustet bzw. aufgebaut werden können. $^{43}$

\subsection{Die AbGRENZUNG DES RELEVANTEN MARKTES FÜR INFRASTRUKTURBASIERTEN WETTBEWERB}

Für eine sinnvolle Marktabgrenzung in bezug auf infrastrukturbasierten Wettbewerb ist es erforderlich, den Endkundenmarkt fur Festnetzdienste und die CarrierMärkte im Rahmen der Untersuchung voneinander zu unterscheiden. ${ }^{44}$ Der Endkundenmarkt für Festnetztelefonie umfaßt neben Basis- und Breitbanddiensten ebenso die Bereitstellung von Teilnehmeranschlüssen. $\mathrm{Zu}$ den Carrier-Märkten zählen Originierungs- (bzw. Zufuhrungs-) und Terminierungsleistungen im Festnetz sowie der Zugang zu Teilnehmeranschlußleitungen sowie Märkte für Mietleitungen, die in Kapitel 3.3.2 näher betrachtet werden.

\subsubsection{MARKT FÜR FESTNETZDIENSTE}

Bei den Festnetzdiensten bietet es sich an, nach Produktgruppen bzw. nach Produkteigenschaften $\mathrm{zu}$ unterscheiden. Es kann folgende horizontale sachlich relevante Marktabgrenzung vorgenommen werden und grob zwischen dem Markt für Basisdienste und für Mehrwertdienste unterschieden werden:

\footnotetext{
42 Vgl. Kruse/Haucap (2002), S. 94.

43 Distelkamp (1999), S. 15.

44 Vgl. Stumpf/Schwarz-Schilling (1999), S. 1.
} 
Basisdienste bzw. Schmalbanddienste $(64 \mathrm{kbit} / \mathrm{s})$ beinhalten im wesentlichen die Sprachübermittlung. Im Rahmen der reinen Sprachübertragung lassen sich folgende Märkte voneinander unterscheiden:

- Markt für Teilnehmeranschlüsse und Ortsgespräche

Da die Bereitstellung eines Teilnehmeranschlusses bisher noch ausschließlich in Kombination mit Ortsgesprächen einhergeht, werden Teilnehmeranschluß und Ortsgespräche als einheitlicher Markt abgegrenzt. ${ }^{45}$ Bei Markteintritt von Wettbewerbern im Teilnehmeranschlußbereich sollte diese Marktabgrenzung kritisch überdacht werden und stattdessen in zwei Märkte, nämlich jeweils für Teilnehmeranschlüsse und für Ortsgespräche aufgeteilt werden.

- Markt für Ferngespräche

- Markt für Auslandsgespräche

- Markt für Gespräche zu Mobilfunkteilnehmern.

Neben den reinen Sprachdiensten bzw. Basisdiensten unterscheidet man davon Mehrwertdienste bzw. Breitbanddienste, die neben der Sprachübertragung vielfältige Zusatzdienste umfassen. Hierbei lassen sich folgende Märkte unterscheiden:

- Markt für sprachbasierte Mehrwertdienste

Dieser umfaßt neben Sprachmehrwertdiensten (Angebot an technischen Plattformen für Sprachboxen, Audiotext und Rufnummernauskünfte) ebenso $\mathrm{Zu}$ satzleistungen, die dem Nachfrager zusätzliche Mobilität ermöglichen (z.B. persönliche Rufnummer $\left.{ }^{46}\right)$. $\mathrm{Zu}$ unterscheiden sind ebenso unterschiedliche Zahlungsmodi wie beispielsweise Freephone (0800), Shared Cost (0180) oder Premium Rate Diensten (0190 bzw. 0900), die aus Nachfragersicht allerdings nicht austauschbar sind. ${ }^{47}$

- Markt für datenbasierte Mehrwertdienste

$\mathrm{Zu}$ den Datenmehrwertdiensten zählen unter anderem Verbindungen zu Internet-Diensten, e-mail, Telefax, Onlinedatenbanken, Electronic Data Interchange.

- Markt für videobasierte Mehrwertdienste

Hierzu zählt sämtliche bildgestützte Kommunikation wie zum Beispiel Videokonferenzen.

\footnotetext{
Vgl. Stumpf/Schwarz-Schilling (1999), S. 3.

Vgl. hierzu Kap. 2.4.4.

Vgl. Plum/Schwarz-Schilling (2000), S. 52.
} 
Eine Abgrenzung nach Kundengruppen erscheint insbesondere bei dem Markt für Teilnehmeranschlüsse sinnvoll. Kundengruppen können in Geschäfts- und Privatkunden nach folgenden $\mathrm{Kriterien}^{48}$ unterschieden werden:

- nach der Anschlußart (analog, ISDN,...),

- nach dem Umsatz, den ein Kunde generiert,

- nach dem gewählten Tarifpaket.

Eine răumlich relevante Marktabgrenzung von Festnetzdiensten in Form von Regionalmärkten ist für Ortsgespräche wie für Teilnehmeranschlüsse sinnvoll. Für Fern-, Auslands- und Mobilgespräche sowie für Breitbanddienste ist eine regionale Abgrenzung nicht sinnvoll. Regelungen zur Verbindungsnetzbetreiberauswahl und zur Zusammenschaltung ermöglichen es allen Verbindungsnetzbetreibern unabhängig von ihrer geographischen Ausbreitung ihrer Netze, überall in Deutschland Basis- wie Breitbanddienste anzubieten.

\subsubsection{MARKTABGRENZUNG NACH DEM KONZEPT DES}

\section{HYPOTHETISCHEN MONOPOLISTEN FÜR SPRACHDIENSTE}

Im Zuge der hier vorgeschlagenen Marktabgrenzung soll das Konzept des hypothetischen Monopolisten auf Sprachtelefonie angewendet werden. Der traditionelle Anbieter auf beiden Märkten - der Incumbent DTAG- kann als hypothetischer Monopolist gesehen werden. Betrachtet werden Wirkungen einer dauerhaften Preiserhöhung von ca. 5-10\% des hypothetischen Monopolisten auf Nachfrager und Anbieter unter Beruicksichtigung von Substituten. Es sollen hier nur Substitute berlicksichtigt werden, deren Anbieter über eigene Netzinfrastruktur verfügen, um die Analyse überschaubar zu lassen. Dabei können die Übertragungsmedien der Sprach- bzw. Datenübermittlung ganz unterschiedlich sein. Für den Nachfrager sind Sprachtelefondienst, Datenkommunikation oder Fernsehbildübertragung nicht prinzipiell austauschbar, da sie unterschiedliche Bedürfnisse der Nachfrager befriedigen. ${ }^{49}$ Aber in technischer Hinsicht und damit aus Infrastrukturanbieteransicht spielt es bei dem Transport digitaler Signale keine Rolle mehr, ob diese am Ende in Sprache, Bilder oder Daten umgesetzt werden. Es wird alles in digitalisierten Bits gemessen und in homogene Einheiten (Pakete) versendet. Die technische Entwicklung führt dazu, daß Anbieter verschiedener Arten von Infrastruktur Sprache, Daten oder Bilder transportieren können. Neben alternativen

\footnotetext{
48 Vgl. Stumpf/Schwarz-Schilling (1999), S. 6.

49 Allerdings verschwimmen selbst hier die Grenzen zunehmend. So betrachten viele Nachfrager email oder SMS beim Mobilfunk als Substitut zur Sprachvermittlung.
} 
Netzbetreibern wie zum Beispiel City Carrier, die in den Markt eintreten, können Nachfrager den herkömmlichen Netzbetreiber ebenso durch Mobilfunk nach GSM Standard sowie in Zukunft durch UMTS oder durch neue Technologien wie TVKabel, PLC oder Internet-Telefonie substituieren. Eine Beschreibung der einzelnen Substitute sowie ihre Chancen als Substitute werden in Kap. 3.4 näher erläutert.

Bei den Marktabgrenzungskriterien, wie in Kapitel 3.2 beschrieben, ist zum einen die Substitutionsbereitschaft der Nachfrager von Bedeutung, die durch folgende Faktoren beeinflußt wird:

- zusätzlich notwendige Investitionen seitens des Kunden,

- zusätzlicher Aufwand bei Gesprächsinitiierung,

- Sprachqualität,

- Höhe der maximalen Datenübertragungsraten,

- maximal mögliche Übertragungsentfernung,

- erweiterte Leistungsmerkmale wie z.B. Breitbanddienste.

Eine Substitution seitens der Nachfrager erfolgt im Falle zusätzlicher notwendiger Investitionen sowie zusätzlichen Aufwandes bei der Gesprächsinitiierung oder einer schlechteren Sprachqualität erst dann, wenn der Preisvorteil die Einbußen kompensiert. Auf der anderen Seite können erweiterte Leistungsmerkmale einen Preisnachteil kompensieren bzw. die Substitutionsbereitschaft der Nachfrager erhöhen. Die Höhe der übertragbaren Datenraten spielt dann eine Rolle, wenn der Kunde neben reinen Sprachdiensten zusätzlich auf Breitband- oder Internetdienste zugreifen will. Die Nachfrager weisen unterschiedliche Präferenzen hinsichtlich der Faktoren auf.

Zum anderen spielt die Substitutionsbereitschaft der Anbieter eine Rolle, d.h. die Reaktionen der Wettbewerber auf Preiserhöhungen des Incumbents.

Im Rahmen potentiellen Wettbewerbs als drittes Kriterium zur Anwendung des Konzepts des hypothetischen Monopolisten kommen alle Carrier in Betracht, die bereits über hinreichende Infrastruktur verfuigen, da ein schneller Markteintritt möglich sein sollte.

\subsubsection{CARRIER-MÄRKTE}

Carrier-Märkte sind Märkte für den Zugang zu Einrichtungen, die zur Erbringung von Telekommunikationsdiensten für den Endnutzer erforderlich sind (vorgelagerter Markt). Die Zugangsmärkte sind stark durch regulatorische Eingriffe bestimmt. ${ }^{50}$ Bei der näheren Betrachtung von Carrier-Märkten wird unterschieden in

so Vgl. hierzu institutionelle Rahmenbedingungen in Kap. 2.2. 
Zusammenschaltungsleistungen (Kapitel 3.3.3.1), den entbündelten Zugang zu

Teilnehmeranschlußleitungen (Kap. 3.3.3.2) sowie Mietleitungen (Kap.3.3.3.3).

\subsubsection{ZUSAMMENSCHALTUNGSLEISTUNGEN VON FESTNETZANSCHLÜSSEN}

Im Rahmen der Analyse von Zusammenschaltungsleistungen ${ }^{51}$ bietet sich eine vertikale sachlich relevante Marktabgrenzung an. Sie erfolgt nach Produktionsstufen. Ein Basis- wie Mehrwertdienst läßt sich in folgende Produktionsstufen einordnen:

- die Originierung bzw. Zuführung von Diensten, d.h. einer Schaltung einer Verbindung vom rufenden Anschluß zu einem Netzübergabepunkt,

- die Terminierung, d.h. eine Verbindung von einem Netzübergabepunkt bis hin zum angewählten Telefonanschluß.

Dabei kann der Netzübergabepunkt auf unterschiedlichen Netzebenen liegen: ${ }^{52}$

- lokal: bei lokalen Originierungs- wie Terminierungsleistungen liegt der Netzübergabepunkt bei einer OVSt. Die Zusammenschaltung ermöglicht den Zugang zu allen Teilnehmern im Bereich dieser Vermittlungsstelle.

- regional: Bei regionalen Originierungs- und Terminierungsleistungen liegt der Netzübergabepunkt bei einer WVSt. Dies ermöglicht den Zugang zu allen Teilnehmern in diesem Großraum.

- national: Hier liegt der Netzubergabepunkt ebenfalls bei einer WVSt. Er ermöglicht den landesweiten Zugang zu allen Teilnehmern des Betreibers.

Im Teilnehmeranschlußbereich ist die Originierungs- und Terminierungsleistung unverzichtbar. Darum werden für jeden Anbieter im Anschlußbereich zwei Märkte definiert: der Markt für lokale Originierung und der Markt für lokale Terminierung. Dabei ergeben sich so viele Märkte wie Netzbetreiber im Teilnehmeranschlußbereich. Die Regulierungsbehörde genehmigt Entgelte für Zusammenschaltungsleistungen (ex ante Regulierung der Zusammenschaltungsentgelte) der $D T A G$ und damit auch der anderen Netzbetreiber. ${ }^{53}$ Es bestehen indirekte $\mathrm{Zu}$ sammenhänge zwischen dem Endkundenmarkt fur Teilnehmeranschlüsse und den Carriermärkten für lokale Zuführung und Terminierung.

Die răumliche Marktabgrenzung geht einher mit der sachlichen. In dem Markt für lokale Originierungsleistungen und Teilnehmeranschlüsse sowie dem Markt für lokale Terminierungsleistungen und Teilnehmeran-

Zu der Vorteilhaftigkeit von Zusammenschaltung vgl. hierzu Kap. 5.

Vgl. Plum/Schwarz-Schilling (2000), S. 60.

Vgl. dazu Kap. 2.4.3. 
schlüsse sind Netzbetreiber Anbieter von lokalen Zuführungs- und Terminierungsdiensten. ${ }^{54}$ Dabei wird bislang der überwiegende Teil an Diensten von dem Incumbent erbracht. ${ }^{55}$ Jeder Anbieter dieser Leistungen ist Alleinanbieter. Eine kurzfristige Substitution ist damit nicht möglich. Mittel- bis langfristig können Endkunden ihren Anschlußbetreiber wechseln, wenn Preselection und Call by Call - Dienste aufgrund erhöhter Terminierungsentgelte seitens des Anschlußbetreibers sich für sie erhöhen sollte.

In dem Markt für regionale/nationale Originierung und Terminierung ist bislang noch der Incumbent der dominante Anbieter. Es ist allerdings ein Anstieg der Wettbewerbsintensität zu verzeichnen. ${ }^{56}$

Substitution von Zusammenschaltungsleistungen ist durch Netzwerweiterung in Form von Eigenproduktion von Netzleistungen denkbar. Dann muß ein Netzbetreiber keine nationale Originierungs- wie Terminierungsleistung vom Incumbent beziehen. Er kauft dann nur noch regionale und lokale Leistungen ein. Erfolgt eine Netzverdichtung auch auf der regionalen Ebene, dann müssen dementsprechend nicht mehr regionale Leistungen eingekauft werden. Lokale Leistungen kann nur der Betreiber von Teilnehmeranschlüssen anbieten. Eine Netzerweiterung ist ebenso in Form von Kooperationen zwischen Betreibern denkbar, zum Beispiel einen Verbund von City-Carrier. ${ }^{57}$

\subsubsection{ZUGANG ZU TEILNEHMERANSCHLUbLEITUNGEN}

Aufgrund der marktbeherrschenden Stellung der DTAG ist nur sie zur Vermietung von Teilnehmeranschlüssen an andere Netzbetreiber verpflichtet. ${ }^{58}$ Bisher verfügen andere Betreiber nur in geringem Umfang über eigene Teilnehmeranschlüsse und müssen diese auch nicht vermieten. Die Preise für die Miete der entbündelten Teilnehmeranschlußleitung sind von der RegTP festgelegt und orientieren sich an kostenorientierten Durchschnittswerten (Total Element Long Run Incremental

\footnotetext{
54 Bis Oktober 2000 gab es 31 Unternehmen, die als Netzbetreiber im Teilnehmeranschlußbereich agierten. Vgl. RegTP (2001a), S. 14. Vgl. hierzu Kap. 3.3.2.2.

56 Bis Mitte 2001 gab es 117 Zusammenschaltungsverträge zwischen der DTAG und anderen Netzebtreibern in Deutschland. Der Umsatz im Carrier-Geschäft ist von 1998 bis 2000 von 3,5 Mrd. DM auf 10,3 Mrd DM angewachsen. Davon gehen $53 \%$ an die DTAG, 13\% an Festnetzwettbewerber und 34\% an Mobilfunkbetreiber. Vgl. RegTP (2001b), S. 190 f.

57 Der Zusammenschluß vieler regionaler Netzbetreiber in RegioNet ermöglicht die Bereitstellung eines flächendeckenen Netzes. Beteiligt daran sind unter anderem EWETel, Hansenet; isis MultimediaNetz; NetCologne; tesion, VEW TELNET. 1998 umspannte die Zusammenschaltung der Netze eine Fläche von $35.000 \mathrm{~km}$. Vgl. Pressemitteilung der Regio Net $A G$ vom 16.09. 1998.

58 Vgl. hierzu Kap. 2.4.2.2.
} 
Cost (TELRIC)- Konzept) ${ }^{59}$. Bis Mitte 2001 basierten von Wettbewerbern eingerichtete Kanäle zu über $80 \%$ auf angemieteten Teilnehmeranschlußleitungen der DTAG. Die Tendenz ist steigend. ${ }^{60}$

\subsubsection{MIETLEITUNGEN FÜR NETZBETREIBER}

Mietleitungen sind Übertragungswege, die zwei Anschlußstellen dauerhaft miteinander verbinden. Jeder Inhaber einer Übertragungswegelizenz der Klasse 3 kann Mietleitungen anbieten und wird nur dem Mieter zur Nutzung überlassen.

Im Zuge einer horizontalen sachlich relevanten Marktabgrenzung können folgende relevante Märkte unterschieden werden ${ }^{61}$, nämlich der Markt für

- lokale Mietleitungen,

- Fernmietleitungen,

- internationale Mietleitungen.

Eine răumliche Abgrenzung geht einher mit der sachlich relevanten. Man unterscheidet

- Regionalmärkte für lokale Mietleitungen und

- bundesweite Märkte für Fernmietleitungen.

Bei einer Abgrenzung nach Kundengruppen ist zu erwähnen, daß Mietleitungen zum einen von Netzbetreibern in Anspruch genommen werden, zum anderen von Geschäftskunden (Bypass). Diese beiden Kundengruppen gilt es voneinander abzugrenzen, da es sich hierbei auch um unterschiedliche Märkte handelt.

Bei einer differenzierten Betrachtung der digitalen Segmente ist festzustellen, daß bis $155 \mathrm{Mbit} / \mathrm{s}$ die $D T A G$ nach wie vor der größte Anbieter trotz Umsatzrückgang der letzten Jahre ist. In dem Segment $>155 \mathrm{Mbit} / \mathrm{s}$ generiert die DTAG allerdings keine Umsätze mehr. Hier gibt es eine Vielzahl von Anbietern; die 10 größten Wettbewerber haben $70 \%$ Erlösanteil. Es herrscht dort reger Wettbewerb. ${ }^{62}$

\footnotetext{
59 Vgl. hierzu Kap. 5.4

60 Vgl. RegTP (2001b), S.174. Zur Entwicklung der Verträge über den Zugang zu Teilnehmeranschlußleitungen der $D T A G$ vgl. RegTP (2001a) S. $24 \mathrm{f}$.

Vgl. Stumpf/Schwarz-Schilling (1999), S. 66 ff.

Vgl. RegTP (2001a), S. 206.
} 
Seit der Liberalisierung 1998 hat sich der infrastrukturbasierte Wettbewerb im Teilnehmeranschlußbereich eher zurückhaltend entwickelt. Man kann immer noch von einer dominanten Marktmachtstellung des ehemals öffentlichen Netzbetreibers ausgehen. ${ }^{63}$ Dies kann folgende Ursachen haben:

- Es handelt sich unter anderem aufgrund hoher irreversibler Investitionskosten und des Vorliegens von Kostensubadditivität um ein natürliches Monopol im Teilnehmeranschlußbereich. ${ }^{64}$

- Außerdem sind alternative Anschlußtechnologien noch nicht marktreif.

- Die deutsche Regulierungsbehörde hat mit ihren regulatorischen Vorgaben den Dienstewettbewerb bzw. den Wettbewerb auf Grundlage angemieteter Teilnehmeranschlußleitungen sehr attraktiv gemacht, so daß Investitionsanreize in infrastrukturbasierten Wettbewerb im Teilnehmeranschlußberich reduziert oder zumindest verzerrt worden sind. ${ }^{65}$

Dabei muß bedacht werden, daß der Erfolg infrastrukturbasierten Wettbewerbs erst langfristig auszumachen ist, da der erforderliche Netzaufbau viel Zeit in Anspruch nimmt, insbesondere im Teilnehmeranschlußbereich. Und dort ist er auch am teuersten.

Inzwischen haben mehr als ein Viertel der Gesamtbevölkerung die Möglichkeit, einen Teilnehmeranschluß bei einem Wettbewerber zu beziehen. ${ }^{66}$ Auffallend sind dabei die starken Schwankungen bei Festnetzanschlußsegmenten. Beispielsweise sind die Marktanteile der Wettbewerber bei analogen Anschlüssen -also im Privatkundensegment- verschwindend gering $(0,6 \%)$, bei ISDN Basisanschlüssen 3,3\% und im Geschäftskundensegment bei Primärmultiplexeinrichtungen (PMX) $9,8 \%$ im 1.Quartal 2001. Daraus leitet sich für Geschäftskunden eine zunehmende Substitutionsbereitschaft ab. In Privatkundenmärkten muß man dagegen eine geringe Substitutionsbereitschaft verzeichnen. Dabei muß vor allem darauf hingewiesen werden, daß die Wettbewerbsentwicklung von der Bevölkerungsdichte einer Region abhängt. ${ }^{67}$ In Großstädten konnten bis zu 50\% der Bevölkerung zwi-

63 Bei den Märkten für Teilnehmeranschlüsse und Ortsgespräche hat die DTAG bundesweit einen Marktanteil von Festnetzanschlüssen von $97,9 \%$ nach vier Jahren der Liberalisierung des Marktes. Wettbewerber konnten für 2001 einen Marktanteil von 3\% ausmachen. Vgl. RegTP (2001b), S. 171.

64 Der Teilnehmeranschlußbereich wird hinsichtlich dieser Fragestellung in Kap. 2.3 besonders analysiert.

65 Vgl. Kap. 5 und 6.

66 Allerdings beinhaltet dies auch entbündelte angemietete Teilnehmeranschlußleitungen. Vgl. RegTP (2000), S. 15.

67 In Ballungsregionen wie in Köln konnten Anschlußwettbewerber bis Ende 2000 16\% Marktanteil vorweisen, in Düsseldorf 12\% und in Frankfurt a.M. 4\%. Vgl. RegTP (2001b), S. 171. 
schen einem Anschluß der DTAG oder eines Wettbewerbers wählen. Wahlmöglichkeiten bestand also in den Gebieten, in denen 1/3 der Gesamtbevőlkerung lebt. ${ }^{68}$ In der Peripherie ist kaum eine Wettbewerbsentwicklung zu beobachten.

Die Preisentwicklung ist wegen des geringen Wettbewerbs unverändert. Dies liegt allerdings auch daran, daß der Incumbent gemäß der Universaldienstverpflichtung keine Preisdifferenzierung hinsichtlich der Teilnehmeranschlüsse von Ballungsregionen und Peripherien vornehmen darf. ${ }^{69}$ Dabei ist der Marktzutritt im lokalen Bereich von der Umsatzhöhe her attraktiv. 50\% des Umsatzes wird derzeit in den Ortsnetzen erzielt ${ }^{70}$.

Mit Hilfe neuer Technologien kann der infrastrukturbasierte Wettbewerb eine neue Dimension erhalten, nämlich dann, wenn bestehende Netzinfrastruktur zusätzlich für Kommunikationsdienste genutzt werden kann oder wenn auf funkgestützte Technologien zurückgegriffen wird, dessen Netzaufbau schneller und kostengünstiger geht als ein kabelbasierter Netzaufbau. Mögliche Alternativen zur herkömmlichen Festnetztechnologie sind nämlich neben den Fernsehkabelnetzen und der Powerline Communication über Stromkabel der Richtfunk (Wireless Local Loop) und der Mobilfunk als funkgestützte Anschlußtechnologien. Sie alle haben den Vorteil, daß keine Neuverlegung von Festnetzen im Erdreich im Teilnehmeranschlußbereich notwendig ist. Die Monopolkommission sieht in diesen Technologien mittel- bis längerfristig ernstzunehmenden Wettbewerb. ${ }^{71}$

Bei den wettbewerblichen Anbietern handelt es sich weitestgehend um City Carrier. City Carrier wie Netcologne, ISIS oder Hansenet verfügen über eine regional begrenzte Netzinfrastruktur. Sie werden in der Regel von kommunalen Gesellschaften wie stådtischen Gas-, Strom- und Wasserwerken betrieben und bieten Schmal- und Breitbanddienste sowie Teilnehmeranschlüsse im privaten wie im Geschäftskundenbereich. Der infrastrukturelle Ausbau erfolgt zunächst vorrangig in Ballungsgebieten, d.h. bei hoher Nachfragedichte, weil die H8he der Verbundvorteile entscheidend ist. Inzwischen gibt es diverse regionale Betreiber, die in Ballungszentren lokal begrenzt Telekommunikationsdienste anbieten. Vgl. hierzu FAZ v. 11.5.2000.

In 83 Stădten mit mehr als 1000000 Einwohnern lag der Anteil bei $52 \%$; in 188 Städten mit über 50000 Einwohnern lag der Anteil bei 47\%. Vgl. RegTP (2001b), S. 172. Ein ausführlicher Städtevergleich findet sich in RegTP (2001a) S. 44 ff., ein internationaler Vergleich vgl. S. $50 \mathrm{ff}$.

69 Vgl. zur Universaldienstverpflichtung Kap. 2.4.5.

70 Vgl. Neumann (1999), S. 22.

71 Vgl. Monopolkommission (1999), RN 72. 


\subsubsection{NETZBASIERENDE ANSCHLUBTECHNOLOGIEN}

\subsubsection{DAS TV-BREITBANDKABEL (CA-TV)}

Deutschland hat eine der höchsten TV - Kabelanschlußdichten in Europa. 2001 empfingen ca. $60 \%$ aller Fernsehhaushalte TV über Breitbandkabel. ${ }^{72}$ Aufgrund einer hohen Verbreitung stellt es eine viel versprechende Alternative zu herkömmlichen Teilnehmeranschlußnetzen dar.

TV-Kabelnetze bestehen in der Regel aus einer Kopfstation, die Daten über eine Antenne empfängt, und dem Verteilnetz, das zur Übertragung hoch kapazitärer Dienste, zum Beispiel von Bilddaten, ausgelegt ist. Für das Verteilnetz wird Koaxialkabel zur Verteilung breitbandiger Rundfunkdienste $(47-450 \mathrm{MHz})$ hin zum Teilnehmeranschluß verwendet. Das Netz wird in vier Ebenen unterteilt: Auf der Netzebene 1 findet die Produktion der TV- und Hörfunksignale statt. Auf der Netzebene 2 werden die Signale vom Produktionsort zum terrestrischen Sender übertragen. Die Netzebene 3 stellt das lokale Verbindungsnetz dar. Hier beginnt das Verteilnetz. Die Signale werden von der Empfangsstelle weitergeleitet bis hin zum Übergabepunkt. Ab dem Übergabepunkt beginnt die Netzebene 4, die das sogenannte Hausanschlußnetz umfaßt. Die Ebenen 3 und 4 sind relevant für den Teilnehmeranschlußbereich für die Überbrückung der Letzten Meile. Der größte Teil des Kabelfernsehnetzes auf der Ebene 3 befindet sich derzeit weitestgehend im Besitz des Incumbents, ${ }^{73}$ so daß in den meisten Regionen faktisch ein Monopol vorliegt. Der direkte Teilnehmerzugang auf Netzebene 4 wurde überwiegend von Privatfirmen aufgebaut, d.h. 2/3 aller Endkunden werden von privaten Kabelnetzbetreibern betreut. ${ }^{74} \mathrm{Da}$ es sich um ein Verteilnetz handelt, ist es nur sehr begrenzt rückkanalfähig. Es gibt zwar eine Upstream-Kapazität, die bisher lediglich für Störungsmeldungen und Netzwerkmanagement genutzt worden ist. Dieser Teil muß aufgerüstet werden, um das Netz als ein Kommunikationsnetz - nämlich bidirektional - nutzen zu können, d.h. Umrüstungskosten fallen je nach Alter der Kabel unterschiedlich hoch an. ${ }^{75}$

Es gibt zwei Möglichkeiten der Erweiterung des Rückkanals: erstens über die Einrichtung eines Rückkanals auf dem Koax-Kabel (telephony over coax), d.h. ein

72 Von 36,13 Mio. Fernsehhaushalten nutzen ca. 60\% Zuschauer das Breitbandkabel, 30\% Satellit. Vgl. RegTP (2001b), S. $197 \mathrm{f}$.

73 Und zwar im Besitz der Kabel Deutschland GmbH (KDG), eine 100\% Tochterfirma der DTAG.

74 Vgl. Berthes, u.a. (1998), S. 25. KDG hat zu 1/3 aller Kabelfernsehhaushalte einen direkten Kundenzugang (Netzebene 4). Vgl. RegTP (2001b), S.197 f.

75 Die Herstellung eines unidirektionalen Zugangs zum Beispiel zum Internet ist mit Einsatz von Kabelmodems auf der Nutzerseite möglich. Vgl. Wickert-Nick (1999), S. 35. 
bestimmter Frequenzbereich wird für den bidirektionalen Verkehr reserviert. ${ }^{76}$ ,Telephony over coax ${ }^{6}$ hat den Vorteil, daß keine Neuverkabelung notwendig ist. Die Nachteile bestehen allerdings darin, daß notwendige Verstărker leicht störanfällig sind, weil sie eine unterbrechungsfreie Stromversorgung benötigen. Je mehr Verstärker notwendig sind, desto höher ist deshalb die Ausfallwahrscheinlichkeit. ${ }^{77}$ Ein weiterer Nachteil ist, daß es ,upstream' einen Übertragungsengpaß gibt (downstream $30 \mathrm{Mbit}$, upstream $4 \mathrm{Mbit}$ ). Außerdem kann es zu Störungen der Flugnavigation und anderer Funkdienste kommen. ${ }^{78}$

Die zweite Möglichkeit ist der overlay-approach, d.h. das Verlegen eines zusätzlichen Kupferdoppeladersystems für den bidirektionalen Verkehr. Dies würde den Vorteil mit sich bringen, daß bewährte Technologien eingesetzt werden. Doch dagegen sprechen Investitionskosten für das Verlegen zusätzlicher Kabel. Allerdings könnte das vorhandene Röhrensystem genutzt werden.

\subsubsection{DATENÜBERTRAgUNG ÜBER STROMNETZE}

Spätestens seit der Liberalisierung des Strommarktes ist das Interesse der Elektrizitätsversorgungsunternehmen (EVUs), neben Strom weitere Dienstleistungen bereitzustellen und in neue Märkte einzusteigen, zum Beispiel in Telekommunikationsmärkte mit Hilfe der sogenannten Powerline-Technologie. Unter dem Begriff Power Line Communication (PLC) subsumieren sich die verschiedenen Verfahren zur Datenübertragung über Strominfrastruktur.

Die Infrastruktur des Stromnetzes besteht aus mehreren Spannungsebenen, die zur Überbrückung verschiedener Entfernungen dienen. Transformatoren verbinden die einzelnen Ebenen miteinander. ${ }^{79}$ Zum einen gibt es die Hochspannungsebene $(110 \mathrm{kV}-380 \mathrm{kV})$, die zur Überbruckung von Langstrecken dient. Diese Ebene wird schon lange als Medium für Kommunikationsdienste genutzt, allerdings nur intern zwischen den EVUs. ${ }^{80}$ Zum anderen gibt es die Mittel- und Niederspannungsebene. Die Mittelspannungsebene $(10 \mathrm{kV}-30 \mathrm{kV})$ fuhrt Strom in die Stadt-

76 Hierfur ist eine Errichtung von Nebenstellanlagen neben den Kopfstationen notwendig, die mit dem Kabelnetz verbunden sein müssen. Außerdem müssen für den Rückkanal weitere Verstärker eingesetzt und vorhandene Verstärker umgerüstet werden. Die Anbindung des Teilnehmeranschlusses (Telefon, Fax, Fernsehen) erfolgt uber eine ,Customer Interface Unit'. Vgl. dazu auch Merkt (1998), S. 41 f.

77 Vgl. VPRT (1997), S. 31.

78 Vgl. Distelkamp (1999), S. 47 f. Das ist ein deutschlandspezifisches Problem.

79 Vgl. Zimmermann u.a. (1998), S. 23 f.

80 Die Technik nennt sich Trägerfrequenztechnik auf Hochspannungsleitungen (TFH) und liegt im Frequenzbereich von $15-500 \mathrm{kHz}$. 
gebiete ein. ${ }^{81}$ Die Niederspannungsebene $(0,4 \mathrm{kV})$ verbindet die Ortsnetzstationen mit den einzelnen Hausanschlüssen. Diese Ebene ist für Kommunikationsanbieter insofern von besonderem Interesse, als sie einen direkten Kundenzugang schafft bzw. die letzte Meile überwunden wird.

Das Ziel von PLC ist die unmittelbare Nutzbarmachung von elektrischen Niederspannungsnetzen und Mittelspannungsnetzen für Telekommunikationszwecke. Stromleitungen werden neben ihrer festen Stromfrequenz mit weiteren Frequenzen belegt, über die Telekommunikationssignale gesendet werden.

PLC bringt vom ökonomischen Standpunkt folgende Vorteile mit sich: Es kann bereits bestehende Netzinfrastruktur als Übertragungsmedium genutzt werden, d.h. es fallen keine hohen Investitionskosten mehr an. Auf der anderen Seite ist der Aufbau der Infrastruktur an den Bedürfnissen für die Durchleitung von Strom optimiert und nicht von hochfrequenten Signalen. Dies bringt zahlreiche Störsignale, Reflexionstellen, Sprünge des Wellenwiderstandes, Tiefpasscharakteristik (d.h. die Signaldämpfung ist bei tiefen Frequenzen gering und nimmt bei steigenden Frequenzen $\mathrm{zu}$ ) mit sich. ${ }^{82}$ Weiterhin sind Energieverteilnetze elektromagnetische offene Gebilde. Daraus folgt, daß es bei Hin- und Rücktransport von Signalen über der Erde oder wie innerhalb eines Hauses in einer Schleife zu Störungen durch Aufbau eines elektromagnetischen Feldes kommen kann. Diese Störungen können durch Erdverkabelung minimiert werden (Erde isoliert). Dann hat ein Stromkabel keine schlechteren Eigenschaften als ein Koaxialkabel.

Potentielle Einsatzgebiete von PLC für Datenübertragung befinden sich auf der Mittel- und Niedrigspannungsebene. ${ }^{83}$ Auf der Mittelspanungsebene ist PLC bereits bis zur Marktreife entwickelt. Es wird eine maximale Übertragungsrate von ca. $2 \mathrm{Mbit} / \mathrm{s}$ unter einer Reichweite von 500 Metern erzielt. D.h. es handelt sich eher um Nischenprodukte. Ein flächendeckender Aufbau eines Telekommunikationsnetzes auf Basis von Mittelspannungsleitungen ist wirtschaftlich nicht als sinnvoll anzusehen. Potentielle Einsatzgebiete können die Betreuung selektiver Kunden sein, wie gewerbliche Stromkunden (Bürohäuser, Kaufhäuser, mittelständische Betriebe). Es kann als Lückenschluß in Telekommunikationsnetzen für neue City Carrier dienen, die in kurzer Zeit ein leistungsfähiges Netz aufbauen wollen.

Das künftige Hauptanwendungsfeld von PLC in bezug auf die Weiterleitung von Telekommunikationsdiensten wird auf der Niederspannungsebene sein. Die Einspeisung von Kommunikationssignalen findet in den Trafostationen statt. Die Trafostationen sind wiederum mit LWL an eine PLC Hauptstation angeschlossen.

\footnotetext{
81 Bislang wurde diese Ebene für unidirektionale Übertragung mit Hilfe der TonfrequenzRundsteuertechnik (TRT) genutzt.

82 Vgl. dazu Aufzählung an Störanfälligkeiten Zimmermann u.a. (1998), S. 26.

83 Vgl. Stamm (2000), S. 7 ff.
} 
Dort haben sie eine Verbindung mit dem Internet und dem Telefonnetz. Ein Signal wird auf dem LWL des Niederspannungsnetzes uber eine Trafostation an den Hausanschluß transportiert. Dort koppelt sich das Signal vor dem Stromzähler aus und wird auf Telekommunikationsleitungen oder ein PLC System furs Haus weitergeleitet. Bisher wurde eine Kapazität von 1,5-2 Mbit/s und $8 \mathrm{Mbit} / \mathrm{s}$ realisiert. Die theoretische Kapazitätsgrenze der verschlüsselten Paketvermittlung liegt bei mehreren $100 \mathrm{Mbit} / \mathrm{s}$, d.h. daß es nicht nur für die reine Sprachübermittlung eingesetzt werden kann, sondern aufgrund seiner hohen kapazitären Leistungen sich unbedingt furr Internet und Breitbanddienste eignet. Dabei existiert folgendes Problem: Das momentan verfügbare Frequenzspektrum für PLC liegt zwischen 9 und $148,5 \mathrm{kHz}$. Dies ermöglicht nur eine Übertragungsrate von einigen $10 \mathrm{kbit} / \mathrm{s}$, das für reine Sprachübertragung ( $20 \mathrm{kbit} / \mathrm{s}$ ) ausreicht, nicht mehr aber für breitbandige Telekommunikationsdienste (mindestens $64 \mathrm{kbit} / \mathrm{s}$ ). Damit PLC bei Markteintritt in den Telekommunikationsmarkt zukunftsorientiert wettbewerbsfähig ist, ist ein wesentlich breiteres Frequenzband von $148,5 \mathrm{kHz}$ bis zu $20 \mathrm{MHz}$ erforderlich, um hochbitratige Daten übertragen zu können. Die Freigabe dieser Frequenzen muß erst beantragt werden. ${ }^{84}$

Hochratige PLC-Systeme für den Local Loop sind derzeit noch nicht marktreif. ${ }^{85}$ Erklärtes Ziel war im Laufe des Jahres 2001 PLC-Systeme für den Niederspannungsbereich in Serienproduktion herzustellen und anzubieten. ${ }^{86}$

\subsubsection{INTERNET TELEFONIE}

Telefonie über das Internet (IT) ${ }^{87}$ greift auf eine andere Netzstruktur zurück als die historischen Telekommunikationsnetze, nämlich auf die der Rechnernetze

84 Vgl. Zimmermann (1998), S. 23 und Wickert-Nick, (1999), S. 45. Der Frequenznutzungsplan ist bei der Regulierungsbehörde einsehbar. Im Zuge der technischen Entwicklungen ist im Mai 2001 die Frequenzbereichszuweisungsplanverwendung in Kraft getreten. Stellungnahmen sind in Amtsblatt 16/2000 Mitteilung 507/00; 24/2000 Mitt. 738/00; 5/2001 Mittel. 122/01 nachlesbar.

85 Vgl. RegTP (2001a), S. 20.

86 Die erste Pilotstrecke der Firma Alcatel im Zusammenhang mit Stadtnetzbetreiber R-KOM in Regensburg (vgl. Borchers (1999), S. 41) war offenbar sehr erfolgreich in bezug auf Qualităt, Verfügbarkeit und Ausfallsicherheit. Ein weiterer Feldversuch fand in Rosenheim im Hinblick auf die Tauglichkeit des Access-Systems HYTAS (Hybrides Teilnehmeranschlußsystem) statt. Dort wurde für die lokale Telekommunikationsmesse RO-BIT ein Fast Internet Access über die Energieversorgungskabel der Stadthalle erprobt. Weitere Pilotstrecken gibt es von Tesion in Herrenberg, Pfinztal-Berghausen und Sinzheim. Es wurden 150 Kunden an PLC zur Nutzung von Sprachtelefonie und Breitbanddienste wie InternetAnschlußangeschlossen. RWE $A G$ ermöglicht seit dem Frühjahr 2000 PLC- Anschlüsse für 200 Teilnehmer in Essen.

87 Internet Telefonie wird auch als Voice over Internet Protocol (VoIP) bezeichnet, wobei dieser Begriff auf die Transporttechnologie abstellt. 
bzw. des Internet. Das Internet bezeichnet einen dezentralen Zusammenschluß von Netzen und angeschlossenen Computern. Es vergrößert sich durch den Anschluß zusätzlicher Rechner und Netze ständig. Das Internet wird in lokale $\left(\mathrm{LAN}^{88}\right)$, regionale $\left(\mathrm{MAN}^{89}\right)$ und Langstreckennetzbereiche $\left(\mathrm{WAN}^{90}\right.$ und $\mathrm{GAN}^{91}$ ) unterteilt. Die Netzstruktur ist folglich hierarchisch gegliedert. Die Daten- und Sprachübermittlung via Internet funktioniert im Teilnehmeranschlußbereich über Kupferkabel sowie über LWL.

Der Ausbau der Rechnernetze und der Datenkommunikation hat parallel zum Telefonnetz stattgefunden. Das Übertragungsverfahren funktioniert nach dem Prinzip der verbindungslosen Kommunikation ${ }^{92}$. D.h. bei der Kommunikation zwischen Rechnern werden Daten übertragen, die nur zeitweise eine Übertragungskapazität benötigen. Deswegen wurde für die Rechnernetze der asynchrone, paketorientierte Transfermodus (PTM) entwickelt. Die Vermittlung funktioniert in Form von Routing, d.h. einer gezielten Weitergabe der Nachrichtenpakete bis zum nächsten Knoten, bis es das Ziel erreicht hat. Dabei wird Sprache wie bei Datenmengen in ,Internet Protocol' (IP) -Paketen verschickt.

Die weite Verbreitung der lokalen Rechnernetze (LAN) hat dazu beigetragen, daß das Netz stark dezentrale Strukturen aufweist mit kleinen aber sehr hochfunktionalen Vermittlungsknoten.

88 Das LAN - Local Area Network - ist ein räumlich eng begrenztes Netz. Es überträgt digitale Signale mit hoher Übertragungsgeschwindigkeit. Dabei werden die zu transportierenden Informationen als individuell unabhängige Pakete vermittelt. Die Pakete erhalten neben der Information den Absender und die Adresse. Es gibt keinen gezielten Verbindungsaufbau und abbau bei der Bereitstellung einer Kommunikationsverbindung zwischen Sender und Empfänger (d.h. der Transportdienst erfolgt verbindungslos). Die Pakete suchen sich anhand der Adresse einen eigenen Weg im Netz. Ein typisches LAN ist das Ethernet. Ethernet ist ein eingetragenes Warenzeichen der XEROX Corporation. Es handelt sich hierbei um ein Liniennetz mit verteilter Steuerung nach dem Standard IEEE 802.3 (IEEE - Institute of Electrical and Electronic Engineers).

Das MAN - Metropolitan Area Network - ist ein regionales Hochgeschwindigkeitsnetz. Es verbindet lokale Rechnernetze miteinander mit Hilfe standardisierter Übertragungseinrichtungen. Die Technik dieser Netzes ermöglicht eine Eingliederung der Breitbandnetze. Das Datex- $M$ ist ein Hochgeschwindigkeitsnetz der Telekom und existiert seit 1993. Es überträgt bis zu 140 Mbit pro Sekunde. Die Zugänge zum Datex-M Netz können vom Ethernet, Token Ring oder aus dem synchronen Netz erfolgen.

Das WAN- Wide Area Network - ist ein flächendeckendes Telekommunikationsnetz. Es umfaßt spezialisierte Arten verschiedener Netze wie Fernsprechnetze oder Datennetze. Das WAN überfuhrt bestehende Netze in ein einziges Netz, in dem alle Telekommunikationsdienste abgewickelt werden können, das durch digitale Vermittlungs- und Übertragungssysteme ermöglicht wird.

91 Das GAN - Global Area Network - ist ein interkontinentales Netz und besteht hauptsächlich aus Satellitenstrecken und Überseekabel.

92 Es gibt keinen gezielten Verbindungsaufbau und -abbau bei der Bereitstellung einer Kommunikationsverbindung zwischen Sender und Empfänger. 
Computer zu Computer Internet Telefonie (C-C-IT):

Es bedarf zweier Computer, die mit der entsprechenden Hard- und Software ausgestattet sind. Der Anrufempfanger muß zum Zeitpunkt des Anrufs seinen Rechner angestellt haben, die entsprechende Software geladen und am Internet angeschlossen (online) sein. D.h. der Anrufer wie der Angerufene sind zum entscheidenden Zeitpunkt an einen Internet Service Provider (ISP) uber ihren Teilnehmeranschluß oder eine Mietleitung angeschlossen, so daß der Zugang zum Internet Backbone gewährleistet ist. Der Zugang zum ISP erbringt ein Telekommunikationunternehmen. In der Regel ist das das Unternehmen, das den Teilnehmeranschluß bereitstellt, also in den meisten Fällen der Incumbent. Der ISP erbringt den IP-Transport (routing) im Fernverbindungsnetz. Die C-C-IT eröffnet den Gesprächsteilnehmern die Möglichkeit, neben dem Telefongespräch noch andere IP-Dienste zu nutzen, z. B. Videokonferenzen, Call Center Anwendungen, voice-mail, e-mail und Fax.

Computer zu Telefon Internet Telefonie (C-T-IT):

Der Anrufer initiiert das Gespräch vom Computer aus, d.h. er ist über einen Anschluß an einen ISP und damit an das Backbone angeschlossen. Da das Gespräch an einem Telefon uber das Public Switched Telephone Network (PSTN) terminiert werden soll, muß es vorher umgewandelt werden. Ein Internet Telefonie Service Provider (ITSP) stellt den benötigten Voice Gateway bereit. Ein Gespräch von Telefon zu Computer ist theoretisch möglich, allerdings nicht mit einem gewöhnlichen Telefon. Es muß sich hierbei um ein Telefon handeln, das eine IP- oder E-mail Adresse ansteuern kann.

Telefon zu Telefon Internet Telefonie (T-T-IT):

Hierbei erfolgt sowohl die Initiierung als auch die Terminierung über das PSTN. Beide Gesprächsteilnehmer müssen ein Voice Gateway über einen ITSP erreichen können. Dafür ist kein Internet- Zugang und keine entsprechende Hard- und Software nötig. Die Gesprächspartner müssen die Übertragungsleistung der jeweiligen Telefongesellschaften in Anspruch nehmen, die den Transport zum ITSP gewährleisten. Der ITSP wandelt Sprache in IP-Pakete um. Der Transport erfolgt über das IP im Backbone. Die Telefon zu Telefon Internet Telefonie geht mit herkömmlichen Endgeräten. Dann können aber auch nur die Eigenschaften des herkömmlichen Telefondienstes genutzt werden.

\section{Tabelle 3.4-1: Formen der Internet Telefonie}

Die ersten kommerziellen Vernetzungskonzepte zwischen Rechnern stammen von den Rechnerherstellern. Deswegen gab es zunächst firmenspezifische Protokolle. Um eine Vernetzung unterschiedlicher Hersteller zu ermöglichen, wurden die Protokolle mit Hilfe des ,Open System Interconnection' (OSI) Referenzmodells standardisiert. ${ }^{93}$ Das OSI-Referenzmodell ist ein Kommunikationskonzept für offene Kommunikationssysteme in der Datenkommunikation. Es wurde 1983 von der ,International Standard Organization“ (ISO) ratifiziert und hat sich inzwischen als ein allgemein anerkannter Standard durchgesetzt. Die technischen Ziele des Modells sind, einen einheitlichen Gestaltungsrahmen vorzugeben, in dem Kom-

93

Vgl. HaaB (1997), S. 115. 
munikationsdienste und Protokolle festgelegt, die technischen Ausführungen jedoch noch frei wählbar sind. Auf diese Weise sichert es die Kompatibilität unterschiedlicher Systeme und ermöglicht den Telekommunikationsanwendern eine breite Vielfalt der Nutzung kompatibler Produkte und Systeme unterschiedlicher Hersteller.

In der Tabelle 3.4-1 werden drei verschiedenen Formen der Internet Telefonie ${ }^{94}$ genannt, die sich dahingehend unterscheiden, ob die Sprachübertragung über Computer oder über IT kompatible Telefone stattfindet.

$\mathrm{Zu}$ den rechtlich-regulatorischen Rahmenbedingungen sei darauf hingewiesen, $\mathrm{da} \beta$ es bislang noch umstritten ist, ob es sich bei Internet Telefonie über IP um Sprachübertragung handelt. Solange dies nicht entschieden ist, sehen regulatorische Instanzen wie die EU-Kommission oder die FCC davon ab, die Regulierung von Telekommunikationunternehmen auf die Anbieter von Internet Telefonie zu übertragen. Dies führt durchaus zu regulierungsbedingten Preisvorteilen, da die Betreiber von Internet Telefonie keinen, Accouting Rate Regimes' unterliegen.

\subsubsection{FUNKGESTÜTZTE ANSCHLUBTECHNOLOGIEN}

Neben kabelgebundenen Anschlußtechnologien gibt es ebenso die Möglichkeit, die letzte Meile über Funksysteme zu überwinden, d.h. der Teilnehmeranschluß ist bis zur ersten Vermittlungsstelle funkgestützt. An der Vermittlungsstelle werden die zu übermittelnden Daten in Kabel eingespeist. Der Begriff der funkgestützten Anschlußtechnologien umfaßt alle in das öffentliche Netz vermittelnden Funksysteme, die sich in ihrem Frequenzbereich, Bandbreitenbedarf, Reichweite, Übertragungssicherheit usw. unterscheiden.

\subsubsection{RICHTFUNK ( WIRELESS LOCAL LOOP )}

Bei der WLL- Technologie handelt es sich um Punkt-zu-Mehrpunkt-Richtfunk (PMP-Rifu). Hierbei substitutiert ein drahtloser Telefonanschluß mit Hilfe stationärer Funktelefonsysteme den Draht von der Ortsvermittlung zum Telefonkunden. Teilnehmer verfügen über mit Antennen ausgestattete Sende- und Empfangsgeräte. Die Anschlüsse stehen mit einer Funkbasisstation in Kontakt, die wiederum in der Regel an die OVSt über ein Erdkabel angeschlossen ist. ${ }^{95}$

\footnotetext{
94 Zu einer ausführlichen Darstellung der verschiedenen Internet-Telefonie- Formen vgl. Kuhlenkampff (2000), S. 10 ff. sowie zu Unternehmenstrategien von IT Anbietern vgl. Kuhlenkampff (2000), S. $29 \mathrm{ff}$.

95

Vgl. Merkt (1998), Distelkamp (1999), S. 59.
} 
Die RegTP entschied sich zu einem mehrstufigen Vergabeverfahren fur die in Frage kommenden Funkfrequenzen für die drahtlose Anbindung von Teilnehmeranschlüssen. Sie begann mit einem Antragsverfahren (10.07.1998), über das die Nachfrage nach Frequenzen in den verschiedenen Versorgungsbereichen ermittelt werden sollte (1. Stufe). In den Ballungsgebieten Deutschlands gab es mehr Anträge als verfügbare Frequenzen, so da $\beta$ ein Ausschreibungsverfahren (25.03.1999) durchgefuhrt wurde (2. Stufe). 26 Frequenzblöcke wurden in den Bereichen $2,6 \mathrm{GHz}, 3410$ bis $3,5 \mathrm{GHz}$ und im $26 \mathrm{GHz}$-Bereich ausgeschrieben. ${ }^{96}$ 162 Zuteilungsmöglichkeiten wurden für drahtlose Teilnehmeranschlußleitung für 143 Versorgungsbereiche vergeben. Dabei blieb keine Region ohne Frequenzzuteilung. ${ }^{97} \mathrm{Da}$ für bestimmte Vergabemöglichkeiten keine Bewerbungen vorlagen, wurde im Juni 2000 erneut ein Ausschreibungsverfahren begonnen, und aufgrund einer Optimierung der Frequenzplanung wurden weitere Frequenzzuteilungen ermöglicht (3. Stufe). ${ }^{98}$ Im Dezember 2000 hat die deutsche Regulierungsbehörde über die Zuschläge entschieden. Dabei waren 6 Unternehmen erfolgreich. Bis Mai 2001 sind 800 WLL-PMP- Richtfunkanlagen in Betrieb genommen worden. ${ }^{99}$

Kurzfristig kann es zu Engpässen in bezug auf die Frequenzkapazităt kommen. Die Kapazităt ist in der Regel durch Vorgabe eines Frequenzspektrums zunächst begrenzt. Mittelfristig kann allerdings bei funkbasierten Zellularsystemen durch eine Veränderung der Kodierungs- und Vermittlungsverfahren (Frequenz- und Zeitmultiplexen) und eine Verringerung der Zellgröße die Kapazität erhöht werden, d.h. langfristig wäre die zur Verfuggung stehende Frequenzbandbreite variabel. $^{100}$

\subsubsection{MOBILFUNK}

Eine weitere Möglichkeit eines drahtlosen Teilnehmeranschlusses besteht in der Nutzung von GSM-Lizenzen. Hierbei handelt es sich um die 2.Generation des Mobilfunkstandards. Es ist eine digitale Übertragungstechnik. Sie unterstützt eine

96 Hierbei handelt es sich um sehr hohe Frequenzbereiche, d.h. die Freifelddämpfung ist höher und die Gebăudedurchdringung schlechter. Daraus folgen ein höherer Aufwand bei der Antenneninstallation, geringere Reichweite und dadurch bedingt ist eine erhöhte Zahl an Funkbasisstationen notwendig. Vgl. Graubner (1999), S. 41. Vgl. RegTP (2001b), S. 128 f. Im Mai 2001 entsprach die Summe an Zuteilungen in den betroffenen GHz Bereichen 1671. Vgl. ebenso RegTP Pressemitteilung 25.08.1999.

98 Es gab 503 Bewerbungen von 14 Unternehmen auf die Frequenzzuteilung.

99 Ende September 1999 nahm Viag Interkom als erstes Unternehmen das erste Ortsnetz auf PMP-Rifu-Basis in Landshut in Betrieb. Weitere Testnetze gab es in Dusseldorf und Wiesbaden (STAR ONE).

Vgl. Merkt (1998), S. 90. 
schnelle Übertragung, aber keine hohen Übertragungsdaten. Insgesamt lag Ende 2001 die Penetrationsrate in der deutschen Bevölkerung bei $73 \% .{ }^{101}$

Im August 2000 wurden Lizenzen für die dritte Mobilfunkgeneration - UMTS (Universal Mobile Telecommunications Systems) - versteigert. Neben den Unternehmen, die bereits GSM-Lizenzen haben, haben außerdem noch zwei weitere Unternehmen UMTS- Lizenzen erhalten, nämlich Group $3 G$ und MobilCom. In Europa wird angestrebt, daß UMTS- Dienstebereitstellung im Jahr 2002 möglich ist. UMTS ermöglicht mit Übertragungsraten bis zu 2Mbit/s Breitbandkommunikation über Mobilfunknetze. Sprache wird mit deutlich höherer Qualität übertragbar sein als mit dem GSM-Standard. Es ist multimediafähig, d.h. es unterstützt Internet und Intranet- Zugänge auf Basis des IP, Diensteportabilität und digitale Dienste, weil es volldigital bis zum Kundenanschluß arbeitet.

\subsubsection{MARKTABGRENZUNG ALTERNATIVER ANSCHLUbTECHNOLO- GIEN ZUR HERKÖMMLICHEN FESTNETZTELEFONIE}

Im folgenden sollen die hier vorgestellten alternativen Anschlußtechnologien zur herkömmlichen Festnetztelefonie im Markt für Teilnehmeranschlüsse und Ortsgespräche abgegrenzt werden. Dabei soll gemäß dem Konzept des hypothetischen Monopolisten Nachfrage-, Angebotssubstitutionsbereitschaft sowie potentieller Wettbewerb abgeprüft werden.

Im Rahmen einer horizontalen Abgrenzung bezüglich des sachlich relevanten Marktes soll die Tabelle 3.4-2 zur Hilfe genommen werden. Alle alternativen Technologien können in den Markt für Sprachtelefonie insbesondere im Markt für Teilnehmeranschlüsse und Ortsgespräche eintreten. Weiterhin besteht für alle Anschlußtechnologien bis auf WLL und dem GSM - Mobilfunkstandard technisch die Möglichkeit eines Markteintritts auf den Märkten für sprach-, daten- und videobasierten Mehrwertdiensten.

101 GSM-Standard [890-915 MHz upstream und 935-960 MHz downstream (Vgl. Distelkamp (1999), S. 61)] mit den frequenztechnischen Varianten DCS $1800(1710-1880 \mathrm{MHz})$ und PCS 1900 (1810-1990 MHz), US-Standard D-AMPS (824-894 MHz). Die GSM-Lizenzen werden derzeit von vier Anbietern gehalten DeTeMobil - Deutsche Telekom MobilNet mit 39,9\% Marktanteil 2001, Mannesmann Mobilfunk mit 39,2\% Marktanteil 2001, E-Plus Mobilfunk mit 13,9\% Marktanteil und Viag Interkom als jüngster Anbieter mit 7,2\%. Vgl. RegTP (2001b), S. $191 \mathrm{ff}$. 


\begin{tabular}{|l|c|c|c|c|cr|}
\hline & TV-Kabel & Powerline & IT & WLL & \multicolumn{2}{|c|}{ Mobilfunk } \\
\hline Sprachtelefonie & $\checkmark$ & $\checkmark$ & $\checkmark$ & $\checkmark$ & $\checkmark$ & $\checkmark$ \\
\hline $\begin{array}{l}\text { Sprachbasierte } \\
\text { Mehrwertdienste }\end{array}$ & $\checkmark$ & $\checkmark$ & $\checkmark$ & - & $\checkmark$ & $\checkmark$ \\
\hline $\begin{array}{l}\text { Datenbasierte } \\
\text { Mehrwertdienste }\end{array}$ & $\checkmark$ & $\checkmark$ & $\checkmark$ & - & - & $\checkmark$ \\
\hline $\begin{array}{l}\text { Videobasierte } \\
\text { Mehrwertdienste }\end{array}$ & $\checkmark$ & $\checkmark$ & $\checkmark$ & - & - & $\checkmark$ \\
\hline
\end{tabular}

Tabelle 3.4-2: Horizontale Marktabgrenzung lokaler Anschlußtechnologien zur herkömmlichen Festnetztelefonie

\subsubsection{NACHFRAGESUBSTITUTIONSBEREITSCHAFT}

Als Instrument zur Marktabgrenzung soll wiederum das Konzept des hypothetischen Monopolisten verwendet werden. Um die Substitutionsbereitschaft der Nachfrager für Sprachtelefonie zu alternativen Technologien im Teilnehmeranschlußbereich analysieren zu können, soll von der Vorstellung ausgegangen werden, daß die alternativen Netzbetreiber schon in den Markt eingetreten seien. Es stellt sich die Frage, ob Nachfrager bei einer dauerhaften Preiserhöhung von 5$10 \%$ seitens des Incumbents den Anschlußbetreiber wechseln würden.

In Tabelle 3.4-3 werden die in Kap. 3.3.2 eingefuhrten Kriterien, die die Wechselbereitschaft der Nachfrager auf alternative Anschlußtechnologien beschreiben, analysiert. Sind die Leistungen des Wettbewerbers mit denen des hypothetischen Monopolisten vergleichbar, ist dies in der Tabelle mit , =" gekennzeichnet. Werden die Leistungen höher/schlechter bewertet, werden sie mit einem , +/- ' versehen.

Beim TV-Kabel muß unterschieden werden, ob eine Umrüstung in Form von ,telephony over coax' (t.o.c.) erfolgt oder der overlay approach (o.a.) gewählt wird. Bei beiden muß der Kunde sich ein Modem anschaffen, hat dafür aber keinen zusätzlichen Aufwand bei der Gesprächsinitiierung. Beim t.o.c. wäre die Sprachqualität aufgrund der hohen Ausfallwahrscheinlichkeit zwar eine schlechtere gegenüber dem o.a. und gegenüber dem hypothetischen Monopolisten. Dafür wiederum ist die maximale Datenrate eine deutlich höhere ohne eine Beschränkung hinsichtlich der Übertragungsentfernung. Aus der hohen zur Verfügung stehenden Kapazität ergibt sich die Möglichkeit erweiterter Leistungsmerkmale in bezug auf das zusätzliche Angebot von Breitbanddiensten. Bei einer Preiserhöhung seitens des hypothetischen Monopolisten würde ein Wechsel eines Kunden zum Wettbewerber bei ausschließlicher Nutzung von Sprachtelefonie nicht 
zwangsläufig erfolgen. Bei der Inanspruchnahme von daten- und videobasierten Mehrwertdiensten wären die Kunden eher bereit, zum Wettbewerber trotz höherer Ausfallwahrscheinlichkeit zu wechseln, weil die Datenratenkapazität eine deutlich höhere gegenüber dem hypothetischen Monopolisten ist. Bei dem ,overlay approach' stünde ein identisches Produkt dem des Incumbents gegenüber, da hier ebenso eine Kupferdoppelader für die Übertragung zur Verfügung stünde. Eine Preiserhöhung seitens des hypothetischen Monopolisten würde also unmittelbar einen Wechsel des Kunden bewirken, solange ,switching costs' vernachlässigt werden.

\begin{tabular}{|c|c|c|c|c|c|}
\hline \multirow{2}{*}{\begin{tabular}{|l|} 
Alternative \\
Netzbetreiber \\
/ Kriterien \\
\end{tabular}} & TV-Kabel & \multirow[t]{2}{*}{ Powerline } & \multirow[t]{2}{*}{ IT } & \multirow[t]{2}{*}{ WLL } & Mobilfunk \\
\hline & t.o.c. & & & & GSM UMTS \\
\hline Investitionen & \multirow{2}{*}{$\begin{array}{c}\text { Kauf eines } \\
\text { Modems } \\
\text { erforderlich }{ }^{102}\end{array}$} & \multirow[t]{2}{*}{ keine } & \multirow{2}{*}{$\begin{array}{l}\text { C-C-IT: Software } \\
\text { C-T-IT: IP-Tele- } \\
\text { fon } \\
\text { T-T-IT: keine }\end{array}$} & \multirow{2}{*}{$\begin{array}{l}\text { Kauf einer Emp- } \\
\text { fangsstation er- } \\
\text { forderlich }\end{array}$} & Kauf eines \\
\hline des Kunden & & & & & $\begin{array}{c}\text { GSM UMTS } \\
\text { kompatiblen } \\
\text { Handys } \\
\text { erforderlich }\end{array}$ \\
\hline $\begin{array}{l}\text { Zusätzlicher } \\
\text { Aufwand bei } \\
\text { Gesprächs- } \\
\text { initilerung }\end{array}$ & $=$ & $=$ & + & $=$ & \\
\hline $\begin{array}{l}\text { Sprachquali- } \\
\text { tat }\end{array}$ & - & - & - & - & - \\
\hline $\begin{array}{l}\text { Max. Datenra- } \\
\text { te in Mbit/s }\end{array}$ & \begin{tabular}{|lll}
30 down & 6 \\
$4^{103}$ up & 2
\end{tabular} & hoch & hoch & $\begin{array}{c}\text { kurzfristig nie- } \\
\text { drig begrenzt } \\
\text { langfristig } \\
\text { mittel }\end{array}$ & niedrig \\
\hline $\begin{array}{l}\text { Übertra- } \\
\text { gungsentfer- } \\
\text { nung fur max. } \\
\text { Datenrate } \\
\end{array}$ & k. B. ${ }^{104} 1,8-3 \mathrm{~km}$ & $\begin{array}{l}\text { keine Be- } \\
\text { schränkung }\end{array}$ & $\begin{array}{l}\text { keine Beschrän- } \\
\text { kung }\end{array}$ & $\begin{array}{c}\text { beschränkt, } \\
\text { abhängig vom } \\
\text { Frequenzspek- } \\
\text { trum }\end{array}$ & $\begin{array}{l}\text { keine Beschrän- } \\
\text { kung }\end{array}$ \\
\hline $\begin{array}{l}\text { Erweiterte } \\
\text { Leistungs- } \\
\text { merkmale }\end{array}$ & $+{ }_{\begin{array}{c}\text { Breitband- } \\
\text { dienste }\end{array}}=$ & $\begin{array}{c}+ \\
\text { Breitband- } \\
\text { dienste }\end{array}$ & $\begin{array}{c}+ \\
\text { Breitband- } \\
\text { dienste }\end{array}$ & keine & $\begin{array}{c}++ \\
\text { ständige Erreich- } \\
\text { barkeit }\end{array}$ \\
\hline
\end{tabular}

Tabelle 3.4-3: Kriterien der Nachfragesubstitutionsbereitschaft hinsichtlich lokaler Anschlußtechnologien im Vergleich zur Festnetztelefonie

Bei Powerline muß der Kunde keine zusätzlichen Investitionen leisten und hat keinen zusätzlichen Aufwand bei der Gesprächsinitiierung. Die Sprachqualität bei Powerline auf der Niederspannungsebene ist im Vergleich zur herkömmlichen Festnetztelefonie schlechter. PLC bietet dahingehend einen Qualitätsvorteil, daß

\footnotetext{
102 Vgl. Distelkamp (1999), S. 64.

103 down = downstream; up = upstream

104 k.B. - keine Beschränkung
} 
der Telefonanschluß innerhalb des Hauses uber jede Steckdose gewährleistet ist. Infolge einer Preiserhöhung seitens des Incumbents wird ein Konsument dann wechseln wollen, wenn die schlechtere Sprachqualität die Flexibilität hinsichtlich des hausinternen Anschlusses kompensieren kann. Aufgrund der Möglichkeit, sehr hohe Datenraten bei einer unbegrenzten Reichweite im Vergleich zum hypothetischen Monopolisten ubertragen zu können, hätte Powerline vor allem im Markt für datenbasierte Mehrwertdienste gegenüber dem Incumbent Vorteile.

Bei der Internet Telefonie hängen die zusätzlichen Investitionen eines Kunden von der IT- Form ab, die er wählt. Bei der reinen computerbasierten Internet Telefonie müssen sich die Kunden die entsprechende Software auf ihre PCs laden. Bei Computer zu Telefon IT muß es sich um ein Telefon handeln, das IP entschlüsseln kann. Nur bei der IT von Telefon zu Telefon erfordert es keine zusätzlichen Investitionen seitens des Kunden. Internet Telefonie bedarf eines hohen zusätzlichen Aufwands bei der Gesprächsinitiierung. Bei IT von PC zu PC müssen beide PCs hochgefahren und entsprechende Software geladen sein. Bei IT über Telefon zu Telefon ist eine Zwischenschaltung uber ein Voice Gateway zu einem ITSP erforderlich. ${ }^{105}$ Die Internet Telefonie ist für die reine Sprachübertragung momentan eher ein weniger relevantes Substitut, weil die Sprachqualität zudem noch schlecht ${ }^{106}$ ist. In dem Markt für datenbasierte Mehrwertdienste ergibt sich allerdings ein anderes Bild. Man spricht von dem Angebot integrierter VoIP Dienste. Hier steht die Integrationsfähigkeit vielfältiger Anwendungen im Vordergrund. Sie läßt beliebige Kombinationen von Diensten zu. Im Vergleich zur ISDNTechnologie, d.h. zu Breitbanddiensten herkömmlicher Telefongesellschaften, kann Internet Telefonie als ernstzunehmendes Substitut aufgefaßt werden.

WLL bietet bei zusätzlichen Investitionen des Kunden in Form einer Empfangsstation eine schlechtere Sprachqualităt ohne zusätzlichen Aufwand bei der Gesprächsinitiierung und verfügt über keine besonderen Leistungsmerkmale. Diese Technologie würde folglich für den Konsumenten erst einen Vorzug erreichen, wenn die Preisdifferenz gegenüber dem herkömmlichen Festnetzanschluß hinreichend groß genug ist.

Im Mobilfunk hat der Kunde bis auf den Erwerb eines Standard- kompatiblen Handys, die in der Regel bei Vertrăgen mit lăngeren Laufzeiten seitens der Anbieter subventioniert werden, keinen zusätzlichen Aufwand, auch nicht bei der

105 Der Anrufer muß einen zweistufigen Wählvorgang durchlaufen: Zuerst muß eine Verbindung zum ITSP hergestellt werden. Dort müssen Benutzer-ID und PIN eingegeben werden, bevor die eigentliche Rufnummer gewählt werden kann. Vgl. Kuhlenkampff (2000), S. 57.

voc Die für das Internet charakteristischen Protokolle - das TCP/IP im OSI-Schichtenmodell führen bei Internet-Telefonie zu Laufzeitverzögerungen, Paketverlust und Schwankungen bei der Ankunft von Paketen (Jitter). Die Verwendung von UDP anstelle von TCP für Internet Telefonie verringert das Problem. Vgl. dazu Kuhlenkampff (2000), S. 7 ff. 
Gesprächsinitiierung. Die Gesprächsqualität bei dem herkömmlichen GSM Standard im Mobilfunk ist im Vergleich zur Festnetztelefonie schlechter, die Datenraten sind niedriger und die Gesprächsgebühren höher. Die ständige Erreichbarkeit, die der Kunde über den Mobilfunk erwirbt, wirkt sich dafür qualitätssteigernd aus und schafft eine Akzeptanz seitens des Kunden für höhere Gesprächsgebühren als beim Festnetz. Das Verbindungsaufkommen vom Mobilnetz ins Festnetz ist von 56\% von 1999 auf $46 \%$ im Jahr 2000 gesunken. Der Verkehr innerhalb von Mobilfunknetzen ist dagegen in den beiden Jahren von $39 \%$ auf $48 \%$ gestiegen. Im Tätigkeitsbericht der Regulierungsbehörde ist diese Entwicklung als Substitutionseffekt des Festnetzes interpretiert worden ${ }^{107}$. Das hieße, daß es für eine steigende Anzahl an Konsumenten, die sowohl Kunden vom Festnetz als auch vom Mobilfunk sind, attraktiv ist, Festnetzgespräche vom Mobilnetz aus zu substituieren. Dies gilt allerdings nicht für den Markt für datenbasierte Mehrwertdienste. Mit dem Markteintritt des UMTS -Standards wird die Sprachqualität zunehmen und außerdem die maximale Bitratenübertragung ansteigen. Mobilfunk wird dann auch als Anbieter in den Märkten für Breitbanddienste auftreten können.

\subsubsection{ANGEBOTSSUBSTITUTIONSMÖGLICHKEITEN}

Angebotssubstitutionsmöglichkeiten ergeben sich aufgrund kurzfristiger Reaktionen von Anbietern in angrenzenden Märkten auf eine dauerhafte Preiserhöhung des Incumbents, ohne daß bedeutende Anpassungskosten anfallen.

TV-Kabel, Powerline sowie WLL werden kurzfristig noch nicht auf eine Preissenkung des hypothetischen Monopolisten reagieren können, da die Umrüstung bestehender Infrastruktur beim TV-Kabel und bei den Strombetreibern nicht kurzfristig erfolgen kann und zum zweiten die Anpassungskosten nicht unwesentlich sind. Mittelfristig ist eine Reaktion im Bereich der Sprachtelefonie sowie in dem Markt für Breitbanddienste denkbar, wenn die notwendigen netzwerktechnologische Strukturen gegeben sind.

Im Bereich der Internet Telefonie haben die hohen Preise für internationale Telefonie ITSP schon veranlaßt, in den Markt einzusteigen. Sie profitieren zum einen von regulierungsbedingten Preisvorteilen durch eine Umgehung des ,Accounting Rate Regimes', die sonst bei grenzüberschreitendem Telefonverkehr anfallen. ${ }^{108}$ Zum anderen scheint Internet Telefonie im Backbone kostengünstiger auszufallen. Ein Kostenvergleich von Internet Telefonie und PSTN ist diesbezüglich recht auf-

\footnotetext{
107 Vgl. RegTP (2001b), S. $191 \mathrm{ff}$.

108 Vgl. Kuhlenkampff $(2000$, S. 19.
} 
schlußreich. Eine Studie von Rinde (1997), die auf der VON-Konferenz $1997^{109}$ vorgestellt wurde, hat die angefallenen Kosten pro Bit als Vergleichsmaßstab genommen. Bei einem Vergleich der Übertragungskosten im Backbone zwischen PSTN und IP Infrastruktur verursacht IP bei Langstreckenverbindungen uber mehrere tausend Kilometer (coast to coast) geringere Kosten als herkömmliche Sprachübertragung über das PSTN. Bei Übertragungen über ca. $500 \mathrm{~km}$ ist das PSTN günstiger als die Übertragung mittels IP. Die Ergebnisse beruhen auf Investitionskosten von 1997. Es ist anzunehmen, daß die Kosten von VoIP im Verhältnis zum PSTN gesunken sind, da auf dem Markt furr Router und Gateways erhebliche Preissenkungen zu beobachten waren. ${ }^{110} \mathrm{Da}$ im Teilnehmeranschlußbereich Betreiber von Internet Telefonie entbündelte Teilnehmeranschlußleitungen vom Incumbent anmieten und kein eigenes Teilnehmeranschlußnetz aufbauen, sind kurzfristige Reaktionen auf Preiserhöhungen des hypothetischen Monopolisten möglich. ${ }^{11}$ Daraus läßt sich schließen, daß die Internet Telefonie sich als ernstzunehmendes Substitut sowohl im Weitverkehrsbereich als auch im Teilnehmeranschlußbereich entwickeln kann.

Die Substitutionsbereitschaft seitens der Mobilfunkanbieter ist durchaus gegeben. Mobilfunkbetreiber sind in einem angrenzenden Markt zum hypothetischen Monopolisten tätig. VIAG Interkom ist der erste Mobilfunkbetreiber, der mit seinem Produkt „Genion“ einen Mobilfunkanschluß als Substitut zu einem Festnetzanschluß anbietet. In einem Umkreis von 500 Metern, der sogenannten Home Zone, zahlt der Teilnehmer Festnetztarife fur den Mobilfunkanschluß. Allerdings ist über Genion keine Voreinstellung eines alternativen Netzbetreibers (Preselection) möglich und ist deswegen nur begrenzt als Substitut zu einem herkömmlichen Festnetzanschluß zu betrachten. Generell ist Mobilfunk beschränkt zur herkömmlichen Festnetztelefonie substituierbar. Zwar gewinnt der Mobilfunk im Rahmen der dritten Mobilfunkgeneration UMTS mit der Möglichkeit zur Breitbandkommunikation an Qualităt und ist unter diesem Gesichtspunkt vergleichbar mit dem, was ein Festnetzanschluß bietet. Aber Mobilfunk muß eine flächendeckende Funkversorgung gewährleisten. Die Möglichkeit für den Konsumenten, überall erreichbar zu sein, macht den Mobilfunk erstens zu einem anderen Produkt als einen

109

110

Dies gilt nicht für den Teilnehmeranschlußbereich. Eine Studie von Probe Research von 1998 hat die Kosten für Anschlußports miteinander verglichen. Das Ergebnis der Studie lautete, $\mathrm{da}$ der Preis pro Anschlußport für das PSTN wesentlich günstiger ist als fur Internet Telefonie. Vgl. Probe Research (1998). Die Kosten sind auf die Überbruckung in das PSTN zurickzufuhren. Erst bei einem vollständig auf IP umgerüsteten Netz sind die Kosten für die Anschlußports nicht von Bedeutung. Vgl. Kuhlenkampff (2000), S. 17.

III Auf der anderen Seite sind die IT-Anbieter vom Incumbent abhängig, weil sie einen Zugang auf den vorgelagerten Markt benötigen. 
Festnetzteilnehmeranschluß im Haus und läßt zweitens die Kostenstrukturen zu unterschiedlich ausfallen.

\subsubsection{POTENTIELleR WettBeWERB}

Um beurteilen zu können, ob es sich bei den hier vorgestellten alternativen Technologien um potentiellen Wettbewerb $\mathrm{zu}$ dem hypothetischen Monopolisten handelt, werden zentrale Punkte, die bei einem Markteintritt zu beachten sind, aufgezählt, an denen sie in Tabelle 3.4.-4 miteinander verglichen werden können.

\begin{tabular}{|c|c|c|c|c|c|}
\hline $\begin{array}{l}\text { Alternative Netz- } \\
\text { betreiber / } \\
\text { Zentrale Elemen- } \\
\text { te bei Marktein- } \\
\text { tritt }\end{array}$ & TV-Kabel & $\begin{array}{c}\text { Powerline } \\
\text { - PLC }\end{array}$ & $\begin{array}{l}\text { Internet } \\
\text { Telefonie }\end{array}$ & WLL & $\begin{array}{l}\text { Mobilfunk } \\
\text { UMTS }\end{array}$ \\
\hline $\begin{array}{l}\text { Benötigte Zeit } \\
\text { zum Netzaufbau } \\
\end{array}$ & $\begin{array}{c}\text { Netz existiert } \\
\text { schon }\end{array}$ & $\begin{array}{c}\text { Netz existiert } \\
\text { schon }\end{array}$ & $\begin{array}{c}\begin{array}{c}\text { Netze existiert } \\
\text { schon }\end{array} \\
\end{array}$ & $\begin{array}{c}\text { schneller } \\
\text { Netzaufbau }\end{array}$ & im Aufbau \\
\hline $\begin{array}{l}\text { Höhe der irrever- } \\
\text { siblen Investiti- } \\
\text { onskosten }^{1 / 2}\end{array}$ & mittel - hoch & mittel - hoch & gering & gering & hoch $^{113}$ \\
\hline $\begin{array}{l}\text { Teilnehmerabh. } \\
\text { Kosten } \\
\end{array}$ & niedrig & niedrig & niedrig & hoch & hoch \\
\hline Größenvorteile & $\begin{array}{l}\text { mittel - } \\
\text { hoch }^{1 / 4}\end{array}$ & vorhanden & gering-konstant & $\begin{array}{l}\text { gering- } \\
\text { konstant }\end{array}$ & $\underset{\text { konstant }}{\text { gering- }}$ \\
\hline Verbundvorteile & vorhanden & vorhanden & vorhanden & keine & keine \\
\hline
\end{tabular}

Tabelle 3.4-4: Zentrale Elemente für Markteintritt potentieller Wettbewerber

Ein zentrales Element für einen absehbaren Markteintritt eines potentiellen Wettbewerbers ist die benötigte Zeit für einen Netzaufbau. Ein langwieriger Netzaufbau reduziert die Wahrscheinlichkeit eines Markteintritts. Als weitere Kernpunkte dominieren Kostencharakteristika lokaler Anschlußtechnologien ${ }^{116}$ hinsichtlich

112 Irreversible Investitionskosten umfassen sowohl anfallende Kosten bei der Kabelverlegung bzw. Antennenaufbau (Tiefbauarbeiten, Lohnkosten,...) als auch Umrüstungskosten (Kosten in F\&E und des gesamten Material- und Arbeitseinsatzes, um Infrastruktur kommunikationstauglich zu machen).

113 Allein aufgrund der hohen Ausgaben für die Lizenzen, die im Rahmen eines spektakulären Auktionsverfahrens 2000 vergeben wurden.

114 Cave (1995) sagt mittel, Levin u.a. (1998), S. 6, sagen hoch.

IIS Vgl. dazu empirische Studie über den amerikanischen Mobilfunkmarkt von McKenzie/Small (1997).

116 Erste Kostenvergleiche zu finden bei Cave (1995), S. 4. 
eines Netzaufbaus bzw. einer Netzumrüstung im Vergleich zum herkömmlichen Festnetzanschluß. Dabei geht es bei einem Vergleich der Kostenstrukturen nicht um die Angabe exakter absoluter Zahlen, sondern um die Einordnung der Wettbewerbsfähigkeit der verschiedenen Technologievarianten. Die Kostenstruktur und -höhe ermöglichen Vorhersagen über die Marktchancen der verschiedenen Anschlußtechnologien. So ist der Anfall irreversibler Investitionskosten im Hinblick auf einen Markteintritt von außerordentlicher Bedeutung, da mit ihrer Höhe das unternehmerische Risiko steigt und die Wahrscheinlichkeit eines tatsächlichen Markteintritts sinkt und dementsprechend auch der Wettbewerbsimpuls eines potentiellen Wettbewerbers. Je niedriger der teilnehmerabhängige Teil an Infrastrukturinvestitionen einer Technologievariante ist, um so sensibler reagieren die Kosten auf die Marktdurchdringung (Nachfragepenetration). Ein sehr großer Kundenstamm ist dementsprechend notwendig, um Kostendegressionsvorteile zu erzielen. Aus der Notwendigkeit, eine hohe Marktdurchdringung erzielen zu müssen, ergibt sich gegebenenfalls eine Markteintritts- und austrittsbarriere. ${ }^{117}$

Mit Größenvorteilen, hier im Sinne von horizontalen ,Economies of Scale', sind die Realisierung von Stückkostenvorteilen bei zunehmender Produktionsmenge gemeint. Die langfristigen Durchschnittskosten sinken mit jeder weiteren Produktionseinheit, bis die mindestoptimale Betriebsgröße (MOS) erreicht ist.

Verbundvorteile ergeben sich bei Mehrproduktproduktionen. Eine gemeinsame Produktion ist zu geringeren Stückkosten möglich als eine getrennte Produktion.

Im folgenden werden die ausgewählten substitutiven Technologien hinsichtlich der zentralen Elemente für einen Markteintritt gemäß der Tabelle 3.4-4 überpruft.

Kabel-TV- Betreiber haben bei Markteintritt in Telekommunikationsmärkte den Vorteil, daß schon eine Netzstruktur besteht, die allerdings um- oder aufgeruistet werden muß. Da beim t.o.c. die bisherige Netzstruktur genutzt werden kann, halten sich die irreversiblen Investitionskosten verhältnismäßig gering. Beim o.a. dagegen werden für Telekommunikationszwecke neue Kupferkabel verlegt, allerdings in das schon bestehende Röhrensystem, so daß die Investitionskosten geringer als bei einer kompletten Neuverlegung ausfallen. Da die ,sunk costs' in bezug auf die Umrüstung bestehender Netzstrukturen für Betreiber von TV-Kabel recht hoch sind, schwächt es ihren Wettbewerbsdruck als potentielle Wettbewerber auf den hypothetischen Monopolisten. Die teilnehmerabhängigen Investitionen sind bei der Umrüstung von TV-Kabel im Verhältnis zu funkgestützten Anschlußtechnologien niedrig, und es existieren Größenvorteile. Eine Umrüstung wird folglich zunächst nur in dicht besiedelten Regionen vorgenommen werden, um Kostendegressionsvorteile erzielen zu können. TV-Kabelbetreiber können generell bei einem Einstieg in den Telekommunikationsmarkt neben der Rundfunkübertragung

117 Vgl. Distelkamp (1999), S. 66 f. 
zusätzlich interaktive Breitbandienste anbieten. Auf diese Weise wird ihnen die Möglichkeit eröffnet, Verbundvorteile auszuschöpfen:

a) im Bereich der irreversiblen Investitionen: Das schon angelegte Röhrensystem kann bei beiden Möglichkeiten der Aufrüstung doppelt genutzt werden, indem zusätzliche Kabel für Telekommunikationszwecke implementiert werden. ${ }^{118}$

b) im Bereich der reversiblen Kosten durch eine gemeinsame Nutzung von Kabel, Kopfstations-Einrichtungen, Rechnungserstellung, Netzwartung und Kundenbetreuung.

Je höher die Verbundvorteile sind, desto eher verfügen TV-Breitbandkabelbetreiber gegenüber dem Incumbent über einen absoluten Kostenvorteil. Es gibt inzwischen schon Projekte, in denen Kabelnetzbetreiber Internetzugang und Sprachtelefonie anbieten. ${ }^{119}$ Es gibt außerdem eine Reihe von privaten Kabelnetzbetreibern, die Internetzugänge und Sprachtelefondienste über ihre Kabelfernsehnetze anbieten. ${ }^{120}$ Wenn es also zu Markteintritt von TV- Kabelbetreibern in Telekommunikationsmärkten kommt, dann mit dem Ziel, schnell eine hohe Marktpenetration zu erreichen. Da sowohl eine Netzstruktur als auch ein Kundenstamm schon existieren, wäre eine Marktdurchdringung auch zügig erreichbar. Als Voraussetzung für funktionsfähigen Wettbewerb muß allerdings gewährleistet sein, daß eine vollständige Trennung zwischen der Deutschen Telekom und den TVKabelnetzen vollzogen wird und die DTAG keinen Einfluß auf die Verwendung der Kabelfernsehnetze mehr ausübt. Denn die DTAG wird kein Interesse haben, TV-Kabel umzurüsten, um sich selbst Wettbewerb zu machen.

Betreiber von Powerline haben in bezug auf den Netzaufbau den Vorteil, daß deren Netzstruktur wie beim TV-Kabel schon besteht, aber gegebenenfalls um- oder aufgerüstet werden muß, um sie für Telekommunikationsdienste nutzen zu können. Ist die Netzinfrastruktur umgerüstet, kann der Anschluß von Kunden schnell erfolgen. Die Ankoppeleinrichtung und Endgeräte sind bei geringem Personaleinsatz in wenigen Stunden installiert. Die durchzuführenden Umrüstungskosten verursachen im Vergleich zu funkgestützten Anschlußtechnologien hohe irreversible Investitionskosten. Im Vergleich zu einer vollständigen Neuverkabelung sind die Investitionskosten insbesondere im Teilnehmeranschlußbereich wiederum als niedriger einzustufen. Die teilnehmerabhängigen Kosten sind bei PLC ebenso wie beim TV-Kabel niedrig. Zudem erfordert das Bestehen von Größenvorteilen bei

II8 Vgl. Cramer (1994), S. 284; Merkt (1998), S. 87.

119 KDG hat im Rahmen des Kabelprojektes Berlin '99 gemeinsam mit Wohnungsbaugesellschaften und privaten Kabelnetzbetreibern begonnen, die Kabel-TV-Netze zur Versorgung von 600.000 Haushalten in Berlin auszubauen und um Rückkanäle zu ergänzen. Vgl. www.kabel-berlin.de.

120 Zum Beispiel tut dies die TeleColumbus. Gruppe - eine 100\%ige Tochter der Deutschen Bank und mit 2 Mio. Kunden der zweitgrößte private Kabelnetzbetreiber. Weitere sind Concepta Kommunikationstechnik („telefon plus") und Telekabel Service Süd (,fritz-call“). 
Markteintritt eine hohe Marktdurchdringung, um sie abschöpfen zu können. Die EVUs haben ein großes Interesse, sich ein zweites Standbein zu schaffen. Neben der Existenz eines deutschlandweiten Kundenstamms im Teilnehmeranschlußbereich ist eine weitere Verwendungsmöglichkeit ihrer Netzstruktur wegen des Aussschöpfens von Verbundvorteilen attraktiv. Neben der Nutzung gemeinsamer Einrichtungen können außerdem beide Produkte miteinander kombiniert angeboten werden ${ }^{121}$. PLC ist bei einem höherem Frequenzspektrum als momentan zugelassen für hochbitratigen Verkehr geeignet wie Fast Internet, LANKoppelungen, ,High-Speed' Übertragung von Daten und Sprache. ${ }^{122}$

Anbieter von Internet Telefonie haben kaum irreversible Investititonskosten bei Markteintritt in Kauf zu nehmen, weil sie die bestehende Infrastruktur im Teilnehmeranschlußbereich vom Incumbent anmieten können. Beim Übermittlungsprinzip fallen ebenso keine hohen Investitionskosten an, da es aus der Datenvermittlung stammt - das IP. Die teilnehmerabhängigen Kosten sind wie bei allen netzbasierenden Anschlußtechnologien niedrig. Dementsprechend sind die Größenvorteile konstant. Ob Verbundvorteile bestehen, hängt von dem IT Anbieter ab. Handelt es sich bei dem IT Anbieter um Software- und Equipmenthersteller, ergeben sich Kostenvorteile aufgrund vertikaler Integration. Handelt es sich um ISPs oder um Telefongesellschaften, dann ergeben sich Verbundvorteile in bezug auf Kundenbetreuung und Rechnungserstellung.

Richtfunk auf Basis der WLL-Technologie zeichnet sich durch seine schnelle Verfügbarkeit aus ${ }^{123}$, d.h. nach Zuteilung der Funklizenz kann sehr schnell ein Zugangsnetz aufgebaut werden. Die Installation einer Funkbasisstation kann innerhalb von einer Woche aufgebaut sein. Ohne viel Arbeitsaufwand (d.h. geringe Lohnkosten) können 4-10 Teilnehmerabschlußeinheiten pro Tag installiert werden. Außerdem ist seine Verfügbarkeit auch in geographisch, geologisch und morphologisch schwer zu erschließenden Regionen leichter im Verhältnis zum Kabel. Da bei der Installation von Richtfunk keine Verkabelungs- und Wegerechtkosten anfallen, sind die Infrastrukturinvestitionen verhältnismäßig gering und machen den Einsatz von WLL attraktiv. Die Kosten der funkbasierten Netze variieren mit der Verkehrsdichte. Die Investitionshöhe steigt linear mit dem Teilnehmerzuwachs, d.h. das Risiko eines Markteintritts ist verhältnismäßig gering, da zum einen keine hohen Kosten für Anfangsinvestitionen getătigt werden müssen, zum anderen die Installationskosten eines Funkbereichs nicht irreversibel sind. Wechselt ein Kunde, gehen maximal die Installationskosten der Kundenab-

\footnotetext{
121 Ein Beispiel hierfür ist die telefonische Fernsteuerung strombetriebener Produkte.

122 Der Einsatz von PLC brächte neben der Verwendung bestehender Infrastruktur weitere Kostenvorteile mit sich. Unabhängig von der zu überbrückenden Entfernung ist der Materialeinsatz konstant. Vgl. Borchers (1999), S. 40.

Vgl. Graubner (1999), S. 38.
} 
schlußeinheit verloren. Dafür fallen aber hohe teilnehmerspezifische Kosten an. Der Einsatz der WLL - Technologie ist insbesondere bei einer geringen Teilnehmerdichte kosteneffizient bzw. dort, wo große Entfernungen überbrückt werden müssen. In diesem Punkt unterscheidet sich WLL ganz entscheidend von den alternativen kabelgebundenen Technologien. Während sich kabelbasierte Technologien zunächst vorrangig in Ballungsgebieten ausbreiten, damit aufgrund der relativ hohen Investitionskosten die Nachfragepenetration möglichst hoch ist und Skalenerträge ausgeschöpft werden können, hat WLL aufgrund seiner relativ geringen Investitionskosten und konstanten Größenvorteile einen absoluten Kostenvorteil bei Teilnehmeranschlüssen in der Peripherie.

Mobilfunkbetreiber des UMTS-Standards müssen vor Markteintritt zunächst ihr Netz aufbauen. Dabei ist die benötigte Zeit zum Netzaufbau bei allen Betreibern höher als ursprünglich geplant war. Die irreversiblen Investitionskosten sind als hoch einzustufen. Dabei ist ein großer Anteil der ,sunk costs' - nämlich der Erwerb der Lizenzen - schon getätigt. Aber auch die Investitionskosten aus dem Netzaufbau sind im Verhältnis zu WLL hoch, da Mobilfunk ein flächendeckendes Netz für eine erfolgreiche Anwendung aufbauen muß. Die teilnehmerabhängigen Kosten sind aus denselben Gründen wie bei WLL hoch und die Größenvorteile konstant. Verbundvorteile liegen keine vor. Mobilfunk ist, wie schon erwähnt, begrenzt nur als Substitut zu bewerten. Der Markteintritt ist sehr wahrscheinlich, da die Betreiber mit dem Erwerb der Lizenzen den größten Teil der ,sunk costs' schon geleistet haben.

\subsubsection{VERTIKAL SACHLICHE MARKTABGRENZUNG}

Anhand einer vertikal sachlich relevanten Marktabgrenzung lokaler Anschlußtechnologien, wie sie in Tabelle 3.4-5 aufgeführt sind, lassen sich Aussagen über die Wettbewerbsfähigkeit von Netzen machen. Dort werden die Märkte für lokale Originierung und Terminierung und die jeweiligen Teilnehmeranschlüsse der verschiedenen möglichen Netzbetreiber verglichen. ${ }^{124}$

TV-Kabel- ebenso wie Powerline- Betreiber greifen im Teilnehmeranschlußbereich auf ihre eigene Breitbandkabelstruktur zurück. Ihr Vorteil ist, daß beide sowohl über eigene Teilnehmeranschlüsse als auch über eigene Infrastruktur verfügen. Anbieter von Internet Telefonie müssen wie jeder ISP auf die vorhandene Struktur ehemaliger Monopolgesellschaften zurückgreifen, d.h. sie müssen Teil-

$124 \mathrm{Zu}$ einer vertikalen sachlichen Marktabgrenzung von Zusammenschaltungsleistungen vgl. auch Kap. 3.3.3.1. 
nehmeranschlüsse von dem Incumbent anmieten. ${ }^{125}$ WLL Betreiber müssen zunächst eine eigene Infrastruktur im Teilnehmeranschlußbereich aufbauen, bevor sie in den Markt eintreten können. Beim Mobilfunk geht es nicht um einen lokalen Teilnehmeranschluß, sondern daß der Kunde unter Vertrag ist und über ein Handy verfügt. Der GSM-Standard im Mobilfunk verfugt über eine Penetrationsrate von ca. über $70 \%$ in der Bevölkerung. ${ }^{126}$

\begin{tabular}{|l|c|c|c|c|c|}
\hline Markt für/ & TV-Kabel & Powerline & IT & WLL & Mobilfunk \\
\hline $\begin{array}{l}\text { lokale Origi- } \\
\text { nierung \& TN- } \\
\text { Anschlüsse }\end{array}$ & $\begin{array}{l}\text { eigene In- } \\
\text { frastruktur }\end{array}$ & $\begin{array}{l}\text { eigene In- } \\
\text { frastruktur }\end{array}$ & $\begin{array}{l}\text { keine eigene } \\
\text { Infrastruktur }\end{array}$ & $\begin{array}{l}\text { eigene In- } \\
\text { frastruktur }\end{array}$ & $\begin{array}{c}\text { eigene In- } \\
\text { frastruktur }\end{array}$ \\
\hline $\begin{array}{l}\text { lokale Termi- } \\
\text { nierung \& TN- } \\
\text { Anschlüsse }\end{array}$ & $\begin{array}{l}\text { eigene In- } \\
\text { frastruktur }\end{array}$ & $\begin{array}{l}\text { eigene In- } \\
\text { frastruktur }\end{array}$ & $\begin{array}{l}\text { keine eigene } \\
\text { Infrastruktur }\end{array}$ & $\begin{array}{l}\text { eigene In- } \\
\text { frastruktur }\end{array}$ & $\begin{array}{l}\text { eigene In- } \\
\text { frastruktur }\end{array}$ \\
\hline
\end{tabular}

Tabelle 3.4-5: Vertikale sachlich relevante Marktabgrenzung lokaler Anschlußtechnologien

Bei Markteintritt von Internet Telefonie Anbietern liegt aufgrund der Abhängigkeit von der Gewăhrleistung eines Zugangs zum Netz des Incumbents ein ,OneWay-Access ${ }^{6}$ vor. Unter diesen Umständen ist ihre Wettbewerbsfähigkeit von der Ausgestaltung der Zugangsregelungen abhängig. ${ }^{127}$ In den Kapitel 5.1 und 5.2 sollen verschiedene Möglichkeiten der Bestimmung von Zugangsentgelten vorgestellt und hinsichtlich ihrer Eignung für die Einfuhrung wirksamen Wettbewerbs analysiert werden. Käme es zu einem erfolgreichen Markteintritt eines der anderen substitutiven Anbieter, läge ein ,Two-Way-Access' vor, bei dem lokale Netzbetreiber um Anschlüsse konkurrieren. Dabei ergeben sich so viele Märkte, wie es Netzbetreiber im Teilnehmeranschlußbereich gibt, da jeder Teilnehmeranschluß von einem Anbieter monopolisiert wird. ${ }^{128}$ Aus diesem Grund ist die Zusammenschaltung aller Netze aus ökonomischer Sicht aus Gründen des Bestehens von Netzeffekten und Netzexternalităten als sinnvoll anzusehen. ${ }^{129}$ Das TKG sieht allerdings zusätzlich eine Regulierung der Entgelte für die Zusammenschaltung vor, weil die Gefahr preislicher Absprachen in bezug auf die Verhandlung von $\mathrm{Zu}$ sammenschaltungsentgelten zwischen den Betreibern lokaler Anschlußtechnolo-

125 Vgl. Kap. 2.4.2.2 zum Zugang zu Teilnehmeranschlußleitungen.

126 Vgl. RegTP (2001b), S. $191 \mathrm{ff}$.

127 Regelungen des Netzzugangs werden ausführlich in Kap. 2.4.2 dargelegt.

128 Dies gilt unter der Annahme, daß sich ein Kunde nur einen Teilnehmeranschluß von einem Betreiber leistet.

$129 \mathrm{Zu}$ der gesetzlichen Regelung von Zusammenschaltung vgl. Kap. 2.4.3. Zu einer ökonomischen Legitimation der Zusammenschaltung vgl. Kap. 4. 
gien zum Nachteil der Konsumenten besteht. Ob sich aus dieser Gefahr heraus eine Regulierung der Zusammenschaltungsentgelte legitimiert, soll in Kapitel 5.3 ausführlich diskutiert werden. 


\section{ÖKONOMISCHE GRUNDLAGEN VON NETZINFRA- STRUKTUREN}

Bei der Analyse von Telekommunikationsmärkten sollte der Bedeutung der Netzokonomie Rechnung getragen werden. In der Netzökonomie werden besondere Eigenschaften von Gütern, die von Netzinfrastrukturen abhängig sind, analysiert, die ebenso bei der Etablierung von Wettbewerb im Teilnehmeranschlußbereich eine bedeutende Rolle spielen. Kommt es zum Eintritt von alternativen Netzbetreibern in den Markt für Teilnehmeranschlüsse und Ortsgespräche, können Netzeffekte bei Aufbau bzw. Umrustung einer Infrastruktur von Bedeutung sein.

\subsection{EINFÜHRUNG IN DIE NETZÖKONOMIE}

Netze liegen bei Transportsystemen von Gütern, Personen oder Informationen vor. Sie bestehen im herkömmlichen Sinn aus Knoten und Kanten.' Knoten verkörpern die Punkte, die über ein Netz miteinander verbunden werden. Hierbei kann es sich um Personen oder Produkte handeln. Im Telefonnetz betrifft es Endanschlüsse oder Vermittlungsstellen. Kanten sind die Verbindungswege zwischen den Knoten und können materiell im Sinn einer physisch vorhandenen Leitung sein - eines Kommunikationsnetzwerks -, oder sie sind immateriell in Form einer unsichtbaren bzw. virtuellen Verbindung, die sich auf die Komplementarität zweier verschiedener Güter bezieht - dann spricht man von einem Komponentennetzwerk. Knoten und Kanten bestimmen die Struktur eines Netzwerkes.

\subsubsection{KLASSIFIKATION VON NETZEFFEKTGÜTERN}

Bei Netzeffektgutern handelt es sich um Produkte, die in eine Systemtechnologie integriert sind und durch die Merkmale Kanten und Knoten beschrieben werden ${ }^{2}$, d.h. sie stehen innerhalb eines Netzwerkes in einer Wechselbeziehung zueinander. Ein Gut erlangt erst seinen Wert, wenn es in Kombination mit anderen Gütern verwendet wird. Man kann zwei Arten von Netzeffektgütern unterscheiden, die Netzwerke konstituieren:

1. Güter, für dessen Gebrauch Kommunikationsnetzwerke notwendig sind.

Vgl. Jung/Warnecke (1998), S. 92 ff.

$\mathrm{Zu}$ einer grundlegenden Analyse von Netzeffektgütern vgl. Katz/Shapiro (1985) sowie Farrell/Saloner $(1985,1986 \mathrm{~b})$. 
Hier seien als Beispiele Endgeräte von Telekommunikationssystemen - Telefon, Fax, Mailbox, BTX usw.- genannt. Das Netzwerk in der Telekommunikation umfaßt die Gesamtzahl aller Teilnehmeranschlüsse und damit aller Nutzer. Die Zahl der Nutzer definiert dabei die Größe des Netzwerkes. Die Summe der Netzteilnehmeranschlüsse in einem Zeitpunkt wird als installierte Basis bezeichnet.

Solche Netzeffektgüter verfügen folglich neben dem Nutzen aus dem Gut als Gebrauchsgegenstand über einen derivativen Produktnutzen, der sich aus dem interaktiven Einsatz ergibt. Das Gut Telefonanschluß beispielsweise bringt dem Konsumenten nichts, wenn er der einzige Netzteilnehmer ist. D.h. der Nutzen dieser Netzeffektgüter zeichnet sich dadurch aus, daß er sich für den einzelnen Nachfrager aus der Verwendung dieses Gutes durch andere Nutzer bestimmt. Erst wenn möglichst viele über einen Teilnehmeranschluß verfügen, kann ein Netzteilnehmer erstens viele telefonisch erreichen und zweitens von vielen erreicht werden. Es handelt sich hierbei um den Nutzen, der aus der Teilnahme an einem Netzwerk resultiert. Er wird als Netzeffekt bzw. direkter Netzeffekt bezeichnet. Die Höhe dieses Derivativnutzens hängt von der installierten Basis ab, die sich im Zeitablauf ändert, und ist dadurch eine dynamische Größe. Je größer die installierte Basis ist, desto größer ist der Derivativnutzen bzw. der Netzeffekt. ${ }^{3}$ Güter, die solche Netzeffekte auslösen, werden auch ,horizontale Netzprodukte ${ }^{4}$ genannt, da für die Funktionsfähigkeit ein Netzwerk an Produkten auf gleicher Ebene notwendig ist. Horizontale Netzprodukte definieren sich über ihre Nutzensteigerung aufgrund einer Netzausweitung und nicht über ihre Nachfrageausweitung, die aber implizit wegen der Nutzensteigerung erfolgen kann. ${ }^{5}$

2. Güter, die durch ein Komponentennetzwerk miteinander verbunden sind. Der Netzbegriff kann auch weiter ausgelegt werden. Dann können die Abhängigkeiten komplementärer Produkte als Netzwerk bezeichnet werden. Während horizontale Netzprodukte nur Teile eines Systems sind, ist hier jeder Konsument Nutzer eines vollständigen Systems. Das System erreicht seinen Nutzen erst durch die Verbindung kompatibler Komponenten. ${ }^{6}$ Unternehmen bieten entweder das ganze

3 Die Nutzungsintensität der einzelnen Nachfrager spielt hier keine Rolle. Im Rahmen einer anderen Analyse unter einem anderen Gesichtspunkt kann die Nutzungsintensität jedoch von Bedeutung sein. Vgl. Holler (1997), S. 92.

4 Zu einer ausführlicheren Systematik von Netzprodukten vgl. Matutes/Regibeau (1996), S. 185 ff.; Köster (1999), S. 24 ff.

s Rohlfs (1974) zeigt die Konsequenzen von Netzeffekten für eine aggregierte Nachfragefunktion für Telekommunikationsdienste auf. Sie führen dazu, daß multiple Gleichgewichte vorliegen, die unterschiedlich stabil sind. Netzeffekte ziehen potentielle Nutzer an, so daß bei einem stabilen Preisniveau eine geringe Nutzerzahl ein instabiles Gleichgeicht bedeuten würde. Die Nutzerzahl steigt wegen der positiven Netzeffekte sprunghaft an und erreicht bei unverändertem Preisniveau ein stabiles Gleichgewicht.

Vgl. Köster (1999), S. 27. 
System an oder nur einzelne Systemkomponenten. Jene Güter, die zu ihrer Nutzentfaltung ein solches Komponentennetzwerk benötigen, werden auch als ,vertikale Netzprodukte' bezeichnet. ${ }^{7}$ In diesem Zusammenhang wird immer wieder auf das Beispiel von Betriebssystemen und die darauf aufbauenden Anwendungsprogramme verwiesen. In der Regel laufen die Programme nur auf dem einen Betriebssystem und auf keinem anderen. Die Verbreitung der Hardware stellt sozusagen das Netzwerk für die Software dar. Die Hardware ist das primäre Netzwerkgut. Die Software ist das Komplement dazu. Beide Komponenten benötigen sich zu ihrer Nutzentfaltung gegenseitig, d.h. sie stehen in einem komplementären Verhältnis zueinander. PCs sind nicht die einzigen Güter, auf die eine HardwareSoftware-Beziehung zutrifft. Dies gilt beispielsweise auch für Videorecorder und Videokassetten, CD-Player und CDs oder Endgeräte und Teilnehmeranschlüsse usw. Das Gut Telefon ist erst nutzbar, wenn der Konsument auch uber einen Netzanschluß verfügt. Komplementärgüter müssen nicht in einem fixen Verhältnis zueinander konsumiert werden.

Der Derivativnutzen ergibt sich aus dem Nutzernetzwerk komplementärer Produkte. Ein Kunde wird beim Kauf eines Produktes seine Kaufentscheidung von der Größe der installierten Basis des Netzwerkgutes abhängig machen; d.h. ist eine Hardware sehr verbreitet, dann gibt es auch ein zahlreiches und vielfältiges Angebot an Software dazu. Also kauft der Kunde die Hardware, die am meisten verbreitet ist, weil dann auch die Auswahl an Software größer ist. ${ }^{8}$ Das vielfältige Angebot an Software, also des Komplementärproduktes, ist entscheidend für eine Nutzensteigerung des Netzwerkgutes, d.h. der Nutzen aus der Hardware wird durch eine Verbreitung der Komplementärprodukte erhöht. Die Nutzensteigerung erfolgt indirekt. ${ }^{9}$ Man spricht deswegen auch von einem indirekten Netzeffekt.

Dieser Zusammenhang spielt insbesondere für Produkte eine Rolle, die sich noch in einer jungen Marktphase befinden und für die ein starker Wettbewerb zwischen konkurrierenden Systemen besteht. Die Nutzensteigerung der einzelnen Konsumenten liegt in der jeweiligen Marktdurchdringung eines Komplementärproduktes. Je höher die Marktpenetration eines Komplementärproduktes ist,

- desto eher kann es sich auf dem Markt gegenüber konkurrierenden Systemprodukten durchsetzen und als zuküntiger Standard fungieren. Hier steht mehr auf dem Spiel, als nur Marktfuhrer zu werden. Bei nicht kompatiblen Standards uberlebt in der Regel langfristig nur der Standard, der sich auf dem Markt durchsetzt. Für die Konkurrenten bedeutet das, vom Markt verdrängt zu

7 Grundlegende Erkenntnisse bzgl. vertikaler Netzprodukte vgl. Matutes/Regibeau $(1988,1992)$ sowie Economides (1989).

8 Vgl. Katz/Shapiro (1985), S. 424.

9 Hierbei ist wiederum der Kauf des Produktes für den Netzeffekt ausschlaggebend, nicht die Nutzungsintensităt. 
werden, solange die Komplementärprodukte nicht zu verschiedenen Netzwerkprodukten unterschiedlicher Betreiber kompatibel sind. Dies ist nicht nur für die Unternehmen von Nachteil. Auch die Konsumenten, die in diese Technologie investiert haben, haben irreversible Ausgaben getätigt. ${ }^{10}$

- desto höher kann die Servicebetreuung vor Ort sein (z.B. höhere räumliche Abdeckung von Fachgeschäften), die wiederum in die Qualität der Produkte mit einfließt.

Hier sei kritisch angemerkt, daß mit der Definition indirekter Netzeffekte eine Nachfrageinterdependenz umschrieben wird, die in nahezu allen Märkten präsent ist und auf gewöhnliche komplementäre Güter zutreffen kann. Sie verwässert die Abgrenzung zwischen Netzeffektgütern und ,normalen' Gütern. Das birgt die Gefahr, daß herkömmliche Preiszusammenhänge bzw. wohlfahrtsneutrale Interaktionen " plötzlich als Netzeffekte wiederentdeckt werden.

\subsubsection{DEFINITION EINES NETZEFFEKTES}

Es läßt sich festhalten, daß ein direkter wie indirekter Netzeffekt auf Eigenschaften beruht, die ein Produkt erst in Kombination mit einem Netzwerk, welches sich entweder auf Teilnehmer oder auf komplementäre Produkte bezieht, entfaltet. Der Nutzen aus einem Netzeffekt ergibt sich aus dem wie oben schon beschriebenen Derivativnutzen. Bei einem Netzeffekt hängt der Nutzen eines Netzeffektgutes ab von

1. der Zahl der Akteure, die gleichwertige Handlungen durchführen. Ein Beispiel hierfür ist die schon erwähnte Netzgröße eines Kommunikationssytems.

2. der Kompatibilität unterschiedlicher Systemkomponenten. Hierbei wird Kompatibilität unterschieden in ${ }^{12}$

- vertikale Kompatibilität bei komplementären Produkten. Komplementäre Produkte sind vertikal kompatibel, wenn sich z. B. eine Software auf unterschiedliche Betriebssysteme installieren läßt.

- horizontale Kompatibilität bei substitutiven Produkten wie beispielsweise Mobilfunk und Festnetz und bei Netzwerken. Horizontal kompatible Produkte stehen im Wettbewerb zueinander.

- Kompatibilität durch Adapter bzw. Konverter bei Vorliegen von inkompatiblen Systemen (Gateway-Technologie).

10 Vgl. hierzu Kap. 4.1.4.1 und 4.1.4.2.

1 Vgl. Liebowitz/Margolis (1994), S. 139.

12 Vgl. David/Bunn (1988), Holler (1997), S. 93. 


\subsubsection{DEFINITION VON NETZEXTERNALITÄTEN}

Netzeffekte können Netzexternalităten implizieren und sollten nicht mit ihnen verwechselt werden. ${ }^{13}$ Externe Effekte sind aus der Theorie effizienter Allokation bei dezentralen Entscheidungen bekannt. Sie besagt, daß ein Pareto-Optimum nur bei Abwesenheit von externen Effekten vorliegt. D.h. die Existenz von externen Effekten entspricht einem Marktversagen. Aber Mărkte, in denen Netzeffekte existieren, beinhalten nicht zwangsläufig Marktversagen. ${ }^{14}$ Aus diesem Grund sollten Netzeffekte und Netzexternalitäten sprachlich nicht miteinander vermischt werden. Als Netzexternalität sollte der positive externe Effekt bezeichnet werden, den ein neuer Netzteilnehmer durch seinen Beitritt für alle anderen Netzmitglieder auslöst. Denn ein neues Netzmitglied führt zu einer Vergrößerung des Netzwerkes, wovon alle Netzmitglieder profitieren. Dabei wird die Veränderung des Nutzens der Netzmitglieder nicht im Marktpreis abgebildet. ${ }^{15}$ Die Netzmitgliedschaft erfullt die Eigenschaft eines Clubgutes. Ist der Konsument erst einmal Mitglied im Netz, kann er von der Netznutzung nicht ausgeschlossen werden, und der Nutzen, den alle Teilnehmer aus seinem Beitritt haben, bzw. der Nutzen, den alle aus der Netzgröße haben, rivalisiert nicht.

Ein Konsument zieht einen positiven Nutzen daraus, wenn sich der Umfang eines Netzes vergrößert. Eine Netzvergrößerung ist zum einen auf eben beschriebene

13 In einem der ersten Artikel über Netzeffekte von Katz/Shapiro (1985) behaupteten die Autoren, daß Netzeffekten Netzexternalitäten entsprăchen und unternahmen deswegen keine sprachliche Differenzierung. Da aber nicht immer Netzeffekte auch Externalităten implizieren, sollten die Begriffe nicht synonym verwendet werden. Das, was sie als indirekte Netzexternalităt (S. 424) bezeichneten, kann durch Preisveränderungen auf anderen Märkten ausgelöst werden, kritisieren Liebowitz/Margolis (1994), S. 139; (1995), S. 3 ff. Das, was sie als direkte Netzexternalităt bezeichneten, ist teilweise internalisierbar, und deswegen liegt nicht grundsätzlich ein Marktversagen vor. Vgl. Liebowitz/Margolis (1995), S. 13. In spăteren Arbeiten von Katz/Shapiro (1994) nahmen sie die Kritik an dem Begriff Netzexternalität auf und verwendeten stattdessen einheitlich den Begriff der Netzwerkeffekte.

14 Zum Beispiel zeigen Chou/Shy (1996) auf, daß Netzeffekte auch ohne Netzexternalitäten entstehen können.

15 Diese Netzexternalität kann auch als technologische Externalität bezeichnet werden - eine Verănderung der Konsum- oder Produktionsfunktion ohne eine Preisverănderung. Vgl. Liebowitz/Margolis (1994), S. 137. Dieser Begriff bezeichnet die Gruppe an Externalitäten, die bislang im Zusammenhang mit Marktversagen und Möglichkeiten der Internalisierung untersucht wurden. Einige Autoren sprechen bei dem Auftreten von Netzexternalitäten oder bei einer uberproportionalen Nutzensteigerung aufgrund einer linear steigenden Verwendung von Netzprodukten von nachfrageseitigen steigenden Skalenertrăgen, weil eine linear steigende Verwendung von Netzeffektgütern zu einer überproportionalen Nutzensteigerung führt. Vgl. dazu Katz/Shapiro (1986), S. 824; Wiese (1990), S. 92; Besen/Farrell (1994), S. 118. Der Begriff der ,econonies of scale' trifft die Problematik nicht ganz, weil vom Nutzenzuwachs alle Konsumenten profitieren. Vgl. Köster (1999), S. 9 f. Bei dem Nutzenzuwachs handelt es sich vielmehr um einen externen Effekt. 
Netzexternalitäten zurückzuführen, zum anderen auf pekuniäre externe Effekte ${ }^{16}$, also Preiseffekte, die sich bei einem wohlfunktionierenden Marktmechanismus aufgrund zusätzlicher Nachfrage ergeben. Der Term ,Netzexternalität ${ }^{\star}$ umfaßt nicht beide Effekte. Der Begriff soll nur verwendet werden, wenn in einem Gleichgewicht unausgenutzte Gewinne bedingt durch eine Netzwerkteilnahme ausgewiesen werden. ${ }^{17}$ Der Gebrauch eines Gutes löst dann eine Netzexternalität aus, wenn der Wert eines Gutes für einen Nutzer steigt, sobald ein anderer Konsument sich dem Netz anschließt und durch seine Teilnahme das Netz vergrößert. Der einzelne Nutzer wägt bei der Anschaffung des Gutes seinen individuellen Nutzen gegenüber seinen individuellen Kosten ab. Die Nutzensteigerung für alle anderen Netzteilnehmer durch seinen Beitritt in das Netz berücksichtigt er nicht in seinem Kalkül. Es kommt zu einer Netzwerkgröße, die wohlfahrtsökonomisch zu klein ist aufgrund nicht internalisierter Netzwerkexternalitäten.

Die Beschränkung der Definition des Externalitätenbegriffs auf ein Marktgleichgewicht schließt monetäre Effekte aus. Der Vorteil dieser Definition ist, daß Netzexternalitäten dem allgemeinen Verständnis über externe Effekte als Beispiel für Marktversagen entsprechen.

\subsubsection{MÖGLICHKEITEN DER INTERNALISIERUNG VON NETZ- EXTERNALITÄTEN}

Da das Bestehen von Netzexternalitäten ein Marktversagen impliziert, drängt sich die Frage auf, wie man sie internalisieren kann. Verfügen die Marktteilnehmer über vollständige Information bezüglich ihrer eigenen Präferenzen und die aller anderen Teilnehmer, dann könnten sich die Nutzer untereinander koordinieren, so daß ein Netzwerkeffekt nicht mehr zwangsläufig eine Netzexternalität auslösen müßte. Netzexternalitäten existieren demnach bei unvollständigen Informationen über die individuellen Zahlungsbereitschaften, nicht rationaler Erwartungsbildung und dem Bestehen von Koordinierungskosten, die in der Realität in der Regel vorherrschen. Aus diesem Grund kann eine Internalisierung von Netzexternalitäten nur teilweise möglich sein. Hierzu sollen einige Lösungsvorschläge gemacht werden:

1. Internalisierung von Externalitäten durch eine geeignete Vertragsgestaltung

\footnotetext{
16 Pekuniäre Externalităten beruhen auf Preisveränderungen, die zu einer Einkommensumverteilung zwischen Anbietern und Nachfragern führen, wobei Nutzen- und Produktionsfunktionen unverändert bleiben. Vgl. Liebowitz/Margolis (1994), S. 137 f.

17 Vgl. Liebowitz/Margolis (1994), S. 135.
} 
Ineffizienzen bedingt durch Netzwerkexternalitäten können deutlich reduziert werden, wenn betroffenen Marktteilnehmern freie Vertragswahl und -gestaltung zugestanden wird. ${ }^{18}$

2. Standardsetzung stellt das Entstehen von Netzexternalităten ein.

Netzexternalităten fallen dann nicht mehr an, wenn alle potentiellen Teilnehmer erfaßt sind, also keiner mehr ohne Netzzugehörigkeit ist. Dies kann am ehesten erreicht werden, indem ein Netz möglichst viele bzw. alle möglichen Teilnehmer umfaßt, d.h. entweder, indem Netze verschiedener Betreiber miteinander kompatibel gemacht werden oder aber es nur einen Netzanbieter gibt. In beiden Fällen kommt es zu einer Standardisierung. Ein Standard kann sich also sowohl aufgrund von Kompatibilität als auch von Inkompatibilität komplementärer Produkte zu dem Netzwerkgut des kompetitiven Betreibers ergeben.

\subsubsection{KOMPATIBLE STANDARDS}

Bei horizontalen Netzprodukten ermöglicht Standardsetzung durch Kompatibilităt von verschiedenen Netzwerken (horizontale Kompatibilităt) Nutzern, zwischen den Netzen ohne zusätzliche Kosten beliebig zu switchen. D.h. Netzeffekte können übertragen werden. Man kann auch von Zusammenschaltung sowohl von miteinander im Wettbewerb stehenden als auch komplementären Netzwerken sprechen. Die Tatsache, daß verschiedene Netzangehörige das andere Netz mitnutzen können, erhöht die Netzgröße aller Anbieter. Außerdem wird durch die Kompatibilität das Risiko reduziert, sich auf ein Netz festzulegen, wodurch wiederum neue Nachfrager angezogen werden. ${ }^{19}$

Bei vertikalen Netzprodukten bedeutet Kompatibilität, daß Konsumenten Komponenten unterschiedlicher Systeme frei kombinieren können. Kompatibilităt kann durch Absprache zwischen Anbietern erfolgen, so daß Komplementärprodukte zu den Netzwerkgütern der verschiedenen Anbieter passen (vertikale Kompatibilităt), oder aber kleinere Anbieter machen ihre Komplementärprodukte mit Hilfe von Konvertern oder Adaptern zu dem Netzwerkgut des Marktfuhrers (GatewayTechnologie) kompatibel.

Es ist anzunehmen, daß die Kompatibilitätsanreize in sehr jungen Produktlebenszyklusphasen bzw. in kleinen noch wachsenden Netzwerken sehr hoch sind. ${ }^{20} \mathrm{Ge}$ stalten Unternehmen ihre Produkte inkompatibel bzw. stutzen sich die Produkte auf inkompatible Systeme, dann stehen sie schon in einem sehr fruhen Stadium ih-

18 Thum (1994 und 1995, S. 88-106) analysiert Updateverträge, Serviceverträge sowie Wettbewerb der Kontrakte.

19 Auf die Zusammenschaltung von Netzen wird in Kap. 4.2 explizit eingegangen.

20 Vgl. hierzu Katz/Shapiro (1986b). 
rer industriellen Entwicklung unter enormem Wettbewerbsdruck bei der Anstrengung, eine installierte Basis aufzubauen. Die Unternehmen benutzen Produktkompatibilität in der frühen Produktlebenszyklusphase, um den Wettbewerb untereinander zu reduzieren. Kein Unternehmen ist dann gezwungen, möglichst schnell eine kritische Masse zu erreichen. Es werden folglich keine oder nur wenige Anbieter aus dem Markt ausscheiden. Auf diesem Weg wird schneller eine Netzvergrößerung erreicht. Betreiber kompatibler Systeme sowie auch die Kunden profitieren von den Netzeffekten, und Netzexternalitäten werden teilweise reduziert. Die Erhöhung der Profite der Netzanbieter aufgrund des reduzierten Wettbewerbs steigert wiederum Kompatibilitätsanreize.

In den folgenden Perioden erhöht Kompatibilität den Wettbewerb, weil es zum einen mehrere Anbieter gibt, da in der Anfangsphase keine Unternehmen aus dem Markt ausgeschieden sind. Zum anderen ermöglicht Kompatibilität den Nutzern das Hin- und Herswitchen zwischen den verschiedenen Dienstleistungsangeboten unterschiedlicher Anbieter ohne zusätzliche Kosten. Kompatibilitätsanreize gegenüber neuen potentiellen Wettbewerbern lassen dann nach.

Selbst für einen First-Mover für horizontale wie vertikale Netzprodukte kann es interessant sein, Kompatibilitätsstandards zu unterstützen und damit den Eintritt von Wettbewerbern in den Markt z.B. bei Technologien zu ermöglichen, bei denen die Realisierung von Netzeffekten einen so hohen Output erfordert, den der Monopolist allein gar nicht produzieren kann. ${ }^{21}$ Er bietet einer Zahl an Unternehmen die Lizenz, seine Technologie zu nutzen und ermöglicht ihnen damit einen Markteintritt und erzielt gleichzeitig eine Auflösung der Marktkonzentration. Der First-Mover hat an einer weniger konzentrierten Marktstruktur Interesse, wenn dadurch ein Aufbau des Netzes gewährleistet ist. D.h. in diesem Fall wirkt das Bestehen von Netzeffekten im Hinblick auf die Marktstruktur konzentrationssenkend. Neben dem Ausschöpfen der Netzwerkeffekte und der Netzexternalitäten aufgrund des erhöhten Outputs kann es zudem zu einem Wettbewerbseffekt aufgrund der gestiegenen Zahl an Anbietern kommen. ${ }^{22}$

Auf der anderen Seite kann es durch Kompatibilität zu einer höheren Anbieterkonzentration kommen, sofern Kompatibilität die Möglichkeit der Unternehmen zur Produktdifferenzierung verringert. Verringerte Produktdifferenzierung erhöht den Wettbewerb und senkt Anreize zu einem Markteintritt neuer Unternehmen aufgrund schlechterer Ertragsaussichten für die Newcomer. ${ }^{23}$

Es gibt verschiedene Möglichkeiten bei der Durchsetzung von Kompatibilitätsstandards. Die eine ist, daß es zu Verhandlungen bzw. zu Absprachen zwischen

21 Vgl. Economides (1996b), Farrell/Gallini (1988).

22 Allerdings muß hier kritisch angemerkt werden, daß diese Situation Absprachen zwischen den Anbietern begünstigt. Vgl. dazu Kap. 5.3.

23 Vgl. Matutes/Padilla (1994). 
den Akteuren uber technische Voraussetzungen kommt. Das Problem hierbei ist, daß die Anreize für eine Standardisierung zwischen den Akteuren je nach Unternehmens- und Netzgröße divergieren.

Die zweite Möglichkeit besteht darin, durch den Staat in Form von Institutionen und Behörden Kompatibilitätsstandards vorzuschreiben. Auch diese Möglichkeit beinhaltet viele Probleme. Neben den Informationsdefiziten, die eine Behörde aufgrund fehlender Marktnähe immer hat, entsteht zudem noch das Problem des rent-seeking, also Anreize von Unternehmen, die Behörden zu beeinflussen, sowie die Gefahr der Überregulierung. ${ }^{24}$

In der Praxis hat man in der europäischen Telekommunikationsbranche eine Mischform aus beiden Möglichkeiten umgesetzt. Die Normierungsaufgaben werden an das ETSI ubertragen, eine Standardisierungsinstitution, die sowohl aus Unternehmensorganisationen sowie öffentlichen Normierungsinstitutionen besteht. Das ETSI setzt sich zusammen aus Vertretern von Netzbetreibern, Geräteherstellem, Diensteanbietern, Verbänden, Benutzerorganisationen, Forschungsinstituten und ehemals staatlichen Telekommunikationsgesellschaften. ${ }^{25}$ Das ETSI geht über die rein freiwillige Kooperation hinaus, hat aber geringere rent-seeking Anreize, weil der Anteil aus der Industrie ubergewichtig vertreten ist. Ihre Aufgabe ist das gemeinsame Festlegen und Umsetzen von Standards wie das $\mathrm{OSI}^{26}$ für einen offenen Netzzugang und Einrichtungen von Brulcken.

\subsubsection{INKOMPATIBLE STANDARDS}

Es gibt ebenso die Möglichkeit einer Standardisierung bei inkompatiblen Netzwerken. Netzbetreiber bedienen ihre Teilnetze als Monopolisten, stehen aber im Wettbewerb zueinander in bezug auf die Gewinnung von Neukunden. Die Folge kann ruinöse Konkurrenz sein. Es wird eine industrieweite Standardisierung seitens eines Anbieters durchgesetzt, indem nur ein System überlebt. Auch so können Netzwerkexternalitäten bestmöglich ausgeschöpft werden. Als Beispiel sei hier der Wettbewerb zwischen VHS (Video Home System) der Firma JVC und dem Betamax-System der Firma Sony genannt. Beide Systeme waren zueinander inkompatibel. Beim Aufbau einer installierten Basis hat sich ein System - das VHS - durchgesetzt, obwohl das Betamax-System als erstes auf dem Markt war und einen first-mover Vorteil hatte. ${ }^{27}$ Dies ist auch ein gutes Beispiel dafür, daß

24 Vgl. hierzu auch Kap. 6.

25 Vgl. Klodt u.a. (1995), S. 122, Monopolkommission (1991).

26 Das OSI macht Netze mit unterschiedlichen technischen Spezifikationen horizontal wie vertikal kompatibel. Vgl. Kap. 3.4.4.3.

27 Zwar war das Betamax-System in den USA zuerst auf dem Markt (1975) und hatte zunächst einen Marktanteil von $100 \%$. Dennoch hat sich das VHS auf dem Markt durchgesetzt. Für den 
das Durchsetzen eines Systems unabhängig von der anfänglichen installierten Basis sein kann. Für die Etablierung eine Standards ist die Erwartungsbildung der Konsumenten über die Wettbewerbsfähigkeit eines Netzanbieters und folglich über seine zukünftige Netzgröße entscheidend.

\subsubsection{BEWERTUNG VON STANDARDS}

Ob Standardsetzung bedingt durch Kompatibilität oder durch Inkompatibilität gesamtwirtschaftlich effizienter ist, läßt sich generell nicht beantworten. Es ist nicht einsehbar, ob Unternehmen sowie eine Behörde effiziente Anreize haben, Kompatibilitätsstandards zu fördern oder nicht. Insofern kann Kompatibilität sowohl wohlfahrtssteigernd als auch wohlfahrtsmindernd sein. ${ }^{28}$ Es kann also nicht eindeutig gesagt werden, ob Kompatibilität erforderlich ist, um das Problem der Netzexternalitäten bestmöglich zu lösen. Die Durchsetzung eines Standards bei inkompatiblen Systemen, die sich durch Marktmechanismen ergibt, kann in bezug auf das Ausschöpfen von Netzexternalitäten ebenso sinnvoll sein. Desweiteren soll hier auf Probleme hingewiesen werden, die sich aus einer Standarisierung ergeben, unabhängig davon, ob sie durch Kompatibilität oder Inkompatibilität zustande gekommen ist.

a) Ein Veralten von Standards kann das Verharren in einer alten Technologie begünstigen. Standardisierungen, die durch Kompatibilität wie Inkompatibilität bewirkt worden sind, bringen das Problem mit sich, daß sie zu Ineffizienzen bei raschem technologischen Fortschritt füren können. Eine Industrie, die ihre Produkte einmal standardisiert und kompatibel gemacht hat, wird wegen Koordinationsproblemen nur ungern einen neueren Standard einführen. Die Erträge aus der alten Standardisierung überwiegen zum Zeitpunkt der Entscheidung die Kosten, die bei der Einführung eines neuen Standards entstehen. Ursachen solcher Entwicklungen können Pfadabhängigkeiten sein. Durch kurzfristig richtige Entscheidungen entsteht eine ökonomische Pfadabhängigkeit, die langfristig zu einer nicht wünschenswerten Marktlösung führt. ${ }^{29}$ Es besteht das Risiko, daß eine Technologie veraltet, die sich als Standard einmal durchgesetzt hat, und es zu einem LockIn kommt. Neuen technischen Systemen fallt es schwerer, eine kritische Masse zu

Durchbruch entscheidend war, da $B$ die maximale Spieldauer der VHS-Videokassetten über zwei Stunden betrug, während die Spieldauer der Betamax-Kassetten immer darunter lag. Die Kassetten waren kleiner und hatten deswegen entsprechend weniger Magnetband. Die Spieldauer von zwei Stunden - Spielfilme haben in der Regel eine Spielzeit von 90 bis 120 Minuten - erleichterte die Entstehung des Komplementärmarktes für bespielte Videokassetten und Videotheken. Vgl. Kleinemeyer (1997), S. 47 sowie Katz/Shapiro (1986b), S. 825.

28 Vgl. Klodt (1995), S. $123 \mathrm{f}$.

29 Vgl. Arthur (1988a,b). Zur Ursachen von Pfadabhängigkeiten vgl. Blankart/Knieps (1991). 
erreichen und sich am Markt zu behaupten. ${ }^{30}$ David (1985) spricht von einer Ineffizienzhypothese der marktlichen Standardisierung, die er mit Hilfe einer Fallstudie über die Geschichte der Etablierung des Qwerty-Tastaturstandards versuchte zu verifizieren. ${ }^{31}$

b) Der durchgesetzte Standard kann ein technisch schlecht entwickelter sein. ${ }^{32}$ Beispielsweise wird von den Betamax-Kassetten behauptet, daß sie technologisch anspruchsvoller seien als die VHS-Kassetten. Dennoch hat sich das VHS-System durchgesetzt, weil es offenbar bedienungsfreundlicher ist. Ein technologisch schlechteres Produkt muß folglich nicht immer das ökonomisch Schlechtere sein.

Im folgenden Kapitel sollen sowohl Netzeffekte als auch Netzexternalitäten für horizontale Netzprodukte, insbesondere für den Markt für Telekommunikationsdienste formalisiert werden. Außerdem soll auf horizontale Kompatibilităt in Form einer Zusammenschaltung von Netzen sowie einer teilweisen Internalisierung externer Effekte eingegangen werden.

30 Vgl. dazu Arthur (1989), David (1985), Farrell/Saloner (1985), die in diesem Zusammenhang von, excess inertia' sprechen.

31 Arthur (1989) lieferte den modelltheoretischen Unterbau für Davids Ineffizienzhypothese. Qwerty steht für die Buchstabenfolge der oberen Tastenreihen bei Schreibmaschinen und Pcs. C.P. Sholes erfand 1867 die mechanische Schreibmaschine und die Qwerty-Tastatur mit dem Ziel, die Schreibgeschwindigkeit absichtlich zu reduzieren, damit sich die Buchstabentypen möglichst wenig ineinander verhaken. Schreibmaschinen mit einer neuen Buchstabenanordnung, die die Geschwindigkeit erhöhten, nachdem es eine technische Verbesserung in bezug auf die Mechanik der Schreibmaschinentastaturen gab, konnten sich nicht durchsetzen, weil 1. der Wert einer Schreibmaschine an der Verfügbarkeit von Schreibkräften, die mit der jeweiligen Schreibmaschine und ihrer Tastaturanordnung umgehen konnten, gemessen wurde und 2. das Lernen und Umlernen der Schreibkrăfte auf eine neue Tastatur mit irreversiblen Kosten verbunden war. 1932 entstand die Dvorak- Tastatur von A. Dvorak und W.L. Dealey, die sich fulr ihr Geschwindigkeitsschreiben auszeichnete und schneller erlernbar war. Diese beiden Eigenschaften hat Dvorak anhand von empirischen Versuchen nachgewiesen. Dennoch konnte sie sich nicht gegenuber der Qwerty-Tastatur behaupten. Liebowitz/Margolis (1990) kritiserten die Verifikation der Ineffizienzhypothese an der Qwerty-Fallstudie. Erstens zweifelten sie die Aussagekraft der empirischen Tests an. Die empririschen Grundlagen seien zu dürftig bzw. das verwendete empirische Material fragwlirdig. Zweitens kritisierten sie den Ansatz, einen Standard als öffentliches Gut zu bezeichnen. Stattdessen gibt es einen Eigentümer eines Standards. Die Berilcksichtigung von Eigentumsrechten ermöglichen die Bildung von vertraglichen Arrangements zur Internalisierung von Netzexternalitäten sowie Maßnahmen zu einem Standardwechsel, die David (1985) außer Acht gelassen habe. Vgl. Wey (1999), S. 57.

32 Farrell/Saloner (1985) haben Bedingungen entwickelt, unter denen Benutzer dem inferioren Adoptionspfad folgen (,excess monumentum'). 
Angenommen, $u_{B}$ repräsentiert den Nutzen, den ein Konsument daraus hat, bei einer Telefongesellschaft $B$ angemeldet und an ein Netz angeschlossen zu sein, dann ließe sich folgende Nutzenfunktion formulieren: ${ }^{33}$

$$
u_{B}=e+b f\left(q_{B}\right) \text {. }
$$

$e$ entspricht dem Stand-Alone-Nutzen aus dem jeweiligen Netzwerkgut. Er ergibt sich aus dem Kauf des Gutes, in diesem Fall aus dem Besitz eines Teilnehmeranschlusses und eines Endgerätes. Dieser kann individuell unterschiedlich sein. Hier wird der Einfachheit davon ausgegangen, daß alle Kunden einen gleichen Nutzen aus einem Telefonanschluß ziehen.

$b f\left(q_{B}\right)$ bildet den Derivativnutzen ab, den ein Konsument bei der Teilnahme an einem Netzwerk $B$ hat. Dabei stellt $b$ die Bewertung des Netzeffektes dar und soll hier positiv sein $(b>0)$. Hierbei wird angenommen, daß der Nutzen aus dem Netzeffekt für alle Teilnehmer gleich ist. ${ }^{34}$

In der oben spezifizierten Nutzenfunktion wird von einem ausschließlich positiven Zusammenhang zwischen Nutzen und Netzgröße ausgegangen. ${ }^{35}$ Der Nutzen kann aber auch bei zu vielen Netzteilnehmern mit jedem weiteren Netzteilnehmer abnehmen. Dann ist das Netz überfüllt. Es kommt zu Warteschleifen, Datenstaus und im schlimmsten Fall zu Datenverlust. In diesem Fall würde jeder weitere Netzteilnehmer negative Netzexternalitäten verursachen. Dieser Fall soll hier auBer Acht gelassen werden.

Hier wird formal unterstellt, daß die Wirkung des Netzeffektes additiv wirkt. Der Nutzen aus dem Netzeffekt ergibt sich aus der tatsächlichen Zahl der Netzmitglieder $q_{B}$ des Netzwerkes $B$, wobei hier nur die Zahl der Anschlüsse erfaßt wird. ${ }^{36}$ Der Wert eines Netzeffektgutes und damit der individuelle Nutzen jedes Netzteilnehmers steigt mit der zunehmenden Anwenderzahl. Die Netzgröße eines Netz-

33 In Anlehnung an Katz/Shapiro (1985); Farrell/Saloner (1985, 1986b). Vgl. hierzu auch Kap. 5.3.2.2.

34 Die Annahme homogener Präfernezen wird z.B. von Katz/Shapiro (1985), S. 426, getroffen. Baake (1996) untersucht allerdings auch heterogene Präferenzen.

35 Katz/Shapiro $(1985,1986 b)$ sowie Farrell/Saloner $(1985,1986 a)$ haben in ihren richtungsweisenden Arbeiten die positive Beziehung zwischen Nutzenniveau eines Konsumenten und der Netzwerkgröße als exogen vorgegeben postuliert. Economides (1996) bezeichnet diesen Modellansatz als Makroansatz.

36 Katz/Shapiro (1994) berücksichtigen die erwartete Zahl an zukünftigen Netzmitgliedern bzw. die erwartete Netzwerkgröße. Bei Konsumenten fließen bei der Kaufentscheidung Erwartungen über die zukünftig existierende Netzgröße mit ein. Dabei kommt es darauf an, welche Erwartungsbildungskonzepte unterstellt werden (autoregressiv oder rational), da sie Einfluß auf das kompetitive Gleichgewicht ausüben. 
werkes $B$ ergibt sich aus der Summe aller Teilnehmeranschlüsse in dem betreffenden Netzwerk.

$f\left(q_{B}\right)$ entspricht den Kombinationsmöglichkeiten innerhalb eines Netzes, mit denen der Nutzer mit anderen Teilnehmern in Kommunikation treten kann, und ist eine differenzierbare Funktion, wobei $f(0)=0$ und $f\left(q_{B}\right)>0$ furr $q_{B}>0$. In dem Netz $B$ ergeben sich mit jedem weiteren Teilnehmer $q_{B}\left(q_{B}-1\right) / 2$ Kombinationsmöglichkeiten, sofern ein bidirektionales Netz bzw. wechselseitiger Leitungsverkehr vorliegt. Demnach ergibt sich folgender Grenznutzen:

$$
\begin{aligned}
& \frac{\partial u_{B}}{\partial q_{B}}=b\left(q_{B}-\frac{1}{2}\right)>0, \\
& \frac{\partial^{2} u_{B}}{\partial q_{B}}=b \quad>0 .
\end{aligned}
$$

Ist das Netz unidirektional bzw. liegt gerichteter Leitungsverkehr vor, entspricht die Zahl an möglichen Kombinationen $f\left(q_{B}\right)=q_{B}\left(q_{B}-1\right)$, so daß sich dann ein folgender Grenznutzen ergibt:

$$
\begin{aligned}
& \frac{\partial u_{B}}{\partial q_{B}}=b\left(2 q_{B}-1\right)>0, \\
& \frac{\partial^{2} u_{B}}{\partial q_{B}{ }^{2}}=2 b>0 .
\end{aligned}
$$

Der Nutzen bzw. der Grenznutzen des Netzanschlusses erhöht sich sowohl bei wechselseitigem als auch gerichteten Leitungsverkehr mit der Zahl der Netzteilnehmer. Ein einzelner Teilnehmer wird bereit sein, mehr für den Anschluß zu zahlen, je größer das Netzwerk ist, weil die individuelle Zahlungsbereitschaft mit der Netzgröße steigt. Die erhöhte Zahlungsbereitschaft ist auf eine monetäre Bewertung des auftretenden Netzeffektes seitens der Teilnehmer zurlickzufuhren. Es liegt also eine strategische Entscheidungssituation vor, da das Verhalten eines Nachfragers vom Verhalten aller anderen Netzteilnehmer bestimmt ist. Nun löst aber der Beitritt eines neuen Teilnehmers in das Netz jenen positiven externen Effekt fur die bisherigen Mitglieder aus, den der Beitretende nicht in seinem individuellen Kosten-Nutzenkalkül berlucksichtigt und der sich im Beitrittspreis nicht widerspiegelt. Folglich wird eine zu kleine Netzgröße bereitgestellt. Um die durch den Netzbeitritt entstehende Netzexternalität formal zu verdeutlichen, muß ein gesamtgesellschaftlicher Nutzen $G U$ des Netzwerkes $B$ ermittelt werden. Für die Ermittlung des Gesamtnutzens wird unterstellt, daß alle Teilnehmer des Netzwerkes $B$ einen identischen Netzwerknutzen haben und daß sich die einzelnen Nut- 
zen aufsummieren lassen. ${ }^{37}$ Der Gesamtnutzen ist abhängig von der Anzahl der Netzwerkteilnehmer:

$$
G U\left(q_{B}\right)=q_{B} e+q_{B} b f\left(q_{B}\right) \quad \text { für } q_{B}>0 .
$$

In der Abbildung 4.2-1 ist der Nutzen aller Teilnehmer aus dem Netzwerk abgebildet, also der Nutzen aus dem Beitritt der anderen. Es besteht ein positiver $\mathrm{Zu}-$ sammenhang zwischen der Netzwerkgröße und dem Nutzen aller aus dem Netzwerk. Hat das Netzwerk die Größe $q$, dann realisieren alle Netzteilnehmer ein Gesamtnutzenniveau von $G U_{q}$. Tritt ein weiterer Teilnehmer in das Netzwerk ein, erhöht sich das Nutzenniveau aller Teilnehmer auf $G U_{q+1}$. Der externe Effekt ergibt sich graphisch aus der Fläche zwischen $G U_{q}$ und $G U_{q+1}$. Formal läßt sich der externe Effekt (EE) folgendermaßen formulieren:

$$
E E=G U_{q+1}(q+1)-G U_{q} q-\int_{q}^{q+1} G U(q) .
$$

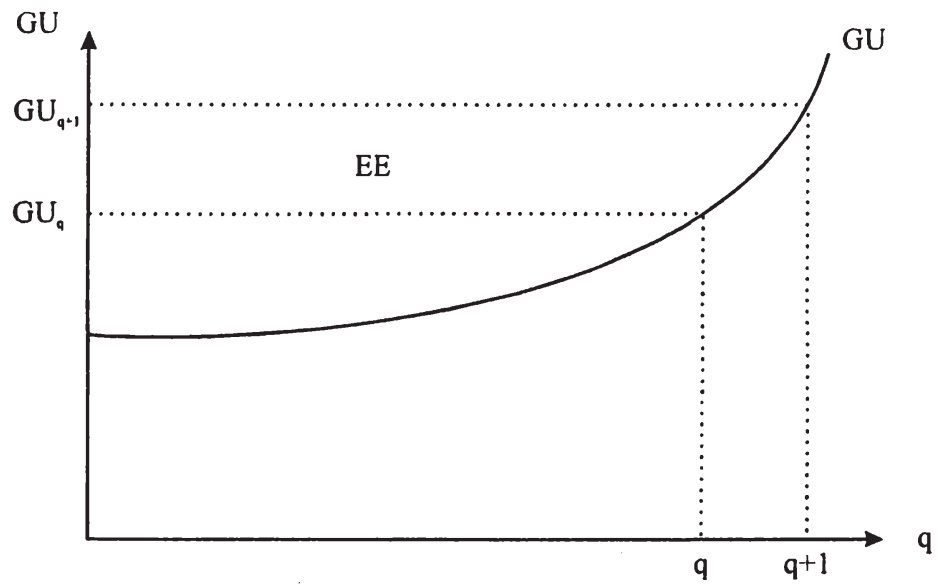

\section{Abbildung 4.2-1: Darstellung der Höhe von Netzexternalităten}

Gelingt eine vollständige Netzabdeckung der gesamten Bevölkerung in bezug auf Telekommunikationsdienste, dann wären alle Netzexternalitäten internalisiert.

37 D.h. hier wird implizit interpersonelle Vergleichbarkeit und kardinale Meßbarkeit der Nutzenniveaus unterstellt. Gewiß ist eine solche Annahme als äußerst kritisch zu beurteilen. 
Dies sagt zunächst nichts über die bestmögliche Marktstruktur aus. Denn eine vollståndige Netzabdeckung kann sowohl von einem Monopol erreicht werden als auch von mehreren Anbietern, sofern sie ihre Netze zuammenschalten (horizontale Kompatibilität). Eine vollständige Netzabdeckung ist aber nicht unbedingt wohlfahrtsverbessernd. Für die Kunden ist sie in jedem Fall von Vorteil und erhöht die Konsumentenrenten. Für die Anbieter dagegen ist eine vollständige Netzabdeckung als vorgegebenes Ziel nicht unbedingt vorteilhaft, da dies besonders in ländlichen Regionen zu einer Kostenexplosion führen kann. Der Grenznutzen aus einer Netzvergrößerung muß folglich mit den anfallenden Grenzkosten verglichen werden, um eine Aussage hinsichtlich der Auswirkungen auf die gesamtgesellschaftliche Wohlfahrt machen zu können. In Deutschland ist allerdings eine vollständige Netzabdeckung noch vor der Liberalisierung unter einem öffentlichen Monopol im Rahmen der gesetzlich verpflichtenden Universaldienstverpflichtung $^{38}$ aus sozialpolitischen Gründen erfolgt.

Zusammenschaltung $^{39}$ von Netzen unterschiedlicher Betreiber ist eine weitere Möglichkeit, Netzexternalităten zu internalisieren und kann generell aus ökonomischer Perspektive befürwortet werden, weil es sowohl die Konsumentenrenten als auch die Produzentenrenten erhöht. Die Konsumenten profitieren von einer $\mathrm{Zu}$ sammenschaltung, weil sie sich wie eine Netzvergrößerung auswirkt. Zum einen erhöht sich die Zahl der Kombinationsmöglichkeiten bei zusammengeschalteten Netzen; $f\left(q_{1}+q_{2}\right)>f\left(q_{1}\right)+f\left(q_{2}\right)$, d.h. Netzeffekte werden ausgeschöpft. Zum anderen werden Netzexternalitäten teilweise internalisiert. Für die Netzbetreiber kann eine Zusammenschaltung von Vorteil sein, weil die Nachfrage nach durchzuleitenden Diensteinheiten an die einzelnen Teilnehmeranschlüsse steigt (Gespräche aus dem anderen Netz) und damit auch der Output steigt. Konkurrierende Betreiber im Teilnehmeranschlußbereich monopolisieren den jeweiligen Teilnehmeranschluß, so daß ohne Zusammenschaltung keine Kommunikation zwischen Teilnehmern unterschiedlicher Netzbetreiber möglich wäre. In Deutschland sind Betreiber von Telekommunikationsnetzen, die substitutiv sowie komplementär zueinander stehen, zu einer Zusammenschaltung gesetzlich angehalten.

Die Forderung nach Zusammenschaltung konkurrierender Telekommunikationsnetze kann als ökonomische Rahmenbedingung verstanden werden, die sowohl für alle Betreiber als auch für alle betroffenen Kunden von Vorteil ist und sich damit wohlfahrtsverbessernd auswirkt. ${ }^{40}$

38 Vgl. dazu Kap. 2.4.5.

39 Im Rahmen einer Zusammenschaltung von Netzen unterschiedlicher Betreiber müssen die Netze horizontal kompatibel zueinander gemacht werden; d.h. hier geht es um die physikalische Etablierung einer Hardware-Schnittstelle.

40

Vgl. dazu Kap. 2.4.3. 
Sarah Eliza Schniewindt - 978-3-631-75130-5 Downloaded from PubFactory at 01/11/2019 07:57:00AM via free access 
Im folgenden Kapitel soll sich ausfuhrlich dem Netzzugang (One-Way-Access) sowie der Zusammenschaltung von Netzen bei einem Two-Way-Access gewidmet werden.

Der Netzzugang stellt das zentrale Problem bei der Einfuhrung von Wettbewerb in den Telekommunikationsmarkt bei einem One-Way-Access dar, weil erstens die Unterteilung in vorgelagerte und nachgelagerte Märkte existiert. Dabei ist die Funktionsfähigkeit des Marktes für Telekommunikationsdienste von vorgelagerten Märkten abhängig.' Zweitens ist die deutsche Telekommunikationsbranche davon geprägt, daß es vor der Liberalisierung 1998 ein öffentliches Monopol gab, das alle telekommunikationsspezifische Märkte bediente. Dieser Betreiber ist als Incumbent nach wie vor auf vor- und nachgelagerten Märkten präsent. Auf den vorgelagerten Märkten ist er in Teilen noch marktbeherrschend oder sogar nach wie vor der einzige Anbieter. Dies trifft vor allem auf den Teilnehmeranschlußbereich zu. Hieraus ergibt sich die Gefahr des Mißbrauchs des Netzzugangs als Markteintrittsbarriere sowohl für Wettbewerber, die nur auf den nachgelagerten Markt eintreten wollen (Diensteanbieter), als auch fur substitutive Wettbewerber. $\mathrm{Zu}$ den substitutiven Anbietern, deren Funktionsfähigkeit einen Zugang zum Netz des Incumbents zwingend erfordert, gehören zum einen Anbieter von Internet Telefonie, da sie in der Regel Anschlüsse im Teilnehmeranschlußbereich anmieten müssen ${ }^{2}$, sowie alternative Anschlußbetreiber, deren Netz sich noch im Aufbau befindet. Dies trifft insbesondere für funkgestützte Anschlußtechnologien zu wie WLL oder Mobilfunk ${ }^{3}$, weil sie in ihrer Netzaufbauphase Zugang zur Infrastruktur des Incumbents benötigen. Aus diesem Grund ist der Netzzugang sowie eine Entgeltregulierung gesetzlich verankert. ${ }^{4} \mathrm{Im}$ weiteren Verlauf soll auf die Problematik bei der Bestimmung von Zugangsentgelten eingegangen werden. Desweiteren soll ebenso der Two-Way-Access näher vorgestellt werden. Er soll hier auf eine Marktsituation bezogen werden, in der insbesondere im Teilnehmeranschlußbereich substitutive Netzbetreiber im Wettbewerb zueinander stehen. $\mathrm{Zu}$ den substitutiven Anbietern zählen Betreiber von umgerüsteten TV Kabeln sowie PLC Betreiber, die, wenn beiden eine Umrüstung erst einmal gelingt, in kurzer Zeit sehr viele Kunden anschließen können, so daß sie schnell einen hohen Marktanteil erzielen können, weil sie nicht erst ein Netz im Teilnehmeranschlußbereich aufbauen müssen. ${ }^{5}$ Deren Zusammenschaltung von Netzen ist ökono-

\footnotetext{
Vgl. dazu Kap. 2.1, insbesondere Abbildung 2.1-3.

Vgl. hierzu Kap. 3.4.1.3 sowie 3.4.4.

Vgl. hierzu Kap. 3.4.2.1 sowie 3.4.2.2.

Vgl. dazu Kap. 2.4.2.

Vgl. zu einer genauen Beschreibung der Technologien Kap. 3.4.1.1 sowie Kap. 3.4.1.2.
} 
misch von Vorteil und gesetzlich festgelegt. ${ }^{6}$ Zudem liegt eine Regulierung von Zusammenschaltungsentgelten vor. Im weiteren Verlauf sollen die Gründe für eine gesetzliche Entgeltvorgabe hinterfragt werden.

\subsection{Allgemeine Darstellung von Preisregeln}

Im Zuge der eingangs formulierten Problematik bei der Regelung des Zugangs zum Netz des Incumbents soll eine Auswahl an Preisregeln vorgestellt werden, die in Kapitel 5.2 zunächst aus einem theoretischen Blickwinkel beleuchtet und schließlich in Kapitel 5.4 in bezug auf die Regulierungspraxis für die Bestimmung von Zugangs- bzw. Zusammenschaltungsentgelten angewendet werden. Es handelt sich hierbei um statische Konzepte der Einzelpreisregulierung. Die verschiedenen Preisregeln werden hinsichtlich dreier Kriterien untersucht:

- Allokative Effizienz. Sie ist dann gegeben, wenn die Summe aller Konsumenten- und Produzentenrenten maximiert ist. Das Bestimmen allokativer Effizienz unterstellt, daß Kostenfunktionen und Konsumentenpräferenzen für die Ermittlung des Endproduktpreises bekannt sind. ${ }^{7}$

- Produktive Effizienz ${ }^{8}$. Sie unterstellt die kostenminimale Produktion eines vorgegebenen Outputs. Jede Abweichung von einer kostenminimalen Produktion kann als technisch ineffizient bezeichnet werden. Es gibt mehrere Ursachen für das Vorliegen technischer Ineffizienz, z.B. das Nicht-Aussschöpfen von Größenvorteilen (Economies of Scale and Scope) oder jedwede Form an Ressourcenverschwendung wie ein zu hoher materieller und personeller Produktionsfaktoreinsatz oder zu hohe Input-Preise. ${ }^{9}$

$6 \quad$ Vgl. zur ökonomischen Betrachtung von Netzzusammenschaltung Kap. 4.2, zur gesetzlichen Regelung von Zusammenschaltung Kap. 2.4.3.

7 Vgl. Kruse (1985), S. 73 f.

8 Vgl. Kruse (1985), S. 97 ff. Alternativ werden auch folgende Begriffe verwendet: Technische Effizienz, Kosten-Effizienz, interne Effizienz.

9 Dynamische Effizienz wird in der Regel als drittes Effizienzkriterium genannt. Vgl. hierzu Kruse (1985), S. 117 ff. Es bezieht sich erstens auf Veränderungen der statischen Effizienzkriterien im Zeitablauf. Die Veränderungen können bedingt sein durch exogene Schocks auf die Nachfrage oder auf die Angebotsstruktur. Zweitens kann sich dynamische Effizienz auf eine endogene Veränderung des Effizienzoptimums beziehen, die durch Innovationen ausgelöst werden kann. Dabei kann dynamische Effizienz im Widerspruch zu allokativer oder technischer Effizienz stehen.Zum Beispiel kann ein Ergbenis in einer Periode allokativ wie produktiv ineffizient sein, hinsichtlich einer mehrperiodigen Betrachtung durchaus dynamisch effizient sein. Da hier aber nur statische Konzepte für die Ermittlung von Preisen über einen Zeitraum von einer Periode analysiert werden, sollen die Preisregeln nur hinsichtlich ihrer allokativen und technischen Effizienz überprüft werden und nicht hinsichtlich ihrer dynamischen Effizienz. Vgl. zur dynamischer Effizienz Donges/Freytag (2001), S. 121; Baumol/ Ordover (1992), S. 83. 
- Promotion von Wettbewerb. Hierbei soll eine Aussage daruber getroffen werden, inwieweit sich die Preisregeln eignen, Wettbewerb einzuführen und zu etablieren. ${ }^{10}$

\subsubsection{DIE GRENZKOSTENPREISREGEL}

In einem perfekt kompetitiven Umfeld wird ein Einproduktunternehmen seinen Preis an der Deckung der Grenzkosten orientieren. Diesem Konzept ist eine atomistische Marktstruktur und perfekte Markttransparenz unterstellt. Grenzkostenpreise werden häufig auch als ,first-best' Ergebnis bezeichnet, weil sie unter den gegebenen Annahmen ein ökonomisch effizientes Ergebnis in statischer Hinsicht in bezug auf produktive und allokative Effizienz liefern.

Es können kurzfristige wie langfristige Grenzkosten zu Grunde gelegt werden. Werden kurzfristige Grenzkosten für die Bestimmung von Preisen gewählt, bleiben getätigte Investitionen in eine Infrastruktur unberücksichtigt und die gewăhlte Betriebsgröße unveränderlich. Sie umfassen nur die variablen Stück- bzw. Einzelkosten. Kurzfristige Grenzkosten eignen sich für eine einperiodige Betrachtung. Werden langfristige Grenzkosten unterstellt, dann impliziert das eine mehrperiodige Analyse, in der Unternehmen alle Inputfaktoren varriieren, so auch die Infrastrukturinvestitionen wie die Betriebsgröße. Langfristig werden alle Kosten variabel. Die Berücksichtigung langfristiger Grenzkosten umfaßt damit alle Kostenkategorien. " In der Ordnungspolitik werden im allgemeinen in bezug auf Preisregeln langfristige Grenzkosten angewendet, da diese bei Nachfrageschwankungen stabiler sind und eine mehrperiodige Betrachtung zulassen. ${ }^{12}$

Eine Grenzkostenregel eignet sich nur unter Idealbedingungen für die Einführung von Wettbewerb. Liegen Economies of Scale und/oder durchgängig abnehmende langfristige Durchschnittskosten vor, d.h. handelt es sich bei dem betrachteten Einproduktunternehmen um ein Starkes Natürliches Monopol ${ }^{13}$, dann decken Preise, die nur die jeweiligen Grenzkosten enthalten, nicht die insgesamt anfallenden Kosten. Das Unternehmen würde dementsprechend Verluste machen. Unter diesen Bedingungen würde eine Preisfindung entlang der Grenzkosten keine ad-

10 Die Europäische Kommission (1995), S. 16, analysiert zudem noch ,Social Pricing“.

"Der Einsatz langfristiger Grenzkosten verfolgt das Ziel, die optimale Betriebsgröße bei kostenminimalem Einsatz von Inputs bestimmen zu können. Vgl. Needy (1997), S. 90 f. Sie dienen hauptsächlich als Planungsinstrument zur Beurteilung der Eignung von neuen Produktionstechnologien. Vgl. Winzer (2000), S. $111 \mathrm{f}$.

12 Vgl. Slater (1989), S. 146.

13 Zu einer genauen Definition eines Starken Natürlichen Monopols vgl. Kap. 2.3.1 sowie Abbildung 2.3.1.-1. 
äquate Lösung mehr darstellen. Handelt es sich außerdem um ein Mehrproduktunternehmen, fallen Gemeinkosten an, die in den Grenzkosten nicht berücksichtigt werden. Die Grenzkostenpreisregel wäre dann nicht das geeignete Instrument, sofern Gemeinkosten nicht mit einem zusätzlichen Aufschlag miteinbezogen würden.

\subsubsection{DIE DURCHSCHNITTSKOSTENPREISREGEL BEI EINEM STARKEN NATÜRLICHEN MONOPOL}

Bei einem Ein-Gut-Monopolisten im Sinne eines Starken Natürlichen Monopols würde, wie soeben beschrieben, die Anwendung einer Grenzkostenpreisregel zu Verlusten führen. Es stellt sich die Frage, wie hoch die Abweichung von den Grenzkosten sein muß, um letztendlich mit Nullgewinn bzw. kostendeckend zu produzieren. Der Preisaufschlag auf den Grenzkostenpreis läßt sich beispielsweise bei einem Ein-Produkt-Monopol bestimmen, indem sich die Endproduktpreise an den langfristigen Durchschnittskosten zur Deckung der Fixkosten orientieren. Sie werden auch oft als ,second-best' - Preise im Verhältnis zu den Grenzkostenpreisen bezeichnet. ${ }^{14}$ Eine Preisregel auf der Grundlage von Durchschnittskosten fuhrt nicht zu allokativer Effizienz, da es aufgrund des höheren Preises gegenüber der first-best Lösung zu einem Wohlfahrstverlust kommt. Dennoch kann sie für EinGut-Monopolisten als adäquat bezeichnet werden, um produktive Effizienz zu erzielen, da das Unternehmen sonst nicht überlebensfähig wäre. Mit dieser Preisregel kann allenfalls das Ziel der Kostendeckung zum Vorteil der Konsumenten angestrebt werden und aufgrund von Kostensubadditivität nicht die Einführung von Wettbewerb. ${ }^{15}$ Für Unternehmen, die nicht die Eigenschaften eines Natürlichen Monopols erfullen, würde die Durchschnittskostenpreisregel sowohl zu produktiver als auch zu allokativer Ineffizienz führen und Wettbewerb behindern.

\subsubsection{PREISREGEL AUF DER GRUNDLAGE VON INKREMENTELLEN KOSTEN}

Für die Bestimmung von Preisen können inkrementelle Kosten (IK) bzw. Zusatzkosten als Alternative zu den Grenzkosten herangezogen werden. Sie berücksichtigen die anfallenden Kosten für die zusätzliche Erstellung einer definierten 
Mengeneinheit eines bereits angebotenen Produktes, also eines ausgewăhlten Inkrements. Bei der Bestimmung des Inkrements ist die Größe beliebig definierbar. Zudem kann sie sich sowohl auf Produktionseinheiten als auch auf einen gesamten Output eines Produktes z. B. im Falle eines Mehrproduktunternehmens beziehen. Beide Inkremente sollen hier kurz definiert werden.

Im Falle der Bestimmung einer Produktionseinheit des Gutes $x$ lauten die Inkrementalkosten für das ausgewählte Inkrement $m, m \in H$,

$$
I K_{m}(x)=K(x)-K\left(x_{H-m}\right) .^{16}
$$

Diese Inkremental-Kosten beschreiben die anfallenden Kosten, wenn die Gutereinheit $m$ des Produktes zusätzlich zu den anderen Gütereinheiten $H-m$ produziert wird. Dabei beinhalten sie neben den variablen Stückkosten ebenso die Fixkosten. Sie ergeben sich aus der Differenz zwischen den totalen Kosten für die Herstellung des Produktes $K(x)$ und dem, was die totalen Kosten sein würden, wenn die Produktion der Einheit $m$ nicht wäre und alle anderen Einheiten unverändert blieben $K\left(x_{H-m}\right)$. Inkrementelle Kosten können mit den Grenzkosten ubereinstimmen, wenn das ausgewählte Inkrement marginal ist.

Im Falle eines Mehrproduktunternehmen können die inkrementellen Kosten eines einzelnen Produktes folgendermaßen bestimmt werden. Angenommen, das Unternehmen produziert den Output der Güter $1,2, \ldots g, n$. Dann ergeben sich die inkrementellen Kosten für den Output des Gutes $g$ aufgrund der Differenz zwischen den totalen Kosten $(K)$ für die Herstellung des Outputs $x_{1}, \ldots x_{g}, x_{n}$ und der Güter $x_{1}, \ldots, x_{n-g}$ :

$$
I K\left(x_{g}\right)=K\left(x_{1}, \ldots x_{g}, x_{n}\right)-K\left(x_{1}, \ldots x_{n-g}\right) .
$$

Der Preis für $x_{g}$ kann auf diese Weise nach den für die Produktion von $x_{g}$ anfallenden inkrementellen Kosten bestimmt werden.

Durchschnittliche inkrementelle Kosten $(D I K)$ ergeben sich, indem die inkrementellen Kosten einer zusătzlichen Gütereinheit $m$ des Produktes $x$ ins Verhältnis zum totalen Output gesetzt werden:

$$
D I K_{m}(x)=\frac{I K_{m}(x)}{x}=\frac{K(x)-K\left(x_{H-m}\right)}{x} \cdot{ }^{17}
$$

Für die Ermittlung der durchschnittlichen inkrementellen Kosten eines Produktes im Mehrproduktfall für das Gut $x_{i}$ für $i=1, \ldots g, n$ wird die Differenz der totalen Kosten $(K)$ aus der Produktion von $x_{1}, \ldots x_{g}, x_{n}$ und aus der Produktion von $x_{1}, \ldots x_{n-g}$ ermittelt und durch Output $x_{g}$ dividiert :

16 Vgl. dazu Baumol u.a. (1988), S. 67, das sich dort allerdings auf den Mehrproduktfall bezieht.

17 In Anlehung an Baumol u.a. (1988), S. 67. 


$$
\operatorname{DIK}\left(x_{g}\right)=\frac{\left\lfloor K\left(x_{1}, \ldots x_{g}, x_{n}\right)-K\left(x_{1}, \ldots, x_{n-g}\right)\right\rfloor}{x_{g}} .^{18}
$$

Dieses Konzept erinnert an durchschnittliche variable Kosten. Die durchschnittlichen inkrementellen Kosten unterscheiden sich aber darin, daß sie fixe Einzelkosten, d.h. Kosten, die sich im Falle eines Mehrproduktunternehmens dem einzelnen Produkt direkt zurechnen lassen, miteinbeziehen.

Inkrementelle Kosten sowie die durchschnittlichen inkrementellen Kosten sind im Grenzfall den Grenzkosten entsprechend allokativ wie produktiv effizient. Weiterhin spiegeln sie wegen ihrer Berücksichtigung von Fixkostenkomponenten einen langfristigen Betrachtungszeitraum wider. Langfristige inkrementelle Kosten können weiterhin hinsichtlich der Erfassung der relevanten Fixkostenkomponenten differenziert werden. Diese kann entweder an vergangenheitsorientierten Werten erfolgen oder an zukunftsorientierten, d.h. anhand von aktuellen bzw. zukünftigen Anschaffungswerten. Man spricht auch von ,Backward Looking Long Run Incremental Costs' bzw. ,Forward Looking Long Run Incremental Costs oder auch FL-LRIC bzw. ,Forward Looking Long Run Average Incremental Costs' (FL-LRAIC). Letzteres spielt in der Regulierungspraxis eine bedeutende Rolle. ${ }^{19}$ Inkrementalkosten dienen bei der Einführung von Wettbewerb als Orientierung für Preisvorgaben, die mindestens erfüllt sein müssen, damit der Incumbent ohne Verluste produzieren kann. Liegen allerdings Economies of Scale oder abnehmende langfristige Durchschnittskosten vor, können Preise, die nur die inkrementellen Kosten wiedergeben, für Mehrproduktunternehmen nicht die Gesamtkosten decken, weil sie keine Gemeinkosten erfassen, also Kosten, die von dem gesamten Unternehmen bei der Produktion mehrerer Güter verursacht werden und nicht direkt einem Endprodukt zugeordnet werden. In der Regulierungspraxis wird deswegen auf die FL-LRIC ein Aufschlag zur Abdeckung der Gemeinkosten hinzugerechnet.

\subsubsection{STAND-ALONE Kosten}

Stand-Alone Kosten $(S A K)$ sind Kosten, die entstehen würden, wenn ein neues Unternehmen für die Herstellung nur eines einzigen Produktes auf der, grünen Wiese' errichtet würde, das sonst von einem Mehrproduktunternehmen angeboten wird. Es geht also um das Herauslösen der Herstellung eines Gutes, z.B. $x_{1}$, wel-

In Anlehnung an Baumol/Sidak (1994a), S. 177.

Eine ausführlichere Beschreibung der FL-LRIC erfolgt in Kap. 5.4. 
ches sonst im Verbund mit mindestens einem anderen Produkt, hier $x_{2}$ erzeugt wird, so daß keine Skaleneffekte und keine Verbundvorteile anfallen:

$$
\operatorname{SAK}\left(x_{1}\right)=K\left(x_{1}, x_{2}\right)-I K\left(x_{2}\right) \text {. }
$$

Dann ergeben sich die Stand-Alone-Kosten des Produktes $x_{1}$, indem von den totalen Kosten $K\left(x_{1}, x_{2}\right)$, die insgesamt für die Produktion aller Güter anfallen, die inkrementellen Kosten des Gutes $x_{2}$ abgezogen werden.

Eine Preisregel auf der Grundlage von Stand-Alone-Kosten liefert ein produktiv wie allokativ ineffizientes Ergebnis im Vergleich zum Mehrproduktunternehmen, das aufgrund von Verbundvorteilen zu geringeren Kosten produzieren kann und dementsprechend $\mathrm{zu}$ geringeren Preisen anbieten kann. Deshalb sollten StandAlone Kosten bei der Einfuhrung von Wettbewerb als ein hypothetisches Konzept betrachtet werden, das als Referenzpunkt furr wettbewerbsmäßige Preise bei Vorliegen von Größen- und Verbundvorteilen dienen kann. ${ }^{20}$ Dabei legen sie die Preisobergrenze fest $^{21}$, während Grenz- bzw. Inkrementalkosten die Preisuntergrenze darstellen. ${ }^{22}$

\subsubsection{Die Ramsey- PreisRegel}

Die hier zu analysierende Preisregel ist nach Frank Ramsey (1927), der sie auf optimale Besteuerung angewendet hat, und nach Marcel Boiteux genannt, der Ramseys Erkenntnisse zur Aufstellung von Regeln für einen Preisaufschlag auf Grenzkostenpreise für öffentliche Monopole ${ }^{23}$ verwendete. Baumol/Bradford (1970) entwickelten Boiteuxs Ansatz wiederum weiter. Diese Preisregel fokussiert einen Mehrgutermonopolisten, für den die Ermittlung von Endproduktpreisen anhand von Grenzkosten, inkrementellen Kosten oder Durchschnittskosten nicht praktikabel ist. Ursache hierfür ist, daß bei Herstellung mehrerer Produkte bzw. Dienste Gemeinkosten anfallen, die bei obigen genannten Konzepten nicht berlicksichtigt werden. Gemeinkosten lassen sich keiner bestimmten Bezugsgröße bzw. einzelnen Produkten (Kostenträgern) exakt zurechnen, weil sie von dem gesamten Unternehmen oder einer Organisationseinheit bei der Produktion mehrerer

20 Vgl. Albach/Knieps (1997), S. $19 \mathrm{f}$.

21 Stand-Alone Kosten dienen nur unter Ausschluß negativer Verbundvorteile als obere Preisgrenze. Zu negativen Verbundvorteilen vgl. Gerpott u.a. (1998), S. 1296.

22 SAK werden hinsichtlich eines SAK-Tests zugrunde gelegt, der der Analyse dient, ob Dienste quersubventioniert werden. Sind die Erträge der einzelnen Dienste höher als ihre SAK, liegt die Vermutung der Quersubventionierung sehr nahe. Vgl. dazu Mitchell/Vogelsang (1991), S. 119 sowie Gabel (1991), S. 79.

23 1956 in einem Aufsatz in französischer Sprache und 1971 in einem Artikel in englischer Sprache 
Güter verursacht werden. Sie werden nicht direkt in die Kostenträgerrechnung übernommen, sondern fließen in die Kostenstellenrechung ein, werden dort weiter verrechnet und schließlich im Rahmen der Kalkulation mit Hilfe von Gemeinkostenschlüssel auf die Produkte verteilt.

In der angelsächsischen Literatur werden im Hinblick auf den Produktionsprozeß Gemeinkosten in ,joint ' und ,common costs' unterschieden. ${ }^{24}$ Lassen sich die einzelnen Produktionsprozesse nicht nach selbständigen Prozessen der einzelnen Güter zerlegen, dann spricht man von, joint costs' (d.h. nicht trennbare Produktion mehrerer Güter). Hierbei handelt es sich um Gemeinkosten, die sich einer bestimmten abgegrenzten Gruppe von Produkten, jedoch nicht einem einzelnen Endprodukt zuordnen lassen. Lassen sich dagegen die Produktionsprozesse der einzelnen Güter sauber voneinander trennen, dann liegen ,common costs ${ }^{`}$ vor (d.h. gemeinsame Nutzung von Inputs, die sich jederzeit wieder rückgängig machen lassen kann, was allerdings eine Duplizierung an Kosten mit sich bringen würde). Diese umfassen Unternehmensgemeinkosten, also Kosten, die alle unterschiedlichen Produkte des gesamten Unternehmens betreffen. ${ }^{25}$ Diese Unterscheidung ist in bezug auf die Analyse der Kostenzurechnung von Bedeutung. Im Folgenden wird, sofern die Unterscheidung nicht wichtig ist, allgemein von Gemeinkosten die Rede sein.

Die Ramsey-Preisregel stellt im strengen Sinn ein Vollkostenkonzept dar, weil sowohl variable wie fixe Einzelkosten als auch Gemeinkosten auf die einzelnen Produkte zugerechnet werden. ${ }^{26}$ Ramsey- Preise können als ,second-best-Preise‘ im Verhältnis zu Grenzkostenpreisen bezeichnet werden, weil sie das Ziel verfolgen, die beste Preiskombination zu ermitteln, bei der die geringste Menge an Konsumentenrente relativ zu den Grenzkostenpreisen verlorengeht. ${ }^{27}$ Bei der RamseyPreisregel geht es folglich um die Maximierung der Konsumentenrenten unter der gegebenen Beschränkung, die Gesamtkosten zu decken. Unter der Annahme, daß der Monopolist keine Verluste machen darf, wird auf die Grenzkosten ein ,markup ' aufgeschlagen, der sich an der inversen Elastizitätsregel orientiert. Um wie viel der Preis höher als der Grenzkostenpreis ist, hängt von der Preiselastizität der Nachfrage ab.

\footnotetext{
24 Vgl. u.a. Cave u.a. (1990); S. 508 ff.; Rabe (1989), S. 150; Laffont/Tirole (1000) S. 194.

25 Zu Gemeinkosten vgl. auch Winzer (2000), S. 103.

26 Vgl. Mitchell/Vogelsang (1991), S. 140; Rabe (1989), S. 151 ff.; Winzer (2000), S. 77. Klassische Preisfindungskonzepte auf der Grundlage von Vollkosten sind unter anderem ,Fully Distributed Costs' sowie der Prozeßkostenansatz. Vgl. hierzu Kap. 5.4.4.1.

Vgl. Baumol/Bradford (1970).
} 
Ramsey-Boiteux-Preise unterstellen die Existenz eines benevolenten Regulators, der über vollkommene Information verfügt und zum Wohle aller handelt. ${ }^{28} \mathrm{Die}$ ses Konstrukt ist entsprechend seiner hehren Annahmen vollkommen irreal. Es wird hier aber als Analysegegenstand verwendet, weil es ein allokativ effizientes Ergebnis formuliert.

Für die Herleitung ${ }^{29}$ der allgemeinen Ramsey-Preise werden folgende zentrale Annahmen unterstellt: Das Mehrproduktunternehmen stellt die Güter $i=1,2, \ldots, n$ her. Dabei entspricht $x_{t}$ dem produzierten Output von Gut $i$. Die totalen Kosten des Unternehmens fur alle produzierten Güter bestehen aus zwei Kostenkomponenten, den Gemeinkosten $k$ und den outputabhängigen Kosten für alle hergestellten Güter:

$$
K(x)=k+\sum_{i=1}^{n} c_{i} x_{i}
$$

Die Kostenkurve verläuft linear. Daraus ergeben sich konstante Grenzkosten, die hier als $c_{i}$ bezeichnet werden.

Die Ertragsfunktion des Mehrproduktunternehmens lautet:

$$
E(x)=\sum_{i=1}^{n} p_{i}\left(x_{i}\right) x_{i},
$$

wobei $p_{i}$ dem Endproduktpreis des jeweiligen hergestellten Gutes entspricht.

Die Nachfrage nach Gut $i$ wird als $x_{i}=x_{i}^{\nu}\left(p_{i}\right)$ bezeichnet. Es wird unterstellt, daß die Nachfrage nach den Gütern $i=1,2, \ldots, n$ hier der Einfachheit halber voneinander unabhängig ist. Es ergibt sich folgende Brutto-Konsumentenrente $S(x)$

$$
S(x)=\int_{0}^{n} p_{i}\left(x_{i}\right) d x_{i},
$$

wobei die nachgefragte Menge nach Gut $i$ bei 0 Einheiten beginnt und bei $n$ Einheiten endet. Es gilt:

$$
\frac{\partial S}{\partial x_{i}}=p_{i}
$$

28 Dieses Konzept ist grundsătzlich angreifbar, da die Annahme über die Existenz eines benevolenten Regulators höchst fragwilrdig ist. Vgl. dazu Kap. 6. Es soll hier allerdings zunächst nicht weiter hinterfragt werden.

29 In Anlehnung an Braeutigam (1998), S. 1320 ff.; Train (1991); S. 125 ff.; Laffont/Tirole (2000), S. 64. 
Das verfolgte Ziel, nämlich eine optimale Preisstruktur für das Unternehmen zu finden, so daß es den ,break-even point' erreicht bzw. nicht-negative Gewinne erwirtschaftet, wird hier einem wohlwollenden Regulator in die Hände gelegt, der über vollkommene Information über Kosten und Nachfrage verfügt und dem keine Anreizprobleme gegenüberstehen.

Die soziale Wohlfahrt läßt sich wie folgt definieren: $W=S(x)-K(x) \rightarrow \max x$. Die Maximierung soll unter der gegebenen Restriktion, daß das Unternehmen keine Verluste macht: $E(x)-K(x)=0$, erfolgen. ${ }^{30}$

Für die Güter bzw. für Dienste $i=1, . . n$ lassen sich Ramsey-Boiteux-Preise wie folgt unter der Annahme herleiten, daß die Nachfragen nach den einzelnen Gütern voneinander unabhängig sind:

$$
F=S\left(x_{i}\right)-K\left(x_{i}\right)+\lambda\left[E\left(x_{i}\right)-K\left(x_{i}\right)\right] .
$$

Nach Maximierung von $F$ erhält man

$$
p_{i}-c_{i}+\lambda\left(\frac{\delta p_{i}}{\delta x_{i}} x_{i}+p_{i}-c_{i}\right)=0 .
$$

Mit Hilfe einer Erweiterung im Klammerterm läßt sich die inverse Nachfrageelastizität $1 / \eta$ einfügen: ${ }^{31}$

$$
\begin{aligned}
& p_{i}-c_{i}+\lambda\left[p_{i}-\left(\frac{p_{i}}{\eta_{x_{i}, p_{i}}}\right)-c_{i}\right]=0 \\
& p_{i}-c_{i}=-\lambda\left[-\left(\frac{p_{i}}{\eta_{x_{i}, p_{i}}}\right)+p_{i}-c_{i}\right]
\end{aligned}
$$

$$
p_{i}-c_{i}+\lambda\left(p_{i}-c_{i}\right)=\lambda \frac{p_{i}}{\eta_{x_{i}, p_{i}}}
$$

$$
\frac{p_{i}-c_{i}}{p_{i}}+\lambda \frac{p_{i}-c_{i}}{p_{i}}=\lambda \frac{1}{\eta_{x_{i}, p_{i}}}
$$

\footnotetext{
30 Alternativ läßt sich ebenso das wohlfahrtsoptimale Ergebnis bestimmen, in dem die Gewinnfunktion des Unternehmens maximiert wird unter der gegebenen Restriktion $S(x)$ $\mathrm{C}(\mathrm{x}) \geq \mathrm{S}\left(\mathrm{x}^{*}\right)-\mathrm{C}\left(\mathrm{x}^{*}\right) ; \mathrm{x}^{*}$ steht für das Ramsey-Boiteux-Niveau.
}

31 Es gilt im folgenden : $\partial C / \partial x_{i}=c_{i}$. 


$$
\begin{aligned}
& (1+\lambda) \frac{p_{i}-c_{i}}{p_{i}}=\lambda \frac{1}{\eta_{x_{i}, p_{i}}} \\
& \frac{p_{i}-c_{i}}{p_{i}}=\frac{\lambda}{1+\lambda} \frac{1}{\eta_{x_{1}, p_{i}}} \cdot{ }^{32}
\end{aligned}
$$

$p_{i}-c_{i}$ repräsentiert das Ausmaß der Abweichung der Preise von den Grenzkosten und stellt folglich den Preisaufschlag auf den Grenzkostenpreis dar. Der Ausdruck wird durch $p_{i}$ dividiert, um ihn als Preisverhältnis auszudrücken. ${ }^{33}$

$\lambda$ ist ein Schattenpreis fur die Budgetrestriktion des Incumbents. Der Term $\lambda / 1+\lambda$ stellt dementsprechend die Abweichung von den Grenzkosten dar. Es gilt: $0 \leq \lambda / 1+\lambda \leq 1$. Ist die Nebenbedingung nicht bindend, dann ist $\lambda=0$ und dementsprechend $\lambda / 1+\lambda=0$. Dann würde der gewăhlte Preis den Grenzkosten entsprechen. Gilt aber $\lambda>0$, dann ist dementsprechend $\lambda / 1+\lambda>0$. Daraus folgt, daß die break-even- Bedingung bindend fur das Erreichen eines Optimums und höher als die Grenzkostenpreise ist.

Die Berücksichtigung der inversen Preiselastizität der Nachfrage in (5.1.12) soll dazu beitragen, einen Preis möglichst ohne einen Verlust für das Unternehmen zu formulieren. Unter der Annahme konstanter Grenzkosten und auf den hier relevanten Produktmärkten voneinander unabhängigen Nachfragefunktionen ergibt sich entsprechend der inversen Elastizitätsregel : je weniger elastisch die Nachfrage nach den einzelnen Produkten reagiert, desto höher kann der Preisaufschlag auf die Grenzkosten sein. ${ }^{34}$

Ramsey-Preise führen nicht zwangsläufig zu der Etablierung der monopolistischen Stellung des Anbieters. Der Preisaufschlag kann beispielsweise aufgrund vollkommen unelastischer Nachfrage so hoch sein, daß es sich für konkurrierende Anbieter lohnen würde, bestimmte Produkte zu duplizieren oder Substitute zu entwickeln. Bei der Ramsey-Preisregel handelt es sich um eine Mehrproduktregulierung, die alle Produkte bzw. Dienste $i=1, \ldots, n$ im Endproduktmarkt betrifft. Sie ist ursprünglich nicht für die Existenz eines vor- und eines nachgelagerten Marktes konzipiert, kann aber dahingehend uminterpretiert werden, wie es in Kapitel 5.2.2 erfolgt.

Im Zusammenhang mit der Anwendung der Ramsey-Regel soll auf folgende gravierende Probleme hingewiesen werden, wenn mit ihr regulierend auf die Märkte Einfluß genommen werden soll: Damit regulierende Eingriffe auf Grundlage der

32 Hier werden die Ramsey-Preise nicht ausfuhrlicher hergeleitet, sondern nur insoweit, wie sie für die spezielle Herleitung für Zugangsentgelte in Kap. 5.2.2 vonnöten ist.

33 Vgl. Train (1991), S. 124.

34 Detaillierte Analyse bezüglich der Berücksichtigung von Kreuzpreiselastizitäten und die Aufhebung der Annahme einer linearen Nachfrage vgl. hierzu Train (1991), S. 139 ff. 
Ramsey-Regel zu allokativer und produktiver Effizienz führen, muß ein alleswissender wohlwollender Regulator unterstellt werden, der zum Ziel hat, die soziale Wohlfahrtsfunktion zu maximieren. Gemäß der Erkenntnisse aus der Public Choice- Theorie kann von der Existenz eines solchen Regulators in der Praxis nicht ausgegangen werden. Anstelle seines wohlwollenden Handelns muß die Prämisse ausschließlicher Eigennutzorientiertheit gemacht werden. ${ }^{35}$ Um Grenzkosten und Nachfragefunktionen bzw. Elastizitäten zu messen, erfordert insbesondere letztere einen sehr hohen Informationsbedarf. Es muß davon ausgegangen werden, daß einem Regulator niemals alle notwendigen Informationen zur Verfügung stehen werden, weil die Betroffenen in der Regel nicht einmal selbst darüber verfügen. Davon abgesehen verursacht jede Beschaffung von Informationen Kosten, die hier nicht berücksichtigt wurden. Die Besorgung der hier notwendigen Information kann unwirtschaftlich bzw. sogar unmöglich sein. Man könnte sich behelfen, indem Schätzungen vorgenommen werden. Das hieße, daß die RamseyPreisregel mit unzureichenden Informationen durchgeführt werden müsste. Daraus folgt, daß die Ermittlung des Preisaufschlages auf Basis der Ramsey-Preise nicht exakt errechnet werden $\mathrm{kann}^{36}$ und dies sich verzerrend auf den Markt auswirkt.

Dies mögen Argumente dafür sein, daß diese Preisregel in der Empirie bisher noch nicht angewendet worden ist. Die Ramsey-Regel darf eben nicht als politische Handlungsempfehlung mißverstanden werden, weil ihre Durchführung nicht exakt möglich ist. Sie kann aber dazu dienen, ein theoretisch sauberes allokativ effizientes Ergebnis zu ermitteln. Eine Anwendung der Ramsey-Preisregel auf den Telekommunikationsmarkt wird in Kap. 5.2.2 vollzogen.

\subsubsection{Die ,Efficient Component Pricing Rule'}

Die Efficient-Component-Pricing-Rule (ECPR), auch bekannt als Paritäts-PreisPrinzip, kompetitive Neutralitätsformel oder als Baumol-Willig-Regel, wurde erstmals von Willig (1979) vorgeschlagen. Baumol (1983) interpretierte die formalen Ergebnisse von Willig und propagierte sie später in bezug auf den Telekommunikationsmarkt. ${ }^{37}$ Die ECPR wird im Rahmen einer Einzelpreisregulierung für ein Mehrproduktunternehmen, welches auf einem vor- und einem nachgelagerten Markt agiert, angewendet. Dabei ermittelt sie ein Preisgleichgewicht für

\footnotetext{
35 Vgl. hierzu Kap. 6, insbesondere Kap. 6.2.1.

36 Vgl. Ambak u.a. (1994), S. 10.

37 Vgl. dazu Baumol/Sidak (1994b) und Kap. 5.2.3.
} 
den Zugang zu einem vorgelagerten Markt, das sich aus der Verhandlung der betroffenen Parteien ergibt.

Es wird von der Existenz zweier Märkte ausgegangen. Auf dem einen Markt wird ein Produkt vertrieben, welches einen wesentlichen Input fur den anderen Markt darstellt. Wegen dieser einseitigen Abhängigkeit lassen sich diese Mărkte gut als vor- und als nachgelagerter Markt bezeichnen. Außerdem wird davon ausgegangen, daß es auf dem Input-Markt nur einen einzigen Anbieter gibt. Aus diesem Grund kann der vorgelagerte Markt auch als Bottleneck bezeichnet werden. ${ }^{38}$ Weiterhin wird hier unterstellt, daß der Incumbent sowohl auf dem vorgelagerten als auch auf dem nachgelagerten Markt agiert. Die Newcomer dagegen treten nur auf dem nachgelagerten Markt ein. Für den Markteintritt benötigen sie Inputs aus dem vorgelagerten Markt. Die zentrale Frage lautet nun: Welchen Preis kann der Incumbent von einem Entrant fur den Kauf seines Inputs nehmen? Dabei geht es nicht nur um die Deckung der anfallenden Kosten furr die Bereitstellung der Inputs, die von den Entrants genutzt werden. Der Gewinnentgang auf dem nachgelagerten Markt, den der Incumbent erleidet, dadurch daß er dem Entrant einen Markteintritt ermöglicht, muß ebenso kompensiert werden. Dieser Gewinnentgang wird auch als Opportunitätskosten bezeichnet. Ein Input-Preis soll deswegen neben den angefallenen Kosten ebenso die Opportunitätskosten beinhalten:

\section{Optimaler Input-Preis $\left(p_{\text {Input }}\right)=$ direkt entstandene Kosten für Inputs pro Einheit + Opportunitätskosten, die der Input-Anbieter pro Verkauf einer Einheit an Input hat ${ }^{39}$.}

Baumol/Willig interpretieren die direkt entstandenen Kosten als durchschnittliche inkrementelle Kosten im Rahmen der Theorie bestreitbarer Märkte. ${ }^{40}$

Der Inputhersteller veräußert Inputs an Wettbewerber im nachgelagerten Markt. Die Wettbewerber können dadurch Endprodukte bereitstellen, die sonst der Inputhersteller alleine hätte verkaufen können. Deswegen stellen die Opportunitätskosten entgangene potentielle Nettoerträge dar. Folglich ergibt sich der Inputpreis aus der Differenz zwischen dem Endproduktpreis, zu dem der Incumbent das Endprodukt bereitstellt - $p_{\text {End, }}$ - und den für den Incumbent angefallenen relevanten Kosten ${ }^{41}$ :

$$
p_{\text {Input, }}=p_{\text {End, }}-\text { relevante Kosten } \text { Input, }_{\text {. }} \text {. }
$$

\footnotetext{
38 Beispiele hierfür sind die Netzinfrastruktur bei Netzökonomien wie der Telekommunikation oder auch des Schienenverkehrs.

39 Vgl. Baumol/Sidak (1994b) S. 94 f.

40 Vgl. dazu Baumol u.a. (1988), S. 67 sowie Kap. 5.1.3.

41 Vgl. Baumol (1999), S. 576.
} 
Unter relevanten Kosten sind die Kosten zu verstehen, die nicht entstanden wären, wenn dem Rivalen kein Zugang zum Input gewährt worden wäre und der Incumbent stattdessen den Output im nachgelagerten Markt abgesetzt hätte. Sie umfassen neben den tatsächlich anfallenden Kosten ebenso die Opportunitätskosten.

Die Anwendung der ECPR führt dazu, daß potentielle Wettbewerber nur dann in den nachgelagerten Markt eintreten, wenn sie ihre Endprodukte technisch bzw. produktiv effizienter anbieten als der Incumbent. ${ }^{42}$ Außerdem ist die Regel ertragsneutral für den Incumbent und läßt die Eigentumsrechte des Incumbents unberührt. Mit ihrer Anwendung eliminiert sie Anreize des Incumbents, Rivalen aus dem Markt zu drängen. Weiterhin macht es ihre Anwendung so attraktiv, weil ihre regulatorischen Anforderungen minimal sind und sie darauf ausgerichtet ist, daß sich die betroffenen Parteien in bezug auf die Höhe des Zugangsentgelts untereinander einigen ohne Einschaltung einer dritten Instanz.

Im Vergleich zu den Ramsey-Boiteux-Preisen wird die Nutzerseite nicht berücksichtigt. Das hat den Vorteil, daß der Informationsaufwand geringer ist, da z.B. keine Schätzung der Nachfragefunktion notwendig ist. Die ECPR konzentriert sich mehr auf das Ziel produktiver Effizienz ${ }^{43}$ im Gegensatz zu der RamseyPreisregel, bei der die allokative Effizienz im Vordergrund steht.

Ob die ECPR ebenso zu allokativer Effizienz führt, hängt von gewissen Annahmen über den nachgelagerten Markt ab. Die entscheidende Annahme für die allokative Effizienz der Regel ist die Unterstellung, daß der Incumbent auf dem nachgelagerten Markt für Endprodukte zu einem Preis, der den Grenzkosten entspricht,

42 Vgl. u.a. Baumol/Sidak (1994b), S. 107 und (1994a), S. 181 f.; Economides/White (1995), S. 559.

43 Economides/White bezweifeln die Bedeutsamkeit produktiver Effizienz. Vgl. Economides, White (1995), S. 565 ff. Sie wollen in ihrer Kritik deutlich machen, daß es vom wohlfahrtsökonomischen Standpunkt her zu einem gesamtgesellschaftlichen Nettogewinn kommen kann, wenn ineffiziente Anbieter auf den Markt eintreten. Dem Gewinn an Konsumentenrente und Produzentenrente steht ein Verlust durch einen technisch ineffizienten Markteintritt gegenüber. Ob der Nettogewinn letztendlich positiv ist, hängt neben dem Verlauf der Nachfrageelastizität zusätzlich von der Höhe der zusätzlichen Kosten ab, die beim Marktantritt des Entrants anfallen (da angenommen worden ist, da $B$ der Entrant ineffizienter produziert, hat er dementsprechend höhere Kosten als der Incumbent). Außerdem hängt das Ergebnis von der Höhe des Marktanteils des Entrants ab. Es wird eine ,limit pricing' Strategie des Monopolisten unterstellt. Sie besagt, daß der Preis von dem Monopolisten so gesetzt wird, da $B$ beide Produzenten auf einem Niveau verkaufen, das den Kosten des Entrants entspricht. Hier wird angenommen, daß dieser Preis über den Grenzkosten, aber unter dem Monopolpreis liegt. Das Ergebnis ist, daß es sowohl bei Bertrand- als auch bei Cournot- Wettbewerb unter der Annahme bestimmter Elastizitäten und Marktanteile zu positiven Nettogewinnen kommt. Economides und Whites Schlußfolgerung lautet: Je höher die Anzahl der Wettbewerber, desto höher der Nettowohlfahrtsgewinn. Ziel dieser Argumentation ist es, die Bedeutung der produktiven Effizienz zu entkräften. Die angewendete Methodik ist allerdings ebenso bestreitbar wie das Ergebnis. 
an die Endkunden verkauft. ${ }^{44}$ Dies ist eine äußerst restriktive Annahme. Sie unterstellt, daß schon perfekter Wettbewerb auf dem nachgelagerten Markt besteht.

Fur Branchen, in denen der Markteintritt alternativer Anbieter erst erfolgt, ist anzunehmen, daß der Incumbent bisher als Monopolist auf beiden Märkten agiert hat. Dann gäbe es für ihn keine Anreize, einen Grenzkostenpreis für das Endprodukt zu nehmen. Stattdessen wird er eine Monopolrente auf den Endproduktpreis aufschlagen, dessen Höhe sich durch die Preiselastizität der Nachfrage bestimmt. Baumol/Sidak wiesen selbst auf dieses Problem hin. Dabei betonen sie, daß die ECP-Regel damals ${ }^{45}$ unter der Voraussetzung regulierter Endproduktpreise entworfen wurde, die das Bestehen von Grenzkostenpreisen für das Endprodukt im nachgelagerten Markt gewährleisteten bzw. daß dort keine Monopolpreise vorherrschen. Der Endproduktpreis, den der Monopolist setzt, sollte nicht den Preis, der sich bei Markteintritt eines effizienten Anbieters ergeben würde, sprich gemäß den Stand-Alone-Kosten (SAK), ubersteigen. ${ }^{46}$

Es läßt sich folglich festhalten: Ob die Regel allokativ effizient ist, hängt davon ab, ob der Endproduktpreis den Stand-Alone Kosten entspricht. Wenn der SAKPreis sich nicht durch einen Preiswettbewerb zwischen Anbietern ergibt, muß eine Regel für die Bepreisung von Endprodukten regulatorisch festgelegt werden, damit die ECPR ein allokativ effizientes Ergebnis erzielt. ${ }^{47}$ Ansonsten kann die ECPR mißbraucht werden, Monopolprofite zu konservieren und zu einer ineffizienten Ressourcenallokation zu führen. ${ }^{48}$

Weitere implizit unterstellte Annahmen hinsichtlich des Erreichens eines produktiv und allokativ effizienten Ergebnisses sind:

- Die SAK aus der Produktion seitens des Monopolisten sind für den regulatorischen Eingriff im nachgelagerten Markt akkurat ermittelbar.

- Der nachgelagerte Markt ist homogen, d.h. Endprodukte sind perfekte Substitute. Nur dann entspricht der Nachfragerückgang in voller Höhe dem Gewinnentgang des Incumbents. ${ }^{49}$

- Entrants sind Preisnehmer im Dienstemarkt, d.h. haben keine Marktmacht.

\footnotetext{
Vgl. Tye (1994) S. 206 f., Economides/White (1995), S. 560.

Vgl. Baumol (1983).

Vgl. Baumol/Sidak (1994b), S.108; Baumol/Sidak (1994a), S. 195.

Vgl. Tye (1994), S. 210.

48 Vgl. Economides/White (1995), die den Fall von Monopolpreisen im nachgelagerten Markt explizit analysieren.

49 Vgl. Armstrong/Doyle (1995), S. 11. Sind die Güter beider Anbieter auf dem nachgelagerten Markt gar nicht substituierbar, dann sollte das Zugangsentgelt nach Armstrong und Doyle lediglich die für die Netzzugangsge-währung unmittelbar anfallenden Kosten beinhalten. Bei nicht perfekten Substituten ist die Einfuhrung einer Variable, die den Verdrängunggsgrad aufgrund von Substituierbarkeit gewichten soll. Vgl. dazu Kap. 6.2.4; Armstrong/Doyle/Vickers (1996), S. 138 ff.; Armstrong/Doyle (1995), S. 11; Valletti/Estache (1999), S. 20 f.
} 
- Entrants haben keine Fixkosten und ebenso keine versunkenen Kosten.

- Die Produktionstechnologie erfolgt unter konstanten Skalenerträgen in bezug auf den nachgelagerten Markt.

- Die Nachfragefunktionen des Incumbents und des Entrants sind symmetrisch. D.h. es gibt keine Markenloyalität und keine Wechselkosten.

- Die Kostenfunktionen im kompetitiven nachgelagerten Markt sind symmetrisch. Die SAK für die Dienstebereitstellung sind für den Incumbent wie für den Newcomer gleich.

Trotz dieses restriktiven Annahmegerüsts fand die ECP-Regel im Gegensatz zu der Ramsey-Regel, dessen Annahmen ungleich weniger restriktiv sind, in der Realität tatsächlich Anwendung, z. B. bei der Eisenbahnregulierung in den USA ${ }^{50}$. Außerdem wurde sie als Interconnection-Regelung der British Telecom in Großbritannien und der Telecom New Zealand in Neuseeland vorgeschlagen ${ }^{51}$.

Die Stärke der ECPR liegt darin, produktive Effizienz auf dem Endproduktmarkt zu erreichen, indem bei einem Markteintritt um effiziente Rivalen miteinander im Wettbewerb zueinander stehen. So kann die ECPR einen dauerhaft wirksamen Wettbewerb gewährleisten. Soll das Ziel der ECPR die Ermittlung eines Zugangsentgelts sein, das zur allokativen Effizienz führt, dann müssen Grenzkostenpreise auf dem nachgelagerten Markt gewährleistet sein.

\subsection{MöGLICHKEITEN DER FESTLEGUNG EINES ZugANGS- ENTGELTS BEI EINEM ONE-WAY-ACCESS}

Nachdem einige Preisregeln in Kapitel 5.1 kurz vorgestellt worden sind, sollen sie nun auf die Regelung des Zugangs zum vorgelagerten Markt angewendet werden. Dabei sollen sie hinsichtlich ihrer Fähigkeiten verglichen werden, inwieweit es ihnen gelingt, Wettbewerb auf dem nachgelagerten Markt einzuführen.

so Vgl. Baumol (1983). Fälle bekannt als Compensation I through IV, St. Louis S.W. Ry.Trackage Rights over Missouri Pac. R.R. - Kansas City to St. Louis, 1 I.C.C. 2d 776 (1984), 4 I.C.C. 2d 668 (1987), 5 I.C.C. 2d (1989), 8 I.C:C. 2d 80 (1991). Vgl. Baumol/Sidak (1994a), S. 180.

51 Vgl. dazu Kap. 5.2.3. Clear Communications, Ltd. V. Telecom Corp. Of New Zealand, CP590/91, slip op. (H.C. Dec. 22, 1992) (available at the Yale Law Library), rev'd, C.A. 2593, slip op. (C.A. Dec. 17, 1993) (available at the Yale Law Library). Vgl. Baumol/Sidak (1994a), S. 180. 


\subsubsection{DER ONE-WAY-ACCESS}

Bei Markteintritt eines Anbieters auf einen nachgelagerten Markt ist für die effiziente Bereitstellung eines Dienstes der Zugang zu den vorgelagerten Märkten den Netzzugang betreffend - erforderlich. Der Netzzugang umfaßt neben den Zugang auf die Infrastrukturebene, also den Teilnehmeranschlußleitungen, den Übertragungswegen und den Vermittlungseinrichtungen, ebenso Vorleistungen wie die Möglichkeit der Miete von Teilnehmeranschlußleitungen sowie von Mietleitungen und der Zusammenschaltung von Netzen verschiedener Betreiber. ${ }^{52}$ Für den Netzzugang gelten zwei Attribute: ${ }^{53}$ Erstens ist der Zugang ein Input, der für das Angebot des Endproduktes unbedingt notwendig ist. Zweitens produziert der Anbieter des Zugangs diesen Input zum einen für sich selbst, zum anderen für wettbewerbliche Anbieter auf nachgelagerten Märkten für Endprodukte. Dieser sogenannte ,One-Way-Access' betrifft ausschließlich Wettbewerber, die über keine eigene Infrastruktur im Teilnehmeranschlußbereich verfügen, also sowohl reine Wiederverkäufer von Telekommunikationsdiensten als auch DiensteAnbieter, die in der Regel nur eine geringe Anzahl an Switches im Weit- und/oder Nahverkehrsbereich besitzen, nicht aber auf der Letzten Meile, sowie substitutive Anbieter, deren Netz sich im Aufbau befinden.

\subsubsection{DER WIEDERVERKÄUFERWETTBEWERB}

Bei der Bestimmung des Zugangsentgelts bei reinem Wiederverkăuferwettbewerb ist der Ausgangspunkt der Betrachtung ein vertikal integriertes dominantes Unternehmen. Der Incumbent entspricht einem Mehrproduktunternehmen, nämlich einem Netzbetreiber, der ebenso Endkundendienste anbietet oder im Rahmen von Vorleistungen vermietet.

In Abbildung 5.2-1 wird neben dem Verbindungsaufbau eines Telekommunikationsdienstes, wie zum Beispiel eines Telefonates, uber die physische Netzinfrastruktur in Form einer durchgezogenen Linie der logische Verbindungsaufbau, der die anfallenden Kosten furr einen einfachen Telefondienst widerspiegelt, in Form einer gestrichelten Linie dargestellt. Der physische Verbindungsaufbau beginnt bei einem Teilnehmeranschluß, läuft über verschiedene Vermittlungsstellen, die hier in Form von Kreisen und Vierecken ${ }^{54}$ abgebildet sind, und endet bei dem gewünschten Teilnehmeranschluß. Der logische Verbindungsaufbau kennzeichnet

\footnotetext{
52 Vgl. dazu Abbildung 2.1.-3 in Kap. 2.1.2 sowie Kap. 2.4.2.1.

53 Vgl. Baumol/Sidak (1994a), S. 172 f.

54 Die Kreise stehen fur Vermittlungsstellen auf der Nahbereichsebene, die Vierecke für Vermittlungsstellen auf der Weitverkehrsebene.
} 
die Originierung, die Transitleistung und die Terminierung eines Dienstes. ${ }^{55}$ Die dabei anfallenden Grenzkosten entsprechen $2 c_{0}+C$. $c_{0}$ steht für Grenzkosten, die bei Verbindungsaufbau im Teilnehmeranschlußbereich für die Originierung und Terminierung anfallen: Gemeint ist damit die Strecke zwischen Teilnehmeranschluß bis zur ersten VSt und die Strecke von der letzten VSt bis hin zum Empfänger des Telefongesprächs. $C$ ist der Teil der Grenzkosten, der sich durch die Transitleistung des Dienstes ergibt. ${ }^{56}$

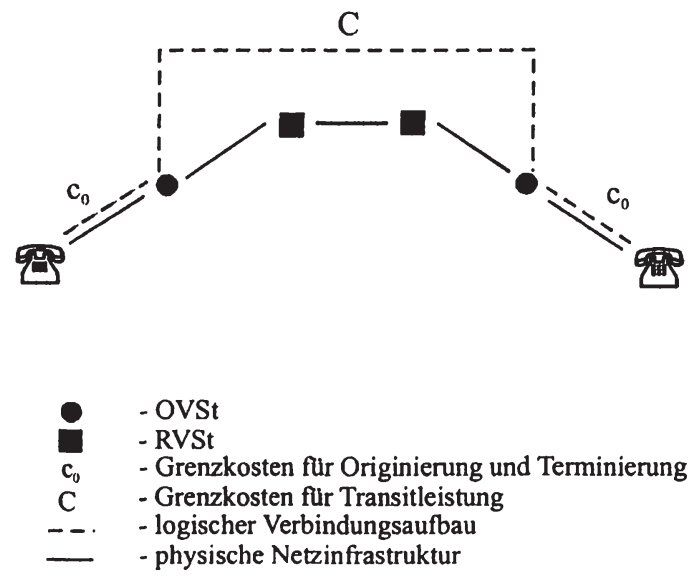

\section{Abbildung 5.2-1: Wiederverkäuferwettbewerb}

Ein Wiederverkäufer mietet neben der Originierung und der Terminierung auch die Transitleistungen von dem Incumbent an.

Die Kostenfunktion des Incumbents $I$, der hier zunächst alleiniger Produzent auf dem vor- und nachgelagerten Markt ist, lautet: $K_{I}=K^{\text {Nerze }}+K^{\text {Diensle }}$.

$$
K_{I}=k_{I}+c_{0}^{2} X+C X,
$$

wobei $k$, den Einzelfixkosten sowie den Gemeinkosten, die für den Incumbent anfallen, entspricht und $X$ der gesamten Nachfrage auf dem Endproduktmarkt. ${ }^{57}$

35 Vgl. dazu vertikale Marktabgrenzung in Kap. 3.3.3.1.

s6 In der Realität liegt in diesem Kostensegment Distanzkostendegression vor. Sie besagt, je größer die geographische Entfernung zwischen den beiden Gesprächspartnern ist, desto billiger wird die Distanzüberwindung. Vgl. dazu v. Weizsäcker (1997), S. 573. Hier wird dieser Gesichtspunkt zunächst vernachlässigt und von linearen Kosten ausgegangen.

57 Die quadratische Spezifikation der Originierungs- und Terminierungskosten vermeidet technische Schwierigkeiten bei der Grenzkostenbetrachtung. 
Bei Wiederverkäuferwettbewerb wird angenommen, daß der Incumbent im nachgelagerten Markt die Gesamtmenge an Diensten herstellt, wobei er einen Teil der Dienste selbst verkauft und den anderen Teil einem Entrant bereitstellt, der diese wiederum an den Endkunden weiterveräußert. Der Newcomer tritt ausschließlich als Wiederverkäufer auf dem nachgelagerten Markt auf. Hier sei zunächst unterstellt, daß die Nachfrage nach Diensten seitens der Konsumenten voneinander unabhängig ist. Dabei gilt: $X=x_{1}+x_{2}$, wobei $x_{1}$ der Menge an von Konsumenten nachgefragten Diensten entspricht, die vom Incumbent bereitgestellt werden und $x_{2}$ für die Menge an Diensten steht, die der Incumbent an den Entrant vermietet, der sie wiederum an Endkunden weiterverăußert. Die Notation 1,2 kennzeichnet hier also den Anbieter $(B=1,2)$. Grundsätzlich müßten die bereitgestellten Mengen mit $x_{i_{1}}, x_{i_{2}}$ abgekürzt werden. Da aber der Einfachheit halber davon ausgegangen wird, daß beide Anbieter nur eine Diensteart anbieten, die sich nicht hinsichtlich ihrer Qualität, Erreichbarkeit oder Vielfaltigkeit unterscheiden, kann hier synonym von $x_{1}, x_{2}$ gesprochen werden. ${ }^{58}$

Dementsprechend teilen sich die Grenzkosten für die Transitleistung auf in $C=c_{1}+c_{2}$. Die Kostenfunktion des Incumbents kann dann umformuliert werden:

$$
K_{I}=k_{1}+c_{0}^{2}\left(x_{1}+x_{2}\right)+c_{1} x_{1}+c_{2} x_{2}
$$

$$
\frac{\partial K_{1}}{\partial x_{1}}=2 c_{0}+c_{1}
$$

$$
\frac{\partial K_{1}}{\partial x_{2}}=2 c_{0}+c_{2}
$$

$2 c_{0}+c_{1}$ entsprechen den Grenzkosten für Dienste, die der Incumbent selbst vertreibt. $2 c_{0}+c_{2}$ sind die Grenzkosten, die dem Incumbent für die Herstellung von Diensten anfallen, die er an den Entrant vermietet, die dieser wiederum weiterveräußert. 59

Der Newcomer veräußert $x_{2}$ Einheiten an den Endkunden zu einem Preis von $p_{2}$ und erwirtschaftet dementsprechend den Erlös $p_{2}\left(x_{2}\right) x_{2}$. Der niedrigste Preis,

58 Das Prinzip des Modells läßt sich auf alle Sprachbasisdienste sowie sämtliche sprach-, datenoder videogestützte Breitbanddienste übertragen.

59 Das hier vorzustellende Modell ist an Laffont/Tirole (2000), S. 100 f. und Laffont/Tirole (1996), S. 232 ff. angelehnt. Laffont/Tirole grenzen die zu analysierenden Märkte in Ortsnetz und Fernnetz ab, wobei das Ortsnetz als vorgelagerter Markt mit Bottleneck bzw. Essential Facilities - Eigenschaften behaftet ist. Da hier eine Abgrenzung Netzinfrastruktur und Vorleistungen - versus Endkundendienste gemacht worden ist, unterscheidet sich das Modell von Laffont/Tirole dahingehend. 
den der Neuanbieter für seine Dienste nehmen kann, ergibt sich, wenn die Grenzerlöse aus dem Wiederverkauf den anfallenden Grenzkosten entsprechen:

$$
p_{2}=2 c_{0}+c_{2} .
$$

An diesem Punkt macht der Newcomer nach Markteintritt keine Gewinne. Er ist an diesem Punkt indifferent, ob er in den Markt eintritt oder nicht.

Für den Wiederverkäufer ist die Gewährung des Zugangs zu den vorgelagerten Märkten nicht wirklich ein Problem, da er ohnehin alles anmietet, d.h. Kapazitäten auf dem nachgelagerten Markt für den bereitgestellten Telefondienst wie die dazu notwendigen Kapazitäten auf den vorgelagerten Märkten. Vermutlich wird er dies als Gesamtpaket dem Incumbent abkaufen und durch Mengenrabatte günstige Konditionen für Endkunden anbieten können und dabei selbst noch einen positiven Gewinn machen.

\subsubsection{DER DIENSTEWETTBEWERB}

Der Dienstewettbewerb unterscheidet sich von dem reinen Wiederverkäuferwettbewerb insofern als der Entrant eine eigene geringfuigige Infrastruktur auf der Transitebene in Form von einer geringen Zahl an Switches besitzt, wie an der Abbildung 5.2-2 deutlich zu sehen ist. ${ }^{60}$ Die Netzstruktur des Entrants wird in der Abbildung in Form von Dreiecken und der Verbindungslinie zwischen den beiden Dreiecken dargestellt. Der Newcomer ist auf den Zugang zu dem Netz des Incumbents angewiesen, der in der Abbildung nach wie vor den Teilnehmeranschlußbereich monopolisiert. Der Entrant benötigt einen Zugang zu einer Vermittlungsstelle auf der Ortsnetzebene, um eigene Dienste $x_{2}$ vermitteln zu können. Der Zugang zu der Infrastruktur des Incumbents ist in der Abbildung dadurch gekennzeichnet, daß die Netzstruktur des Newcomers an eine bzw. hier an zwei VSt des Incumbents auf der Ortsebene anknüpft.

Die anfallenden Kosten bei Inanspruchnahme eines Dienstes lauten für den Incumbent:

$$
G K_{I}=2 c_{0}+c_{1} .
$$

Der Entrant hingegen muß auf seine Grenzkosten für die Transitleistung auf der eigenen Netzinfrastruktur noch das Zugangsentgelt $a$ rechnen:

$$
G K_{E}=c_{2}+a \text {. }
$$

Er muß sowohl für die Originierung als auch für die Terminierung eines zu vermittelnden Gesprächs den Teilnehmeranschlußbereich des Incumbents zu einem

60 Hinsichtlich des Dienstewettbewerbs vgl. Kap. 3.1.2. 
Preis von $a$ nutzen können. Die Höhe des Zugangspreises ist für weitere Marktentwicklungen von besonderer Bedeutung.

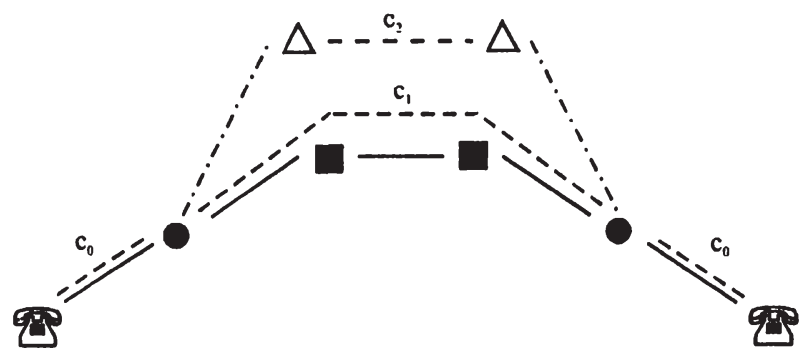

- - OVSt des Incumbents

- WVSt des Incumbents

$\triangle \quad$ - WVSt des Entrants

$c_{n} \quad$ - Grenzkosten für Originierung und Terminierung

$c_{1}, c_{2}$ - Grenzkosten fur Transitleistung

-.. - logischer Verbindungsaufbau

- - physische Netzinfrastruktur

-. - . Zugang zum Netz des Incumbents

\section{Abbildung 5.2-2: Dienstewettbewerb}

Zusätzlich besteht die Möglichkeit, einzelne Netzelemente individuell pro durchgeleiteten Dienst aber auch dauerhaft furr einen Zeitraum zu einem monatlich fixen Betrag von dem Newcomer anzumieten. Ein Sonderfall hierbei ist das Anmieten der Anschlußleitungen im Sinne des Full Unbundling. ${ }^{61}$ Die Bestimmung des Zugangsentgelts kann pro telefonierter Einheit erfolgen oder aber als monatlicher Mietpreis für einen Teilnehmeranschluß.

\subsubsection{ERMITTLUNG EINES ZUGANGSENTGELTS NACH DEN GRENZ- KOSTEN BZW. NACH DEN INKREMENTELLEN KOSTEN}

Die Höhe des Zugangsentgelts $a$ ergibt sich aus der Differenz des Endproduktpreises, den der Newcomer veranschlagt, und seinen Grenzkosten für die Bereitstellung von Diensten:

$$
a=p_{2}-c_{2} .
$$


Da gemäß Gleichung (5.2.5) $p_{2}-c_{2}=2 c_{0}$ ist, folgt aus den Gleichungen (5.2.5) und (5.2.6) zunächst:

$$
a=2 c_{0} .
$$

Ein Zugangsentgelt in dieser Höhe würde dem Incumbent gerade die Grenzkosten für die Gewährung eines Zugangs zum Netz decken. Da hier eine marginale Einheit betrachtet wird, stimmen die inkrementellen Kosten mit den Grenzkosten überein. Er würde bei einer Bereitstellung des Netzzugangs zu Grenzkostenpreisen Verluste machen, weil zum einen seine infrastrukturell bedingten hohen Ausgaben dabei nicht berücksichtigt werden. Zum anderen handelt es sich um ein Mehrproduktunternehmen, bei dem Gemeinkosten anfallen, die bei Grenzkostenbzw. Inkrementalkosten-Preisen nicht abgedeckt werden. Es gäbe keinen Anreiz für ihn, einem neuen Wettbewerber auf dem Dienstemarkt Zugang zu vorgelagerten Märkten zu gewähren. Die Grenzkostenpreisregel ist folglich für die Bestimmung eines Zugangsentgelts im Hinblick auf die Einfuhrung von Wettbewerb nicht optimal. Für die Bestimmung eines optimalen Zugangsentgelts, das den hohen Fix- und Gemeinkosten, die der Incumbent bereits getätigt hat, gerecht wird, müßte auf die Grenzkosten ein Preisaufschlag erfolgen:

$$
a=2 c_{0}+\text { mark }-u p \text {. }
$$

Im Folgenden soll es darum gehen, wie dieser Preisaufschlag bestimmt werden soll. Dabei soll von Bedeutung sein, wie niedrig oder hoch der Preisaufschlag sein kann, damit erstens ein Markteintritt effizienter Wettbewerber in den nachgelagerten Markt erfolgt und zweitens der Incumbent keine Verluste durch die Gewährung von Netzzugang erleidet. Der Aufschlag sollte zu einem Zugangsentgelt führen, bei dem der Incumbent indifferent gegenüber einem Markteintritt ist.

\subsubsection{ERMITTLUNG EINES ZUGANGSENTGELTS NACH DER RAMSEY- PreisRegel}

$\mathrm{Da}$ es sich bei dem Incumbent um einen Mehrproduktmonopolisten handelt, der sowohl auf den vorgelagerten als auch auf den nachgelagerten Märkten Produkte bereitstellt, macht es Sinn, die Ramsey- Regel in bezug auf die Ermittlung eines Zugangsentgelts anzuwenden. ${ }^{62}$ Die Ramsey- Regel liefert eine Möglichkeit zur Bestimmung des Preisaufschlags und wurde in Kapitel 5.1.5 allgemein vorgestellt.

${ }_{62}^{61}$ Vgl. dazu Kap. 3.2.2.

62 Eben weil es sich um ein Mehrproduktunternehmen handelt, kommt eine Preisregel auf der Grundlage von Durchschnittskosten nicht in Betracht. 
Diese Preisregel hat zum Ziel, die Gemeinkosten des Incumbents zu decken, um zu verhindern, daß der Incumbent keine Verluste macht. Dann würde er sich einem Markteintritt gegenüber indifferent verhalten und wirde so $\mathrm{zu}$ der Einfuhrung von Wettbewerb auf dem nachgelagerten Markt beitragen.

Die Anwendung der Ramsey- Preisregel hinsichtlich der Bestimmung des Preisaufschlags impliziert die Berücksichtigung der Konsumentenseite neben der Seite der Anbieter. Es wird eine Wohlfahrtsmaximierung zugrunde gelegt. ${ }^{63}$ Die Wohlfahrt $W$ ergibt sich aus der Maximierung der Konsumentenrenten und Produzentenrenten, die sich wie folgt ermitteln läßt:

$$
W=S(X)-K_{l} \rightarrow \max X
$$

wobei sich die Brutto-Konsumentenrente aus der Addition beider nachgefragter Dienste ergibt:

$$
S(X)=\int p_{1}\left(x_{1}\right) d x_{1}+\int p_{2}\left(x_{2}\right) d x_{2} \cdot^{64}
$$

Gleichung (5.2.8) soll maximiert werden unter der Nebenbedingung, daß der Incumbent keine Verluste hinnehmen muß: $\Pi_{I} \geq 0$,

$$
\Pi_{1}=x_{1}\left(p_{1}-c_{0}^{2}-c_{1}\right)+x_{2}\left(p_{2}-c_{0}^{2}-c_{2}\right)-k_{l} .
$$

Der erste und zweite Term ergeben sich aus den Erträgen und Kosten aus der Bereitstellung von Diensten, die entweder der Incumbent selbst oder der Entrant am Endproduktmarkt vertreiben abzüglich der getätigten infrastrukturbedingten Kosten im vorgelagerten Markt $\left(k_{l}\right)$.

Auf der Suche nach dem optimalen Zugangsentgelt wird die Maximierung nach $x_{2}$ vollzogen, weil der Newcomer Zugang zum vorgelagerten Markt benötigt:

$$
\frac{\partial W}{\partial x_{2}}=p_{2}-2 c_{0}-c_{2}+\lambda\left[\frac{\partial p_{2}}{\partial x_{2}} x_{2}+p_{2}-2 c_{0}-c_{2}\right] \text {. }
$$

Nach einigen Umformungen und einer Erweiterung kommt man zu dem Ergebnis $^{65}$ :

$$
\frac{p_{2}-c_{2}}{p_{2}}=2 c_{0}+\frac{\lambda}{1+\lambda} \frac{1}{\eta_{p_{2}, x_{2}}}
$$

63 Dieses Konzept ist grundsätzlich angreifbar. Vgl. Kap. 5.1.5 sowie Kap. 6.

64 Es wird eine lineare Nachfragefunktion angenommen.

os In Anlehnung an Kap. 5.1.5. 
Dann ergibt sich nach der Definition des Zugangsentgelts gemäß der Gleichungen (5.2.5), (5.2.6) und (5.2.7) folgendes Ramsey- optimale Zugangsentgelt $a$ für die Bereitstellung des Dienstes $x_{2}$ :

$$
a=2 c_{0}+\frac{\lambda}{1+\lambda} \frac{p_{2}}{\eta_{p_{2}, x_{2}}} .66
$$

Auf die entstehenden Grenzkosten für die Netzzugangsgewährung $\left(2 c_{0}\right)$ wird neben dem $\lambda$-Term noch die mit dem Wiederverkaufspreis bewertete inverse Preiselastizität der Nachfrage $\eta_{p_{2}, x_{2}}$ aufgeschlagen. Die Berücksichtigung der Nachfrageelastizität hat zur Folge, daß die Konsumentenseite bei der Bestimmung des Zugangsentgelts mit einbezogen wird. Die Höhe des Zugangsentgelts ist davon abhängig, wie elastisch die Konsumenten auf Preisveränderungen reagieren. Ist die Nebenbedingung (5.2.9) nicht bindend, d.h. $\lambda / 1+\lambda=0$, dann wäre davon auszugehen, daß der Incumbent Verluste hinnehmen muß. Unter solchen Umständen würde sich ein Zugangsentgelt ergeben, das gerade die Grenzkosten deckt, also $a=2 c_{0}$. Da hier aber die break-even Bedingung bindend für das Erreichen eines Optimums sein soll, ist $\lambda / 1+\lambda>0 .^{67}$ Daraus folgt, daß das Zugangsentgelt über den Grenzkosten liegen muß, also $a>2 c_{0}$.

Unter der Annahme konstanter Grenzkosten und auf den hier relevanten Produktmärkten voneinander unabhängiger Nachfragefunktionen ergibt sich entsprechend der inversen Elastizitätsregel: Je weniger elastisch die Konsumentennachfrage im nachgelagerten Markt reagiert, desto höher kann das Zugangs- bzw. Zusammenschaltungsentgelt sein, d.h. desto höher wird der Incumbent für die Gewährung von Netzzugang pro vermitteltes Gespräch entschädigt. Je elastischer die Nachfrage nach Diensten ist, desto geringer sollte der Preisaufschlag auf die Grenzkosten für die Gewährung von Netzzugang sein. Entscheidend ist bei dieser Preisregel, daß die Höhe des Zugangsentgelts unter anderem von der Reaktion der Konsumenten auf Preisveränderungen gemacht wird.

Als Fazit läßt sich folgendes festhalten: Ein optimales Zugangsentgelt liegt nach der Ramsey- Preisregel bei einer statischen Analyse im Hinblick auf allokative und produktive Effizienz über den Grenzkosten. ${ }^{68} \mathrm{Da}$ die Entgelthöhe von der Nachfrageelastizität abhängig gemacht wird, lassen sich keine eindeutigen Aussagen darüber machen, inwieweit die Ramsey- Preisregel den Wettbewerb fördert.

\footnotetext{
66 Vgl. Arnbak u.a. (1994), S. 75; Mitchell u.a. (1994), S. 98; Laffont/Tirole (1994b), S. 1676 ff. und (2000), S. 102 ff.. Das Ergebnis in Laffont/Tirole (1996), S. 241 weicht geringfügig nicht vom Grundsatz her - ab.

$67 \mathrm{Vgl}$. Armstrong/Doyle/Vickers (1996) S. 135.

68 Vgl. zur grundsätzlichen Kritik an der Ramsey- Preisregel Kap. 5.1.5.
} 


\subsubsection{ERMITLUNG EINES ZUGANGSENTGELTS NACH DER ECPR}

Im Folgenden wird eine Einigung auf ein Zugangsentgelt gemäß der EfficientComponent-Pricing-Rule (ECPR), auch bekannt als Paritäts-Preis-Prinzip oder als Baumol-Willig-Regel, analysiert. ${ }^{69}$ Demnach muß das optimale Zugangsentgelt in der Höhe gestaltet sein, daß der Mieter dem Netzvermieter neben den direkt entstandenen Kosten für die Gewährung des Netzzugangs die anfallenden Opportunitătskosten für die Bereitstellung des Netzzugangs zahlt. Die direkt entstandenen Kosten sind die relevanten Kosten, die für die Bereitstellung des Netzzugangs anfallen. Baumol/Willig interpretieren sie als durchschnittliche inkrementelle Kosten im Rahmen der Theorie bestreitbarer Märkte. ${ }^{70} \mathrm{Da}$ im folgenden eine marginale Einheit betrachtet wird, entsprechen die inkrementellen Kosten den Grenzkosten. Die Opportunitätskosten bestehen hier aus dem Gewinnentgang des Netzvermieters, nämlich den Diensteeinheiten, die der Entrant nach Zugang zum Netz verkaufen konnte, der mit einem Nachfragerückgang für Gütereinheiten auf dem nachgelagerten Markt für den monopolistischen Incumbent einhergeht. Das $\mathrm{Zu}$ gangsentgelt ergibt sich aus der Differenz zwischen dem Verkaufspreis fur die Dienstleistung, die der Incumbent bereitstellt, und den Kosten, die der Incumbent durch den Wiederverkauf des Entrants hat.

Angenommen, der Entrant leitet eine Diensteeinheit des Incumbents weiter. Das kostet den Incumbent den entgangenen Stückgewinn plus den Grenzkosten für die Gewährung von Netzzugang ${ }^{71}$

$$
a=p_{1}-\left(2 c_{0}+c_{1}\right)+2 c_{0} .
$$

Das Zugangsentgelt $a$ entspricht dem Inputpreis des Incumbents $p_{\text {Input, }}$ aus Kapitel 5.1.6 . Der Endproduktpreis $p_{\text {End, }}$ ist hier mit $p_{1}$ gekennzeichnet. In den relevanten Inputkosten für den Incumbent sind die durch die Zugangsgewährung des Wettbewerbers zum Netz anfallenden Kosten $2 c_{0}$ und die Opportunitätskosten - dem entgangenen Stückgewinn - enthalten. Daraus ergibt sich nach der ECPR folgendes Zugangsentgelt:

$$
a=p_{1}-c_{1} \text {. }
$$

Ein Zugangsentgelt auf Basis der ECPR entspricht einem Preis, zu dem der Incumbent freiwillig einen Netzzugang furr seine Konkurrenten bereitstellt. Da er bei diesem Preis keine Verluste hinnehmen muß bzw. ausreichend für die Netzzugangsgewährung (Vermietung seiner Netzstruktur) kompensiert wird, ist er indif-

\footnotetext{
69 Vgl. Willig (1979), Baumol (1983), Baumol/Sidak (1994a,b) und Kap. 5.1.6.

$70 \quad$ Vgl. Kap. 5.1.3.

7 Vgl. Laffont/Tirole (1996), S. 237 ff. und (2000), S. $119 \mathrm{ff}$.
} 
ferent in bezug auf die Durchleitung von Telefongesprächen von wettbewerblichen Anbietern.

Die ECPR kommt unter der in Kapitel 5.1.6 erläuterten Annahmestruktur zu demselben Ergebnis wie die Ramsey- Preisregel gemäß den Gleichungen (5.2.6) und $(5.2 .14) .^{72}$

$$
p_{1}-c_{1}=p_{2}-c_{2}=a \text {. }
$$

D.h. die ECPR erzielt unter diesen Umständen sowohl produktive als auch allokative Effizienz. Die ECPR erreicht allokative Effizienz nur bei den unterstellten Annahmen, die sehr restriktiv sind. Dagegen ist das Ergebnis der produktiven Effizienz recht robust. Die Regel unterstützt den Markteintritt produktiv effizienter Unternehmen, und gleichzeitig reduziert sie nicht die Anreize des Netzbetreibers, seine Infrastruktur $\mathrm{zu}$ warten und weiter zu verbessern. Sie ermutigt Newcomer, Investitionen in Bypassing und infrastrukturellen Netzaufbau zur Überwindung des Bottlenecks zu tätigen. Außerdem sind die regulatorischen Anforderungen minimal bzw. gleich Null.

Die ECPR hat in der wirtschaftspolitischen Praxis folgende Rolle gespielt: Sie wurde als Preisregel für den Wiederverkäuferwettbewerb ${ }^{73} \mathrm{im}$ amerikanischen Telekommunikationsmarkt vorgeschlagen.

1992 schlug der Direktor der englischen Regulierungsbehörde Oftel Bryan Carsberg eine an die ECPR angelehnte Preisregel vor, die ,Oftel rule' oder auch ,Access Deficit Contribution Rule' (ADC) genannt wird. ${ }^{74}$ Die Regel wich in folgenden Punkten von der ECPR ab: Erstens wurde die ,Fully Distributed Costs Methode (FDC) ${ }^{75}$ angewendet, um die Grenzkosten im Bottleneck bzw. im vorgelagerten Markt bestimmen zu können. Zweitens sollte der Newcomer 50\% des Zugangsdefizits mittragen, weil sich die entstehenden Kosten für seine Zugangsgewährung nicht konkreter bestimmen ließen. Drittens wurde die ,Rate of Return“ Methode angewendet. Viertens sollte die Regulierungsbehörde einen stärkeren Einfluß behalten, als es die ECPR vorsah. Eine Regulierungsbehörde sollte den ,Fair Return' bestimmen. Außerdem sollte sie die Befugnis haben, einem neuen Wettbewerber den Verzicht auf die Beteiligung am Zugangsdefizit zu erlassen. Die volle ADC sollte nur gezahlt werden, wenn der ehemalige Monopolbetreiber einen Marktanteil von mehr als $85 \%$ hatte. Die ADC konnte wiederum abgesenkt werden, wenn der Incumbent (British Telecom) den Eckwert nicht erreichte. AuBerdem sollte die $\mathrm{ADC}$ nur für internationale Telefongespräche gezahlt werden.

\footnotetext{
72 Vgl. Laffont/Tirole (2000) S. $122 \mathrm{f}$.

73 Vgl. Laffont/Tirole (2000), S. 23.

74 Näheres über ihre Konstruktion und Funktionsweise vgl. Laffont/Tirole (2000), S. 168 f., Valletti (1999), S. 22 f.

75 Zur FDC vgl. auch Kap. 5.4.
} 
Die Möglichkeit des Verzichts der $\mathrm{ADC}$, die starken Einschränkungen und die Absicherung regulatorischer Eingriffe gegenulber der ECPR fuhrte dazu, daß die Regel $\mathrm{zu}$ Marktverzerrungen und Ineffizienzen fuhrte. Letztendlich spielte die Oftel-Rule in der Praxis keine große Rolle und wurde 1996 vollständig aufgegeben. $^{76}$

Nicht zuletzt sei auf die Auseinandersetzung zwischen dem Incumbent Telecom New Zealand, der die ECPR als angemessene Preisregel vorgeschlagen hatte, und dem Newcomer Clear verwiesen, der die Preisregel nicht akzeptieren wollte. ${ }^{77}$ Clear trat 1990 in den Markt für Ferngespräche ein und ersuchte ein Abkommen über den Zugang zum Teilnehmeranschlußbereich. Telecom nahm die ökonomische Beratung von Baumol und Willig in Anspruch und schlug die ECPR zur Regelung des Zugangs zum Ortsnetz vor. Clear war mit der Höhe des Zugangsentgelts nicht einverstanden und ging vor Gericht. Der ,High Court' entschied im Dezember 1992, daß die ECPR nicht gegen $\$ 36$ des Commerce Aktes ${ }^{78}$ verstoße. Clear ging in Berufung. Der ,Court of Appeal' gab im Dezember 1993 Clear Recht, der Incumbent könne nicht per Gesetz ein Zugangsentgelt festlegen, welches eine Monopolrente beinhalte. Daraufhin wendete sich Telecom an die höchste Instanz, das juristische Komitee des ,Privy Council'. Das ,Privy Council" (1994) entschied, daß die ECPR nicht wettbewerbsfeindlich sei, sondern ein wettbewerbliches Umfeld auf einem ,level playing field' schaffen würde. Das nach der ECPR ermittelte Zugangsentgelt sei nicht so hoch, daß es Clear an einem Markteintritt hindern würde. Dennoch kam es nicht zur Anwendung der ECPR. Die neuseeländische Regierung forderte die Parteien auf, sich auf einen Preis unterhalb der ECPR zu einigen. Der neuseeländische Fall hat insofern die Aufmerksamkeit erregt, weil das höchste Gericht erstmals darüber befunden hatte, daß die ECPR eine angemessene und wettbewerbsfördernde Regel sei.

\footnotetext{
76 Vgl. Oftel (1994).

77 Vgl. Baumol/Sidak (1994a), S. 189 ff.; Carter/Wright (1999), S. 241 ff.; Ministry of Commerce an the Treasury of New Zealand (1995), S. 25 ff.

$78 § 36(1)$ des Commerce Acts: „No person who has a dominant position in a market shall use that position for the purpose of (a) Restricting the entry of any person into that or any other market: or (b) Preventing or deterring any person from engaging in competitive conduct in that or any other market: or (c) Eliminating any person from that or any othe market. Commerce Act (1986), No. 5, §36 N.Z. Stat. 71, 95.
} 


\subsubsection{ERMITTLUNG EINES ZUGANGSENTGELTS BEI ABHÄNGIGEN NACHFRAGEN}

Bislang wurde bei den bisher angewendeten Preisregeln unterstellt, daß die Nachfragen nach Diensten verschiedener Anbieter voneinander unabhängig sind. Wird diese Annahme aufgegeben, eröffnet dies die Möglichkeit zu beobachten, inwieweit Preisveränderungen eines Dienstes die Nachfrage nach Diensten des Wettbewerbers beeinflussen. Dabei soll analysiert werden, welchen Einfluß nachfrageseitige Substitutionsmöglichkeiten auf die Höhe des Zugangsentgelts haben.

Die Möglichkeit, ein Zugangsentgelt bei voneinander abhängigen Nachfragen zu bestimmen, besteht in einer kombinierten Preisregel, die Elemente aus der Ramsey- und der EC- Preisregel enthält, und die zudem das Substitutionsverhältnis zweier Dienste in Form einer Variable berücksichtigt, nämlich mit Hilfe eines Produktdifferenzierungsparameters, der an die ECPR gekoppelt ist. ${ }^{79}$ Als optimale Zugangspreisregel ergibt sich die ECPR plus einen Preisaufschlag nach der Ramsey-Regel. Mit der Preisregel wird gezeigt, inwieweit Produktdifferenzierung die Höhe des Zugangsentgelts beeinflussen kann. ${ }^{80}$ Die Möglichkeit, zwischen Diensten unterschiedlicher Anbieter zu substituieren, reduziert den Gewinnentgang und vermindert folglich die Opportunitätskosten des Zugangs, dessen Höhe an einem Verdrängungsverhältnis $\delta$ (displacement ratio) aufgezeigt wird.

$$
a=2 c_{0}+\frac{\lambda}{1+\lambda} \frac{p_{2}}{\eta_{p_{2}, x_{2}}}+\delta\left(p_{1}-2 c_{0}-c_{1}\right), \quad \text { wobei } \delta=-\frac{\frac{\partial x_{1}}{\partial p_{2}}}{\frac{\partial x_{2}}{\partial p_{2}}} .
$$

Das neu ermittelte Zugangsentgelt besteht aus drei Komponenten: erstens aus den Grenzkosten für die Gewährung von Netzzugang $2 c_{0}$. Auf die Grenzkosten werden zwei Preisaufschläge berechnet: Der eine Preisaufschlag ist von einer gewöhnlichen Nachfrageelastizität abhängig, die mit Hilfe der Ramsey- Preisregel ermittelt wird. Dieser entspricht dem zweiten Term der Gleichung (5.2.16) und

79 Vgl. hierzu den Artikel von Armstrong/Doyle/Vickers (1996). Eine andere Möglichkeit besteht darin, die herkömmliche Nachfrageelastizität bei der Ramsey- Preisregel durch eine Superelastizität zu ersetzen. Vgl. hierzu Laffont/Tirole $(1994,1996,2000)$, die das Basismodell um modifizierte Nachfrageelastizitäten erweitern.

BO

Armstrong/Doyle/Vickers (1996), S. 136 ff. erweitern ihre Analyse bezüglich der displacement ratio neben der Berücksichtigung von Produktdifferenzierung zusätzlich auf Bypassmöglichkeiten ( $=$ angebotsseitige Substitutionsmöglichkeiten) und Inputsubstitutionsmöglichkeiten. In meiner Analyse werden diese Aspekte in bezug auf den One-Way-Access außer acht gelassen, weil sie sich per definitionem ausschließen. Insbesondere auf den Bypass wird allerdings in Kap. 5.3 bei der Analyse von infrastrukturbasiertem Wettbewerb explizit eingegangen werden. 
berücksichtigt eine differenzierte Nachfrage als Reaktion auf eine Preisveränderung. ${ }^{81}$ Der zweite Preisaufschlag und damit die dritte Komponente besteht aus dem Gewinnentgang, die dem Incumbent durch die Gewährung von Netzzugang entsteht, bewertet mit einem Substitutionsverhältnis $(\delta)$ zwischen Wiederverkaufs- und Zugangsaktivitäten des Incumbents. Dieses Substitutions- oder Verdrängungsverhältnis stellt den Rückgang der Nachfrage nach Diensten, die der Incumbent bereitstellt $\left(x_{1}\right)$, dar, die durch die Gewährung des Zugangs des Entrants bedingt ist $\left(x_{2}>0\right)$. Es handelt sich hierbei um den Gewinnverlust des Incumbents bedingt durch das Angebot einer marginalen Einheit an Zugang an den neuen Wettbewerber. Das Verdrängungsverhältnis soll zwischen Null und Eins liegen $[0 \leq \delta \leq 1]$ und den Grad an Produktdifferenzierung widerspiegeln. Gibt es beispielsweise keine Möglichkeit, zwischen den Gütern zu substituieren, dann verändert sich die Nachfrage nach den Diensten des Incumbents nicht, d.h. $\delta=0$. Dann entspräche die Höhe des Zugangsentgelts der Ramsey- Preisregel. Handelt es sich dagegen um homogene Produkte, d.h. die von unterschiedlichen Betreibern bereitgestellten Dienste werden als perfekt substitutierbar empfunden, dann gilt: $|\delta|=1$. Es ergäbe sich folgendes Zugangsentgelt:

$$
a=p_{1}-c_{1}+\frac{\lambda}{1+\lambda} \frac{p_{2}}{\eta_{p_{2}, x_{2}}} \text {. }
$$

Das hieße, daß auf die ECPR zusätzlich noch der Ramsey- Term aufgeschlagen würde. ${ }^{82}$

Für $|\delta| \rightarrow 0$ gilt: je weniger die Produkte als Substitute empfunden werden bzw. je größer die Produktdifferenzierung ist, desto kleiner ist das Verdrängungsverhältnis. Eine angebotene Einheit eines Rivalen fuihrt nicht zwangsläufig zu einem Nachfragerückgang für den Incumbent. Daraus folgt, daß das Zugangsentgelt kleiner sein kann als bei homogenen Produkten. ${ }^{83}$

Je mehr die angebotenen Dienste von den Konsumenten als Substitute empfunden werden, desto größer ist das Verdrängungsverhältnis; $|\delta| \rightarrow 1$. Daraus folgt ein höheres Zugangsentgelt im Vergleich zu homogenen Produkten, weil dem Incumbent Nachfrage an den konkurrierenden Diensteanbieter verloren geht und damit sein Gewinnentgang zunimmt. Neben den anfallenden Opportunitätskosten für den Incumbent wird zusätzlich noch die Preiselastizität der Konsumenten mit einbezogen. Die Berücksichtigung des substitutiven Verhältnisses zwischen den von

\footnotetext{
81 Vgl. Valletti/Estache (1999), S. 21.

82 Valletti und Estache (1999) wenden allerdings gegen diesen Fall ein, daß es keine Verdrängung im 1:1 Verhältnis gibt, d.h. $\delta$ ist nicht gleich 1. Vgl. Valletti/Estache (1999), S. 21.

83 Dieser Modellierungsansatz kommt in bezug auf den Einfluß der Berücksichtigung substitutiver Produkte auf die Bestimmung des Zugangsentgelts im Grunde zu demselben Ergebnis wie die Berilcksichtigung von Su-perelastizităten bei der Ramsey- Preisregel.
} 
den beiden unterschiedlichen Anbietern bereitgestellten Diensten führt in jedem Fall zu einer Reduktion der Opportunitätskosten, weil die Nachfrage der Konsumenten preiselastischer reagiert. Dementsprechend erhöht sich der Wettbewerbsdruck auf beide Anbieter. Für den Incumbent hat das zur Folge, daß seine Einnahmen im kompetitiven nachgelagerten Markt geschmälert werden. Es kann dazu kommen, daß er seine Fix- und Gemeinkosten nicht mehr decken kann und er einen Verlust macht. Aus diesem Grund ist es zu rechtfertigen, daß unter diesen Umständen das Zugangsentgelt höher als bei der ECPR in Kapitel 5.2.3 zu setzen ist, als unter der Annahme, daß die Nachfragen voneinander unabhängig sind.

\subsubsection{FAZIT: VeRgLeICH DER PREISREgelN IM HiNBliCK AUF DIE EINFÜHRUNG VON WETTBEWERB}

Abschließend läßt sich zu der anfangs in Kapitel 5 formulierten Frage, inwieweit sich die einzelnen Preisregeln für die Ermittlung eines Zugangsentgelts eignen, um Wettbewerb auf dem nachgelagerten Markt einzuführen, folgendes sagen. Es gibt theoretische Konzepte, nach denen Preisregeln ermittelt werden können, die zu ökonomischer Effizienz führen. Die hier vorgestellten Preisregeln sind so konzipiert, daß sie auf unterschiedliche Art einen Preisaufschlag bestimmen, der zu einem Grenzkostenpreis hinzugerechnet werden soll. Die Ramsey- Regel basiert auf einem wohlfahrtsökonomischen Ansatz. Ihr besonderes Markenzeichen ist, daß sie die Nachfragerseite bei der Bestimmung des Zugangsentgelts mit berücksichtigt. Sie macht es sich zur Aufgabe, ein allokativ effizientes Ergebnis zu erzielen. Für die ECPR stehen bei der Bestimmung eines Zugangsentgelts ausschließlich die Anbieter im Vordergrund. Sie zielt auf ein produktiv effizientes Ergebnis ab. Je nachdem, wie die Annahmen sind, kann das Ergebnis auch allokativ effizient sein. Die ECPR ist in jedem Fall die praktikablere Lösung als die Ramsey- Regel. In der wirtschaftspolitischen Praxis ist die ECPR mehrfach diskutiert worden ist. Wie in der wirtschaftspolitischen Praxis nun tatsächlich die Zugangsentgelte bestimmt werden, wird in Kapitel 5.4 näher analysiert. Die Berücksichtigung voneinander abhängiger Nachfragen läßt eine differenziertere Analyse hinsichtlich der Bestimmung des Zugangsentgelts zu. Dann nämlich ist die Höhe des Zugangsentgelts von der Substituierbarkeit der bereitgestellten Dienste im nachgelagerten Markt abhängig.

Die von der Regulierungsbehörde rechtlich vorgegebene Entgeltregulierung, einen Netzzugang an den Kosten der effizienten Leistungsbereitstellung zu orientieren, läßt einen gewissen Interpretationsspielraum zu. Orientiert die Regulierungsbehörde ihre Entgeltvorgabe an der These, je niedriger das Zugangsentgelt ist, desto 
förderlicher ist es furr einen Markteintritt in den Dienstemarkt, ist diese Politik fragwürdig. Sie würde in jedem Fall zu einer Stärkung des Wiederverkäufer- und Dienstewettbewerbs fuhren. Gleichzeitig würde sie aber den infrastrukturbasierten Wettbewerb schwächen. Je niedriger das Zugangsentgelt ist, desto geringer ist der Anreiz fur alternative Anbieter, eine eigene Netzinfrastruktur aufzubauen, der zu einer Verzerrung der tatsächlichen Marktsituation führt und falsche Anreize schafft. Eine Förderung des Wiederverkäufer- und Dienstewettbewerbs ist auf lange Sicht nicht sinnvoll, weil die Abhängigkeit zum vorgelagerten Markt bestehen bleibt und folglich alternative Anbieter sich immer in der Hand des Netzbetreibers befinden und sich nur unter dem Schutz regulierender Maßnahmen entfalten können.

Wenn man wirtschaftspolitisch den infrastrukturbasierten Wettbewerb fördern will, um damit langfristig Wettbewerb im Telekommunikationsmarkt zu stärken und zu stabilisieren, dann sollte das Zugangsentgelt die wahren Kosten gemäß der ECPR unter Berücksichtigung von Produktdifferenzierung widerspiegeln, auch wenn dadurch der Wiederverkäufer- und Dienstewettbewerb geschwächt würde.

\subsection{VERHANDLUNGEN VON ZUSAMMENSCHALTUNGS- ENTGELTEN BEI EINEM TWO-WAY-ACCESS}

Nachdem im vorangegangenen Kapitel die Bedeutung des Zugangsentgelts bei Wettbewerb im nachgelagerten Markt analysiert worden ist, sollen in diesem Kapitel die Auswirkungen von infrastrukturbasiertem Wettbewerb in bezug auf die Bestimmung von reziproken Zugangsentgelten bzw. Zusammenschaltungsentgelten fokussiert werden. Es handelt sich folglich um Anbieter, die sowohl als Netzbetreiber in den vorgelagerten Markt eintreten als auch Dienste über ihre eigene Infrastruktur im nachgelagerten Markt vertreiben. Hierbei soll sich insbesondere im Rahmen des Two-Way-Access auf Netzbetreiber konzentriert werden, die im Teilnehmeranschlußbereich um Kunden konkurrieren. Dies betrifft zum Beispiel substitutive Anbieter wie TV-Kabel- oder PLC- Betreiber, die nach erfolgreicher Umrüstung uber ein eigenes Teilnehmeranschlußnetz verfügen. Dabei wird davon ausgegangen, daß jeder Kunde sich nur einen Anschluß von jeweils einem Anbieter leistet, d.h. jeder Teilnehmeranschluß wird monopolisiert. Allerdings soll ein Wechsel zu einem konkurrierenden Anbieter ohne Wechselkosten und ohne technischen und zeitlichen Aufwand möglich sein. Außerdem wird eine Zusammenschaltungspflicht zwischen den Netzbetreibern unterstellt. 
Im Folgenden soll es um die Verhandlungen zwischen den Netzbetreibern in bezug auf das Zusammenschaltungsentgelt gehen ${ }^{84}$ und inwieweit sie sich auf die Höhe des Zusammenschaltungsentgelts und damit auf die Endproduktpreise auswirken. In diesem Zusammenhang soll auf Anreize über Absprachen seitens der Anbieter eingegangen werden und ob eine Regulierung der Zusammenschaltungsentgelte gegebenenfalls erforderlich ist. In Kapitel 5.3.1 wird zunächst an einem einfachen Modell die Bill-and-Keep- Regel vorgestellt, bei der sich Netzbetreiber gegenseitig kein Entgelt für die Zusammenschaltung in Rechnung stellen. Dabei soll analysiert werden, inwiefern Gleichgewichte, die auf der Bill-and-Keep- Regel basieren, stabil sind. $\mathrm{Da}$ in Kapitel 5.3.1 davon ausgegangen wird, daß die Anschlüsse verschiedener Betreiber von den Nachfragern als perfekte Substitute empfunden werden, soll im folgenden Kapitel 5.3.2 untersucht werden, inwiefern sich die Berücksichtigung einer begrenzten Substituierbarkeit zwischen den Anschlüssen verschiedener Betreiber auf die Verhandlung von Zusammenschaltungsentgelten auswirkt.

\subsubsection{BERTRAND- WETTBEWERB BEI VOLLSTÄNDIGER SUBSTITUIERBARKEIT}

In Abbildung 5.3-1 wird von infrastrukturbasiertem Wettbewerb zwischen zwei Anbietern $B=1,2$ ausgegangen. Es wird angenommen, daß es zwei Netzbetreiber mit einem eigenen Teilnehmeranschlußbereich gibt, der sich geographisch durchaus überschneiden kann. ${ }^{85}$

Dabei wird eine Situation dargestellt, in der sich keines der Netze noch im Aufbau befindet und die Konsumenten bereits Teilnehmer eines der beiden Netzwerke sind. Die Betreiber verfügen in diesem Modell über gleich große Netzwerke, d.h. sie verfügen über eine gleich hohe $Z$ ahl an Kundenanschlüssen. Es gibt für beide Anbieter einen hohen Anreiz, Zugang zu dem Netz des anderen Anbieters zu haben. Aufgrund der Annahme, daß die Netzwerke gleich groß sind, profitieren beide Seiten gleichermaßen von der Zusammenschaltung. ${ }^{86}$ Es existiert folglich eine glaubhafte Basis für den Erfolg einer Verhandlungslösung auf ein reziprokes $\mathrm{Zu}$ -

\footnotetext{
84 Einer Verhandlungslösung wird auch im TKG Priorität eingeräumt, obwohl in der Realität die Zusammenschaltungsentgelte faktisch einer Einzelpreisregulierung unterliegen. Vgl. dazu Kap. 2.4.3 sowie Kap. 5.4.

85 Die Kreise sollen BereichsVSts beider Wettbewerber skizzieren. Ihre unterschiedlichen Farben (schwarz/ weiß) kennzeichnen die Zugehörigkeit der VSt zu dem einen oder anderen Netzbetreiber.

86 Netzeffekte und Netzexternalităten können bestmöglich ausgeschöpft werden. Vgl. Kap. 4.
} 
gangsentgelt bzw. Zusammenschaltungsentgelt $a .^{87}$ Die Teilnehmeranschlußbereiche werden in der Abbildung 5.3-1 auf der Nahbereichsebene zusammengeschaltet. Die Teilnehmeranschlußbereiche sind anhand durchgezogener oder gestrichelter Linien erkennbar.
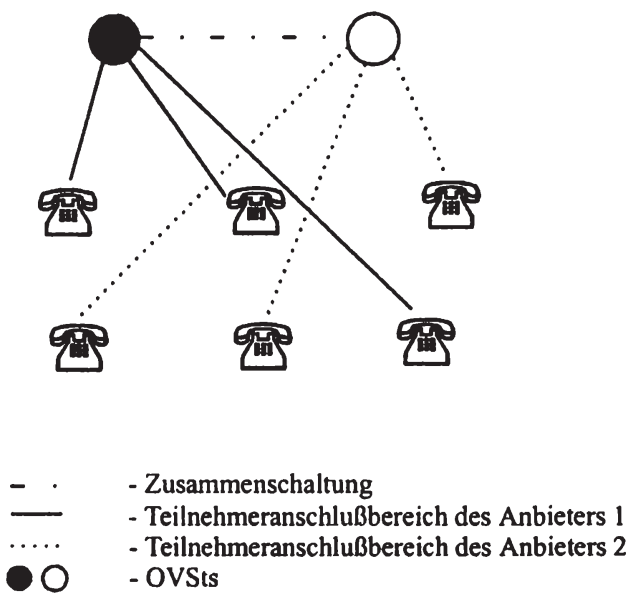

\section{Abbildung 5.3-1: Infrastrukturbasierter Wettbewerb}

Beide Betreiber bieten Telekommunikationsdienste an. Es handelt sich hierbei zunächst um ein homogenes Gut, das sich in der Qualität nicht unterscheiden soll. Dementsprechend lassen sich in dem hier dargestellten Modell die angebotenen Güter perfekt substituieren. Dabei wird vorausgesetzt, daß die Teilnehmer ohne Probleme von einem Anbieter zum nächsten wechseln können. Mit anderen Worten, es existieren keine Vertragslaufzeiten, und es gibt keine Switching- Costs in diesem Modell. Das reziproke Zugangsentgelt $a$ wird auf den Endproduktpreis aufgeschlagen. D.h. je höher das Entgelt, desto höher der Preis, den der Konsument pro Telefongespräch zahlen muß, nämlich $p_{1}$ oder $p_{2}$. Hier wird zunächst davon ausgegangen, daß der Endproduktpreis von den Anbietern in Reaktion auf die Wettbewerber und die Nachfrage der Konsumenten gesetzt wird. Außerdem soll hier immer der anrufende Konsument den in Anspruch genommenen Dienst zahlen. Weiterhin wird unterstellt, daß die Anbieter keine Preisdiskriminierung betreiben. In Anlehnung an Abbildung 5.3-1 wird angenommen, daß die Dienste

87 D.h. beide Netzbetreiber zahlen sich gegenseitig dasselbe Zusammenschaltungs- oder Interconnection- Entgelt. 
der Betreiber grundsätzlich im eigenen Netz originiert, aber sowohl im eigenen Netz als auch im Netz des Konkurrenten terminiert werden.

Für den Anbieter 1 fallen neben den Gemein- bzw. Fixkosten $k_{1}$ für die infrastrukturelle Bereitstellung zusätzlich Kosten für Dienste der Originierung und der Transitleistung $\left(c_{0}+c_{1}\right) x_{1}$ und der Terminierung im Netz des anderen Betreibers $\left(a x_{1}\right)$ bei Off-net- Telefongesprächen, der dafür ein Terminierungsentgelt bzw. Zusammenschaltungsentgelt verlangt, an. Die Kosten einer marginalen Telefoneinheit entsprechen $c_{0}+c_{1}+a .^{88}$

Der Anbieter 1 erwirtschaftet neben den Erlösen aus nachgefragten On-net- wie Off-net- Diensten seitens der Konsumenten $\left(p_{1} x_{2}\right)$ zusätzlich Erlöse aus der Terminierung von Diensten, die in dem Netz des alternativen Betreibers originiert wurden $\left(a x_{2}\right)$.

Der Gewinn eines Anbieters läßt sich dann folgendermaßen formulieren:

$$
\Pi_{1}=x_{1}\left(p_{1}-c_{0}-c_{1}-a\right)-k_{1}+a x_{2}
$$

bzw.

$$
\Pi_{2}=x_{2}\left(p_{2}-c_{0}-c_{1}-a\right)-k_{2}+a x_{1} \text {. }
$$

Grundsätzlich wird ein ,ausbalanciertes Telefoniermuster' unterstellt, das besagt: je größer das Netz ist, desto mehr Gespräche werden hinaus- und hineingeleitet. ${ }^{89}$ Die zentrale Frage soll in dem folgenden Abschnitt lauten, welchen Preis die Betroffenen für die Zusammenschaltung verhandeln werden. Als Argumentationsgrundlage wird auf das Bertrand- Paradox zurückgegriffen, das zu dem Ergebnis führt, daß sich zwei Anbieter ihre Endproduktpreise gegenseitig bis zu den Grenzkosten herunterkonkurrieren. Für die Verhandlung über das Zusammenschaltungsentgelt bedeutet die Anwendung der Bill-and-Keep- Regel, daß sich gegenseitige Zahlungen an Zusammenschaltungsentgelten auf Null saldieren.

Der zugrunde liegende Bertrand- Wettbewerb umfaßt simultanen und nicht kooperativen Preiswettbewerb zwischen zwei Anbietern. ${ }^{90}$ Vorgegangen wird nach einem Standard-Zwei-Phasen- Spiel. In der zweiten Phase, die aber analytisch zuerst betrachtet wird, bestimmen die Betreiber bei gegebenem $a$ die Höhe ihres Endproduktpreises nicht-kooperativ und nehmen den Preis des Konkurrenten als gegeben an. In der ersten Phase wird das Zusammenschaltungsentgelt $a$ von den Betreibern simultan gesetzt. Dabei wird unterstellt, daß $p_{B}$ dem nicht kooperativ gesetzten Wiederverkaufspreis der beiden Netzbetreiber $B=1,2$ bei zunächst gegebenem Zusammenschaltungsentgelt $a$ entspricht. Da es sich bei den von den

\footnotetext{
88 Zur analytischen Formulierung der bei einem Telefondienst anfallenden Grenzkosten vgl. auch Laffont/Tirole (1996), S. 232 f.; (1998), S. 6; (2000), S. $100 \mathrm{ff}$.

89

In Wirklichkeit kann es durchaus sein, daß sich ein Dienstleister mit sehr hohem Gesprächsaufkommen in einem kleinen Netz befindet, wie z.B. ein Call Center oder ein Versandhaus. Dann käme es dementsprechend hinsichtlich der hier getroffenen Aussagen zu Verzerrungen.

Vgl. Tirole (1995), S. $455 \mathrm{ff}$.
} 
Betreibern angebotenen Telekommunikationsdiensten um homogene Produkte handelt, folgt daraus, daß beide Betreiber eine einheitliche gesamtwirtschaftliche Nachfragefunktion aller On-net- und Off-net- Dienste im Blick haben: $X=x_{1}+x_{2}$. Hierbei gilt folgendes für die Preis-Absatz-Funktion des DiensteAnbieters 1 bzw. 2:

$$
x_{1}\left(p_{1}, p_{2}\right)= \begin{cases}X\left(p_{1}\right) & \text { für } p_{1}<p_{2} \\ 1 / 2 X\left(p_{1}\right) & \text { für } p_{1}=p_{2} \\ 0 & \text { für } p_{1}>p_{2}\end{cases}
$$

bzw. $\quad x_{2}\left(p_{2}, p_{1}\right)= \begin{cases}X\left(p_{2}\right) & \text { für } p_{2}<p_{1} \\ 1 / 2 X\left(p_{2}\right) & \text { für } p_{2}=p_{1} \\ 0 & \text { für } p_{2}>p_{1}\end{cases}$

Ihr wird unterstellt, daß ein Anbieter für den Fall, daß sein Endproduktpreis unter dem seines Konkurrenten liegt $\left(p_{1}<p_{2}\right.$ bzw. $\left.p_{1}>p_{2}\right)$, in der Lage ist, den gesamten Markt abdecken zu können, während der konkurrierende Betreiber vollkommen vom Markt verdrängt wird ( $x_{2}=0$ bzw. $\left.x_{1}=0\right)$.

Entsprechen sich die Endproduktpreise, wird der Markt auf beide Anbieter gleichmäßig aufgeteilt. Daraus ergibt sich gemäß der Gleichungen (5.3.1) und (5.3.2) folgende Gewinnfunktion für den Betreiber $B$ bei $p_{1}=p_{2}=p$ :

$$
\Pi_{B}=1 / 2 X(p)\left[p-c_{0}-c_{B}-a\right]+1 / 2 X(p) a-k_{B} .
$$

Der aggregierte Gesamtgewinn der beiden Betreiber kann den Monopolgewinn $\Pi_{M}$ eines Anbieters nicht ubersteigen: $0 \leq \Pi_{1}+\Pi_{2} \leq \Pi_{M}$.

Es wird angenommen, daß der Gewinn eines Unternehmens sowohl von seinem eigenen Preis als auch von dem Preis des anderen Unternehmens abhängt. Die Unternehmen legen in der zweiten Phase gleichzeitig und nicht-kooperativ ihre Preise fest. Es existiert ein Bertrand- bzw. Nash- Gleichgewicht bei $p_{1}^{*}, p_{2}^{*}$. Dabei gilt:

$\Pi_{1}\left(p_{1}^{*}, p_{2}^{*}\right) \geq \Pi_{1}\left(p_{1}, p_{2}^{*}\right)$ bzw. $\Pi_{2}\left(p_{1}^{*}, p_{2}^{*}\right) \geq \Pi_{2}\left(p_{1}^{*}, p_{2}\right)$.

Ein Bertrand-Gleichgewicht ist ein Gleichgewicht, bei dem jeder der beiden bei gegebener optimaler Strategie des anderen eine optimale Strategie furr sich selbst in bezug auf den Preis wählt. ${ }^{91}$

Bei Gewinnmaximierung von Gleichung (5.3.3) gilt

91 Vgl. Borrmann/Finsinger (1999), S. $68 \mathrm{ff}$. 


$$
\frac{\partial \Pi_{B}}{\partial p_{B}}=0
$$

Dann ergibt sich folgendes Preisgleichgewicht für Anbieter $B=1,2$ :

$$
\vec{p}_{n}^{\cdot}=\frac{c_{0}+c_{B}}{1-\frac{1}{\eta}} \quad \text { für } 1<\eta<\infty .
$$

Der hier ermittelte Endproduktpreis steht in Abhängigkeit zur inversen Nachfrageelastizität. Je preiselastischer die Nachfrage $(|\eta| \rightarrow \infty)$, desto mehr nähert sich der Nenner geht gegen 1. Ist die Nachfrage unendlich elastisch, kann man von Grenzkostenpreisen auf dem nachgelagerten Markt sprechen $\left(p_{B}=c_{0}+c_{B}\right)$. D.h. die hohe Preiselastizität der Teilnehmer löst einen Preisdruck auf die Anbieter aus, so daß sie gezwungen sind, Grenzkosten deckende Preise zu nehmen.

Nimmt die Preiselastizität $a b(|\eta| \rightarrow 1)$, werden die Endproduktpreise dementsprechend höher als die anfallenden Grenzkosten für die Zugangsgewährung sein $\left(p_{B}>c_{0}+c_{B}\right)$. Daraus folgt, je preisunelastischer die Nachfrager auf Preisveränderungen reagieren, desto eher sind Abweichungen von Grenzkostenpreisen nach oben hin möglich.

Angenommen, die Nachfrage reagiert unendlich preiselastisch und die Endproduktpreise differieren zunächst. Außerdem besteht eine gewisse Wechselfreudigkeit der Konsumenten, so daß sie zwischen den Anbietern den preiswerteren wählen. Daraus folgt, daß bei $p_{1}^{*}>p_{2}^{*}$ der Betreiber 2 einen preisbedingten Wettbewerbsvorteil hat. Der Anbieter 1 wird dem eine Preissenkung um eine marginale Einheit $\varepsilon$ unter dem Preis des Konkurrenten entgegensetzen: $p_{1}=p_{2}^{*}-\varepsilon$. Das wird wiederum den Carrier 2 dazu bewegen, ebenso mit seinem Preis herunterzugehen, bis er um $\varepsilon$ unter dem Preis des Konkurrenten liegt. Die Anbieter werden sich mit ihren Preisen so lange gegenseitig unterbieten, bis sie auf die Höhe der Grenzkosten gesunken sind. Es existiert folglich nur ein einziges BertrandGleichgewicht bei

$$
c_{0}+c_{B}=p_{1}^{\cdot}=p_{2}^{*} \cdot{ }^{92}
$$

An diesem Punkt machen Unternehmen keine Gewinne mehr. Es kommt trotz der oligopolistischen Ausgangslage zu einer Lösung, wie sie sich unter perfekten Wettbewerbsbedingungen ergeben würde. Dieses Konkurrenzverhalten bei zwei Anbietern wird auch als Bertrand- Paradox bezeichnet. Die Ursache für dieses Verhalten liegt in der Drohung für einen Anbieter, aus dem Markt vollständig

92 Dieses Ergebnis gilt unabhängig von der Zahl der Anbieter, auch wenn es hier nur am einfachsten Fall eines Duopols nachvollzogen wird. 
verdrăngt zu werden, wenn er nicht mit einer Preissenkung auf den Konkurrenten reagiert. Die implizit unterstellten Annahmen der vollständigen Markttransparenz und der Hypothese, daß die Unternehmen keinen Kapazitätsgrenzen unterliegen, führt dazu, daß der gesamte Markt jederzeit bei Austritt des konkurrierenden Anbieters von einem einzigen Unternehmen versorgt werden kann. Wenn sich die Betreiber nicht auf denselben Preis einigen, dann gibt es nur die Möglichkeit des totalen Marktaustritts oder der totalen Marktübernahme.

Nachdem die Endproduktpreise bestimmt worden sind, kann nun die Ermittlung des Zusammenschaltungsentgelts bei gegebenem $\vec{p}_{B}^{*}$ erfolgen:

$$
\begin{aligned}
& \Pi_{B}\left(p_{B}^{*}\right) \rightarrow \max \\
& \Pi_{B}=\left(\frac{c_{0}+c_{B}}{1+\frac{1}{\eta}}-c_{0}-c_{B}-a\right) 1 / 2 X(p)+a 1 / 2 X(p) \\
& \frac{\partial \Pi}{\partial a}=0 \\
& a=0 .
\end{aligned}
$$

Gilt das Bertrand- Paradox für den nachgelagerten Markt, dann bleibt den Betreibern kaum Spielraum für die Bestimmung des Zusammenschaltungsentgelts. $\mathrm{Da}$ hier unterstellt wurde, daß jeder Anbieter die Hälfte aller Dienste terminiert, saldieren sich die Grenzkosten und die Grenzeinnahmen aus der Zusammenschaltung für den jeweiligen Betreiber auf Null. Es kommt die Bill-and-Keep- Regel zum Tragen, nämlich daß sich die Carrier gegenseitig gar kein reziprokes $\mathrm{Zu}$ gangsentgelt bezahlen. Unter der Annahme, daß das Telefonaufkommen zwischen den beiden Netzen ausgewogen ist, könnte man annehmen, daß sich die gegenseitigen Zahlungen furr den Netzzugang auf Null saldieren und sich folglich Endproduktpreise auf Grenzkostenniveau ergeben. ${ }^{93}$

93 In Neuseeland haben das Ministery of Commerce und die Treasury eine Bill-and-Keep- Regel im Rahmen einer Two-Way-Interconnection befurwortet, sofern die Balance bezuiglich bereitgestellter Dienste zwischen den Netzwerkbetreibern gegeben ist. Dies bezieht sich sowohl auf die Netzwerkgröße als auch auf das ausgewogene Telefoniermuster. Vgl. Ministry of Commerce and the Treasury (1995), S. 88. Sobald das Telefonaufkommen zwischen den beiden Netzen nicht mehr ausgewogen ist, ist anzunehmen, daß sich die Grenzkosten und Grenzeinnahmen, die mit den Grenzkosten des Konkurrenten übereinstimmen, aus der Zusammenschaltung nicht mehr saldieren werden. Es ist zu erwarten, daß sich dann die Netzbetreiber furr den reziproken Netzzugang die relevanten Kosten, die bei der Bereitstellung des Netzzugangs anfallen, berechnen werden. Die relevanten Kosten können neben den tatsăch- 
Allerdings stellt die Bill-and-Keep- Regel bei perfekter Substituierbarkeit der angebotenen Telekommunikationsdienste und einer ausgewogenen Marktaufteilung kein stabiles Gleichgewicht dar, was sich wie folgt begründen läßt: Die Anreize eines Betreibers, Endproduktpreise zu erhöhen, hängen von drei Effekten ab. Der erste Effekt ist der Verlust an Marktanteilen bei einer Preiserhöhung, der deswegen dämpfend auf solche Anreize wirkt. Der zweite Effekt einer Preiserhöhung sind die zunehmenden Erträge aus dem Verkauf pro Konsument. Der dritte Effekt sind zunehmende Erträge aus der Zugangsgewährung im Falle einer Preiserhöhung. Dieser ist für die Konstellation von einem vor- und einem nachgelagerten Markt spezifisch und nimmt mit steigendem Zusammenschaltungsentgelt bei gleichen Marktanteilen zu. Das Zusammenschaltunsgentgelt ist eben keine neutrale Komponente, weil es in die wahrgenommenen Grenzkosten jedes Anbieters eingeht und zwar unabhängig davon, ob der Verkehr zwischen beiden Netzwerken ausgewogen ist und sich die Netto-Zahlungen gegenseitig ausgleichen.

Über die Verhandlung des Zusammenschaltungsentgelts haben beide Anbieter die Möglichkeit, die Kostenstruktur des anderen Anbieters zu beeinflussen. Wegen der Möglichkeit, auf die Kostenstruktur des anderen einzuwirken, besteht der Anreiz, die Kosten des Konkurrenten in die Höhe zu treiben, indem ein möglichst hohes Zusammenschaltungsentgelt verhandelt wird - der Kostenspiraleneffekt. ${ }^{94}$ Höhere Zusammenschaltungsentgelte haben bei gegebenem Marktanteil zur Folge, daß sich die Grenzkosten für bereitgestellte Dienste erhöhen und sich in höheren Endproduktpreisen niederschlagen. Das Ergebnis erscheint zunächst kontraintuitiv: Das Kalkül der Gewinnmaximierung kann nicht wirklich der Grund für den Kostenspiraleneffekt sein, weil ein erhöhtes Zusammenschaltungsentgelt zwar die Einnahmen aus der Zusammenschaltung erhöht, aber ebenso die Kosten aus der Zusammenschaltung ansteigen läßt. Und den Konkurrenten zu schädigen, kann auch nicht der dominante Grund sein, da der Anbieter sich ebenso selbst schädigt, indem er die Kosten in die Höhe treibt. Gleichwichtspreise bei verhandelten hohen Zusammenschaltungsentgelten sind sogar verhältnismäßig stabil. Senkt ein Betreiber beispielsweise den Endproduktpreis für Grunddienste, würde zunächst die Zahl an Off-net-calls zunehmen. Dann aber müßte der Betreiber auch eine höhere Summe an den Konkurrenten für die Durchleitung der Dienste zahlen. Dies würde wiederum den Gewinn schmälern. Es lohnt sich am Ende nicht, den Preis für Dienste $(p)$ zu senken, da die Zahlungen an den Konkurrenten für den Netzzugang $(a)$ in der Summe zunehmen würden. Die Folge daraus ist, daß ein hohes Zusammenschaltungsentgelt, das pro durchgeleitete Diensteinheit gezahlt

lich anfallenden Kosten, die durch die Grenzkosten oder durch die inkrementellen Kosten für die Zusammenschaltung berücksichtigt werden, ebenso die Opportunitätskosten beinhalten. Vgl. hierzu Kap. 5.1.6 und 5.2.4.

Vgl. u.a. Laffont/Tirole (2000), S. 190. 
wird, den Wettbewerb im nachgelagerten Markt abschwächt, d.h. andere Wettbewerber, die sowohl in den vor- als auch in den nachgelagerten Markt eintreten wollen, am Markteintritt gehindert werden. Bei einem hohen Zusammenschaltungsentgelt ist der Punkt schnell erreicht, an dem es sich für einen Betreiber nicht mehr lohnt, den Preis im nachgelagerten Markt zu senken. Dieser Effekt der Kostenspirale liefert die Argumentationsgrundlage für kollusives Verhalten bei einer Verhandlungslösung in bezug auf das Zusammenschaltungsentgelt und wirkt wie eine strategische Markteintrittsbarriere. Die Konsumenten sind die eigentlichen Betroffenen, weil sich ein hohes Zusammenschaltungsentgelt am Ende im Endproduktpreis für den erhaltenen Dienst niederschlägt, den sie letztendlich für die Inanspruchnahme eines Dienstes zu zahlen haben.

Da hier davon ausgegangen wird, daß die Konsumenten alle bereitgestellten Dienste unterschiedlicher Anbieter als perfekte Substitute wahrnehmen, bleibt unberücksichtigt, daß Kunden die angebotenen Dienste der Betreiber gar nicht als homogene Produkte empfinden. Die Annahme des Marktaustritts schon bei einem marginal höheren Preis als der des Konkurrenten ist dementsprechend bei Aufgabe der Annahme über die Homogenität der Produkte nicht mehr haltbar. Im folgenden Kapitel soll untersucht werden, inwiefern sich die Berücksichtigung einer begrenzten Substitutierbarkeit der Teilnehmeranschlüsse der verschiedenen Betreiber auf kollusives Verhalten auswirkt.

\subsubsection{Bertrand- WeTtBeWerb Bei horizontaler ProdukT- DIFFERENZIERUNG}

Es soll wieder von zwei Netzbetreibern ausgegangen werden, die beide ähnliche Kostenstrukturen aufweisen und über einen gleich großen Teilnehmeranschlußbereich verfügen. D.h. jeder Anbieter terminiert jeweils die Hälfte aller Telekommunikationsdienste. Außerdem soll explizit auf die Produktdifferenzierung mit Hilfe des Hotelling- Modells eingegangen werden. Die Berllcksichtigung von Produktdifferenzierung seitens der Nachfrager rechtfertigt preisunelastisches Verhalten. Daraus folgt, daß es bei Preisdifferenzen zu keinem sofortigen Marktaustritt kommt, so wie in Kapitel 5.3.1 noch angenommen worden ist. Im Folgenden soll das Verhalten der Anbieter auf dem nachgelagerten Markt anhand simultanen nicht-kooperativen Preiswettbewerbs bei beschränkter Substituierbarkeit analysiert werden. Das Zusammenschaltunsgentgelt wird beim Preiswettbewerb auf dem Endproduktmarkt als gegeben hingenommen. Es ist zuvor von den Anbietern kooperativ gewählt worden. 


\subsubsection{MOdELLIERUNG DER MARKTANTEILE}

Unter der Annahme, daß es zwei Anbieter gibt, die vollständig den gesamten Markt abdecken, werden $\alpha$ Dienste beim Betreiber 1 und $(1-\alpha)$ Dienste beim Anbieter 2 terminiert, d.h. $0<\alpha<1$. Der Marktanteil des jeweiligen Betreibers ist sowohl von seinem eigenen Endproduktpreis als auch von dem des Konkurrenten abhängig: $\alpha=\alpha\left(p_{1}, p_{2}\right)$. Verfügen beide Anbieter über gleich große Marktanteile, dann gilt: $\alpha=(1-\alpha)=1 / 2$.

In Abbildung 5.3-2 ist die Aufteilung der Telefondienste, die innerhalb des eigenen Netzes terminiert werden und die im Netz des anderen beendet werden, nachvollzogen worden. Dabei steht $O_{1}$ für die Originierung und $T_{1}$ für die Terminierung im Netz 1 und dementsprechend $O_{2}$ für die Originierung und $T_{2}$ für die Terminierung im Netz 2. Ein Dienst, der in demselben Netz originiert und terminiert wird, ist folglich ein On-net- Dienst. Ein Gespräch, das in einem anderen Netz terminiert als es originiert wurde, entspricht einem Off-net- Dienst.

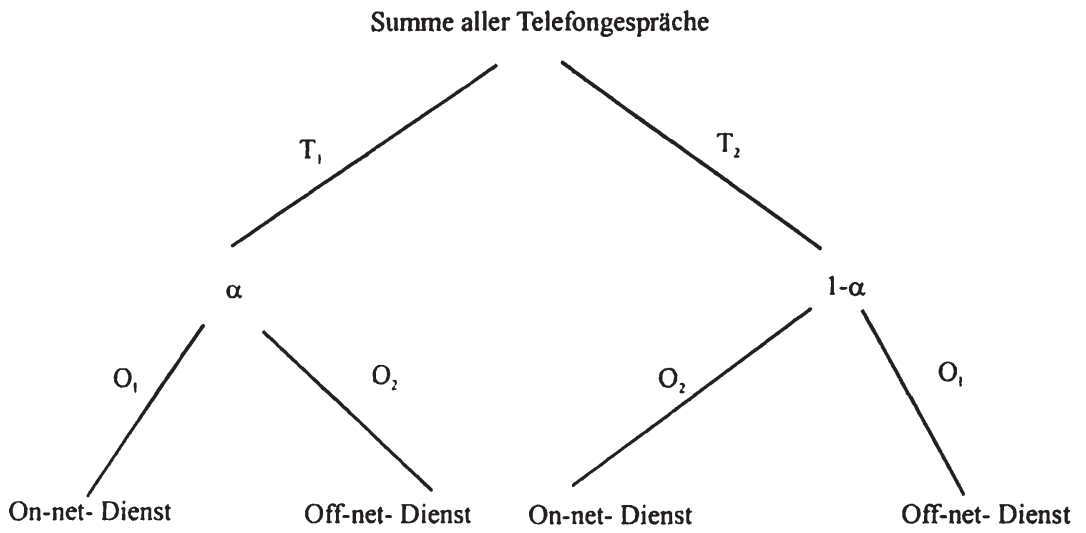

\section{Abbildung 5.3-2: Aufteilung der Telefongespräche in On-net- und Off-net- Dienste}

In der folgenden Analyse haben die Betroffenen bereits ein reziprokes Zugangsentgelt kooperativ gewählt, d.h. $a_{1}=a_{2}=a .^{95}$ Mit anderen Worten zahlt jeder Betreiber pro durchgeleitete Einheit dem anderen dasselbe Zusammenschaltungs-

95 In Deutschland ist es gesetzlich vorgegeben, daß ein Zusammenschaltungsentgelt einheitlich sein soll. Vgl. dazu Kap. 2.4.3. 
entgelt. ${ }^{96}$ Es ergibt sich ein Gesamtgewinn aus On-net- und Off-net- Diensten für den Netzbetreiber 1 :

$$
\Pi_{1}=\alpha x_{1}\left(p_{1}\right)\left[p_{1}-c_{0}-c_{1}\right]+\alpha(1-\alpha)\left[x_{2}\left(p_{2}\right) a_{1}-x_{1}\left(p_{1}\right)\left(c_{0}+a_{1}\right)\right]-k_{1} \text {. }
$$

$k_{1}$ wird im Folgenden außer Acht gelassen, da es bei Grenzwertbetrachtungen ohnehin entfällt.

Der Gesamtgewinn des Carriers 1 ergibt sich aus drei Komponenten:

- Erstens aus den Erlösen und Kosten bedingt durch die Terminierung sämtlicher Dienste On-net wie Off-net:

$$
\pi_{1}^{T}=\propto x_{1}\left(p_{1}\right)\left[p_{1}-c_{0}-c_{1}\right] .
$$

- Zweitens aus den Einnahmen der ,Off-net calls', die im Netz 2 originiert werden und die dann aber an einen Gesprächspartner in das Netz des Betreibers 1 weitergeleitet werden:

$$
\alpha(1-\alpha)\left[x_{2}\left(p_{2}\right) a_{1}\right]
$$

- Drittens aus den Kosten für die Zusammenschaltung von ,Off-net calls', die wiederum in Netz 1 originiert werden und dann aber in das andere Netz weitergeleitet werden:

$$
\alpha(1-\alpha) x_{1}\left(p_{1}\right)\left(c_{0}+a_{1}\right)<0 .
$$

Um die Terminologie zu vereinfachen, soll im weiteren gelten:

$$
\begin{aligned}
& A_{1}=\pi_{1}^{T}, \\
& G_{1}=\alpha(1-\alpha) x_{2}\left(p_{2}\right), \\
& D_{1}=\alpha(1-\alpha) x_{1}\left(p_{1}\right)<0 .
\end{aligned}
$$

Dann lautet der Gesamtgewinn für den Carrier 1:

$$
\Pi_{1}=A_{1}+G_{1} a_{1}-D_{1}\left(c_{0}+a_{1}\right) \text {. }
$$

Ebenso läßt sich der Gewinn des zweiten Anbieters bestimmen.

$$
\Pi_{2}=(1-\alpha) x_{2}\left(p_{2}\right)\left[p_{2}-c_{0}-c_{2}\right]+\alpha(1-\alpha)\left[x_{1}\left(p_{1}\right) a_{2}-x_{2}\left(p_{2}\right)\left(c_{0}+a_{2}\right)\right]
$$

bzw.

$$
\Pi_{2}=A_{2}+G_{2} a_{2}-D_{2}\left(c_{0}+a_{2}\right) .
$$

Bei Ermittlung des Gewinnmaximums soll gelten:

96 Im symmetrischen Fall ist ein einheitlicher Tarif durchaus legitimierbar. Im asymmetrischen Fall dagegen, kann es interessant sein, individuelle Zusammenschaltunsgsentgelte abzuleiten. $\mathrm{Da}$ bei unterschiedlichem Marktanteil und dementsprechend unterschiedlicher Netzwerkgröße die Anforderungen der betroffenen Betreiber auch unterschiedlich sind, werden dementsprechend die optimalen Zusammenschaltunsgsentgelte voneinander abweichen. 
(5.3.9)

$$
\begin{aligned}
& \frac{\partial \Pi_{1}}{\partial p_{1}}=0 \\
& \frac{\partial \Pi_{2}}{\partial p_{2}}=0
\end{aligned}
$$

Die Maximierung von (5.3.6) und (5.3.8) ergeben:

$$
\begin{aligned}
& \frac{\partial \Pi_{1}}{\partial p_{1}}=\frac{\partial A_{1}}{\partial p_{1}}+\frac{\partial G_{1}}{\partial p_{1}} a_{1}-\frac{\partial D_{1}}{\partial p_{1}}\left(c_{0}+a_{1}\right)=0 \\
& \frac{\partial \Pi_{2}}{\partial p_{2}}=\frac{\partial A_{2}}{\partial p_{2}}+\frac{\partial G_{2}}{\partial p_{2}} a_{2}-\frac{\partial D_{2}}{\partial p_{2}}\left(c_{0}+a_{2}\right)=0 .
\end{aligned}
$$

Beide Gleichungen (6.3.6 ) und (6.3.8 ) können in eine Matrix umformuliert werden:

$$
\left(\begin{array}{cc}
\frac{\partial G_{1}}{\partial p_{1}} & -\frac{\partial D_{2}}{\partial p_{2}} \\
-\frac{\partial D_{1}}{\partial p_{1}} & \frac{\partial G_{2}}{\partial p_{2}}
\end{array}\right) \quad\left(\begin{array}{l}
a_{1} \\
a_{2}
\end{array}\right)=\left(\begin{array}{c}
-\frac{\partial A_{1}}{\partial p_{1}}+\frac{\partial D_{1}}{\partial p_{1}} c_{0} \\
-\frac{\partial A_{2}}{\partial p_{2}}+\frac{\partial D_{2}}{\partial p_{2}} c_{0}
\end{array}\right)
$$

Mit Hilfe der Matrix kann die Hauptdeterminante ermittelt werden:

$$
|\Delta|=\left|\begin{array}{cc}
\frac{\partial G_{1}}{\partial p_{1}} & -\frac{\partial D_{2}}{\partial p_{2}} \\
-\frac{\partial D_{1}}{\partial p_{1}} & \frac{\partial G_{2}}{\partial p_{2}}
\end{array}\right|=\frac{\partial G_{1}}{\partial p_{1}} * \frac{\partial G_{2}}{\partial p_{2}}-\left[-\frac{\partial D_{1}}{\partial p_{1}} * \frac{\partial D_{2}}{\partial p_{2}}\right] \neq 0 .
$$

Da $|\Delta| \neq 0$ ist, sind $\Pi_{1}$ und $\Pi_{2}$ voneinander linear und funktional unabhängige Funktionen. ${ }^{97}$ Damit ist eine Komponente bestimmt, die die Möglichkeit kollusiven Verhaltens zwischen den beiden Anbietern erläutert, da $a \neq 0$ ist. Obwohl die Endproduktpreise $p_{1}, p_{2}$ seitens der Unternehmen nicht kooperativ bestimmt werden, können dennoch jedes beliebig gewünschte Preisgleichgewicht $p_{1}^{*}, p_{2}^{*}$ durch eine angemessene Wahl an Zusammenschaltungsgentgelten erzielt werden. $^{98}$ 


\subsubsection{HORIZONTAle Produktdifferenzierung}

Hier soll explizit darauf eingegangen werden, daß verschiedene Anbieter keine homogenen Produkte bereitstellen. Beispielsweise wird ein Fernsehkabelbetreiber neben dem Einspeisen von Fernsehprogrammen zusätzlich Telekommunikationsdienste anbieten. Er wird versuchen, die Produkte kombiniert bereitzustellen. Ein Stromanbieter wird ebenso Telefondienste zusammen mit Stromdiensten bereitstellen. Kombinationsmöglichkeiten können sich sowohl inhaltlich auf Mehrwertdienste als auch auf Abrechnungen, Wartung, Kundendienst beziehen. Das kann sich wiederum auf die Produktvielfalt wie auch auf die Vertragsausgestaltung sowie auf die Vertragslaufzeit auswirken. Dabei werden die Anbieter jede Gelegenheit wahrnehmen, Verbundvorteile auszunutzen.

Auf der anderen Seite wird der Kunde die von den unterschiedlichen Betreibern angebotenen Telekommunikationsdienste 'nicht als vollständige Substitute empfinden. ${ }^{99}$ Mit anderen Worten liegt ein hoher Produktdifferenzierungsgrad vor, und die Nachfrager reagieren relativ unelastisch auf Preisveränderungen, d.h., daß Kunden bei Preisunterschieden nicht sofort auf einen anderen Betreiber ausweichen können und wollen. Dies impliziert, daß Preisdifferenzen im Endproduktpreis zwischen den beiden Anbietern nicht zwangsläufig zu einer Marktverdrängung des teureren Anbieters fuhren müssen. Der Trade-off besteht entweder zu einem hohen Preis nur einen Teil der Nachfrage zu bedienen oder $\mathrm{zu}$ einem niedrigen Preis den gesamten Markt abzudecken. Im Unterschied zum klassischen Bertrand- Fall, bei dem der Anbieter bei einem höheren Preis als der des Konkurrenten vollständig vom Markt verdrängt wird, gibt es hier die Möglichkeit, trotz eines höheren Preises am Markt präsent zu bleiben, selbst wenn nur eine geringe Menge abgesetzt wird. Der Anreiz, das andere Unternehmen zu unterbieten, um einen größeren Marktanteil abzudecken, wird durch den einhergehenden Preisverfall gedämpft.

Das klassische Bertrand- Gleichgewicht, wie es in Kapitel 5.3.1 hergeleitet wurde, unterstellt die Existenz eines globalen Maximums. Wenn aber Produktdifferenzierung in der Analyse zugelassen wird, dann ist das lokale Maximum kein globales Maximum mehr.

99 Bestehen von Switching- Costs aufgrund von Markenloyalităt bzw. Kundentreue oder Netzwerkgröße wird hier nicht betrachtet, obwohl es ein wichtiger Gesichtspunkt ist. Ihre Berücksichtigung ändert allerdings nichts an der Richtung des Ergebnisses. Carter/Wright (1996, 1999) fugen eine zusătzliche Variable in die Nutzenfunktion ein, die die Markenloyalität der Konsumenten für einen Betreiber widerspiegelt, berücksichtigen aber ebenso nicht die Switching- Costs. 
Zwecks Modellierung der Nachfrageseite sind die Konsumentenpräferenzen separierbar und durch eine quasi-lineare Nutzenfunktion darstellbar: ${ }^{100}$

$$
U=u\left(x_{B}\right)-\Theta+y
$$

$u\left(x_{B}\right)$ repräsentiert den Nutzen, den ein Konsument aus der Netzmitgliedschaft verspürt. Der Grenznutzen kann mit zunehmender Zahl an Telefongesprächen zunehmen. Die nähere Ausgestaltung findet sich in Kapitel 4.2. ${ }^{101}$

$Y$ soll für den Konsum aller anderen Güter stehen.

Mit $\Theta$ wird in der Nutzenfunktion die Bedeutung der Substituierbarkeit der angebotenen Dienste für die Konsumentenpräferenzen berücksichtigt. Hierzu wird das Hotelling- Modell zur Hilfe genommen. ${ }^{102}$

Das Hotelling- Modell ${ }^{103}$ modelliert den Substitutionsgrad zwischen den von den beiden Betreibern angebotenen Produkten. Die Heterogenität der Produkte wird räumlich definiert: Es wird ein eindimensionaler Eigenschafts-Produktraum $\mathrm{z}$ definiert, in der die Verteilung der Produkteigenschaften wiedergegeben wird. Es wird dabei von einer uniformen Verteilung ausgegangen: $z \in[0,1]$. Die beiden Produkte der jeweiligen Betreiber liegen jeweils an den Enden des Intervalls ( $z_{1}=0$ und $z_{2}=1$ ). Bei der Wahl des Konsumenten für einen Betreiber hat der Konsument Nutzeneinbußen $(w)$ hinzunehmen, weil er ein Gut in der von ihm präferierten Eigenschaft nicht kaufen kann. $\Theta$ soll in diesem Zusammenhang die Kosten umfassen, die entstehen, wenn ein Sender eines Datenstroms mit dem anderen Netz $z_{B}$ (wobei $B=1,2$ ) verbunden wird: $\Theta=w\left|\bar{z}-z_{B}\right| \cdot{ }^{104}$ Es gilt, daß $\Theta$ $<0$ ist. Der Konsument, der sich im Raum an Stelle $\widetilde{z}$ befindet, hat $w \widetilde{z}$ Nutzeneinbußen bei der Entscheidung Teilnehmer des Netzwerks 1 zu werden und $w(1-\widetilde{z})$, um Teilnehmer von Netzwerk 2 zu werden. D.h. der Konsument an $\widetilde{z}$ ist gerade indifferent in seiner Wahl zwischen beiden Netzbetreibern.

Die indirekte Nutzenfunktion wird mittels Maximierung der Nutzenfunktion abzüglich einer allgemein formulierten Budgetrestriktion ermittelt. ${ }^{105}$

\footnotetext{
100 Vgl. auch Carter/Wright (1999), S. 10.

101 Laffont u.a. (1998), S. 7 modellieren eine Nutzenfunktion in Abhängigkeit von der Nachfrageelastizität. In Carter/Wright (1999), S. 10 wird eine allgemeine lineare Funktion angenommen.

Für die Modellierung der Nachfrage wurde in der Regel auf den Hotelling-Ansatz zurückgegriffen. Vgl. Armstrong (1998); Carter/Wright (1999); Laffont u.a. (1998). Doganoglu/ Taumann (1998) dagegen benutzen einen, random utility model approach". Vgl. Hotelling (1929); Pfähler/Wiese (1991) S. 461; Tirole (1995), S. 213 ff.

104 Vgl. Laffont u.a. (1998), S. 6.

Ins Die über die Maximierung der Nutzenfunktion abzüglich der Budgetrestriktion ermittelte Nachfragefunktion wird in die Nutzenfunktion wieder eingesetzt. Auf diese Weise kann eine indirekte Nutzenfunktion ermittelt werden. Folgende Herleitung der Marktanteile vgl. Laffont u.a. (1998), S. 7.
} 


$$
v\left(p_{B}\right)=\max _{x_{B}}\left\{u\left(x_{B}\right)-p_{B} x_{B}\right\} .
$$

Ist der Konsument indifferent in seinem Beitritt in das eine oder das andere Netzwerk $(\widetilde{z})$, dann entspricht sein Nettonutzen aus der Teilnahme an Netzwerk 1 dem Nettonutzen aus der Teilnahme an Netzwerk 2:

$$
v\left(p_{1}\right)-w \widetilde{z}=v\left(p_{2}\right)-w(1-\widetilde{z}) .
$$

Aus (5.3.13) folgt, daß die Nutzeneinbuße, die dem Konsumenten bei der Entscheidung furr das eine oder andere Netzwerk entsteht, gleich groß ist.

Es gilt: $\alpha_{B}=z_{B}$. Für die Höhe des Marktanteils des Betreibers 1 lautet dann das Kalkül:

$$
\alpha_{B}\left(p_{1}, p_{2}\right)=\left\{\begin{array}{lll}
0 & \text { für } & v\left(p_{1}\right)-w \tilde{z}<v\left(p_{2}\right)-w(1-\tilde{z}) \\
1 & \text { für } & v\left(p_{1}\right)-w \widetilde{z}>v\left(p_{2}\right)-w(1-\tilde{z}) \\
\widetilde{z} & \text { für } \quad v\left(p_{1}\right)-w \tilde{z}=v\left(p_{2}\right)-w(1-\tilde{z})
\end{array}\right.
$$

Wenn $\alpha=\widetilde{z}$ ist, dann läßt sich Gleichung (5.3.13) ebenso schreiben:

$$
v\left(p_{1}\right)-w \alpha=v\left(p_{2}\right)-w(1-\alpha) .
$$

Dies nach $\alpha$ aufgelöst ergibt:

$$
\alpha=\frac{1}{2}+\frac{1}{2 w}\left[v\left(p_{1}\right)-v\left(p_{2}\right)\right]
$$

wobei gilt: $\quad \frac{1}{2 w}=\sigma$.

$\sigma$ stellt den Grad an Produktdifferenzierung bzw. Substituierbarkeit zwischen den beiden Netzwerken seitens der Konsumenten dar. Ist die Nutzeneinbuße, die bei der Wahl für das eine oder andere Netzwerk entsteht, sehr gering $(w \rightarrow 0)$, dann ist $\sigma$ besonders groß. Beide Netzwerke werden als recht unterschiedliche Produkte seitens der Konsumenten empfunden, die sich schlecht substituieren lassen. Daraus leitet sich fur die Anbieter eine gewisse Flexibilität in bezug auf Preisdifferenzen ab. Ein Anbieter kann deutlich von dem Endproduktpreis des Konkurrenten nach oben hin abweichen, ohne befürchten zu müssen, daß seine Kunden zu dem anderen Netzbetreiber wechseln.

Ist die Nutzeneinbuße relativ hoch $(w \rightarrow \infty)$, dann geht $\sigma \rightarrow 0$, d.h. die Produkte werden zunehmend als homogen empfunden. Preisdifferenzen werden sich schnell auf die Höhe des jeweiligen Marktanteils auswirken. 


\subsubsection{VERHANDLUNGSLÖSUNG}

Die Annahme symmetrischer Marktbedingungen impliziert folgende Vereinfachungen: Aus der Annahme, da $\beta \alpha=(1-\alpha)=1 / 2$, ergibt sich da $\beta$

$$
\frac{\partial \alpha(1-\alpha)}{\partial p_{i}}=0 \text {. }
$$

Daraus folgt wiederum, daß $\frac{\partial G_{i}}{p_{i}}=0$ und daß $\frac{\partial D_{i}}{\partial p_{i}}=\alpha(1-\alpha) \frac{\partial x_{i}}{\partial p_{i}}$.

Dann läßt sich folgende Hauptdeterminante unter der Annahme von Symmetrie bestimmen: ${ }^{106}$

$$
\begin{aligned}
& |\Delta|=\left[\alpha(1-\alpha) \frac{\partial x_{1}}{\partial p_{1}} * \alpha(1-\alpha) \frac{\partial x_{2}}{\partial p_{2}}\right] \\
& |\Delta|=\alpha^{2}(1-\alpha)^{2} \frac{\partial x_{1}}{\partial p_{1}} * \frac{\partial x_{2}}{\partial p_{2}} \quad{ }^{107}
\end{aligned}
$$

$\mathrm{Da}|\Delta| \neq 0$ ist, ist eine notwendige Bedingung gegeben, daß $\mathrm{a} \neq 0$ ist. D.h. es läßt sich bereits wie schon in Gleichung (5.3.11) dargestellt an der Hauptdeterminante ein Anreiz zu kollusivem Verhalten erkennen.

Bestimmung der Unterdeterminanten:

$$
\begin{aligned}
& \left|\Delta_{1}\right|=\left(\frac{\partial A_{2}}{\partial p_{2}}-\frac{\partial D_{2}}{\partial p_{2}} c_{0}\right) * \frac{\partial D_{2}}{\partial p_{2}} \\
& \left|\Delta_{2}\right|=\frac{\partial D_{1}}{\partial p_{1}} *\left(\frac{\partial A_{1}}{\partial p_{1}}-\frac{\partial D_{1}}{\partial p_{1}} c_{0}\right) .
\end{aligned}
$$

Aus (5.3.18) und (5.3.19) ergeben sich bei symmetrischen Marktbedingungen:

$$
\begin{aligned}
& \left|\Delta_{1}\right|=\left[\frac{\partial(1-\alpha)}{\partial p_{2}} x_{2}\left(p_{2}-c_{0}-c_{2}\right)+(1-\alpha) \frac{\partial x_{2}}{\partial p_{2}}\left(p_{2}-c_{0}-c_{2}\right)-\alpha(1-\alpha) \frac{\partial x_{2}}{\partial p_{2}} c_{0}\right] \alpha(1-\alpha) \frac{\partial x_{2}}{\partial p_{2}} \\
& \left|\Delta_{2}\right|=\left[\frac{\partial \alpha}{\partial p_{1}} x_{1}\left(p_{1}-c_{0}-c_{1}\right)+\alpha \frac{\partial x_{1}}{\partial p_{1}}\left(p_{1}-c_{0}-c_{1}\right)-\alpha(1-\alpha) \frac{\partial x_{1}}{\partial p_{1}} c_{0}\right] \alpha(1-\alpha) \frac{\partial x_{1}}{\partial p_{1}}
\end{aligned}
$$

$106 \mathrm{Vgl}$. zur Bestimmung von Haupt- und Unterdeterminante Anhang A.2.

107 Vgl. Carter/Wright (1996), S. 20. 
Dadurch, daß $\left|\Delta_{1}\right| \neq 0$ und $\left|\Delta_{2}\right| \neq 0$ ist, ist auch die hinreichende Bedingung gegeben, daß Anbieter 1 und Anbieter 2 ein Zusammenschaltungsentgelt, das ungleich Null ist, als optimal empfinden. Aus (5.3.16) folgt

$$
\begin{aligned}
& \alpha\left(p_{1}, p_{2}\right)=\frac{1}{2}+\sigma\left[u\left(x_{1}\right)-p_{1} x_{1}-\left[u\left(x_{2}\right)-p_{2} x_{2}\right]\right. \\
& \frac{\partial \alpha}{\partial p_{1}}=-\sigma x_{1} \\
& \frac{\partial \alpha}{\partial p_{2}}=\sigma x_{2},
\end{aligned}
$$

so daß für die Bestimmung der Unterdeterminanten gilt:

$$
\begin{aligned}
& \left|\Delta_{1}\right|=\left[\sigma x_{2}^{2}\left(p_{2}-c_{0}-c_{2}\right)+(1-\alpha) \frac{\partial x_{2}}{\partial p_{2}}\left(p_{2}-c_{0}-c_{2}\right)-\alpha(1-\alpha) \frac{\partial x_{2}}{\partial p_{2}} c_{0}\right] \alpha(1-\alpha) \frac{\partial x_{2}}{\partial p_{2}} \\
& \left|\Delta_{2}\right|=\left[-\sigma x_{1}^{2}\left(p_{1}-c_{0}-c_{1}\right)+\alpha \frac{\partial x_{1}}{\partial p_{1}}\left(p_{1}-c_{0}-c_{1}\right)-\alpha(1-\alpha) \frac{\partial x_{1}}{\partial p_{1}} c_{0}\right] \alpha(1-\alpha) \frac{\partial x_{1}}{\partial p_{1}} .
\end{aligned}
$$

Mit der Einfuhrung des Produktdifferenzierungsgrades lassen sich Zusammenhänge zwischen den Substitutionsmöglichkeiten zwischen den beiden Betreibern und der Anreiz zu kollusivem Verhalten herstellen.

Unter folgenden vereinfachenden Annahmen

- den Anbietern steht eine einheitliche Nachfrage gegenüber:

$$
x_{1}\left(p_{1}\right)=x_{2}\left(p_{2}\right)=x(p) \text {, }
$$

- es handelt sich um identische Unternehmen : $c_{1}=c_{2}=c$,

- es ist gesetzlich ein einheitliches Zusammenschaltungsentgelt vorgegeben: $a_{1}=a_{2}=a$,

kann sich ein optimales Zusammenschaltungsentgelt für beide Verhandlungspartner bei nicht-kooperativ gewăhlten Endproduktpreisen ergeben.

Mit Hilfe von Haupt- und Unterdeterminate kann das gegebene reziproke $\mathrm{Zu}$ sammenschaltungsentgelt formuliert werden:

$$
a=\frac{\left|\Delta_{B}\right|}{|\Delta|} .
$$

Dann ergibt sich folgendes Zusammenschaltungsentgelt: 


$$
a=\frac{\left[\sigma x^{2}\left(p-c_{0}-c\right)+(1-\alpha) \frac{\partial x}{\partial p}\left(p-c_{0}-c\right)-\alpha(1-\alpha) \frac{\partial x}{\partial p} c_{0}\right] \alpha(1-\alpha) \frac{\partial x}{\partial p}}{\alpha^{2}(1-\alpha)^{2}\left(\frac{\partial x}{\partial p}\right)^{2}}
$$

bzw. für $\gamma=x^{2}\left(p-c_{0}-c\right)+(1-\alpha) \frac{\partial x}{\partial p}\left(p-c_{0}-c\right)-\alpha(1-\alpha) \frac{\partial x}{\partial p} c_{0}$

$$
a=\frac{\sigma \gamma}{\alpha(1-\alpha) \frac{\partial x}{\partial p}} .
$$

Die Höhe des Zusammenschaltungsentgelts ist von dem Produktdifferenzierungsgrad der angebotenen Dienste abhängig. Ein hoher Produktdifferenzierungsgrad $(\sigma \rightarrow \infty)$ verstärkt den Anreiz, sich auf ein hohes Zusammenschaltungsentgelt zu einigen. Das Zusammenschaltungsentgelt wird letztendlich unter diesen Bedingungen so ansteigen, bis der Endproduktpreis für Dienste einem Preis entspricht, der sich in einer Monopolsituation herausbilden würde. Beide Anbieter können sich nur unter der Annahme, daß sich ihre Dienste gar nicht substituieren lassen, wie Monopole auf dem Markt fur Telekommunikationsdienste benehmen. ${ }^{108}$ Der Anreiz zu Kollusion ist, wie schon in Kapitel 5.3.1 formuliert, aufgrund des Kostenspiraleneffektes gegeben. Ein hoher Produktdifferenzierungsgrad führt dazu, daß das sich unter Kollusion ergebende Gleichgewicht beständig ist. Die Firmen haben keinen Anreiz, von dem kollusiven Endproduktpreis abzuweichen, wenn die bereitgestellten Dienste nicht als Substitute empfunden werden. Unter diesen Umständen läßt sich die Frage erörtern, ob regulierende Eingriffe notwendig sind. ${ }^{109}$

Ein sehr geringer Produktdifferenzierungsgrad $(\sigma \rightarrow 0)$ kann dementsprechend ein Sinken des Zusammenschaltungsentgelts bewirken. Je stärker sich nämlich die Endprodukte der Anbieter substituieren lassen, desto eher ist Kollusion nicht mehr stabil. Die Anreize zu kollusivem Verhalten in bezug auf die Verhandlung des Zusammenschaltungsentgelts bleiben allerdings nach wie vor bestehen. Ein $\mathrm{Zu}$ sammenschaltungsentgelt in Höhe der anfallenden relevanten Kosten wird aufgrund des Kostenspiraleneffektes kein stabiles Ergebnis sein. Ein geringer Poduktdifferenzierungsgrad wird den Effekt der Kostenspirale nicht kompensieren können. Monopolpreise im Endproduktmarkt werden allerdings wegen der Be-

\footnotetext{
108 Beweis vgl. im Anhang A.3. Dieses Ergebnis entspricht dem Ergebnis der Baumol-WilligRegel bei nicht-reguliertem Endproduktmarkt. Vgl. Baumol (1999).

Vgl. dazu Kap. 5.3.2.4.
} 
rücksichtigung eines geringen Produktdifferenzierungsgrades ebenso nicht stabil sein. ${ }^{110}$ Das Entgelt für die Zusammenschaltung wird bei nicht-kooperativ gewählten Endproduktpreisen irgendwo zwischen Grenzkostenpreisen und Monopolpreisen liegen.

Als Fazit läßt sich festhalten, daß bei starken Substituten kollusives Verhalten in bezug auf die Bestimmung des Zusammenschaltungsentgelts nicht stabil ist. Es ergibt sich keine Notwendigkeit, regulierende Eingriffe zwingend zu tätigen.

\subsubsection{WIRTSCHAFTSPOLITISCHE EMPFEHLUNG}

Aus der Tatsache, daß grundsătzlich Anreize zu Kollusion bei der Verhandlung von Zusammenschaltungsentgelten bestehen, kann nicht eine prinzipielle Notwendigkeit regulierender Eingriffe abgeleitet werden:

1) Die Höhe des Produktdifferenzierungsgrades ist entscheidend, ob das unter Kollusion gewăhlte Gleichgewicht überhaupt stabil ist.

Aus der Analyse des vorangegangenen Kapitels hat sich ergeben, daß die Stabilităt kollusiven Verhaltens vom Substitutionsgrad abhängt. Bei hohem Substitutionsgrad ist nicht mehr davon auszugehen, daß Kollusion stabil ist. Unter diesen Umständen ist eine Regulierung der Zusammenschaltungsentgelte nicht erforderlich.

2) Kollusionsanreize können auf die zugrunde liegende Annahmestruktur des hier vorgestellten Modells zurückzuführen sein. In der Realität liegen viel kompliziertere Preis- und Tarifgestaltungen vor, als hier untersucht worden ist.

Beispielsweise ist in dem Modell unterstellt worden, daß lineare Tarife vorliegen. Bei Vorliegen nicht-linearer Preise wie z.B. bei 2-Part-Tarifen im Endproduktmarkt (monatliche Grundgebuhr plus Preis pro telefonierte Einheit) muß das Zusammenschaltungsentgelt nicht zwangsläufig als Kollusionsinstrument mißbraucht werden. Je höher der Gewinn pro Kunde ist, desto härter ist der Wettbewerb um Marktanteile. Dann kann die fixe Gebühr zum Kernbestandteil des Wettbewerbs werden. ${ }^{\prime \prime}$ Laffont/Tirole (2000, S. 200) dagegen zeigen, daß der optimale 2-Part-Tarif so gestaltet sein kann, daß die Preise pro Einheit den anfallenden Grenzkosten entsprechen. Dann sind die Gewinne pro Gespräch gleich Null. Der Betreiber macht insgesamt nur dann einen Gewinn, wenn die monatliche Grundgebühr größer ist als die anfallenden Fixkosten pro Kunde. D.h. das Zusammenschaltungsentgelt ist nicht länger mehr ein Kollusionsinstrument. Es läßt

110 Es ist nicht eindeutig bestimmbar, wo ein stabiles Gleichgewicht liegt, außer das es zwischen Monopolpreis und Grenzkosten liegen muß.

111 So werden im Mobilfunk inzwischen schon Handys für 1,- DM angeboten. Vgl. auch Armstrong (1997). 
sich schlußfolgern, daß Kollusion womöglich nur unter der Annahme linearer Preise gilt. Bei zwei geteilten Tarifen ist Kollusion nicht mehr stabil. ${ }^{112}$

Weiterhin wurde angenommen, daß keine Preisdiskriminierung vorliegt. Wird Preisdiskriminierung in der Analyse zugelassen und ist das Zusammenschaltungsentgelt höher als die anfallenden Grenzkosten für die Gewährung von Netzzugang, dann sind Telefondienste, die in dem Netz des Konkurrenten terminiert werden, teurer als Dienste, die innerhalb des Netzes terminiert werden. Ein Anbieter wird einen Anreiz haben, aufgrund der Existenz tarifbedingter Netzexternalitäten niedrigere Endproduktpreise für On-net- Dienste zu nehmen. Ein Betreiber kann seinen Marktanteil erhöhen, indem er seine On-net Preise senkt. Daraus folgt, daß hohe Zusammenschaltungsentgelte nicht zwangsläufig zu hohen Endproduktpreisen führen müssen. ${ }^{113}$

Außerdem wurden Seitenzahlungen in der Analyse ausgeschlossen. Ist das $\mathrm{Zu}$ sammenschaltungsentgelt größer als die entstandenen Grenzkosten für die Gewährung von Netzzugang, dann besteht der Anreiz für den Anbieter, dem Empfänger eines Telefongesprächs etwas zu zahlen, damit er ein Gespräch aus dem anderen Netz entgegennimmt. ${ }^{14}$ Auf diese Weise werden Gewinne aus hohen Zugangsentgelten wegkonkurriert. Kollusives Verhalten ist dann nicht mehr stabil.

In einer Situation eines hohen Produktdifferenzierungsgrades, die Monopolpreise auf dem Endproduktmarkt begünstigen, bleiben regulatorische Eingriffe abzuwägen. Dann aber muß eine differenzierte Analyse der Ausgestaltung des regulatorischen Eingriffs erfolgen. Zunächst muß bestimmt werden, welche Parameter reguliert werden sollen. Zum einen kann eine Preisregulierung der Endproduktpreise in Betracht gezogen werden. Carter/Wright $(1996,1999)$ dagegen zeigen, $\mathrm{da} ß$ die Verwendung des Zusammenschaltungsentgelts als Kollusionsinstrument nicht durch Preisregulierung in Griff zu bekommen ist. Regulierung der Endproduktpreise wird zu einer Wahl von $a$ führen, die die gemeinsamen Gewinne maximiert. Das Ergebnis ist wie bei einem Monopol. ${ }^{115}$ Eine Regulierung der End-

112 Dies wiederum gilt womöglich nur bei homogenen Präferenzen. Vgl. Laffont u.a. (1998), S. 20.

113 Vgl. Laffont/Tirole (2000), S. 196 ff. Gans/King (1999) zeigen wiederum auf, daß es bei Vorliegen von nicht-linearen Preisen oder Preisdiskriminierung trotz Bill-and-Keep- Arrangements bezüglich des Zusammenschaltungsentgelts zu Preisabsprachen auf dem Endproduktmarkt kommen kann und Endproduktpreise in die Höhe treibt.

114 Laffont/Tirole sprechen in diesem Zusammenhang von einer Empfanger-Subvention. Vgl. Laffont/Tirole (2000), S. $196 \mathrm{ff}$.

115 Carter/Wright (1996), S. 22; (1998), S. 8 zeigen das für lineare Zusammenschaltungsentgelte. Laffont u.a. (1998), S. 20 zeigen das an 2-Part-Tarifen. Auch Armstrong (1998), S. 557 kommt zu dem Ergebnis, daß das Zusammenschaltungsentgelt höher sein wird, wenn der Endproduktmarkt reguliert ist. 
produktpreise hat keinen Einfluß auf strategisches Verhalten im vorgelagerten Markt. Kollusionsanreize bleiben folglich bestehen.

Verbot kollusiven Verhaltens als regulatorischer Eingriff fuhrt dazu, daß sowohl das Zusammenschaltungsentgelt als auch der Endproduktpreis nicht-kooperativ gesetzt werden. Das Ergebnis ist für die Konsumenten die schlechteste aller Lösungen, weil beide Preise höher ausfallen werden als unter einer Marktlösung. ${ }^{116}$ Die dritte Möglichkeit besteht in der Preisregulierung der Zusammenschaltungsentgelte. Hier wird gegenwärtig sowohl bei der deutschen Regulierungsbehörde als auch bei der Oftel und der FCC das TELRIC- Konzept befurrwortet. Das Problem an diesem Konzept ist, daß die Kostengrößen fulr die einzelnen Netzelemente präzise berechnet werden können müssen. Die bisher in Betracht gezogenen Methoden zur Ermittlung der Kostengrößen (Top-Down- und Bottom-UpAnsatz) sind sehr aufwendig in der Datenermittlung und lösen das Problem nur unbefriedigend. Eine konkrete Analyse über gegenwärtige Konzepte zur Regulierung von Zugangs- und Zusammenschaltungsentgelten wird im folgenden Kapitel 5.4 gegeben.

Zudem sei kritisch angemerkt, daß sich aus dem Anreiz zu kollusiven Absprachen keine Notwendigkeit für die Existenz einer sektorspezifischen Regulierungsbehörde ableiten läßt. Preisliche Absprachen zwischen Wettbewerbern unterliegen in der Regel einem Verstoß gegen allgemeine Wettbewerbsregeln, dessen Kontrolle und Ausführung in den Bereich einer allgemeinen Wettbewerbsbehörde gehört.

\subsection{KOSTENORIENTIERTE RECHNUNGSANSÄTZE IN DER REGULIERUNGSPRAXIS}

Nachdem in den ersten drei Unterkapiteln des Kapitels 5 die Problematik des Zugangs- bzw. Zusamenschaltungsentgelts dargestellt und analysiert worden ist und zwischen theoretischen Lösungen abgewogen worden ist, die das Ziel der ökonomischen Effizienz verfolgen und nicht immer so umsetzbar sind wie gewulnscht, soll in diesem Kapitel sich der Regulierungspraxis gewidmet werden. Grundlage für die praktizierte Regulierung ist in Deutschland das TKG, das sowohl den Zugang als auch die Zusammenschaltung von Telekommunikationsnetzen sowie die

116 In Carter/Wright (1996), S. 25; (1998), S. 8 wird ein Beweis hierfür angeführt. Auch Laffont u.a. (1997), S. 708 weisen darauf hin, daß Betreiber bei nicht-kooperativen Zusammenschaltungsentgelten wegen des, double marginalisation' Problems Anreize haben, das Zusammenschaltungsentgelt in die Höhe zu treiben, bis auf dem Endproduktmarkt Preise in Höhe von Monopolpreisen erzielt werden. Dabei sind die Anreize am größten, wenn die Netzwerke nicht substitutierbar sind. Vgl. auch Buehler (2000). 
Entgeltbestimmung eindeutig regelt. ${ }^{117}$ Von den bestehenden verschiedenen Regulierungsansätzen sollen in diesem Kapitel ausschließlich kostenorientierte Rechnungskonzepte näher vorgestellt werden, da diese im TKG gesetzlich festgehalten sind und von der deutschen Regulierungsbehörde praktiziert werden. ${ }^{118}$

\subsubsection{DAS KOSTENORIENTIERTE REGULIERUNGSVERFAHREN}

Das kostenorientierte Regulierungsverfahren dient dazu, die Kosten für die Bereitstellung schmalbandiger Verbindungsleistungen im Rahmen der Zusammenschaltung von Netzen und der Bereitstellung anderer besonderer Netzzugänge sowie der Überlassung von Teilnehmeranschlußleitungen zu bestimmen. Ein kostenorientierter Regulierungsansatz soll sicherstellen, daß zum einen Newcomer ohne eigene Netzinfrastruktur nicht benachteiligt sind. Zum anderen soll dem Argument der Kostensubadditivität zufolge ein Netzaufbau in Regionen verhindert werden, in denen schon ein Netz besteht und billiger anbietet, als ein neu erbautes könnte. Dabei wird keine Wettbewerbsform bevorzugt.

Im TKG §24 Abs.1 ist das kostenorientierte Regulierungsverfahren vorgegeben. Dort ist festgehalten, daß sich Entgelte an den „Kosten der effizienten Leistungsbereitstellung" orientieren sollen. ${ }^{119}$ Diese Kosten setzen sich nach $\S 3$ Abs. 2 der Telekommunikations-Entgeltregulierungsverordnung (TentgV) aus den langfristigen zusätzlichen Kosten (Inkrementalkosten) der Leistungsbereitstellung zuzüglich eines angemessenen Aufschlages für leistungsmengenneutrale Gemeinkosten zusammen einschließlich einer angemessenen Kapitalverzinsung. Dabei gilt es seitens der Regulierungsbehörde zu prüfen, ob die Entgelte Aufschläge enthalten, die nur aufgrund der marktbeherrschenden Stellung durchsetzbar sind ( $\$ 24$ Abs. 2 und $\$ 27$ Abs. 2,3 TKG). Telekommunikationsunternehmen, die in Deutschland der Entgeltregulierung unterliegen, müssen Kostennachweise vorlegen ( $\$ 2$ TentgV). Die Regulierungsbehörde prüft, ob geltend gemachte Kosten den Kosten der effizienten Leistungsbereitstellung entsprechen. Dabei hat das regulierte Unternehmen der RegTP Nachweise vorzulegen, ob und wie weit sich das beantragte

117 Vgl. hierzu Kap. 2.4.2 sowie 2.4.3.

118 Auch von der amerikanischen Regulierungsbehörde FCC wird das kostenorientierte Rechnungskonzept gesetzlich vorgegeben. Bis Anfang der neunziger Jahre wurde beispielsweise in Großbritannien und den USA allerdings noch das Vollkostenprinzip auf Basis historischer Kosten (,Historical Cost Accounting' - HCA) mittels ,Fully Distributed Cost Pricing' (FDC) praktiziert. Eine weitere Alternative zu den Kostenkonzepten stellt das Vergleichsmarktkonzept dar, das in Deutschland bei den ersten Zusammenschaltungsentscheidungen 1997 Anwendung fand. Vgl. Schütz (2001), S. 552 sowie Kap. 3.

119 Dem Begriff liegt sinngemäß produktive bzw. technische Effizienz zugrunde. Vgl. dazu WIK (1998), S. 4. 
Entgelt an den Kosten der effizienten Leistungsbereitstellung orientiert. Es wird ein Vergleich der nachgewiesenen Ist-Kosten mit den Kosten bei effizienter Leistungsbereitstellung vorgenommen ( $\$ 2$ Abs. 1 und 2 TentgV). Fallen die IstKosten höher aus, sind sie nur bei besonderer Rechtfertigung genehmigungsfähig ( $\$ 3$ Abs. 4 Satz 1 TentgV). ${ }^{120}$

Dem betroffenen Unternehmen wird seitens der EU-Kommission empfohlen, seine Kosten getrennt nach folgenden vier Bereichen zu erfassen: Kosten des Verbindungsnetzes, Kosten des Teilnehmeranschlußnetzes, Kosten fur Dienstleistungen und Kosten sonstiger Tätigkeiten. ${ }^{121}$

Im folgenden soll es um die Entgeltregelung für Zugang und Zusammenschaltung gehen. Die Kostennachweise für das Teilnehmeranschlußnetz werden mit den Ergebnissen von den Berechnungen eines generellen Referenznetzes auf allgemeiner Basis verglichen. Entgeltgenehmigungen werden nur mit Wirkung auf die Zukunft erteilt. Rückwirkende Genehmigungen sind nicht zulässig. Sämtliche Entgeltanträge sowie genehmigte Entgelte werden im Amtsblatt der Regulierungsbehörde veröffentlicht ( $\$ 8$ Abs. 2 TentgV).

Der kostenorientierte Ansatz zielt auf eine Analyse von Kosten ab, die sich erst bei Markteintritt, also in der Zukunft ergeben werden. Grundlage hierfür stellen die zukunftsgerichteten langfristigen Inkrementalkosten dar, die auch als ,Forward Looking Long Run Incremental Costs', kurz FL-LRIC, oder auch nur ,Long Run Incremental Costs' bzw. LRIC bezeichnet werden. ${ }^{122}$ Die langfristigen zusätzlichen Kosten stellen den zurechenbaren Teil der Kosten der effizienten Leistungsbereitstellung dar. Die juristische Definition der Langfristigkeit impliziert, daß bei dem betrachteten zusätzlichen Element bzw. Dienst, das zusätzlich angeboten würde, alle Inputs als variabel angenommen würden, um ökonomische Effizienz gewährleisten zu können. ${ }^{123}$ Die Idee der langfristigen Inkrementalkosten besteht darin, Zugangspreise auf der Basis fiktiver effizienter Kosteneckwerte festzulegen und nicht auf der Basis aktueller Kosten des Netzwerkbetreibers. ${ }^{124}$ Dabei unterstützt das zukunftsgerichtete Konzept Anreize statischer Kosteneffizienz. D.h. es handelt sich um theoretisch minimierte Kosten furr die Produktion von

\footnotetext{
120 Vgl. dazu Schütz/Müller (1999), S. 131.

121 Vgl. RegTP (2001b), S. 63.

122 Solange die Ermittlung von LRIC eine zukunftsgerichtete Perspektive vorweisen, sollte die Basis für die Bewertung eines Assets dem ,Current Cost Accounting' (CCA) unterliegen. Vgl. Valletti (1999), S, $9 \mathrm{f}$.

123 Vgl. Vogelsang (1998), S. 595. Alle Inputs als variabel anzunehmen, ist unrealistisch. Gewisse Inputs müssen bei der Berilcksichtigung eines realistischen Zeithorizonts vorausgesetzt werden können. In der Regel wird die Produktionstechnologie als gegeben angenommen. $\mathrm{Zu}$ Inkrementalkosten vgl. auch Kap. 5.1.3.

124 Vgl. Laffont/Tirole (2000), S. $148 \mathrm{ff}$.
} 
Diensten oder für die Installierung von Netzwerkelementen zu einem gegebenen Zeitpunkt. $^{125}$

Die Bestimmung von langfristigen inkrementellen Kosten für einzelne Netzelemente oder einzelner Dienste ist mit dem ,Total Element Long Run Incremental Cost' - Konzept, kurz TELRIC, möglich. Es geht um die Ermittlung der direkten Stückkosten für den Gebrauch eines Elementes unter Einsatz der effizientesten Technologie. Das TELRIC- Konzept erfaßt langfristige Kosten, die bei Bereitstellung eines zusätzlichen Netzelementes entstehen. Bei der Feststellung der langfristigen inkrementellen Kosten einzelner Elemente werden die zusätzlichen Kosten für die Bereitstellung eines Netzelementes mit einem Zustand, in dem das Element nicht angeboten wird, verglichen. ${ }^{126}$ Das TELRIC- Konzept wird im Rahmen des Unbundlings ${ }^{127}$ häufig diskutiert, weil es für die Bestimmung einzelner Netzelemente eingesetzt wird und dementsprechend dann auch für die Kostenanalyse entbündelter Teilnehmeranschlußleitungen. Es spielt aber ebenso bei der Zusammenschaltung von Netzen eine Rolle.

Das Konzept langfristiger Inkrementalkosten plus einen Gemeinkostenaufschlag wurde in Großbritannien seitens der Oftel entwickelt und fand von 1994/95 bis September 1997 Anwendung. ${ }^{128}$

In den USA wurde seitens der FCC das TELRIC- Konzept 1996 im Telecommunications Act gesetzlich verankert mit Bezug insbesondere auf den Teilnehmeranschlußbereich, da im Fernnetz bereits von funktionsfähigem Wettbewerb ausgegangen wurde ${ }^{129}$. Dabei geht es sowohl um die Ermittlung für Netzzusammenschaltungsentgelte für einzelne Zusammenschaltungsleistungen als auch um die Ermittlung eines monatlichen Entgelts für die Anmietung von Teilnehmer-anschlußleitungen.

Der Europäischen Kommission wurde das TELRIC- Konzept schon 1994 vom WIK im WIK/EAC Report vorgeschlagen. Danach werden in Deutschland seit 1998 Zugangs- und Zusammenschaltungsentgelte sowohl für den Teilnehmeran-

Vgl. Laffont/Tirole (2000), S. 283.

Als Inkrement können ebenso bestimmte Telekommunikationsdienste im Endkundenmarkt zugrunde gelegt werden, also Total Service Long Run Incremental Costs (TSLRIC). Vgl. dazu Sidak/Spulber (1998a), S. 312, 405 ff. TSLRIC sind die vermeidbaren FLC durch Unterbrechung eines vollständigen Dienstes, während die Produktion aller anderen Dienste konstant gehalten wird. Vgl. Dippon (2001), S. 19 f. TSLRIC umschrieb in den USA bis Mitte der 90ger die FL-LRIC als Kostenermittlungsmethode. Mit der Umsetzung des Telecommunication Acts 1996 wurde von der FCC der Begriff durch TELRIC ersetzt Vgl. FCC (1996), S. 320; Winzer (2000), S. 230.

Vgl. Kap. 2.4.2.2.

$\mathrm{Zu}$ einer genauen Zurechnung der ermittelten Telekommunikations-Netzelementkosten auf einzelne Netzzusammenschaltungsleistungen vgl. Oftel (1999), S. 22-23 sowie Winzer (2000), S. $238 \mathrm{f}$.

Vgl. FCC (1999), S. 11/7; Gerpott (1998), S. 135. 
schlußbereich als auch für das Fernnetz bestimmt. ${ }^{130}$ Die Umsetzung des Prinzips erfolgt durch Kostenrechnungssysteme. Sie bzw. ihre Ergebnisse können von der Regulierungsbehörde zur Fundierung von Entscheidungen zur Entgelthöhe im Bereich der Zusammenschaltung, des Zugangs zu Anschlußnetzen und der entbuindelten Teilnehmeranschlußleitungen herangezogen werden. Die Aussagekraft der Ergebnisse hängt von der Güte der eingesetzten Modellparamter ab.

Bei den Kostenmodellen kann auf zwei Modellierungsverfahren zurückgegriffen werden, entweder dem Top-Down- oder dem Bottom-Up- Ansatz. Der TopDown- Ansatz wurde in Großbritannien von der British Telecom in Zusammenarbeit mit der britischen Regulierungsbehörde Oftel entwickelt. Zeitgleich erarbeiteten Industrievertreter zusammen mit Wissenschaftlern im Auftrag der Oftel den Bottom-Up- Ansatz. ${ }^{131}$ Inzwischen verwendet die amerikanische und die deutsche Regulierungsbehörde einen Bottom-Up- Ansatz. Dabei setzt die US- amerikanische Regulierungsbehörde FCC „Cost Proxy Models" für die Bestimmung der Inkrementalkosten ein. Das WIK hat für die deutsche Regulierungsbehörde Kostenmodelle sowohl für das Ortsnetz zur Ermittlung der Kosten für einzelne Netzelemente sowie Teilnehmeranschlußleitungen als auch fur das nationale Verbindungsnetz für die Bestimmung der Kosten für Netzzusammenschaltungsleistungenentworfen. ${ }^{132}$ Im folgenden sollen sowohl der Top-Down- als auch der Bottom-Up- Ansatz näher vorgestellt werden.

\subsubsection{DER TOP-DOWN- ANSATZ}

Der Top-Down- Ansatz wurde von der British Telecom Anfang der Neunziger entwickelt, um die Inkrementalkosten der verschiedenen Netzkomponenten in historischen sowie in aktuellen Kostenverfahren formulieren zu können, mit dem Ziel, den Übergang vom historischen Vollkostenansatz hin zum Ansatz langfristiger Inkrementalkosten zu vereinfachen. ${ }^{133}$ Der historische Vollkostenansatz war bis dahin in Buchhaltungen vieler Unternehmen tief verwurzelt. Oftel antizipierte

130 Die Rechtsvorschriften der EU-Kommission schreiben kein bestimmtes Kostenrechnungssystem vor. Die Kommission empfiehlt allerdings die Festlegung von Zusammenschaltungsentgelten auf Grundlage langfristiger Inkrementalkosten. Vgl. Europäische Kommission (2000), S. 34. Die Europäische Kommission spricht Empfehlungen aus, die die Mitgliedsstaaten zur Auslegung der Zusammenschaltungsrichtlinien heranzuziehen haben und sie so bindet. Vgl. Schütz/Müller (1999), S. 133.

131 Ein systematischer Vergleich der Bottom-Up- mit der Top-Down- Methodik ist zu finden unter Oftel (1996b, 1997a,b).

132 Vgl. WIK $(1998,1999)$.

133 Zur Regulierungspolitik in Großbritannien vgl. unter anderem Armstrong (1997); Cave (1994, 1997), Oftel (1997a); Valletti (1999). 
zu Recht, daß es Zeit brauchen würde, vom historischen Vollkostenansatz auf die Methode langfristiger Inkrementalkosten zu wechseln. ${ }^{134}$

Der Top- Down- Ansatz stellt eine Methode dar, die für die Ermittlung der langfristigen Inkrementalkosten von Diensten bzw. Elementen angewendet werden kann. Die Kosten eines Telekommunikationsnetzes werden ermittelt, indem die Kosten des Betreibers auf die jeweiligen Netzelemente zugeordnet werden. Dabei wird folgendermaßen vorgegangen: Zuerst werden die Gesamtkosten auf die einzelnen Dienste bzw. Netzelemente in homogene Kostenkategorien aufgespalten. Dabei stammen die Kostengrößen aus den vorliegenden Rechnungsdaten des etablierten Unternehmens. Ausgegangen wird von den tatsächlich angefallenen gesamten Kosten aus dem Rechnungswesen, d.h. es wird auf vergangenheitsorientierte Kosten zurückgegriffen. Dabei wird von der vorhandenen Netzarchitektur des Incumbents einschließlich aller gegebenenfalls vorliegenden, historisch gewachsenen Ineffizienzen ausgegangen. Als nächstes wird versucht, die jeweiligen Kostenverursacher zu identifizieren. Schließlich werden die Relationen zwischen den Kostenverursachern und den Kosten der jeweiligen Kategorien mit Hilfe von technisch- ökonomischen Analysen ermittelt. Diese beruhen auf Ingenieurmodellen, wie sie auch im Bottom-Up- Ansatz verwendet werden. ${ }^{135}$ Mit Hilfe dieser technisch-ökonomischen Analysen werden die Kosten immer weiter aufgeschlüsselt, bis sie einzelnen Diensten bzw. Netzelementen zugerechnet werden können. Dabei werden dann die inkrementellen Kostenanteile aus den einzelnen Kategorien je Kostenkomponente aufsummiert.

Da die Ergebnisse des Top-Down- Ansatzes vergangenheitsbezogen sind, ist eine Umwandlung von historischen Kosten in aktuelle Werte in Form von Kostenanpassungen erforderlich, d.h. die Kosten alter Anlagen werden zum Zeitpunkt der Modellierung mit ihren Wiederbeschaffungszeitwerten geschätzt (,Current Cost Accounting' (CCA)). Da eine solche Umstellung relativ umfangreich und aufwendig ist, erfolgt sie pauschal und hochaggregiert. ${ }^{136}$

Die Anwendung des Top-Down- Ansatzes ist insofern vorteilhaft, als Daten für die Modellierung für die jeweiligen Unternehmen leicht und schnell verfügbar sind, weil sie aus dem Rechnungswesen stammen. Ein weiterer Vorteil ist, daß dieser Ansatz die Komplexität der Kostenstrukturen erfaßt. Zum einen werden sowohl direkte als auch indirekte Effekte von Kostenveränderungen abgebildet, ${ }^{137}$

\footnotetext{
134 Vgl. Valletti (1999), S. 10.

135 Vgl. Vogelsang (1998), S. 596.

136 Vgl. Vogelsang (1998), S. 596; Doll/Wieck (1998), S.282. Nach dieser Modellierung können die Inkrementalkosten die Vollkosten sogar übersteigen, weil sie im Grunde die Stand-Alone-Kosten darstellen. Vgl. Vogelsang (1998), S. 596. Zu SAK vgl. auch Kap. 5.1.4.

137 Direkte Effekte beziehen sich auf Veränderungen des Outputs eines Produktes bzw. Dienstes aufgrund der Kostenveränderung; d.h. eine Anpassung der Kostenallokation erfolgt bei einer Änderung des Outputs automatisch. Indirekte Effekte beziehen sich auf Veränderungen ande-
} 
d.h. daß die Auswirkungen der Änderung einer Kostenart auf andere Kostenarten ebenfalls durch die Modellsystematik dargestellt werden. Zum anderen wird eine gewisse Pfadabhängigkeit der Anlageninvestitionen berücksichtigt, da beim Ausgangspunkt der Analyse die tatsächlich vorhandenen Anlagen für die Kostenbestimmung veranschlagt wurden. Das mag auch sinnvoll sein, da der Telekommunikationssektor durch hohe irreversible Kosten seitens des Incumbents gekennzeichnet ist. Es besteht allerdings das Problem, daß aufgrund der Berücksichtigung der historisch gewachsenen Anlagebestände sämtlich damit einhergehende Ineffizienzen in die Kostendatenermittlung miteinfließen. ${ }^{138}$ Dies hat eine systematische Überschätzung der ökonomischen Kosten zur Folge, insbesondere für nicht abschreibungsbedingte Betriebskosten, bei denen keine Anpassung für neu und moderne Anlagen vorgenommen wurden. ${ }^{139}$ Ein weiteres Problem ist, daß für Außenstehende wie z.B. eine Regulierungsbehörde die erforderlichen Kostendaten schwer zu ermitteln und aufgrund der Komplexität schwer nachvollziehbar sind. Aus diesem Grund werden Vorgaben bestimmter Richtlinien der Rechnungslegung gemacht, die das Problem allerdings aufgrund einer asymmetrischen Informationsverteilung $z$ wischen Regulierer und Unternehmen nur unzureichend lösen können. ${ }^{140}$ Der große diskretionäre Spielraum bei der Wahl der Gemeinkostenverteilung führt $\mathrm{zu}$ weiteren Ungenauigkeiten. ${ }^{141}$ Außerdem kann nicht davon ausgegangen werden, daß das Unternehmen zu minimal möglichen Kosten produziert hat. Denn fur das regulierte Unternehmen bestehen durchaus Anreize, Kosten erhöht auszuweisen, um folglich höhere Entgelte und damit auch höhere Endproduktpreise setzen zu können. ${ }^{142}$

\subsubsection{DER BOTTOM-UP- ANSATZ}

Der Bottom-Up- Ansatz greift einen Teil der Kritik an der Top-Down- Methodik auf. So sollen die höheren Kosten aufgrund von Ineffizienzen aus getätigten Investitionen, die bedingt durch den Rückgriff auf Datenmaterial aus der Buchhaltung in die Kostenanalyse in das Top-Down- Verfahren mit einfließen, reduziert werden, indem die Verwendung von Wiederbeschaffungswerten zum Zeitpunkt der

rer Unternehmensbereiche (Personal, Transport, Informationsverarbeitung,...) bedingt durch Kostenveränderungen. Vgl. Oftel (1996a), S. i, S. 93.

Vgl. WIK (1998) S. 1.

139 Vgl. Vogelsang (1998), S. 596; Oftel (1996a), S. ii, 27, 95 f.

140 Vgl. hierzu konkrete Ausführungen in Kap. 6.

141 Vgl. Valletti/Estache (1999), S. 37 f. sowie Kap. 5.4.4 zur Darstellung der Allokation von Gemeinkosten.

142 Vgl. hierzu grundsătzliche Kritik an kostenorientierten Regulierungsansätzen in Kap. 5.4.5. 
Modellierung in eine zukunftsorientierte Bewertung umgewandelt wird. D.h. es wird sich an den Kosten eines fiktiven effizienten potentiellen Newcomers orientiert, der über umfassendes Wissen über das beste einsetzbare Produktionsverfahren verfügt und dementsprechend die aktuell effiziente Technologie anwendet. ${ }^{143}$ Dabei werden im Gegensatz zu den ex post Entscheidungen bei dem Top-DownVerfahren entscheidende Parameter, wie z.B. die verwendete Technik, Lage der Vermittlungsstellen oder Konzentratoren im Ortsnetz, ex ante festgelegt.

Bei der Bottom-Up- Methode handelt sich um Modellrechnungen zur Bestimmung der Produktionsprozeßkosten. Die Ermittlung der Kosten eines Telekommunikationsnetzes erfolgt über die summarische Erfassung der Kosten der einzelnen Netzelemente. Die Netzbetriebs- sowie gegebenenfalls allgemeine Administrationskosten des Netzbetreibers werden dabei noch hinzugerechnet.

Die Bottom-Up-Methode ist im Gegensatz zum Top-Down- Ansatz nicht auf die Topologie eines schon bestehenden Netzes ausgerichtet. Stattdessen wird eine Neuplanung des Telekommunikationsnetzes durchgeführt, indem aus den einzelnen Netzkomponenten eine für den jeweiligen Einsatzzweck möglichst effiziente Netzstruktur entworfen wird. Hier kommen bei der Analyse drei mögliche Netztopologien in Frage: ${ }^{144}$

,New'; d.h. die Netzstruktur wird von Grund auf neu geplant. Es werden Kosten für Teilnehmerverbindungen errechnet, die in einem völlig neu optimierten Umfeld entstehen würden. Diese Herangehensweise wird auch als, Greenfield- Ansatz' bezeichnet, da quasi von Investitionen auf einer, grünen Wiese' ausgegangen wird. ${ }^{145}$ Der Ausgangspunkt ist eine effiziente Anzahl an Vermittlungsstellen und Leitungen unabhängig von dem derzeit bestehenden Telefonnetz (,scorched earth') ${ }^{146}$. Dieser Ansatz findet in der Regel keine Anwendung, solange zusätzliche Kosten des Netzausbaus bei bestehender Topologie geringer sind als Kosten eines Neubaus einschließlich Kosten der Optimierung der Netztopologie. Die Wahl dieses Ansatzes liegt vor allem dann nahe, wenn das gegenwärtige Netz deutliche ineffiziente Strukturen aufweist.

,Rebuild'; d.h. ausgehend von der bestehenden Netztopologie werden bestehende Netze vollständig erneuert und durch neue Einrichtungen ersetzt.

,Upgrade'; d.h. bestehende Netze werden ausgebaut bzw. teilweise erneuert. Dabei verbleiben Teile der bestehenden Infrastruktur in Gebrauch.

Die Szenarien ,Rebuild' und ,Upgrade' finden sich in dem ,scorched node' Ansatz wider. Beim ,scorched node' Ansatz erfolgt eine Berechnung der Zusatzkosten auf der Basis der aktuellen Netztopologie und wendet sich gegen einen völlig

\footnotetext{
143 Vgl. WIK (1998), S. 1 und S. 3 ff.

144 In Anlehnung an Albach/Knieps (1997), S. 20.

14 Vgl. Schütz/Müller (1999), S. 132.

146 Vgl. NERA (1998), S. 1 f.
} 
neuen Netzaufbau. Dabei wird von der Anzahl an Vermittlungsstellen und Leitungen, die zum gegenwärtigen Zeitpunkt der Studie das tatsăchliche Telefonnetz bestreiten, ausgegangen. Es läßt aber offen, ob Zusatzkosten auf der Basis von ,Upgrade' oder ,Rebuild' berechnet werden. ${ }^{147}$

Entscheidend für einen ökonomischen fundierten zukunftsgerichteten Ansatz sind die Ermittlung von Kosten für Teilnehmerverbindungen, die in der Zukunft tatsächlich investiert werden. Bei dem Bottom-Up- Ansatz sind folgerichtig der Ausgangspunkt eine moderne Technologie, effizienzorientierte Netzplanung und aktuelle Inputpreise, bei denen es um die Ermittlung des Investitionsvolumens fur bestimmte Anlagen bei geschätzter zukünftiger Nachfrage geht. Das ermöglicht eine zukunftsgerichtete Kostenrechnung.

\subsubsection{BOTTOM-UP- KOSTENMODELLE IN DER PRAXIS}

Es sollen hier kurz die bekanntesten Bottom-Up- Kostenmodelle aufgezeigt werden. Dabei soll die Funktionsweise eines Bottom-Up- Verfahrens an dem in Deutschland zugrunde liegende Modell etwas näher vorgestellt werden.

Das LECOM-Modell (Local Exchange Cost Optimization Model) als eines der ersten Kostenmodelle hat einen eher wissenschaftichen Hintergrund und stellt basierend auf Ingenieurstudien einen prozeßanalytischen Ansatz dar. Es wurde von Gabel und Kennet ${ }^{148}$ entwickelt und stellt die Grundlage für die vom WIK für die deutsche Regulierungsbehörde konzipierten analytische Kostenmodelle dar. ${ }^{149}$ Inzwischen spielt das LECOM- Modell in der aktuellen Diskussion nur noch ein geringe Rolle. Seine wesentlichen Elemente sind in das neuere Hybrid Cost Proxy Model eingeflossen. ${ }^{150}$

In den USA wurde 1996 eine öffentliche intensive Debatte zwischen der FCC und betroffenen Netzbetreibern über Kostenmodelle geführt. Dabei entwickelten verschiedene Netzbetreiberallianzen Kostenmodelle, die zur Diskussion standen. Folgende Bottom-Up- Modelle, die fur den US amerikanischen Telekommunikationsmarkt von betroffenen Unternehmen entwickelt wurden, sollen hier kurz vorgestellt werden: ${ }^{151}$ Das Benchmark Cost Model von Sprint in Kooperation mit US West und das Cost Proxy Model von Pacific Telesis/Pacific Bell wurden zum

\footnotetext{
147 Vgl. Albach/Knieps (1997), S. 20.

148 Für eine ausfuhrliche Diskussion des LECOM Modells vgl. Gabel/Kennet (1991) sowie (1994), S. 382-396; und auch (1997), S. 411-422.

149 Vgl. Ickenroth/Schmidt (1997), S. 1 f.

150 Als damalige Alternativen zum LECOM Modell standen das Network Development Model von Sharma/Brunnel Vgl. dazu Vogelsang-Studie (1996) sowie eine Studie von Mitchell/Rand (vgl. dazu Mitchell (1990)) zur Debatte. Vgl dazu Albach/Knieps (1997), S. 27 f.

151 Vgl. hierzu auch Doll/Wieck (1998a), S. 281-83; Winzer (2000), S. 268.
} 
Benchmark Cost Proxy Model zusammengefaßt, dessen Inhalte später wiederum in das Hybrid Cost Proxy Model eingeflossen sind.$^{152}$

Ein drittes Modell ist das Hatfield/HAI Model, das von Hatfield im Auftrag von $A T \& T$ und $M C I 1998$ entwickelt worden ist. ${ }^{153}$ Inzwischen ist es vielfach weiterentwickelt worden, da die Modell- Software auf konkrete Daten von Anschlußnetzen einzelner US- Bundesstaaten zurückgeht.

Das schon erwähnte Hybrid Cost Proxy Model (HCPM) wurde schließlich im Auftrag der amerikanischen Regulierungsbehörde FCC entwickelt und greift mit dem Anspruch, das umfassenste Modell zu sein, Elemente aus den drei genannten Kostenmodellen auf.

Die bei der deutschen Regulierungsbehörde zugrunde gelegten analytischen Kostenmodelle sind 1998 für das Ortsnetz und insbesondere für die Kostenermittlung von Teilnehmeranschlußleitungen und 1999 für das nationale Verbindungsnetz von dem Wissenschaftlichen Institut fur Kommunikationsdienste (WIK) entwickelt worden. Deren Vorgehensweise soll hier kurz erwähnt werden.

Im ersten Schritt werden Art und Umfang an Leistungen aufgrund von Nachfrageschätzungen nach den jeweiligen Telekommunikationsdiensten im Ortsnetz bzw. im nationalen Verbindungsnetz bestimmt.

Im zweiten Schritt wird die für die Befriedigung der bestimmten Nachfrage sich ergebende Netzstruktur entworfen und optimiert. Dabei wird eine Bestimmung des Investitionsvolumens für Anlagen und Einrichtungen unter der Voraussetzung vorgenommen, daß die Leistungserstellung effizient erfolgt. Hierbei wird unter Verwendung der neuesten Technologie eine kostenminimale Anlagestruktur mittels eines Optimierungsmodells unterstellt. Dabei ist wie schon erwähnt eine regulatorische Grundsatzentscheidung in bezug auf die Wahl der zugrunde gelegten Netztopologie des Referenznetzes zu treffen: Will man eine vollständige Netzoptimierung im Sinn des Greenfield- Ansatzes unterstellen, oder soll eine vollständige Nachbildung bestehender Netze des regulierten Unternehmens bei der Investitionsrechnung vorausgesetzt werden. Dabei wird ein ,scorched node'- Ansatz in den vom WIK $(1998,1999)$ erstellten analytischen Kostenmodellen für die deutsche Regulierungsbehörde zugrunde gelegt, d.h. es ist kein umfassender Optimie-

152 Für eine ausfuhrliche Darstellung des Benchmark Cost Proxy Model vgl. BellSouth u.a. (1998), S. 21-92.

153 Mit dem Ausscheiden von Hatfield aus Hatfield Consulting wurde sowohl die Beratungsfirma als auch das Modell in HAI umbenannt. Für eine ausfuihrliche Darstellung des Hatfield/HAI Models vgl. HAI (1998). 
rungansatz sowohl fürs Ortsnetz ${ }^{154}$ als auch furrs nationale Verbindungsnetz gewählt worden. ${ }^{155}$

Im dritten Schritt wird das ermittelte Investitionsvolumen mit aktuellen Preisen der Investitionsgüter bewertet. Auf diese Weise wird die Anlagestruktur so beurteilt, wie es ein neuer in den Markt tretender Wettbewerber tun würde. ${ }^{156}$ Für die Errichtung der modellierten Netzstruktur notwendige Investitionen und Betriebskosten werden berechnet und auf die einzelnen Nutzungseinheiten bzw. Netzelemente umgelegt und einzelnen Telekommunikationsdiensten zugeordnet, d.h. im Ortsnetz insbesondere den Teilnehmeranschlußleitungen und im nationalen Verbindungsnetz den Netzzusammenschaltungsleistungen (Local-, Single Transitund Double Transit- Interconnection). Zur Bestimmung der Kapitalkosten des eingesetzten Produktivkapitals ${ }^{157}$ wird erstens das Produktivkapital mit Hilfe des Tageswertprinzips bewertet, d.h. es werden die Wiederbeschaffungsszeitwerte von Investitionsgütern, die zukünftig angeschafft werden würden, ermittelt. Zweitens werden für die verschiedenen Kapitalgütergruppen Abschreibungszeiträume und -verfahren festgesetzt. Drittens wird die kalkulatorische Verzinsung für das eingesetzte Kapital berechnet.

Im vierten Schritt wird das Investitionsvolumen mit Wiederbeschaffungszeitwerten bewertet und unter Berücksichtigung von Abschreibungen, kalkulatorischer Verzinsung auf das eingesetzte Produktivkapital und laufende Betriebskosten der Anlagen in annualisierte Kosten umgerechnet, d.h. die Kosten werden auf die jährliche Leistungsnachfrage umverteilt.

Im fünften Schritt werden die jährlichen Kosten für die einzelnen Produkte bzw. Dienste bestimmt, indem die annualisierten Kosten auf die jährliche Leistungsnachfrage verteilt werden. Hierbei handelt es sich um die Kosten einzelner Netzelmente je Verbindungsminute oder für eine jährliche Überlasssung z.B. bei Teilnehmeranschlußleitungen. ${ }^{158}$

154 Vgl. WIK (1998), S. 10 f. Folgende Annahmen sind hinsichtlich bei der Netzplanung relevanten Produktionsverfahren für das Ortsnetz getroffen worden: Standorte von VSts und Konzentratoren werden vorgegeben. Der Ausbau der Anschlußnetze erfolgt als passives Kupferkabelnetz. Die Vermittlung benht auf digitaler Vermittlungstechnik. Das Verbindungsnetz ist in optischer Übertragungstechnik in der plesiochronen digitalen Hierarchie ausgebaut.

135 Die ,scorched node' Annahme liegt unter anderem auch sowohl bei der Oftel (1996a), S. 9 als auch bei der NERA (1998), S. 1 f. zugrunde.

is6 Vgl. WIK (1998), S. 11.

157 Vgl. WIK (1998), S. 11.

158 Vgl. WIK (1998), S. 70. 


\subsubsection{BEWERTUNG DES BOTTOM-UP- ANSATZES}

Die Verwendung des Bottom-Up- Ansatzes bringt folgende Vorteile mit sich. Der Ansatz ist sehr transparent und einfach zu verstehen. Die unterstellte hypothetische Anlagestruktur ist unabhängig von dem zu regulierenden Unternehmen. D.h. es werden keine realen Rechnungsdaten benötigt, da hypothetische Kostendaten, die mit Hilfe von Ingenieurmodellen simuliert werden, als Grundlage für die Kostenbestimmung genommen werden. Daraus folgt beispielsweise, daß der Regulator nicht mehr auf Informationen über die Kostendaten des Incumbents angewiesen ist. Außerdem läßt sich die Methode problemlos auf andere Netzbetreiber übertragen. Da für die Ermittlung der Kosten eine hypothetisch komplette Anlagenstruktur mit der neuesten Technologie und technisch effizienter Produktion unterstellt wird, ist im Gegensatz zum Top-Down- Ansatz keine Umrechnung von historischen auf aktuelle Kosten erforderlich. ${ }^{159}$ Ineffizienzen der bestehenden Anlagenstruktur fließen aufgrund der Verwendung entscheidungsorientierter Kosten nicht in die Kostenanalyse mit ein.

Die Unterstellung einer hypothetischen Netzstruktur bei Bottom-Up- Modellen bringt allerdings auch eine Reihe an Nachteilen mit sich. Zum einen sei die Problematik aufgrund von für die Kostenanalyse vorgenommenen Nachfrageschätzungen erwähnt. Davon abgesehen, daß Nachfrageschätzungen grob und unzutreffend sein können, lassen sich zudem Auswirkungen von Wettbewerb nur schwer auf die Nachfrage prognostizieren. ${ }^{160}$ Das Risiko von Investitionsentscheidungen, die auf jene Nachfragemodellierungen beruhen, trägt wiederum allein das regulierte Unternehmen.

Ein weiterer Nachteil an dem Bottom-Up- Ansatz bedingt durch die unterstellte hypothetische Anlagestruktur ist, daß er dem bestehenden Netzbetreiber, der über Jahre hinweg sein Netz auf- und ausgebaut hat, nicht gerecht wird. Bereits getätigte irreversible Investitionen werden seitens des etablierten Anbieters nicht berücksichtigt, bzw. es wird unterstellt, daß sich alte Anlagen jederzeit ausrangieren lassen. Dies ist insbesondere im Telekommunikationssektor völlig unrealistisch.

Die Berücksichtigung einer gewissen Pfadabhängigkeit volkswirtschaftlicher und unternehmerisch sinnvoller Investitionsentscheidungen kann die Diskrepanz zwischen ,idealen ' und tatsächlichen Kosten reduzieren. ${ }^{161}$ Pfadabhängigkeit liegt vor, wenn die Kosten für das Aufrüsten eines Netzwerkes billiger sind, als ein neues Netzwerk aufzubauen. Daraus folgt, daß ökonomisch effiziente Inkremen-

\footnotetext{
159 Vgl. Vogelsang (1998), S. 597.

160 Vgl. Knieps (1998b), S. 601.

161 Vgl. Doll/Wieck (1998a), S. 285; Doll/Wieck (1998b), S. 660 f. sowie Knieps (1998b), S. 600.
} 
talkosten auf Basis der faktischen Netzwerkkosten kalkuliert werden müssen. ${ }^{162}$ Das ,Upgrading' bzw. ,scorched node' an tatsăchlichen Netzstrukturvorgaben kommt der Berücksichtigung von Pfadabhängigkeit am nächsten, wăhrend dagegen der sogenannte ,Greenfield- Ansatz' keine Pfadabhängigkeit bei Investitionsentscheidungen berlicksichtigt. Bei den praktizierten Kostenmodellen von der FCC, der RegTP sowie bei der Oftel wurde allerdings von der bestehenden Netzwerkarchitektur ausgegangen.

Eine Vorgabe der Netzarchitektur im Rahmen einer Kostenbestimmung bleibt ein starker Eingriff in die unternehmerische Freiheit. ${ }^{163}$ Die Bestimmung der neuesten im Sinne von der besten Technologie im Rahmen des Bottom-Up-Modells ist ,ex ante' recht schwierig. Dazu ist ein umfassendes Wissen über Leistungsfähigkeit und über die Kostenaspekte innovativer Technologien notwendig. Dieses Wissen ergibt sich oft erst nach Markteintritt und kann kaum antizipiert werden. Es kann allenfalls geschätzt werden. Dadurch kommt es aber zu Verzerrungen. Überhaupt ist eine Definition der „,besten Technologie“ für alle Marktteilnehmer nicht realistisch. Der Incumbent wird immer eine andere Technologie als beste empfinden im Gegensatz zu dem Newcomer. Der Bottom-Up- Ansatz aber berlicksichtigt nur die Sichtweise des Newcomers. In der Realităt wird es mehrere verschiedene Technologien geben, die furr den jeweiligen Betreiber effizient erscheint. Zudem zwingt der rasante technische Fortschritt in der Telekommunikationsbranche zu einer hohen Dynamik in bezug auf realisierte Technologien. Dabei berücksichtigen Bottom-Up-Modelle nicht, daß getroffenen Annahmen über den Netzaufbau und einzusetzende Technologie nicht für alle Marktteilnehmer gelten. Es findet keine Beachtung, daß alternative Netzbetreiber über heterogen effizient verfügbare Technologien verfügen und dementsprechend auch über unterschiedlich effiziente Netztopologien. ${ }^{164}$

162 Es gibt einen wissenschaftlichen Streit darüber, ob die historisch gewachsenen Netzwerkkosten bestehender Netzwerk-Komponenten identisch mit ,sunk costs' sind oder nicht. Nach Vogelsang (1998), S. 595, hăngen Expansionsentscheidungen von vergangenen Investitionen ab. Vergangene Investitionen sind wiederum gleichzusetzen mit versunkenen Kosten, weil sie nicht ohne weiteres durch Veräußerungen rulckgängig gemacht werden können. Handelt es sich um versunkene Kosten, können sie nicht als zusätzliche Kosten bei zukunftsgerichteter Perspektive auftauchen. Wer also mit Pfadabhängigkeit argumentiert, muß gleichzeitig gelten lassen, daß versunkene Kosten nicht zählen. Dagegen behaupten Doll/Wieck (1998b), S. 660 f., pfadabhăngige Investitionen seien nicht mit , sunk costs' zu verwechseln. Sie begründen ihre Aussage mit dem logischen Umkehrschluß, daß wenn ein Upgrading bestehender Netzelemente lohnend ist, der Verkauf bestehender Anlagen nicht anreizkompatibel ist. Für eine Upgrading-Entscheidung ist der Wert bestehender Einrichtungen entscheidend (der Wert der Anlagen, deren Kosten versunken sind, kann sehr hoch sein).

163 Vgl. Knieps (1998b), S. 602 sowie Veröffentlichungskommission des OVG Münster (2001), S. 551.

164 Vgl. hinsichtlich einer ausführlichen Darstellung alternativer Dienste- und Infrastrukturanbieter Kap. 3.4. 
Außerdem sei explizit darauf hingewiesen, daß der Aussagegehalt der ermittelten Daten von der Qualität und der Vollständigkeit des zugrundeliegenden Modells abhängig ist. ${ }^{165}$

Ferner sei kritisch auf den Umgang mit den Gemeinkosten hingewiesen, die durch die Modellrechnung nicht erfaßt werden. Für die Erfassung dieser Gemeinkosten können Daten aus der Kostenrechnung herangezogen werden. Doch dann gelten hier dieselben Probleme wie beim Top-Down- Ansatz. ${ }^{166}$ Beispielsweise wurden insbesondere in dem von der Oftel verwendeten Bottom-Up- Modell bei der Bestimmung eines Gemeinkostenaufschlages Ergebnisse aus dem Top-Down- Verfahren genommen. Damit wurde auf historische Kosten basierend auf Erfahrungen von British Telecom zurückgegriffen, die am Ende pauschal hinzugefügt wurden, obwohl dies der zentralen Idee der Bottom-Up- Ansätze widerspricht. ${ }^{167}$

Kostenmessungen sind eben keine exakte Wissenschaft. Die gemessenen Kosten variieren je nach Meßmethode bzw. Dateneingabe erheblich. So sollen z.B. das Top-Down- wie das Bottom-Up- Verfahren theoretisch zu denselben Ergebnissen kommen. Beide Ansätze führen allerdings in der Praxis zu unterschiedlichen Resultaten. Das ist zurückzuführen auf unterschiedliche Datengrundlagen und $\mathrm{Be}$ wertungsverfahren, mit denen Kosten ermittelt werden. ${ }^{168}$

\subsubsection{GEMEINKOSTENALLOKATION BEI KOSTENORIENTIERTEN RECHNUNGSANSÄTZEN}

Das Konzept langfristiger zusätzlicher Kosten umfaßt keine Gemeinkosten. Es wird immer einige Kosten bei Vermittlung und Zugang geben, die bei der Produktion mehrerer Güter entstehen und die sich nicht zuordnen lassen. Die Höhe solcher nicht zugeordneten Kosten schätzt Oftel in Großbritannien auf $10 \%{ }^{169}$ Darum ist die Formulierung eines Gemeinkostenaufschlags notwendig. Auch bei der Ermittlung elementbezogener Kosten der Netzinfrastruktur können Gemeinkosten auf der Ebene von Netzelementen vorliegen, z.B. bei den Kosten einer Kabelanlage, die von Hauptkabeln und Ortsverbindungskabeln gemeinsam genutzt werden. Kann kein gemeinsamer Nutzungsmaßstab gefunden werden, sind Verfahren der Gemeinkostenallokation anzuwenden. Die Zuordnung und Identifikati-

\footnotetext{
165 Vgl. Knieps (1998a), S. 4.

166 Vgl. Wöhrl (1999), S. 5 sowie Vogelsang (1998), S. 597. Zu einer ausführlicheren Betrachtung der Allokation von Gemeinkosten vgl. Kap. 5.4.4.

167 Vgl. Vogelsang (1998), S. 597.

168 Vgl. Oftel (1996b), Vogelsang (1998), S. 595. Das WIK spricht von Abweichungen um $11 \%$. Vgl. Ickenroth/Schmidt (1997), S. 1.

169 Vgl. Valletti (1999), S. 11.
} 
on von Gemeinkosten ist bekanntermaßen konzeptionell schwierig. ${ }^{170}$ Die meisten Gemeinkosten variieren langfristig mit der Kapazităt einzelner Netzelemente bzw. mit dem Umfang einzelner Leistungen. Wie der angemessene Zuschlag definiert werden soll, ist völlig offen. ${ }^{171}$ Es kommen verschiedene Verfahren in Frage. Hier sollen zwei Verfahren, nämlich das FDC und das Shapley-Value- Verfahren kurz vorgestellt werden, da sie im Rahmen der Kostenstudien diskutiert werden.

\subsubsection{Das Fully Distributed Cost Pricing- VerfahreN}

Die gängigste Methode ist das Fully Distributed Cost Pricing (FDC)- Verfahren $^{172}$, mit dessen Hilfe Gemeinkosten nach verschiedenen Kriterien auf die einzelnen produzierten Dienste bzw. Netzelemente verteilt werden. Dabei können unterschiedliche regulatorische Aufteilungsschlüssel der Gemeinkosten in Betracht kommen: Es kann eine symmetrische Verteilung der Gemeinkosten - die sogenannte ,Equal Charge Method' - erwogen werden, d.h. Gemeinkosten werden zu gleichen Teilen auf die Dienste bzw. die einzelnen Netzelemente aufgeteilt. Die Gemeinkosten können ebenso im Verhältnis der Einzelkosten der Dienste bzw. Netzelemente gemäß der, Attributable Cost Method' aufgeteilt werden oder aber im Verhältnis des Outputs eines einzelnen Dienstes bzw. Netzelementes zum totalen Output anhand der ,Relative Output Method'. Diese Anwendung macht allerdings nur Sinn, wenn die herangezogenen Outputs gleicher Dimension sind. Werden die Outputs der einzelnen Dienste bzw. Netzelemente in unterschiedlichen Dimensionen gemessen, kann eine Gemeinkostenaufteilung im Verhältnis zu den Umsatzerlösen (,Gross Revenue Method') erfolgen. Im Prinzip gibt es unendlich viele Aufteilungsschlüssel. Die Aufteilung kann sich ebenso an der relativen Nutzung der jeweiligen Produkte orientieren (,additive bzw. usageproportional markup'). Oder aber die Gemeinkosten werden in der gleichen Proportion wie das Verhältnis ihrer Zusatzkosten verteilt (uniform bzw. priceproportional markup) $)^{173}$. Sie haben alle gemeinsam, daß Kostenproportionalität angenommen wird, eine Kostenkausalität aber faktisch fehlt. ${ }^{174}$

Das FDC- Verfahren ist nicht gerade bekannt dafür, eine effiziente verursachungsgerechte Aufteilung der Gemeinkosten vorzunehmen. Das Verfahren besticht vielmehr durch seine Praktikabilität und einfache Anwendung sowie geringen Informationsanforderungen.

\footnotetext{
170 Vgl. dazu Kap. 5.1.3; 5.1.5.

171 Vgl. Schütz/Müller (1999), S. 134

$172 \mathrm{Zu}$ einer ausfuhrlichen Darstellung des FDC-Verfahrens vgl. Braeutigam (1980), S. 182-195; Berg/Tschirhart (1988), S. 91-100; Rabe (1989), S. 151 f.

173 Vgl. Laffont/Tirole (2000), S. 137 f.

174 Vgl. Albach/Knieps (1997), S. 34.
} 
In der Regulierungspraxis ist die ,Equal Charge Method' das häufigste angewendete Verfahren. ${ }^{175}$ Oftel verwendete sie im Zusammenhang mit analytischen Kostenmodellen ${ }^{176}$. Auch bei dem vom WIK entwickelten analytischen Kostenmodell für das nationale Verbindungsnetz findet eine Gemeinkostenallokation in Form eines einheitlichen prozentualen Aufschlags zu den Investitionen statt. Dabei wird auf eine Bewertung von Daten aus dem betrieblichen Rechnungswesen zurückgegriffen. Dies ist trotz eines Widerspruchs zur Bottom-Up- Kostenmodellierung unumgänglich. Hieraus ergibt sich unmittelbar das Problem, daß eine Einsicht in die unternehmensinterne Buchhaltung nötig ist. Außerdem sind die Daten der verschiedenen Netzbetreiber uneinheitlich. Das macht die Ergebnisse nicht direkt vergleichbar. ${ }^{177}$

\subsubsection{DAS SHAPLEY-VALUE- VERFAHREN}

Eine andere Möglichkeit der Gemeinkostenallokation stellt das Shapley-ValueVerfahren dar. ${ }^{178}$ Die Shapley-Wert- Methode ist den spieltheoretischen Verfahren zugeordnet. ${ }^{179}$ Es wird ein Unternehmen unterstellt, das verschiedene Produkte produziert bzw. Projekte durchführt und dabei die Reihenfolge der Fertigung einzelner Güter bzw. durchzuführenden Projekte ungewiß und gleich wahrscheinlich ist. D.h. hier besteht die Annahme, daß jedes Produkt bzw. Projekt in prinzipiell jeder denkbaren Reihenfolge hergestellt werden kann. Je nach Reihenfolge Produktfertigung bzw. Projektrealisierung ergeben sich dementsprechend unterschiedliche zurechenbare Kosten. Für jede in Frage kommende Reihenfolge werden die Kosten ermittelt. Dabei werden dem ersten Produkt bzw. Projekt seine Stand-Alone-Kosten, d.h. sämtliche für alle Produkte bzw. Projekte anfallenden Gemeinkosten, zugerechnet. Jedem weiteren Projekt bzw. Produkt werden dagegen nur noch die bei der Herstellung anfallenden inkrementellen Kosten angerechnet. Die Ermittlung des Shapley-Values ergibt sich aus dem Erwartungswert der einem Produkt bzw. Projekt zurechenbaren Kosten unter der Annahme, daß jede Produkt- bzw. Projektreihenfolge gleich wahrscheinlich ist. Eine Kostenallo-

\footnotetext{
175 Vgl. NERA (1998), S. 4 f.

176 Vgl. Valletti (1999), S. 11.

177 Vgl. WIK (1999), S. 40.

178 Eine ausfuhrliche Darstellung der Shapley-Value- Methode vgl. Roth (1988), S. 1-27; Berg/Tschirhart (1988), S. 124-130; Knieps/Sommer (1988), S. 156 f.

179 Ursprünglich bezieht sich die Kernidee des Shapley-Werts (Vgl. Shapley (1953), S. 307-317) auf ein Gewinnspiel, bei dem jeder Spieler grundsätzlich alle denkbaren Koalitionen eingehen kann und dies zum Vorteil aller Spielteilnehmer sein kann. Unter der Annahme, daß jede mögliche Koalition gleich wahrscheinlich ist, ergibt der Shapley-Wert den Erwartungswert des zusätzlichen Gewinns, den der betreffende Spieler allen Spielteilnehmern erbringt. Vgl. Rabe (1989), S. 154.
} 
kation ist effizient, wenn sich sämtliche Kosten auf die einzelnen Produkte bzw. Projekte umlegen lassen. Eine zentrale Eigenschaft des Shapley-Wertes ist, daß die Durchführbarkeit der einzelnen Projekte bzw. der Herstellung einzelner Produkte anhand der Addition der den einzelnen Produkten bzw. Projekten zugeordneten Shapley-Werte abzulesen ist. ${ }^{180}$ Der Gemeinkostenanteil jedes Projektes bzw. Produktes hängt vom eigenen Kostenanteil ab. Deswegen ist das ShapleyValue- Verfahren auch dort als Kostenallokationsmechanismus denkbar, wo herkömmlichen Gemeinkostenschlüsseln die vergleichbaren Outputmengen fehlen. Von Nachteil ist der große erforderliche Datenumfang für die Ermittlung des Shapley-Values. Die Herleitung der Stand-Alone-Kosten der einzelnen Produkte bzw. Projekte ist insofern kompliziert als für die Ermittlung hypothetische Produktionsprozesse ausgewertet werden müssen. Außerdem steigt der Informationsund Rechenaufwand mit der Projekt- bzw. Produktzahl. Eine hohe Projekt- bzw. Produktzahl kann die Berechnung unlösbar machen. Davon abgesehen müssen alle denkbaren Projekt- bzw. Produktreihenfolgen auf ihre physikalische wie ökonomische Sinnhaftigkeit überprüft werden. ${ }^{181}$

In der Regulierungspraxis wird das Shapley-Value- Verfahren beispielsweise in Deutschland bei dem analytischem Kostenmodell für das Ortsnetz für die nicht mehr zurechenbaren, sogenannten leistungsmengenneutralen Gemeinkosten empfohlen. ${ }^{182}$

\subsubsection{KRITIK AN KOSTENORIENTIERTEN RECHNUNGSANSÄTZEN BZW. AN DER ORIENTIERUNG AN LANGFRISTIGEN ZUSÄTZLICHEN INKREMENTALKOSTEN}

Der Vorteil kostenrelevanter Analysen für die Festlegung der Tarife für Zugang und Zusammenschaltung liegt in Verbesserungen von Rechnungsmethoden und einem tieferen Verständnis von Kostenverursachung und Kostenbeziehungen im Gegensatz zu herkömmlichen Regulierungsansätzen wie dem ehemals praktizierten Vollkostenansatz. Allerdings sind die Kosten einer effizienten Leistungsbereitstellung ein theoretisches Konstrukt. ${ }^{183}$ Sie können, müssen aber nicht den aktuellen Kosten des regulierten Unternehmens entsprechen. In der Regel kommt es entweder zu Kostenunter- oder überschätzungen, was beides verzerrende Auswirkungen auf die Entwicklung des Wettbewerbs hat. Es ist fraglich, ob es überhaupt

\footnotetext{
180 Vgl. Nett (1998), S. 525 ff.

181 Vgl. Nett (1998), S. 528.

182 Vgl. WIK (1998), S 6.

183 Vgl. Doll/Wieck (1998b), S. 661; Vogelsang (1998), S. 594 f.
} 
einen objektiven Maßstab bzw. eine neutrale Instanz gibt, die anhand einer objektiven Methodik die ,wahren' Kosten einer effizienten Leistungsbereitstellung ermitteln kann. Im weiteren soll sowohl auf mögliche Ursachen der Kostenfehleinschätzungen als auch auf die daraus folgenden Konsequenzen näher eingegangen werden.

Eine Ursache für eine falsche Kosteneinschätzung liegt in der notwendigen $\mathrm{Be}-$ rücksichtigung eines Gemeinkostenaufschlags, der insbesondere bei der Anwendung des FDC- Verfahrens zu Ungenauigkeiten führt. ${ }^{184}$

Informationsdefizite machen grundsätzlich eine objektive Bestimmung der ,wahren' langfristigen zusätzlichen Kosten unmöglich. Grund dafür sind die notwendigen Informationserfordernisse zum Beispiel für die Ermittlung der Kosten der Netzwerkausstattung und einzelner Netzelemente. Gewöhnlich werden die Kosten einzelner Netzwerkelemente nach dem Marktpreis bestimmt. Es lassen sich aber nicht fur alle Netzelemente Marktpreise definieren, weil sie zum einen nicht immer am Markt gehandelt wurden. ${ }^{185} \mathrm{Zum}$ anderen hängt der Aufbau einer Netzinfrastruktur von der Vorhersage über den zukünftigen Gebrauch der einzelnen Netzelemente $a b$. Die hängen wiederum von der Vorhersage über die Entwicklung demographischer Faktoren wie Bevölkerungswachstum oder Wirtschaftsentwicklung ab. ${ }^{186}$

Bei der Vorhersage über die Nutzung einzelner Netzelemente besteht zusätzlich ein Bewertungsproblem. In der Telekommunikationsbranche werden in der Regel ganzheitliche Investitionen in die Netzwerkausstattung gemacht. Die können sich dennoch lohnen, auch wenn die Kapazität über einen gewissen Zeitraum nicht voll ausgelastet ist. ${ }^{187}$ Werden Kostendurchschnitte ermittelt, die von einer konstanten Kapazitätsauslastung ausgehen, führen sie aus diesen Gründen zu Verzerrungen. Außerdem gelingt es einer Vorhersage über die Nutzung der einzelnen Elemente nicht, technischen Fortschritt und seine Auswirkungen adäquat zu berücksichtigen. Dynamische Entwicklungen des Marktes führen zu einer schnellen Veralterung und ungenauen Schätzung des zur Verfügung stehenden Datenmaterials.

Bei der Bestimmung langfristiger Inkrementalkosten wird implizit der Einsatz der neuesten Technologie berücksichtigt. Das zu regulierende Unternehmen wird dagegen auf der Grundlage seiner bestehenden und damit älteren Technologie kalkulieren, d.h. daß es und vor allem seine Kreditgeber historische Daten aus dem Rechnungswesen ansetzen. Wirkt sich, wie generell angenommen, der technische Fortschritt kostensenkend aus, fallen die ermittelten LRIC des Unternehmens be-

\footnotetext{
184 Vgl. dazu Kap. 5.4.4.1.

185 Z.B. wenn es nur einen Nachfrager nach Netzelementen gibt oder bei Produkten, die nach individuellen Anfertigungswünschen hergestellt wurden.

$186 \mathrm{Vgl}$. Laffont/Tirole (2000), S. $149 \mathrm{f}$.

187 Vgl. Laffont/Tirole (2000), S. 150.
} 
dingt durch andere Ausgangsdaten höher aus als die von der Regulierungsbehörde ermittelten Inkrementalkosten. Daraus folgt, daß die Regulierungsbehörde dem Incumbent eine Entgelthöhe für den Netzzugang sowie für Netzzusammenschaltung vorschlagen wird, die unter der kostendeckenden Entgelthöhe liegen kann und zu Verlusten seitens des Netzbetreibers fuhren würde. Es kann nicht beabsichtigt sein, daß ein Anbieter, der sein Netz Wettbewerbern zur Verfügung stellt, dadurch Verluste erleidet.

Außerdem besteht das Problem der, Stranded Costs'. Hierbei handelt es sich um Kosten, die dem Incumbent durch in der Vergangenheit getătigte Investitionen zur Erfullung von Regulierungsaufgaben angefallen sind. Ändern sich die Regulierungsauflagen bzw. findet ein Regulierungswechsel statt, können diese Investitionen als ,gestrandet' bezeichnet werden, da sie nach einer Änderung des Regulierungsrahmens im Sinne von versunkenen Kosten nicht veräußert oder niedriger bewertet werden. ${ }^{188}$ Die Investitionen waren zum Zeitpunkt der Aufwendung erforderlich und vielleicht sogar als gesetzliche Auflage unvermeidlich. Folglich war die Entwertung damals unvorhersehbar. ${ }^{189}$ Hătte der Incumbent von der Änderung der Regulierungsauflagen gewußt, hätte er diese Investitionen nicht getätigt. Der Netzaufbau des damals geschützten öffentlichen Monopols in der Telekommunikation erfolgte unter anderen politischen Bedingungen als heute. Dabei lagen ebenso andere Investitionskalküle vor. Es ist fraglich, inwieweit die Liberalisierung des Marktes und ihre Konsequenzen in damaligen Investitionsentscheidungen seitens des Incumbents antizipiert worden ist. Für Wettbewerber, die seit der Liberalisierung auf den Markt getreten sind, fallen diese ,Stranded Costs nicht mehr an. Können sie aufgrund dessen einen geringeren Preis als der Incumbent nehmen, kann es zu technisch ineffizienten Ergebnissen kommen, wenn der Incumbent der eigentlich technisch Effizientere ist, aber den höheren Preis nehmen muß, um seine ,Stranded Costs' decken zu können. ${ }^{190}$ Sind die Folgen einer kostenbasierten Entgeltregulierung eine Kostenunterdeckung, da vergangenheitsbezogene versunkene Kosten nicht berilcksichtigt werden, kann das wiederum Auswirkungen auf zukünftige Investitionsentscheidungen haben, weil dann nämlich die Anreize, zukünftige Investitionen zu tätigen, sinken. ${ }^{191}$

\footnotetext{
188 Vgl. Garcia-Martin (2001), S. 4.

189 Vgl. Baumol/Sidak (1995), S. 839

190 Vgl. Baumol/Sidak (1995), S. 837 f.

191 Vgl Kolbe/Tye (1996), S. 1027f.; Sidak/Spulber (1998b), S. 123 f. Die daraus resultierende Forderung, bei der Regelung des Zugangsentgelts ,Stranded Costs' als Kostenkomponente in Form eines Aufschlags mit einfließen zu lassen, wirft wiederum Probleme bedingt sowohl durch Informationsdefizite in bezug auf vollständige Kosteninformationen als auch durch Informationsasymmetrien auf. Aus diesem Grund schlagen Crew/Kleindorfer (1999), S. $71 \mathrm{ff}$. die Verwendung des Price-Cap- Verfahrens vor, da dann der Regulierer die genaue Höhe der Kosten nicht kennen muß.
} 
Auf der anderen Seite stellt sich ein weiteres zentrales Problem hinsichtlich der Bestimmung der langfristigen zusätzlichen Kosten seitens einer Behörde durch die asymmetrische Informationsverteilung zwischen Regulierungsbehörde und monopolistischem Betreiber. Sie führt dazu, daß das regulierte Unternehmen kurzfristig Interesse hat, Kosten höher auszuweisen, weil es dann ebenso ein höheres Entgelt nehmen kann ${ }^{192}$. Eine ausfuhrliche Analyse dieses Sachverhaltes erfolgt in Kapitel 6.

Es sei explizit darauf hingewiesen, daß nicht grundsätzlich davon ausgegangen werden kann, daß sich eine Institution wie eine Regulierungsbehörde neutral gegenüber allen Marktteilnehmern verhält. Beispielsweise erfolgt die Wahl des Modelldesigns für die Umsetzung von TELRIC aus der Perspektive des Regulierers, d.h. die Entscheidung ist abhängig vom aktuellen Regulierungsstand und orientiert sich nicht am minimalen Regulierungsbedarf. ${ }^{193}$ Eine Regulierung auf Basis der langfristigen inkrementellen Kosten räumt der regulierenden Institution eine Schlüssel-Rolle ein, weil es mit der Bestimmung des Zugangs- bzw. Zusammenschaltungsentgelts Einfluß sowohl auf die Entwicklung des Wettbewerbs als auch auf marktliche Veränderungen, bedingt durch Diffusion von Innovationen und technischem Fortschritt, nimmt. Eine Entgeltbestimmung auf der Grundlage von langfristigen Inkrementalkosten ist darauf ausgerichtet, daß das regulierte Unternehmen bei der Gewährung von Netzzugang keinen Gewinn macht. Dadurch führt sie zu Verzerrungen hinsichtlich des Verhaltens der Marktteilnehmer. Sie provoziert nämlich zum einen Anreize seitens des Netzbetreibers, den Zugang zu seinem Netz gegenüber Wettbewerbern nicht marktneutral anzubieten. Daraus folgt wiederum die Notwendigkeit zusätzlicher regulierender Eingriffe bezüglich der Überwachung des Netzbetreibers. Mit anderen Worten zieht eine Regulierung auf Basis langfristiger Inkrementalkosten weitere Regulierungsschritte nach sich. Ein daraus resultierender, heavy-handed" Regulierungstil verursacht hohe Regulierungskosten (Personal, Informationsermittlung). Außerdem kreiert er einen Verbund an politischen Interessengruppen, die Anreize verspüren, über Parteien Einfluß auf die Regulierungspraxis zu übernehmen. ${ }^{194} \mathrm{Zum}$ anderen beeinflußt es die Investitionsanreize in die bestehende Netzarchitektur des Incumbents, die bei einem eventuell nicht einmal kostendeckenden Netzzugangsentgelts sinken.

Die Regulierungsbehörde beeinflußt durch ihre Entscheidungen nicht nur das Verhalten des Incumbents, sondern auch der Newcomer in bezug auf ihre wettbewerbliche Ausrichtung, nämlich ob sie mehr in eigene Infrastruktur investieren oder mehr Infrastruktur anmieten. Ein niedriges Entgelt auf der Grundlage von

\footnotetext{
192 Vgl. Vogelsang (1998), S. 595.

193 Vgl. Knieps (1998), S. 601. Schließlich unterliegt eine Behörde als Akteur einer gewissen Eigennutzorientiertheit. Vgl. dazu Kap. 6.2.1.

194 Vgl. dazu Kap. 6.2.3.
} 
LRIC wird prinzipiell den Dienstewettbewerb fordern und den infrastrukturbasierten Wettbewerb schwächen. ${ }^{195}$ Als Rechtfertigung für eine Entgeltregulierung wird darauf hingewiesen, daß aufgrund dessen sich nur dort infrastrukturbasierter Wettbewerb entwickeln wird, wo es ökonomisch ,sinnvoll' ist bzw. keine Kostensubadditivităten vorliegen. Diese Argumentation berücksichtigt allerdings nicht, daß technologische Weiterentwicklungen von Substituten Rahmenbedingungen verändern können, so daß Kostensubadditivität in Engpaßbereichen nicht mehr zwangsläufig gegeben ist. ${ }^{196}$

Fazit ist, daß es keine neutrale Instanz sowie keinen objektiven Maßstab zur Erstellung langfristiger zusätzlicher Inkrementalkosten gibt und ihre Ermittlung eine genuin unternehmerische Aufgabe darstellt.

195 Vgl. Cave/Prosperetti (2001), S. 421. Sie zeigen anhand von OECD- Daten auf, daß Infrastrukturinvestitionen in Europa seit der Liberalisierung stagnieren.

Vgl. hierzu Kap. 2.3 und 3.4.3. 
Sarah Eliza Schniewindt - 978-3-631-75130-5 Downloaded from PubFactory at 01/11/2019 07:57:00AM via free access 


\section{POLITÖKONOMISCHE SICHTWEISE}

Bei der Einfuhrung von Wettbewerb im Teilnehmeranschlußbereich galt bislang der Ansatz ,Deregulierung durch Regulierung' als praktikabel. Die Nachteile an der Regulierung von Zugangsentgelten mit Hilfe von theoretischen Ansätzen aus der traditionellen Regulierungsökonomie, wie sie in Kapitel 5 dargestellt worden sind, sind augenscheinlich. Aufgrund von Informationsdefiziten seitens der Regulierungsbehörde verzerren regulatorische Eingriffe ins Marktgeschehen sowohl die Marktstruktur als auch das Marktverhalten des Incumbents wie der Wettbewerber nachhaltig. Die Theorie der ,neuen Regulierungsökonomie ${ }^{1}$ dagegen berücksichtigt die asymmetrische Informationsverteilung zwischen Regulator und reguliertem Unternehmen. Da sich für das Unternehmen Anreize ergeben, Informationen zurückzuhalten, z.B. was die Kostenermittlung betrifft, muß der Regulator also Anreizschemata entwerfen, die eine asymmetrische Informationsverteilung berulcksichtigen. Das Problem der asymmetrischen Informationsverteilung hat die deutsche RegTP in der Regulierungspraxis versucht $\mathrm{zu}$ lösen, indem sie analytische Kostenmodelle zur Bestimmung von Zugangs- und Zusammenschaltungsentgelten herangezogen hat, um bei der Entgeltbestimmung von den Kostenangaben des Incumbents möglichst unabhängig zu sein. ${ }^{2}$ Dabei wird implizit die Neutralität einer regulierenden Instanz vorausgesetzt, die in der Realität aber nicht gegeben sein muß. In diesem Kapitel sollen politökonomische Zusammenhänge in der Regulierungsdiskussion aus der Perspektive des ,Public Choice' beleuchtet werden. Dabei soll berücksichtigt werden, daß eine Regulierungsbehörde keine neutrale Instanz ist, sondern ebenso ein Akteur im wirtschaftspolitischen Geschehen wie alle anderen Betroffenen. Das Zusammenspiel zwischen einer Regierung, einer Regulierungsbehörde, dem regulierten Unternehmen und seinen Wettbewerbern soll anhand der Prinzipal-Agent- Theorie näher analysiert werden. ${ }^{3}$ Dazu wird im folgenden Kapitel 6.1 eine allgemeine und zusammenfassende Einfuhrung in die Prinzipal-Agent- Theorie gegeben, bevor sie in dem darauf folgenden Kapitel 6.2 auf den Telekommunikationsmarkt angewendet wird.

Zur ,Neuen Regulierungsökonomie‘ vgl. Laffont/Tirole (1994a), S. 34 f.

Vgl. dazu Kap. 5.4.3.

Zu einer ausfuhrlichen Darstellung der Prinzipal-Agent- Theorie vgl. Laffont/Martimort (2002). Zur Anwendung der Prinzipal-Agent- Theorie auf Regulierungsprobleme vgl. Baron/Myerson (1982), Laffont/Tirole (1986), rep. in (1994a), S. 475 ff. Zur Anwendung von ,Mechanism Design Models‘ auf die Regulierung natürlicher Monopole vgl. Noth (1994) . Es handelt sich bei den Mechanism Design Modellen um Versuche, allokative Effizienz trotz asymmetrischer Information und Interessenkonflikten und Entscheidungen unter Unsicherheit zu erzielen. Vgl. Noth (1994), S. 28. 


\subsection{Themenrelevante Grundzüge Der Prinzipal-Agent- THEORIE}

Als Prinzipal wird der Auftraggeber bezeichnet, der einen informierten Beauftragten, also einen Agenten, delegiert, eine Leistung zu erfüllen, die er mangels Zeit und/oder mangels Fachkompetenz nicht selbst erfüllen kann. Dabei kann der Auftraggeber in der Regel die vom Agenten erbrachte effektive Leistung bzw. die Leistungskriterien nicht wirklich beurteilen.

Klassische Beispiele für ein Prinzipal-Agent- Verhältnis sind ${ }^{4}$

- das Patient-Arzt- Verhältnis, wobei der Patient als Prinzipal den Arzt als Agenten mit der Behandlung seiner Krankheiten beauftragt ${ }^{5}$,

- das Verhältnis zwischen Versicherer (Prinzipal) und Versicherungsnehmer (Agent) ${ }^{6}$,

- das Aktionärs-Manager- Verhältnis, bei dem der Aktieneigentümer als Prinzipal den Manager als Agenten die Entscheidung einer optimalen Unternehmensführung überträgt ${ }^{7}$

- oder die Übertragung von Diensten seitens der gewählten Regierung (Prinzipal) auf den öffentlichen Sektor (Agent). ${ }^{8}$

Im Rahmen der Prinzipal-Agent- Theorie sind drei zentrale Merkmale konstitutierend: Zum einen ist es die asymmetrische Informationsverteilung, zum anderen sind es Interessenkonflikte zwischen Prinzipal und Agenten. Entfällt eine der beiden Bedingungen, erübrigt sich damit auch das Prinzipal-Agent- Problem. Wäre eine symmetrische Informationsverteilung gegeben, dann könnte der Prinzipal den Agenten perfekt überwachen. Oder aber läge eine Interessenharmonie vor, dann wäre eine Steuerung des Agenten überflüssig. ${ }^{9}$ Zum dritten handelt es sich um eine Situation unter Unsicherheit. Ein Vertrag zwischen Prinzipal und Agent verteilt die Risiken aufeinander.

Die asymmetrische Informationsverteilung zwischen dem Auftraggeber und dem eingesetzten Agenten beruht auf zwei Elemente, die sich gegenseitig begünstigen. Zum einen ist sie darauf zurückzuführen, daß der Agent sowohl vor als auch nach Vertragsabschluß Zugang zu Informationen hat, zu denen der Prinzipal

\footnotetext{
Weitere Beispiele sind bei Kiener (1990), S. 21 und Noth (1994), S. 19 zu finden.

Vgl. hierzu im Rahmen der Gesundheitsökonomie u.a. Arrow (1991), S. 38; Breyer/Zweifel (1997), Pfaff/Zweifel (1998), S. 189 f.

6 Vgl. Arrow (1991), S. 39; Spence/Zeckhauser (1971); Rothschild/Stiglitz (1976).

7 Vgl. hierzu im Rahmen von Unternehmenstheorien Holmström/Milgrom (1991); Pfaff (1994/1996).

8 Vgl. hierzu im Rahmen des New Public Management Dunleavy/Hood (1994); Hood (1995), Pfaff/Zweifel (1998), S. 188 f.

$9 \quad$ Vgl. Pfaff/Zweifel (1998), S. 187 f.
} 
keinen hat und nicht einmal von der Existenz weiß. Man spricht in diesem $\mathrm{Zu}$ sammenhang auch von, hidden information' bzw. ,hidden knowledge ${ }^{10}{ }^{10}$ Nach Vertragsabschluß kann der Prinzipal aufgrund der ihm fehlenden Informationen nicht beurteilen, ob die Handlungen des Agenten in seinem Sinne sind." Zum zweiten kann der Prinzipal die Handlungen des Agenten nicht direkt beobachten ,hidden action '. ${ }^{12}$ Er kann also nicht hinreichend überprüfen, ob der Agent seine Arbeit hinsichtlich der Qualität der Entscheidungen im Sinne des Prinzipals erfüllt (,ex ante moral hazard'). Folglich kann der Prinzipal vor wie nach Vertragsabschluß die Eigenschaften und Fähigkeiten des Agenten nicht hinreichend beurteilen, d.h. ob der Agent überhaupt qualifiziert für die Erbringung der erforderlichen Leistung ist. ${ }^{13}$ Wenn nach der Verhaltensentscheidung seitens des Agenten ein Ergebnis realisiert wurde und Informationsasymmetrie aufgrund des risikobehafteten Ergebnisses bestehen bleibt, ergeben sich Anreizprobleme im Sinne von ,ex post moral hazard'. D.h. selbst wenn der Prinzipal weiß, wie sich der Agent verhalten will und wird, kann das richtige Verhalten aufgrund von fehlender Verifizierbarkeit nicht durchgesetzt werden. ${ }^{14}$

Die Prinzipal-Agent Beziehung läßt sich im folgenden konkretisieren. Der Prinzipal beauftragt einen Agenten fur die Erfüllung einer Leistung, indem beide einen Kooperationsvertrag eingehen. Beide Vertragspartner handeln eigennutzorientiert. Dabei unterliegt das Verhalten des Prinzipals in der Regel einer gewinnorientierten Maxime. Dazu sollte ihm seine Zahlungsbereitschaft in bezug auf die erfullte Leistung bekannt sein. Er wägt die Erträge aus der erfullten delegierten Leistung mit den anfallenden Kosten ab. Das Verhalten des Agenten unterliegt wiederum dem Prinzip, den eigenen Nutzen zu maximieren. Sein Nutzen ist von zwei Faktoren abhängig: zum einen vom Einkommen, das unabhängig von der Leistungserbringung erfolgt, zum anderen von einer nicht-monetären Entlohnung in Form von ,Shirking'. Es wird also unterstellt, daß er bei der Leistungerfullung so etwas wie Arbeitsleid empfindet. Unter dem Kalkul seiner Nutzenmaximierung bearbeitet er den Auftrag und erwirtschaftet ein Ergebnis. Das Ergebnis ist zu Beginn bei Abschluß des Vertrages noch unsicher. Die Qualităt des Ergebnisses hängt nicht nur vom pflichterfullenden Einsatz des Agenten ab, sondern auch vom $\mathrm{Zu}$ -

10 Vgl. Arrow (1991), S. 39.

"Im Zusammenhang mit dem Begriff, hidden information' fallt hăufig der Begriff, adverse selection', der sich auf eine asymmetrische Informationsverteilung zwischen Marktteilnehmern vor Vertragsabschluß bezieht. Vgl. Akerlof (1970), Kiener (1990), S. 24 f., Noth (1994), S. 22.

12 Vgl. Arrow (1991), S. 38.

13 Dies wird auch als, hidden characteristics ' von Picot (1991), S.152 bezeichnet.

14 Vgl. Rühl (2001), S. 30. Als Beispiel sei hier eins aus der Sozialversicherung genannt: Eine Person, die in eine Krankenversicherung einzahlt, findet, daß ihr eine Kur zustünde, die sie nicht machen wïrde, wenn sie sie selbst bezahlen müßte. 
fall bzw. von Umwelteinflüssen. Es ist anzunehmen, daß je größer das Engagement des Agenten ist, desto höher die Wahrscheinlichkeit eines gehaltvollen Ergebnisses ist. Allerdings kann ein durchaus engagierter Einsatz durch ein zufälliges Ereignis zunichte gemacht werden, oder aber die Faulheit eines Agenten (Shirking) kann ebenso durch ein zufälliges Ereignis kompensiert werden. ${ }^{15}$ Für den Auftraggeber, dem das Ergebnis vorliegt, ist es nicht ersichtlich, in welchem Maße die erfüllte Leistung auf das Engagement des Agenten oder auf den Zufall zurückzuführen ist. Da die Leistungserbringung unabhängig vom Einkommen ist, kann der Agent im Grunde nur über nicht-monetäre Entlohungen im Sinne von ,Shirking' seinen Nutzen maximieren. Hierauf beruht im wesentlichen der Interessenkonflikt zwischen Agenten und Prinzipal.

Sowohl die asymmetrische Informationsverteilung als auch der Interessenkonflikt führen zu Gewinneinbußen auf Seiten des Prinzipals. Will er jene minimieren, muß er den Agenten stärker kontrollieren, was wiederum zusätzlich Kosten verursacht. Zudem fallen Kosten an, wenn es zu Sanktionen aufgrund von entdecktem Fehlverhalten kommen sollte. Alle in diesem Zusammenhang anfallenden Kosten werden im allgemeinen auch als Agency- Kosten bezeichnet. Eine Möglichkeit, Agency- Kosten zu minimieren, bestünde in der Veränderung von Anreizmechanismen z.B. in Form einer anreizkompatiblen Vertragsgestaltung. Das hieße, daß die Leistungserbringung ans Einkommen beispielsweise in Form einer Gewinnbeteiligung gekoppelt werden sollte. ${ }^{16}$

\subsection{Prinzipal, Agentur Und Agent in der TELEKOMMUNIKATION}

Es sollen im folgenden die Grundzüge der Prinzipal-Agent- Theorie erweitert ${ }^{17}$ und auf die politische und regulatorische Entscheidungsfindung im Telekommunikationsmarkt angewendet werden. Dabei sollen alle betroffenen Parteien als Akteure gesehen werden und Interaktionen zwischen ihnen als strategische Spiele interpretiert werden. Es spielen neben dem Incumbent seine Wettbewerber, die Regulierungsbehörde sowie die Regierung mit.

Der Prinzipal repräsentiert in diesem Fall die vom Volk gewählte Regierung. Seinem Kalkül unterliegt zunächst das Wohl aller zu sichern und zu fördern. Aus

is Zudem spielt bei der Qualität des Ergebnisses der Grad der Risikoaversion des Agenten eine Rolle, was insbesondere in der Analyse von Versicherungsmärkten von großer Bedeutung ist. Vgl. Gravelle/Rees (1992), S.719 ff.

$16 \mathrm{Pfaff} / Z$ weifel (1998), S. 189 weisen auf Schwierigkeiten der Umsetzung einer leistungsorientierten Entlohnung insbesondere im öffentlichen Sektor hin.

17 Vgl. Laffont/Tirole (1994a), S. 475 ff. 
dem Grund, daß ein Telefonanschluß zur öffentlichen Grundversorgung gehöre, hatte er ursprünglich den Aufbau und die Instandhaltung eines Telekommunikationsnetzes in die Hände eines öffentlichen Monopols - den Agenten - gelegt. Da inzwischen eine nahezu vollständige Netzabdeckung innerhalb der Bevölkerung realisiert worden ist, hat sich die Definition des Wohls aller verändert. Dabei ist in den Vordergrund getreten, daß Telekommunikationsdienste möglichst preiswert bereitgestellt werden sollen. In diesem Zusammenhang verfolgt der Prinzipal ganz konkret mit der Liberalisierung des ehemals staatlich geförderten monopolisierten Marktes das Ziel, funktionsfähigen Wettbewerb zu etablieren. Er verspricht sich davon eine erhöhte Zahl zufriedener Wähler und damit eine erhöhte Chance auf eine Wiederwahl. ${ }^{18} \mathrm{Da}$ der Prinzipal nicht über die notwendige Zeit und Fachkompetenz verfügt, den Liberalisierungsprozeß zu begleiten, schaltet er zwischen sich und dem Agenten eine Agentur als weitere Instanz. Als solche wird eine sektorspezifische Regulierungsbehörde von ihm beauftragt, funktionsfähigen Wettbewerb in dem Markt zu errichten. Diese Behörde soll über ausreichend Zeit, Ressourcen und Erfahrung verfügen, die notwendigen Informationen über Marktstruktur, Marktverhalten und Marktergebnis zu sammeln und auszuwerten. Um wirksamen Wettbewerb zu erreichen, obliegt es ihr, den Agenten als ehemaliges staatliches Monopol und marktbeherrschendes Unternehmen zu regulieren. Dabei ist das Verhältnis zwischen Agentur und Agent mit den gleichen Merkmalen wie die vorangegangene Beschreibung der Prinzipal-Agent- Beziehung gekennzeichnet. Bei der Umsetzung der Aufgabe, Wettbewerbern einen Markteintritt im Telekommunikationsmarkt zu ermöglichen, erzwingt die Agentur von dem Agenten, den Zugang zu seinem Netz kostenorientiert bereitzustellen. Dabei ist insbesondere beim LRIC Konzept kein Gewinn vorgesehen. Bei der Regulierung von Entgelten für den Zugang und die Zusammenschaltung von Netzen verlangt die Agentur eine detaillierte Aufschlüsselung der anfallenden Kosten. ${ }^{19}$ Da der Regulierungsbehörde die asymmetrische Informationsverteilung zwischen Agentur und Agenten und die daraus entstehenden Anreize seitens des Agenten bekannt sind, ist es deswegen notwendig, ein Regulierungsdesign zu entwerfen, das möglichst wenig auf Informationen des regulierten Unternehmens angewiesen ist. Aus diesem Grund hat sich die deutsche Regulierungsbehörde furr den Gebrauch analytischer Kostenmodelle entschieden, die Netzzugangspreise weitestgehend auf der

18 Im Grunde darf nicht vergessen werden, da $B$ sich der hier definierte Prinzipal letztenendes aus Regierungsmitgliedern konstituiert, die die Interessen des Volkes vertreten sollen. Mit anderen Worten laBt sich auch hier eine Prinzipal-Agent- Beziehung formulieren, wobei die Regierungsmitglieder als Agenten beauftragt werden, die Interessen der Bürger zu vertreten. Folglich sind Regierungsmitglieder primär eigennutzorientiert und stellen zudem ein Ziel der Einflußnahme von Interessengruppen dar. Dies soll zunächst ausgeblendet werden, da hier die

Politik der Regulierungsbehörde fokussiert werden soll.

Vgl. dazu 5.4. 
Kostenstruktur von hypothetischen Netzen zu ermitteln und nicht auf tatsächliche Kosten des regulierten Unternehmens. ${ }^{20}$ Bei der Leistungserfullung seitens der Agentur darf nicht vernachlässigt werden, daß sie ein eigenes Verhaltenskalkül bei ihren Entscheidungen hat. Die Beziehung zwischen Prinzipal und Agentur entspricht ebenso der beschriebenen Prinzipal-Agent- Beziehung. Diese soll im folgenden Kapitel näher betrachtet werden.

\subsubsection{VERHALTENSKALKÜl DER AGENTUR}

Im folgenden soll auf drei Schlüsselcharakteristika einer Agentur eingegangen werden, die sich gegenseitig bedingen: Zum einen handelt eine Agentur eigennützig. Zum anderen basiert ihr Verhalten auf dem Prinzip der Budgetmaximierung. Zum dritten kann sie von Interessengruppen beeinflußt werden.

Im Rahmen der zu lösenden Aufgabe handelt die Regulierungsbehörde eigennützig. Dies betrifft primär den Selbsterhalt als Institution, d.h. ihre Existenz muß dauerhaft legitimierbar sein. Dieses Kalkül wird ihre Entscheidungen in bezug auf regulatorische Eingriffe in den Markt maßgeblich beeinflussen. Mit anderen Worten ist sie gar nicht daran interessiert, dauerhaft Wettbewerb zu etablieren, weil ihre Existenz mit dem Bestehen von wirksamen Wettbewerb nicht mehr notwendig ist. Strategische Interaktionen mit anderen Akteuren, die ihre langfristige Präsenz sichern sollen, untermauern diese These. ${ }^{21}$ Das Interesse am Selbsterhalt einer Behörde stellt einen handfesten Interessenkonflikt zwischen Prinzipal und Agentur dar.

Gegenüber dem herkömmlichen Prinzipal-Agenten- Verhältnis, wie es in Kap. 6.1 dargestellt worden ist, bei dem das Verhalten der Agentur dem Kalkül der Nutzenmaximierung unterliegt, kommt hinzu, daß es sich hier bei der Agentur um eine öffentliche Behörde handelt, deren Verhaltenskalkül von einer Budgetmaximierung geprägt ist. Wie im klassischen Budgetmaximierungsmodell nach Niskanen $^{22}$ liegt hier ein bilaterales Monopol vor, d.h. es gibt nur einen Anbieter - also die Behörde -, und es gibt nur einen Nachfrager - den Prinzipal, der die Einführung von Wettbewerb im Telekommunikationsmarkt als von der Behörde bereitgestelltes Gut nachfragt. ${ }^{23}$ Mitglieder einer Behörde haben folgende Ziele im Auge: ${ }^{24}$ Neben dem Erhalt ihres regulären Gehalts, das im übrigen unabhängig

\footnotetext{
Vgl. hierzu ausfuhrlich Kap. 6.4.3.1.

Vgl. dazu Kap. 6.3.

Vgl. Niskanen (1968), S. 293 ff., sowie (1975), S. 617 ff.

Im Niskanen Modell handelt es sich ursprünglich um die Bereitstellung eines öffentlichen Gutes.

24 Vgl. Niskanen (1971), S. 38.
} 
von Leistungserfullung und Effizienzsteigerung ist, geht es ihnen vor allem um öffentliche Reputation, den Erhalt von Vergünstigungen bzw. Sonderzulagen, Entscheidungsmacht und möglichst viel Autonomie. Alle Ziele bis auf das letzte sind positiv und monoton von der Budgetgröße abhängig. ${ }^{25}$ Der Prinzipal stellt der Behörde ein Budget zur Verfügung. Dabei produziert eine Behörde mit dem Budget keine Anzahl an Outputs, dafür aber ein Niveau an Aktivitäten, das mehr oder weniger ein Wettbewerbsklima in der Telekommunikationsbranche schaffen soll. ${ }^{26}$ Für die Behörde fallen in diesem Zusammenhang Kosten an. Aufgrund der asymmetrischen Informationsverteilung hat der Prinzipal keinen Einblick in die Kosten der Behörde. Der Prinzipal kennt nur den Ertragsplan und das Budget und kann deshalb nicht bestimmen, ob das bereitgestellte Outputniveau pareto- effizient ist oder nicht. Diese asymmetrische Informationsverteilung befreit die Behörde unter der Annahme, daß die Behörde kein Geld an den Prinzipal zurluckzahlen muß, davon, ihr Budget unter der Einschränkung der Deckung der Produktionskosten zu maximieren. ${ }^{27}$ D.h. es gibt Anreize, das Budget immer vollständig auszugeben, anstatt die Nettoerträge des Prinzipals zu maximieren. ${ }^{28}$ Dadurch bindet die Behörde Gelder an sich, die der Prinzipal sonst anderweitig verwenden könnte. Weil die Gehälter der Mitglieder einer Behörde unabhängig von einer Effizienzsteigerung sind, folgt daraus, daß sie keine finanziellen Anreize haben, ihre Effizienz zu erhöhen.

Aufgrund der asymmetrischen Informationsverteilung zwischen Agentur und Prinzipal kann die Regierung im Sinn von ,hidden information' und ,hidden action' sowohl die Qualität der Leistung der Regulierungsbehörde als auch die erhaltenen Informationen nicht beurteilen. Dies ermöglicht zum einen gemäß ihrem eigenen Interesse in bezug auf den Selbsterhalt zu handeln und zum anderen gewährt es Interessengruppen, Einfluß auf die Entscheidungsfindung der Agentur zu ihren Gunsten zu nehmen. ${ }^{29}$

Vgl. Mueller (1989), S. 252.

Beispielsweise kann die Arbeit der Polizei nicht in Outputeinheiten an innerer Sicherheit gemessen werden.

Die Budgetmaximierung kann unter zwei Gesichtspunkten erfolgen. 1. Budgetmaximierung unter der Annahme, daß die Kosten nicht das Budget uberschreiten dürfen (,budget constrained '). 2. Budgetmaximierung unter der Annahme, daß die Nachfrageseite beschränkend wirkt (,demand constrained').

Dies erfolgt zum Nachteil der Konsumenten. Die Konsumentenrenten aus der Produktion eines Outputniveaus der Behörde werden von den zusätzlichen Kosten, die die Behörde verursacht, die nicht mit Grenzerträgen gedeckt werden kőnnen, aufgebraucht. Vgl. Mueller (1989), S. 253 f.

Vgl. dazu Kap. 6.2.3. 


\subsubsection{VERHALTENSKALKÜL DES AgENTEN}

Der Agent schließt einen Vertrag mit der Agentur über die zu erbringende Leistung. In diesem Zusammenhang beinhaltet der Vertrag beispielsweise eine kostenorientierte Bereitstellung eines Zugangs zu seiner Netzinfrastruktur. Dies umfaßt auch die Zusammenschaltung. D.h. der Vertrag umfaßt nicht nur die Öffnung des Netzes für Wettbewerber, sondern gibt auch noch den Preis vor, zu dem die Netzinfrastruktur bereitgestellt werden soll. ${ }^{30}$ Hier befindet sich ein entscheidender Unterschied zur herkömmlichen Prinzipal-Agent- Theorie, in der im allgemeinen die Verträge beidseitig freiwillig eingegangen werden. In dem gegebenen Regulierungskontext verfügt der Agent über keine Entscheidungsfreiheit sowohl über die Vertragsschließung als auch über die Vertragsgestaltung. Er ist gesetzlich zu der Vertragserfüllung gezwungen und kann nicht jederzeit aus dem Vertrag wieder aussteigen. Außerdem wird mit dem Vertrag nicht das unternehmerische Risiko auf beide Vertragspartner aufgeteilt, sondern lastet weiterhin allein auf den Schultern des Agenten. Der Agent unterliegt wie jeder andere Akteur dem Selbsterhaltungstrieb, nämlich seinen unternehmerischen Gewinn zu maximieren und langfristig seine Existenz zu sichern. D.h. die von ihm zu erbringende Leistung steht in einem grundsätzlichen Konflikt zu seinen eigenen Interessen, weil eine kostenorientierte Bereitstellung seines Netzes bedeutet, daß er keinen Gewinn macht und vielleicht sogar nicht einmal seine Gesamtkosten inklusive seiner getätigten irreversiblen Investitionskosten in die Netzinfrastruktur decken kann. Aus diesem Grund wird der Agent prinzipiell ein Interesse daran haben, seine Vorteile bedingt durch eine asymmetrische Informationsverteilung auszuschöpfen. Er wird also nicht seine wahren anfallenden Kosten nennen, sondern einen Anreiz haben, sie möglichst hoch auszuweisen. Zudem wird er alle Möglichkeiten ausschöpfen, Einfluß auf die Regulierungsbehörde zu nehmen.

\subsubsection{EINFLUB VON INTERESSENGRUPPEN}

Die Unterstellung eines benevolenten Regulators in der klassischen Regulierungstheorie ist schon in Kap. 6.2.1 hinterfragt worden, in dem die Eigeninteressen einer Behörde dargestellt worden sind. In diesem Kapitel soll es nun darum gehen, daß die Behörde zudem von außen in ihren Entscheidungen beeinflußbar ist.

Eine Deregulierung durch regulierende Eingriffe umzusetzen, verfolgt das Ziel, Monopolrenten auf Konsumenten umzuverteilen. Dabei muß berücksichtigt werden, daß es immer Interessengruppen geben wird, die auf die Umverteilung zu ih-

$30 \quad$ Vgl. dazu Kap. 2.4.2 und 2.4.3. 
ren Gunsten auf die Agentur Einfluß nehmen wollen. ${ }^{31}$ Interessengruppen sollen hier jene Gruppen umfassen, die von den Entscheidungen der Behörde unmittelbar betroffen sind. $\mathrm{Zu}$ ihnen zählen neben dem regulierten Unternehmen ebenso die Wettbewerber. Die Wettbewerber des Incumbents treten als eine Interessengruppe gegenüber dem Agenten und der Agentur auf. Ihre Ziele sind, möglichst billig in den Markt einzusteigen und nach Markteintritt möglichst geschützt zu sein. Das regulierte Unternehmen will ebenso seine Interessen von der Regulierungsbehörde berücksichtigt sehen. Dabei besteht ein grundsätzlicher Interessenkonflikt zwischen Wettbewerber und Incumbent. Beide Akteure werden Einfluß auf die Behörde nehmen wollen, damit sie jeweils zu den eigenen Gunsten entscheidet bzw. um sie gegen den anderen zu instrumentalisieren. Hier soll auf zwei Möglichkeiten der Einflußnahme eingegangen werden: Zum einen können Interessengruppen die Agentur mit monetären Seitenzahlungen bestechen, um in den regulierungspolitischen Entscheidungen begünstigt zu werden. D.h. eine Firma zahlt Bestechungsgelder an die Agentur, damit sie die Entscheidungen zu ihren Gunsten trifft. Dabei sei bemerkt, daß das für Seitenzahlungen bereitgestellte Geld nicht vollständig für die Bestechung ausgegeben werden kann, weil die daraus entstehenden Organisationskosten ebenso bezahlt werden müssen. ${ }^{32}$ Zum anderen gelingt den Interessengruppen eine Einflußnahme aufgrund von personellen Verflechtungen. Die persönlichen Verbindungen zwischen Angestellten der Regulierungsbehörde und Angestellten in der Industrie sollten nicht unterschätzt werden. Ursache dafür ist, daß es nur einen kleinen Kreis an fachlich qualifiziertem Personal gibt, das konkret auf die vorliegende Problematik, nämlich Einführung von Wettbewerb auf netzinfrastrukturbasierten Märkten, spezialisiert ist und das sich persönlich untereinander kennt. Aus diesem Expertenpool bedienen sich sowohl die Regulierungsbehörde als auch Incumbent und Wettbewerber zur Besetzung der jeweiligen Schlusselpositionen und werben sie sich gegenseitig ab.

Seitenverträge z.B. in Form von geheimen Absprachen zwischen Agentur und Interessengruppen können sowohl über monetäre Seitenzahlungen als auch über persönliche Verbundenheit zustande kommen. ${ }^{33}$ Die Folgen von Einflußnahme auf Regulierungsentscheidungen der Behörde in Form von Seitenverträgen können sein, daß die Marktstruktur und das Marktverhalten aller Anbieter verzerrt wird. Zum einen kann es zu einer Förderung nicht wettbewerbsfähiger Unterneh-

31 Vgl. Crew/Kleindorfer (2002), S. 9.

32 Zur Ineffizienz monetärer Transfers vgl. Laffont/Tirole (1994a), S. 477 und S. 489. Organisationskosten sind für eine kleine Gruppe mit hohem Kapitalstock kleiner als für eine große Gruppe ohne Kapitalstock wie die aller Steuerzahler. Vgl. Olson (1965), Laffont/Tirole (1994a), S. 477 f.

33 Solche Seitenverträge können stabil sein, wenn beide Seiten an ihrem Versprechen zu kooperieren, festhalten, weil sie: 1. ihr Ehrenwort halten, 2. dadurch Reputation erlangen und 3. die Benefits dadurch anwachsen. Vgl. Laffont/Tirole (1994a), S.478. 
men kommen, die zu produktiver Ineffizienz führen würde. Zum anderen kann die Wettbewerbsintensität der einzelnen Unternehmen sinken, was allokative wie produktive Ineffizienz hervorriefe. Seitenverträge lassen sich dann verhindern, wenn die Kosten für das Abschließen eines Seitenvertrages für beide vertragschließenden Parteien höher sind als der Nutzen daraus.

\subsection{StRategische INTERAKTIONEN ZWISChEN PRINZIPAL, AgENTUR UND AGENT IN DER TELEKOMMUNIKATION}

Die Erkenntnisse aus der Prinzipal-Agent- Theorie sind in die Theorie der Regulierungsökonomie durchaus schon eingeflossen. ${ }^{34}$ Die praktizierte Regulierungspolitik, wie sie in Kapitel 5.4 dargelegt wurde, hat allerdings die Erkenntnisse aus der Prinzipal-Agent- Theorie nicht gebührend berücksichtigt bzw. die Ansätze sind ebenso wenig praktikabel wie traditionelle Regulierungsansätze. Die Konsequenzen daraus sollen anhand dieses Kapitels belegt werden. Es finden nämlich strategische Interaktionen zwischen den einzelnen Akteuren statt, die belegen, daß viele Entscheidungen das Ergebnis politökonomischer Einflüsse sind und nicht auf theoretischen ökonomischen Grundlagen beruhen. Im folgenden sollen die Ziele der einzelnen Akteure kurz vorgestellt werden, um dann ihre jeweiligen ,strategischen Spiele' zu skizzieren, mit denen sie versuchen, ihrem Ziel näher zu kommen.

\subsubsection{SPIELE DES AGENTEN}

Das Ziel des Incumbents ist, bei der Liberalisierung möglichst wenig Marktanteil $\mathrm{zu}$ verlieren und seine marktbeherrschende Stellung zu behalten. Aus diesem Grund wird er mit Hilfe strategischer Markteintrittsbarrieren versuchen, Wettbewerbern den Markteintritt möglichst teuer bzw. schwierig zu gestalten, um gegebenenfalls ihren Markteintritt $\mathrm{zu}$ verhindern. ${ }^{35}$ Hierbei geht es insbesondere um

34 Vgl. dazu Laffont/Tirole (1994a), die verschiedene Regulierungsdesigns unter Berücksichtigung unterschiedlicher Anreizproblematiken zusammengefaßt haben. Dabei wird diesen Regulierungsansätzen vorgeworfen, nur sehr begrenzt in der Praxis anwendungsfahig zu sein, weil davon ausgegangen wird, daß eine Regulierungsbehörde zu vielen keinen Informationen keinen Zugang hat. Vgl. Crew/Kleindorfer (2002), S.11. Crew/Kleindorfer (2002), S. 14 schlagen ein Price-Cap- Verfahren vor, weil es die genauesten Aussagen triff, ohne daß der Regulator über viel Informationen verfügen muß.

3s Eine Verdrängungsstrategie seitens des Incumbents mit Hilfe von Preisdumping kann rational sein. Vgl. Bolton u.a. (2000). Insbesondere dann, wenn Finanzmärkte nicht funktionieren und 
den Zugang zu seiner Netzinfrastruktur. Mit zwei Beispielen aus dem Teilnehmeranschlußbereich kann diese These untermauert werden, nämlich dem Streit über den Zugang zur Letzten Meile sowie um die DSL- Anschlüsse.

Der Zugang zur Letzten Meile wird uber den Teilnehmeranschlußleitungsvertrag geregelt. $\mathrm{Da}$ der Incumbent in weiten Teilen der deutschen Netzinfrastruktur der einziger Anbieter von Zugängen zur Letzten Meile ist, gibt es für ihn hohe Anreize, den Zugang zu TVSt bei der Vermietung der entbündelten Teilnehmeranschlüsse als strategische Markteintrittsbarriere zu mißbrauchen. Der Zugang zur Teilnehmeranschlußleitung stellt einen fortwährenden Konflikt zwischen Incumbent und Regulierungsbehörde dar. Dem Incumbent wird immer wieder vorgeworfen, Markteintritt durch fehlende verbindliche Vereinbarungen und $\mathrm{zu}$ lange Bereitstellungsfristen $\mathrm{zu}$ behindern. Vertragsbedingungen $\mathrm{zu}$ dem Teilnehmeranschlußleitungsvertrag ist derzeit Gegenstand eines entsprechenden Regulierungsverfahrens. ${ }^{36}$

In bezug auf die T-DSL-Anschlüsse (für besonders leistungsfähige Internetzugänge) hat die DTAG im Jahr 2001 mit technischen Begründungen den Markteintritt von Wettbewerbern auf der Letzten Meile verzögert und damit verhindert. In diesem Zusammenhang lag ein Verfahren bei der RegTP wegen Wettbewerbsverhinderung vor. Der Incumbent hat inzwischen eine 8 Wochen Frist für die Ermöglichung eines DSL-Anschlusses in einer OVSt einzuhalten. ${ }^{37}$

\subsubsection{SPIELE DES WETTBEWERBERS}

Die Wettbewerber treten gemeinsam als Interessengruppe in Form des, Verbands der Anbieter von Telekommunikations- und Mehrwertdiensten' (VATM) auf. ${ }^{38}$

die Wettbewerber schlechtere Finanzierungsmöglichkeiten haben als der ehemalige Monopolist. Vgl. Bolton/Scharfstein (1990).

36 Vgl. RegTP (2001b), S. 106, Pressemitteilung der RegTP vom 12.02.2002. Zudem sind die Bereitstellungsentgelte und Kündigungsentgelte furr den Zugang zum Teilnehmeranschluß der DTAG nach ausfuhrlicher Kostenanalyse um bis zu $24 \%$ gesenkt worden. Vgl. dazu Pressemitteilung der RegTP vom 12.04.2002.

Im Dezember 2001 wurde seitens der RegTP ein T-DSL- Entgeltregulierungsverfahren gegen die DTAG vom 30.03.2001 wiederaufgenommen. Damals schritt die RegTP gegen die festgestellten teilweise nicht kostendeckenden T-DSL-Preise nicht ein, weil eine Verdrängungswirkung derzeit nicht erwiesen war. Im Januar 2002 ist das Verfahren wieder eingestellt worden, weil die DTAG ein neues Entgeltmodell festgelegt hat, bei dem es zu deutlichen Preiserhöhungen bei den monatlichen Überlassungsentgelten und den einmaligen Bereitstellungspreisen kommt. Vgl. Pressemitteilung der RegTP vom 22.01. 2002.

38 Der VATM umfaßt mehr als 50 im deutschen Markt operativ tätigen Telekommunikationsund Multimediaunternehmen und reprăsentiert ca. $80 \%$ des von den privaten Anbietern erzielten Telekommunikations-Gesamtumsatzes. Vgl. http://www.vatm.de. 
Der VATM ist das Sprachrohr für die Anliegen und Forderungen von bereits etablierten Wettbewerbsunternehmen, indem er die gemeinsamen Interessen seiner Mitgliedsunternehmen gegenüber der Öffentlichkeit darstellt. Das Ziel des Verbandes ist es, Wettbewerbsbedingungen zu schaffen, die allen Unternehmen Planungssicherheit und Investitionssicherheit geben. In der Regel sind weitestgehend alle Mitgliedsunternehmen bei der Bereitstellung von Diensten auf die Netzinfrastruktur des Incumbents angewiesen. Deswegen käme es ihnen gelegen, einen möglichst einfachen und preiswerten Zugang zur Netzinfrastruktur zu haben und dann möglichst geschützt im Markt zu sein. Dies betrifft die Regelung von

- Zugang und Zugangsentgelten zur Netzinfrastruktur auf der Weitverkehrsebene wie auf der regionalen sowie Teilnehmeranschlußebene,

- Zusammenschaltungsleistungen sowie ihre Entgeltbestimmung,

- Zugangsbedingungen sowie Entgeltbestimmungen von Mietleitungen ${ }^{39}$.

Eine Entgeltregulierung von Netzzugängen betrifft nur Unternehmen mit marktbeherrschender Stellung. Dabei ist eine Regulierung an den Kosten der effizienten Leistungsbereitstellung zum Vorteil der Wettbewerber, die nicht über eigene Netzinfrastruktur verfügen. ${ }^{40}$ Es liegt im Interesse der Wettbewerber, wenn sich die Behörde bei der Bestimmung von Entgelten möglichst an den Grenzkosten bzw. Inkrementalkosten orientiert. Wittern die Wettbewerber einen Marktmißbrauch seitens des Incumbents, rufen sie die RegTP an. In bezug auf die Anordnung von Netzzusammenschaltungsleistungen verhandeln die Wettbewerber gar nicht erst, sondern schalten gleich die Regulierungsbehörde ein. ${ }^{41}$

Die Interessengruppe versucht nach außen, Einfluß auf die Behörde zu gewinnen, um bei regulierungspolitischen Entscheidungen begünstigt zu werden. $\mathrm{Zu}$ diesem Zweck formuliert sie nach außen Forderungen an die Regulierungsbehörde und an die Regierung und kommentiert marktrelevante Entscheidungen aus Sicht der Mitgliedsunternehmen. Nach innen leitet der Verband alle verfügbaren Informationen über aktuelle und zukünftige regulatorische Entwicklungen an alle Mitglieder weiter.

Innerhalb der Interessengruppe muß berücksichtigt werden, daß alle Mitgliedsunternehmen im Grunde im Wettbewerb zueinander stehen. Es entstehen zunehmend divergierende Unternehmensinteressen, weil die einzelnen Wettbewerber sich unternehmensstrategisch unterschiedlich auf die Heterogenisierung des Marktes ausrichten. Einige Unternehmen bleiben bei der herkömmlichen Strategie, Dienste weitestgehend auf dem angemieteten Netz des Incumbents anzumieten. Dann ha-

39 Zu den neuen Rahmenbedingungen im Mietleitungsmarkt vgl. Pressemitteilung der RegTP vom. 03.06. 2002. Dort werden Bereitstellungszeiten sowie das Einhalten von Lieferzeiten geregelt.

40 Vgl. Kap. 2.4.2 und 5.4.

41 Vgl. Kap.2.4.3. 
ben sie weiterhin Interesse an einem möglichst preiswerten Zugang zum Netz des Incumbents. Andere Unternehmen bauen dagegen zunehmend eine Netzinfrastruktur auf. Dann verfolgen sie das Interesse, daß das Zugangsentgelt ihre hohen risikobehafteten irreversiblen Investitionskosten deckt. Aus diesem Grund kann es über kurz oder lang zu einem fundamentalen Interessenkonflikt innerhalb des Verbandes kommen, so daß irgendwann der Verband nicht mehr nach außen mit einer Stimme für alle seine Mitglieder sprechen kann.

\subsubsection{SPIELE DER AGENTUR}

Das Ziel der Regulierungsbehörde ist zum einen gemäß der Annahme über die Eigennutzorientiertheit, möglichst lang ihre Existenz zu sichern und auszubauen. Zum zweiten braucht sie kurzfristig sichtbare Erfolge, die sie dem Prinzipal unterbreiten und damit eine Reputation in der Öffentlichkeit erwirken kann. Mit anderen Worten geht es der Behörde zwar darum, kurzfristig möglichst viele Wettbewerber $\mathrm{zu}$ verzeichnen, die Dienste bereitstellen. Die Markteintrittsschwelle hält sie für solche reinen Diensteanbieter möglichst gering, indem sie ein möglichst niedriges Zugangsentgelt festlegt. $\mathrm{Da} \beta$ sich die Regulierungsbehörde auf TELRIC bei Zugangsentgelten beruft, zeigt, daß sie eine kurzfristige Perspektive vor Augen hat. Der Markteintritt reiner Diensteanbieter und folglich der Dienstewettbewerb wird mit einer Regulierung der Zugangsentgelte auf der Basis von TELRIC ganz bestimmt gefördert. Allerdings bleibt die Existenz reiner Diensteanbieter im besonderen Maße vom marktbeherrschenden Unternehmen abhängig, das ihnen den Zugang zur Netzinfrastruktur zur Verfügung stellen muß. Da dies immer ein Herd bedeutsamen Konfliktpotentials darstellt, wird sich das Bestehen einer Regulierungsbehörde immer legitimieren lassen. Man kann der Regulierungsbehörde folglich unterstellen, daß ihre regulatorischen Eingriffe keine Langfristperspektive aufweisen. Denn ein niedriges festgelegtes Zugangsentgelt schafft weder beim regulierten Unternehmen noch insbesondere bei den Wettbewerbern Anreize für Investitionen in die Netzinfrastruktur. Damit findet eine Behinderung des infrastrukturbasierten Wettbewerbs statt, der als einziger langfristig einen Wettbewerb und damit auch niedrige Preise für den Kunden garantieren kann. Solange es aber den nicht gibt, wird sich immer die Existenz einer Regulierungsbehörde rechtfertigen lassen.

Bei der Festlegung der Zugangs- und Zusammenschaltungsentgelte auf Basis von TELRIC bzw. analytischen Kostenmodellen ist es ohnehin fraglich, wie weit der Interpretationsspielraum der Behörde in bezug auf das Ergebnis ausgeschöpft wird. Beispielsweise hat die Regulierungsbehörde in Deutschland auf der Grundlage der Kosten der effizienten Leistungsbereitstellung nach dem analytischen 
Kostenmodell vom WIK den monatlichen Mietpreis eines Teilnehmeranschlusses auf einer herkömmlichen Kupferdoppelader für 2001 bis 2003 auf 24,40 DM festgelegt. Der Incumbent hatte ein höheres Entgelt für das monatliche Anmieten eines Teilnehmeranschlusses vorgeschlagen $\left(34,03 \mathrm{DM} /\right.$ monatlich $\left.^{42}\right)$, die Wettbewerber haben dagegen anhand von Studien ebenso auf der Grundlage der Kosten für die effiziente Leistungsbereitstellung ein niedrigeres Entgelt (15-17,80 $\mathrm{DM} /$ monatlich $^{43}$ ) ermittelt. Die Regulierungsbehörde hat also einen Preis festgelegt, der ungefähr in der Mitte der beiden Forderungen war. Es ist anzunehmen, daß Ergebnisse auf der Grundlage von Kostenanalysen einen Richtungsmaßstab darstellen. Die Entscheidung ist letztendlich zusätzlich von anderen Faktoren abhängig. Es ist vermutlich bewußt die Entgelthöhe zwischen den Forderungen gewählt worden, um keine Partei vor den Kopf zu stoßen.

Ein anderer Diskussionspunkt ist, daß im Grunde seit der Liberalisierung des Telekommunikationsmarktes die monatliche Grundgebühr, die der Incumbent vom Endkunden verlangt, bislang viel zu niedrig war, weil sie nicht die wahren Kosten widerspiegelte und aufgrund von Quersubventionierungen finanziert worden ist. Weder die Regulierungsbehörde noch der Incumbent oder der Wettbewerber haben etwas an dieser Situation geändert, weil eine Forderung nach Erhöhung einen bedeutsamen Verlust an Publizität bedeutet hätte. Die Regulierungsbehörde hätte sich sowohl bei der Regierung als auch bei der Bevölkerung sehr unbeliebt gemacht. Vordergründig hätten sie verantworten müssen, daß Wettbewerb zu Preiserhöhungen zum Schaden der Konsumenten führt. Der Incumbent, der ebenso schlechte Schlagzeilen fürchtet, wollte sich bislang keinen zusätzlichen Publizitätsverlust leisten. Die Wettbewerber hatten ebenso kein Interesse an einer Grundgebührerhebung, da sie letztendlich auf eine Senkung der Preise für die Anmietung und Bereitstellung der Leitungen abzielen. Diese differieren geringfügig nach oben hin von der Grundgebühr. Die Grundgebühr gab bislang den Orientierungswert nach unten vor. Inzwischen ist der Bann gebrochen, und die DTAG hat zum 1. Mai 2002 die Grundgebühr für Telefonanschlüsse um ca. $5 \%$ erhöhen dürfen (für ISDN-Anschlüsse um 3,2\%).

\subsubsection{FAZIT UND BEWERTUNG}

Die Etablierung wirksamen Wettbewerbs ist letztendlich abhängig vom

- Engagement der Regulierungsbehörde, die Aufgabe umzusetzen,

\footnotetext{
$42 \quad$ Vgl. RegTP (2001b), S. 79.

43 Vgl. VATM (2001), S. 14.
} 
- Engagement des regulierten Unternehmens, mit der Agentur zusammenzuarbeiten,

- Erfolg der Einflußnahmen der Interessengruppen auf die Behörde,

- Zufall bzw. Umweltzustand.

Es stellt sich die Frage, inwiefern die Existenz einer sektorspezifischen Regulierungsbehörde bei der Etablierung von Wettbewerb sinnvoll ist oder ob diese Aufgabe nicht ebenso dem Kartellamt ubbertragen werden kann.

Eine sektorspezifische Regulierungsbehörde bringt zwar ein hohes Niveau an telekommunikationsspezifischer Fachkompetenz mit. Dagegen spräche beispielsweise für eine Verlagerung des Aufgabengebietes zum Kartellamt, daß es eine Behörde weniger găbe, die mit dem Ziel der Budgetmaximierung und möglichst langer Lebensdauer letztendlich den Konsumenten als Steuerzahler schadet, da sie öffentliche Gelder an sich bindet. Außerdem wäre eine Einflußnahme von Interessengruppen, bedingt durch den gemeinsamen Personalpool, nicht in dem Maße gegeben, wie es bei einer sektorspezifischen Behörde der Fall ist, da die personellen Überschneidungen beim Kartellamt geringer wären.

Abschließend seien noch wenige Worte dazu gesagt, inwiefern die Anwendung des Principal-Agent- Ansatzes zur Beurteilung von regulierungsökonomischen Fragestellungen problematisch ist: Ein zentraler Gesichtspunkt ist, daß bei privaten Transaktionen der Prinzipal die Kosten bei Irrtümern trägt. Liegt dagegen der Regulator falsch mit seinen Annahmen uber den Agenten, dann müssen entweder die Konsumenten oder das regulierte Unternehmen die Konsequenzen tragen ${ }^{44}$. Die Antizipation dieser Konsequenzen wird zu strategischen Interaktionen fuihren, die wiederum Effekte auf die ,common knowledge' Annahmen ${ }^{45}$ haben sowie auf die Ergebnisse von Regulierung. Eine ăhnliche Problematik liegt bei der Risikoverteilung vor. In der traditionellen Prinzipal-Agent- Theorie wird mit dem Abschluß des Vertrages das Risiko bedingt durch eine unsichere Umwelt auf beide Vertragsparteien verteilt. Der in der Regulierungsdebatte abzuschließende Vertrag zwischen dem $\mathrm{zu}$ regulierenden Unternehmen und der Regulierungsbehörde beläßt weiterhin das gesamte unternehmerische Risiko beim Agenten. Ein weiterer Aspekt ist, daß in der traditionellen Prinzipal-Agent- Theorie der Agent frei ist zu entscheiden, ob er einen Vertrag mit dem Prinzipal eingehen möchte oder nicht. Angewendet auf einen Regulierungszusammenhang steht es dem zu regulierenden Unternehmen nicht frei, mit der Regulierungsbehörde zusammenzuarbeiten oder nicht, sondern er wird gesetzlich dazu gezwungen.

44 Vgl. Crew/Kleindorfer (2002), S. 11.

45 Die ,common knowledge' Annahme besagt, daß das regulierte Unternehmen seine Schlüsselparameter in dem Wissen wăhit, daß die Parameter des Regulator auf denen des Unternehmens basieren. 
Sarah Eliza Schniewindt - 978-3-631-75130-5 Downloaded from PubFactory at 01/11/2019 07:57:00AM via free access 
Ziel dieser Arbeit war eine Analyse der verschiedenen Möglichkeiten zur Einführung von Wettbewerb im Teilnehmeranschlußbereich der Telekommunikation. Die Ergebnisse werden im folgenden noch einmal zusammengefaßt:

\section{Verlegung, doppelter' Infrastruktur}

Da der Incumbent weitestgehend die Teilnehmeranschlußbereiche in Deutschland monopolisiert, hat ein Wettbewerber die Möglichkeit, eine eigene Netzinfrastruktur neben der des Incumbents zu legen. Dies ist im allgemeinen wegen der hohen versunkenen Kosten und der sich daraus ergebenden Kostensubadditivität ökonomisch ineffizient, es sei denn, die exogenen Parameter, nämlich die Nachfrage oder der technische Fortschritt, ändern sich. Da die Entwicklungen in der Telekommunikation insbesondere in den letzten Jahren von rasanten technologischen Veränderungen und damit einhergehender steigender Nachfrage geprägt sind, hat eine ausführliche Analyse gezeigt, daß das Verlegen einer zweiten Infrastruktur für einen Wettbewerber ökonomisch sinnvoll sein kann.

\section{Substitutiver Wettbewerb}

Die zweite Möglichkeit der Einführung von Wettbewerb auf der Letzten Meile bestünde in Markteintritten von Unternehmen, die mit Hilfe substitutiver Technologien Telekommunikationsdienste anbieten. In Frage kämen Umrüstungen bereits bestehender Infrastrukturnetze wie Fernseh- oder Stromkabel sowie das Internet oder der Neuaufbau einer Netzinfrastruktur, die wegen einer anderen Technologie nicht die vergleichbar hohen versunkenen Kosten hătte, wie z.B. funkgestützte Anschlußtechnologien. Es ist eine ausführliche Marktabgrenzung vorgenommen worden, um das Wettbewerbspotential dieser alternativen Anschlußtechnologien auszuleuchten. Das Fazit aus der Analyse ist, daß die untersuchten substitutiven Anbieter als potentielle Wettbewerber in Frage kommen. Ob sich ein Markteintritt im Hinblick auf Gewinn- und Erfolgschancen lohnt, muß ein potentieller Anbieter mit seinem unternehmerischen Risiko abwägen.

\section{Anmieten von Teilnehmeranschlüssen}

Die dritte Möglichkeit, Wettbewerb auf der Letzten Meile zu fördern, besteht darin, Wettbewerbern das Anmieten von Teilnehmeranschlüssen des Incumbents zu ermöglichen. Die. Form des Markteintritts erleichtert insbesondere den Wettbewerbern auf den nachgelagerten Märkten den notwendigen Netzzugang und stärkt damit implizit den Dienstewettbewerb. Sie stellt im Vergleich zu den ersten beiden Alternativen eine Variante dar, die in kürzester Zeit zu vielen Marktein- 
tritten auf den nachgelagerten Märkten mit der Folge eines raschen Preisverfalls führt. Aufgrund der Gefahr des Mißbrauchs des Netzzugangs als Markteintrittsbarriere zieht das Anmieten von Teilnehmeranschlüssen eine detaillierte Zugangsregelung einschließlich einer Regelung der Zugangsentgelte nach sich. Im Rahmen dieser Arbeit sind verschiedene Preisregeln für die Bestimmung von $\mathrm{Zu}-$ gangsentgelten vorgestellt worden. Dabei ging aus dem Vergleich der Preisregeln die ECPR als diejenige hervor, die produktive Effizienz erzielt, d.h. die den Markteintritt effizienter Unternehmen unterstützt und gleichzeitig ertragsneutral für den Incumbent ist. Daraus folgt zudem eine Förderung infrastrukturbasierten Wettbewerbs.

In der Regulierungspraxis orientiert man sich in Deutschland dagegen entsprechend der gesetzlichen Vorgabe an den Kosten der effizienten Leistungsbereitstellung. Als Preisregel werden die TELRIC mit einem Gemeinkostenaufschlag zugrunde gelegt. Ein auf diesem Weg ermitteltes Zugangsentgelt deckt nicht die Opportunitätskosten und nicht die irreversiblen Investitionskosten des Incumbents. Deswegen bringt es folgende Probleme mit sich: Da der Incumbent erstens nicht kostendeckend Zugang zu seinem Netz gewähren muß, wird der Anreiz, in seine eigene Netzinfrastruktur zu investieren, gesenkt. Außerdem schafft eine TELRIC Preisregel ebenso keine Anreize für Wettbewerber, in die Infrastruktur zu investieren. Daraus folgt, daß ihre Abhängigkeit vom Incumbent bestehen bleibt. Drittens räumt diese Preisregel der Regulierungsbehörde eine SchlüsselPosition ein, die durch die Abhängigkeit der Wettbewerber auf dem nachgelagerten Markt vom Incumbent bedingt ist. Auf diesem Wege legitimiert die Regulierungsbehörde dauerhaft ihre Existenz und verhindert so weiterhin die Etablierung von Wettbewerb.

Der physische Zusammenbruch des amerikanischen Strommarktes in den Jahren 2000/2001 hat gezeigt, welche desaströsen Folgen eine halbherzige Liberalisierung von öffentlichen Monopolen haben kann. Kernursache dafür war eine Preisfixierung, die zu mangelnden Investitionsanreizen geführt hat, die letztendlich das marktliche Versagen ausgelöst haben.

Auch der Telekommunikationsmarkt ist wegen der Entgeltvorgaben für Zugangsgewährung und Zusammenschaltung durch Preisfixierungen gekennzeichnet. Als Folge dessen ist ein europaweites Stagnieren des Investitionsvolumens seit der Liberalisierung zu verzeichnen. Dies muß unbedingt als bedrohliches Vorzeichen interpretiert werden.

Die Einführung von Wettbewerb in netzinfrastrukturbasierten Sektoren ist eine äußerst diffizile Angelegenheit. Ihr Erfolg darf nicht an möglichst schnellen und vielen Markteintritten sowie möglichst schnellen und hohen Preissenkungen gemessen werden. Wirksamer Wettbewerb muß die Chance bekommen, wachsen 
und gedeihen zu dürfen. Als guten Nährboden im Sinne von ökonomischen Rahmenbedingungen braucht Wettbewerb die Beseitigung institutioneller Markteintrittsbarrieren, die Pflicht zur Gewährung von Netzzugang und Zusammenschaltung und Nummernportabilität. Aber vor allem braucht er viel Zeit, sich entfalten zu können.

Zudem sei angemerkt, daß die Gewährleistung der ökonomischen Rahmenbedingungen nicht die Existenz einer sektorspezifischen Regulierungsbehörde legitimiert, da diese Aufgabe ebenso von einer allgemeinen Wettbewerbsbehörde ausgefuhrt werden kann. Der Gefahr geheimer Preisabsprachen nach der Etablierung des Wettbewerbs auf der Letzten Meile kann ebenfalls mit einer allgemeinen Wettbewerbsbehörde begegnet werden. Hierbei muß berlicksichtigt werden, daß aus dem möglichen Bestehen geheimer Preisabsprachen keine prinzipielle Notwendigkeit regulierender Eingriffe resultiert, weil die Stabilität solcher Absprachen von dem Produktdifferenzierungsgrad abhängt. Ein Eingriff seitens einer regulierenden Institution kann für die Wettbewerber wie auch für die Konsumenten unvorteilhafter sein als eine instabile Preisabsprache.

Bei Eingriffen seitens der sektorspezifischen Regulierungsbehörde in das Marktgeschehen sollte außerdem bedacht werden, daß es sich hierbei nicht um eine neutrale Instanz handelt, sondern um einen Akteur im wirtschaftspolitischen Geschehen, der einen Eigennutz verfolgt und von Interessengruppen beeinflußbar ist. 
Sarah Eliza Schniewindt - 978-3-631-75130-5 Downloaded from PubFactory at 01/11/2019 07:57:00AM via free access 


\section{ANHANG}

\section{A.1 DIE ATM- TECHNIK}

Ausgangslage der Breitbandentwicklung sind die schon erwähnten Netze und Dienste wie das Schmalband-ISDN (64 kbit/s für Grunddienste, $140 \mathrm{Mbit} / \mathrm{s}$ für Mehrwertdienste, z. B. Videokonferenztechniken und Verteildienste), das Datex-P Netz (für Datenübertragungen bis $64 \mathrm{kbit} / \mathrm{s}$ ) und verbindungslose Netz- und Transportdienste (im LAN- und MAN- Bereich mit Datenübertragungsraten von 2-34 Mbit/s). Diese Übertragungs- und Dienstetechniken haben gemeinsam, daß sie während einer Übertragung konstante Bitraten transportieren. Wechseln sich die Dienste während einer Verbindung ab, werden während der Übertragung unterschiedliche Bitraten benötigt (z.B. Bewegtbilder benötigen eine höhere Bitrate als Standbilder). Können nur konstante Bitraten übermittelt werden, muß für die Übertragung dementsprechend die höchste Bitrate, die für die angewendeten Dienste notwendig ist, gewählt werden. Es wird folglich eine Bitrate für den Zeitraum der Übertragung bereitgestellt, die unter Umständen für die gesamte Übertragungszeit gar nicht in Anspruch genommen werden muß. Daraus ergab sich das Forschungsvorhaben, eine Technik zu finden, die Übertragung von variablen $\mathrm{Bi}$ traten, die sich an dem jeweils eingesetzten Dienst orientieren, ermöglicht und möglichst hohe Übertragungsbandbreiten bereitstellt: die ATM- Technik.

Mit der ATM- Technik findet anstatt der herkömmlichen Leitungsvermittlung eine Paketvermittlung bei einer physikalischen Verbindung (verbindungsorientiert) statt. Die zu transportierende Information wird in gleich große Datenpakete bzw. Zellen (53 Byte) unterteilt. Jedes Paket wird mit der Zieladresse und der angemeldeten Bitrate des Empfängers versehen und in dieser Form verschickt. Es handelt sich um ein asynchrones Zeitmultiplexverfahren' mit konstanter Zellenlänge. ${ }^{2}$ ATM überträgt Zellen fester Länge. Jede Zelle hat die gleiche Größe, einen Zellenkopf und ein Informationsfeld. Im Zellenkopf befinden sich Informationen zur Steuerung der Zelle, im Informationsfeld die zu transportierende Nachricht. Das Übertragungsmedium ist in Zeitschlitzen (slots) organisiert, die frei mit Zellen belegt werden können. Die Belegung einer bestimmten Anzahl an ATM- Zellen innerhalb einer Beobachtungsdauer entspricht der bestimmten Bandbreite. Jede Kommunikationsquelle belegt Zellen entsprechend des augenblicklichen Bedarfs. Folglich wird die Übertragungstechnik unabhängig von den Übermittlungskanälen

Das ist eine Methode, bei der Digitalinformationen zeitlich verschachtelt auf einem Medium transportiert werden.

2 Ein Asynchroner Zeitmultiplex (ATD= Asynchronous Time Division) bedeutet, daß es keine feste zeitliche Zuordnung auf dem Übertragungsmedium gibt. 
der Kommunikationsquellen. Damit ist diese Technik geeignet, Signale mit festen und variablen Bitraten (d.h. verbindungsorientierte Datendienste) und auch paketorientierte Nachrichten (d.h. verbindungslose Datendienste) in einem Übertragungsmedium zeitgleich zu transportieren. Die maximale Übertragungskapazität ist dann erreicht, wenn alle Zeitschlitze durch Zellen besetzt sind. Die Transportbitrate beträgt $155 \mathrm{Mbit} / \mathrm{s}$ und kann in höheren Multiplexebenen der Synchron Digital Hierarchy (SDH) übertragen werden. Der Zellenstrom kann durch Multiplexund Vermittlungsvorgänge verändert werden. Die zeitliche Reihenfolge von Zellen einer gleichen Verbindung sind aber garantiert. Der zeitliche Abstand der Zellen einer Verbindung kann aber im Vergleich zur Ursprungssituation verändert werden (= Asynchronität). ATM ist für folgende Anwendungen verfügbar: ${ }^{3}$

- Im B-ISDN:

Die Einrichtung von ATM- VSt und andere ATM Einrichtungen bilden das BISDN Netz. Auf diese Weise bietet das öffentliche Telefonnetz Hochgeschwindigkeitsanschlüsse an, die die LANs miteinander verbinden, die MultimediaKommunikation unterstützen und standortübergreifende Privatnetze bilden können. Das heute bereits verfügbare MAN kann als Zubringernetz zum B-ISDN genutzt werden. Das Datex-P Netz kann im B-ISDN aufgehen.

- Im Backbone-Netz:

Die ATM Übertragungstechnik ist ideal als Backbone-Netz für die Vernetzung von LANs, weil sie über die hohe Übertragungsgeschwindigkeit verfügen und flexibel in den Bandbreiten sind.

- Im ATM-LAN:

Die ATM- Übertragungstechnik bildet gleichzeitig die Basis für die neueste LANGeneration. Die zukünftigen LANs werden ATM- Übertragungstechnik einsetzen und so zu Hochgeschwindigkeitsnetzen hochgerüstet. Momentan herrscht in den LANs ein Engpaßbereich, der hierdurch aufgehoben werden würde.

- In Endeinrichtungen:

ATM wird mit Hilfe von Adapterkarten in PCs und Workstations integriert werden und wird die interne Struktur dieser Endeinrichtungen beeinflussen können. Der Vorteil liegt in der Unterhaltung paralleler Verbindungen, wodurch sich eine erhebliche Leistungssteigerung ergibt.

- In Telekommunikations-Anlagen:

Die Erweiterung der ISDN Infrastruktur wird sich auch in Privatnetzen und Corporate Networks fortsetzen. ATM-TK Anlagen werden neben herkömmlichen Schnittstellen auch ATM-Schnittstellen zum öffentlichen Netz unterstützen. Auf diese Weise macht ATM die Bildung standortübergreifender Privatnetze möglich.

3 Vgl. Siegmund (1999), S. $815 \mathrm{ff}$. 
Die ATM-Technik zum Beispiel wird wie keine andere Technologie zuvor gleichermaßen in lokalen Netzen und in Weitverkehrsnetzen eingesetzt. Im WAN stellt sie die Basis der Breitbandkommunikation her, im LAN ermöglicht sie den Übergang zum WAN und gestaltet in Inhouse-Netzen ein leistungsfähigeres Transportsystem. Durch die durchgängige Einsetzbarkeit der ATM- Technik entfallen komplexe Protokollkonvertierungen beim Übergang vom LAN ins WAN und vielleicht irgendwann auch die Abgrenzung zwischen LAN und WAN. Die technisch bedingten Unterschiede zwischen den lokalen und Fernnetzebenen nehmen als Folge der vollständigen Digitalisierung des gesamten Netzes ab. $\mathrm{Zu}$ dem unterstützt ATM den Trend des Ausbaus der LANs zu Hochgeschwindigkeitsnetzen.

\section{A.2 BESTIMMUNG VON HAUPT- UND UNTERDETERMINANTE}

Berechnung der Hauptdeterminante anhand von (5.3.11):

$$
\begin{aligned}
|\Delta|= & \frac{\partial \alpha(1-\alpha)}{\partial p_{1}} x_{2}\left(p_{2}\right) * \frac{\partial \alpha(1-\alpha)}{\partial p_{2}} x_{1}\left(p_{1}\right) \\
& -\left[\begin{array}{l}
-\frac{\partial \alpha(1-\alpha)}{\partial p_{1}} x_{1}\left(p_{1}\right)+\alpha(1-\alpha) \frac{\partial x_{1}}{\partial p_{1}} \\
*\left(-\frac{\partial \alpha(1-\alpha)}{\partial p_{2}} x_{2}\left(p_{2}\right)+\alpha(1-\alpha) \frac{\partial x_{2}}{\partial p_{2}}\right)
\end{array}\right]
\end{aligned}
$$

Bestimmung der Unterdeterminanten:

$$
\begin{aligned}
\left|\Delta_{1}\right| & =\left|\begin{array}{rr}
-\frac{\partial A_{1}}{\partial p_{1}}+\frac{\partial D_{1}}{\partial p_{1}} c_{0} & -\frac{\partial D_{2}}{\partial p_{2}} \\
-\frac{\partial A_{2}}{\partial p_{2}}+\frac{\partial D_{2}}{\partial p_{2}} c_{0} & \frac{\partial G_{2}}{\partial p_{2}}
\end{array}\right| \\
& =\left(-\frac{\partial A_{1}}{\partial p_{1}}+\frac{\partial D_{1}}{\partial p_{1}} c_{0}\right) * \frac{\partial G_{2}}{\partial p_{2}}-\left[\left(-\frac{\partial A_{2}}{\partial p_{2}}+\frac{\partial D_{2}}{\partial p_{2}} c_{0}\right) *-\frac{\partial D_{2}}{\partial p_{2}}\right] \neq 0
\end{aligned}
$$




$$
\begin{aligned}
\left|\Delta_{2}\right| & =\left|\begin{array}{cc}
\frac{\partial G_{1}}{\partial p_{1}} & -\frac{\partial A_{1}}{\partial p_{1}}+\frac{\partial D_{1}}{\partial p_{1}} c_{0} \\
-\frac{\partial D_{1}}{\partial p_{1}} & -\frac{\partial A_{2}}{\partial p_{2}}+\frac{\partial D_{2}}{\partial p_{2}} c_{0}
\end{array}\right| \\
& =\frac{\partial G_{1}}{\partial p_{1}} *\left(-\frac{\partial A_{2}}{\partial p_{2}}+\frac{\partial D_{2}}{\partial p_{2}} c_{0}\right)-\left[-\frac{\partial D_{1}}{\partial p_{1}} *\left(-\frac{\partial A_{1}}{\partial p_{1}}+\frac{\partial D_{1}}{\partial p_{1}} c_{0}\right)\right] \neq 0
\end{aligned}
$$

Berechnung der Unterdeterminanten:

$$
\left.\begin{array}{rl}
\left|\Delta_{1}\right|= & -\left[\frac{\partial \alpha}{\partial p_{1}} x_{1}\left(p_{1}\right)\left[p_{1}-c_{0}-c_{1}\right]+\alpha \frac{\partial x_{1}}{\partial p_{1}}\left[p_{1}-c_{0}-c_{1}\right]\right] \\
& +\frac{\partial \alpha(1-\alpha)}{\partial p_{1}} x_{1}\left(p_{1}\right) c_{0}+\alpha(1-\alpha) \frac{\partial x_{1}}{\partial p_{1}} c_{0} * \frac{\partial \alpha(1-\alpha)}{\partial p_{2}} x_{1}\left(p_{1}\right) \\
& -\left[\begin{array}{l}
\left.-\frac{\partial(1-\alpha)}{\partial p_{2}} x_{2}\left(p_{2}\right)\left[p_{2}-c_{0}-c_{2}\right]+(1-\alpha) \frac{\partial x_{2}}{\partial p_{2}}\left[p_{2}-c_{0}-c_{2}\right]\right] \\
+\frac{\partial \alpha(1-\alpha)}{\partial p_{2}} x_{2}\left(p_{2}\right) c_{0}+\alpha(1-\alpha) \frac{\partial x_{2}}{\partial p_{2}} c_{0}
\end{array}\right] \\
& *-\left[\frac{\partial \alpha(1-\alpha)}{\partial p_{2}} x_{2}\left(p_{2}\right)+\alpha(1-\alpha) \frac{\partial x_{2}}{\partial p_{2}}\right] \\
\left|\Delta_{2}\right|= & \frac{\partial \alpha(1-\alpha)}{\partial p_{1}} x_{2}\left(p_{2}\right) *-\left[\frac{\partial \alpha}{\partial p_{1}} x_{1}\left(p_{1}\right)\left(p_{1}-c_{0}-c_{1}\right)+\alpha \frac{\partial x_{1}}{\partial p_{1}}\left(p_{1}-c_{0}-c_{1}\right)\right] \\
& +\left(\frac{\partial \alpha(1-\alpha)}{\partial p_{2}} x_{2}\left(p_{2}\right)+\alpha(1-\alpha) \frac{\partial x_{2}}{\partial p_{2}}\right) c_{0} \\
& +\left[\frac{\partial \alpha(1-\alpha)}{\partial p_{1}} x_{1}\left(p_{1}\right)+\alpha(1-\alpha) \frac{\partial x_{1}}{\partial p_{1}}\right] \\
& *\left[\frac{\partial \alpha}{\partial p_{1}} x_{1}\left(p_{1}\right)\left(p_{1}-c_{0}-c_{1}\right)+\alpha \frac{\partial x_{1}}{\partial p_{1}}\left(p_{1}-c_{0}-c_{1}\right)\right] \\
\left.-\left(\frac{\partial \alpha(1-\alpha)}{\partial p_{1}} x_{1}\left(p_{1}\right)+\alpha(1-\alpha) \frac{\partial x_{1}}{\partial p_{1}}\right) c_{0}\right]
\end{array}\right]
$$




\section{A.3 MONOPOLPREISE BEI SEHR HOHEM PRODUKT- DIFFERENZIERUNGSGRAD ${ }^{4}$}

Es gilt zu beweisen: $\Pi_{1}+\Pi_{2}=\Pi_{\text {gesamt }}=p^{M}$.

Für die Bestimmung von $p^{M}$ werden die Ergebnisse aus der individuellen nichtkooperativen Gewinnmaximierung aufaddiert unter den Annahmen, daß $\alpha=(1-\alpha)$ und $a_{1}=a_{2}=a$.

$$
\begin{aligned}
p^{M}= & \max _{p_{1}}\left\{x_{1}\left(p_{1}\right)\left[p_{1}-c_{0}-c_{1}\right]+\frac{1}{2}\left[x_{2}\left(p_{2}\right) a-x_{1}\left(p_{1}\right)\left(c_{0}+a\right)\right]\right\} \\
& +\max _{p_{2}}\left\{x_{2}\left(p_{2}\right)\left[p_{2}-c_{0}-c_{2}\right]+\frac{1}{2}\left[x_{1}\left(p_{1}\right) a-x_{2}\left(p_{2}\right)\left(c_{0}+a\right)\right]\right\}
\end{aligned}
$$

Angenommen, daß $x_{1}\left(p_{1}\right)=x_{2}\left(p_{2}\right)=x(p)$ und $c_{1}=c_{2}=c$, dann gilt:

$$
p^{M}=\operatorname{agg} \max _{p}\left\{x(p)\left[p-c_{0}-c\right]-\frac{1}{2} x(p) c_{0}\right\} \text {. }
$$

Für die Bestimmung von $\Pi_{1}+\Pi_{2}=\Pi_{\text {gesam }}$ gelten obige Annahmen auch hier:

$$
\Pi_{\text {gesamt }}=\left\{x(p)\left[p-c_{0}-c\right]-\frac{1}{2} x(p) c_{0}\right\} \text {. }
$$

Daraus folgt:

$$
\max _{p} \Pi_{\text {gesamt }}=p_{\text {ges }}^{*}=p^{M} .
$$

D.h. der Preis, der sich unter kooperativer gemeinsamer Gewinnmaximierung ergibt, entspricht dem Preis, der sich ergeben würde, wenn jeder Anbieter wie ein Monopolist agiert und nicht kooperativ seinen Gewinn maximiert. Bei einheitlichen Zusammenschaltungsentgelten wird ein dereguliertes Monopol Zusammenschaltungsentgelte wählen, die den Monopolpreisen entsprechen und Unternehmen befähigen, gemeinsame Gewinne zu maximieren. ${ }^{5}$ Das Ergebnis ändert sich nicht, wenn die Zahl an Netzbetreibern zunimmt.

${ }^{4}$ Vgl. Carter/Wright (1996), S. 20.

${ }^{5}$ Vgl. Carter/Wright (1999), S. 8. 
Sarah Eliza Schniewindt - 978-3-631-75130-5 Downloaded from PubFactory at 01/11/2019 07:57:00AM via free access 


\section{LITERATURVERZEICHNIS}

Abbott, L. (1958): Qualităt und Wettbewerb: Ein Beitrag zur Wirtschaftstheorie, München u. Berlin.

Albach, H; Knieps, G. (1997): Kosten und Preise in wettbewerblichen Ortsnetzen, Freiburger Studien zur Netzökonomie, Bd. 2, Baden-Baden.

Akerlof, G.A. (1970): The Market for ,Lemons': Quality Uncertainty and the Market Mechanism, in: Quarterly Journal of Economics, Bd. 84, S. 488-500.

Armstrong, M. (1996): Network Interconnection, Discussion Paper in Economics and Econometrics Heft 9625, Department of Econonmics, University of Southampton, United Kingdom.

Armstrong, M. (1997): Competition in Telecommunications, in: Oxford Review of Economic Policy, Bd. 13, S. 64-82.

Armstrong, M. (1998): Network Interconnection in Telecommunications, in: Economic Journal, Bd. 108, S. 545-654.

Armstrong, M.; Doyle. C. (1995): The Economics of Access Pricing, Vortrag gehalten auf dem Workshop No. 2 on Economics on Information Society; 1415 December, Ystanbul. OECD Report:

http://www.oecd.org/daf/clp/bdpt205.htm.

Armstrong, M.; Doyle. C.; Vickers, J. (1996): The Access Pricing Problem: A Synthesis, in: The Journal of Industrial Economics, Bd. 44, Heft 2, S. 131-150.

Armstrong, M.; Vickers, J. (1998): The Access Pricing Problem with Deregulation: A Note, in: The Journal of Industrial Economics, Bd. 46, Heft 1; S. 115121.

Arnbak, J.; Mitchell; B.; Neu, W.; Neumann, K.H.; Vogelsang, I. (1994): Network Interconnection in the Domain of ONP, Study for DGXIII of the European Commission, Final Report; WIK/EAC, Bad Honnef.

Arndt, H. (1958): Anpassung und Gleichgewicht am Markt, in: Jahrbuch für Nationalökonomie und Statistik, Bd. 170, S. 217-228. 
Arrow, K.J. (1991): The Economics of Agency, in: J.W.Pratt, R.J. Zeckhauser (Hrsg.), Principals and Agents. The Structure of Business, erw. Aufl., Boston, S. 37-51.

Arthur, W.B. (1988a): Self- Reinforcing Mechanisms in Economics, in: P.W. Anderson, K. J. Arrow, D. Pines (Hrsg.), The Economy as an Evolving Complex System: The Proceedings of the Evolutionary Paths of the Global Economy Workshop, gehalten im Sept. 1987 in Santa Fe, New Mexiko, Redwood City, Calif.

Arthur, W.B. (1988b): Competing Technologies: An Overview, in: G. Dosi u.a. (Hrsg.), Technical Change and Economic Theory, London.

Arthur, W.B. (1989): Competing Technologies, Increasing Returns and Lock-In by Historical Events, in: The Economic Journal, Bd. 99, S. 116-131.

Baake, P. (1996): Netzexternalitäten und strategisches Verhalten, Aachen.

Bain, J.S. (1956): Barriers to New Competition, Cambridge, Mass.

Bain, J.S. (1968): Industrial Organization, New York.

Baker, S.; Wu, L. (1998): Applying the Market Definition Guidelines of the European Commission, NERA Topics, Heft 21.

Baron, D.; Myerson, R. (1981): Regulating a Monopolist with Unknown Costs, in: Econometrica, Bd. 50, S. 911-930.

Baumol, W.J. (1983): Some Subtle Issues in Railroad Deregulation, in: International Journal of Transport Economics, Bd. 10, S. 431-355.

Baumol, W.J. (1999): Interconnection Prices: Making Telephone Competition a Reality, in: Japan and the World Economy, Bd. 11; S. 575-577.

Baumol, W.J.; Bradford, D.F. (1970): Optimal Departures from Marginal Cost Pricing, in: The American Review, Bd. 60, S. 265-283.

Baumol, W.J.; Ordover, J.A. (1992): Antitrust: Source of Dynamic and Static Inefficiencies?, in: T.M. Jorde und D.J. Teece (Hrsg.), Antitrust, Innovation and Competitiveness, N.Y., Oxford, S. 82-97. 
Baumol, W.J.; Panzar, J.C.; Willig, R.D. (1988): Contestable Markets and the Theory of Industry Structure, erw. Aufl., Orlando.

Baumol, W.J.; Sidak; J.G. (1994a): The Pricing of Inputs Sold to the Competitors, in: Yale Journal on Regulation, Bd. 11, Heft 1, S. 171-202.

Baumol, W.J.; Sidak; J.G. (1994b): Toward Competition in Local Telephony, Cambridge, Mass.

Baumol, W.J.; Sidak. J.G. (1995): Stranded Costs, in: Harvard Journal of Law and Public Policy, Bd. 18, S. 835-849.

BellSouth/INDETEC International/Sprint/US West (Hrsg.) (1998): Benchmarkt Cost Proxy Model Release 3.1: Model Methodology.

Berg, S.V.; Tschirhart, J. (1988): Natural Monopoly Regulation, Cambridge, Mass.

Berthes, G.; Hochmuth, C.; Lohscheller, H. (1998): Ausbau von Kabel-TVNetzen, in: Telekom Praxis, Heft 10, S. 24-35.

Besen, S.M. ; Farrell, J. (1994): Choosing How to Compete: Strategies and Tactics in Standardization, in: Journal of Economic Perspectives, Bd. 8, Heft 2, S. 117-31.

Blankart, C.B.; Knieps, G. (1991): Path Dependence, Network Externalities and Standardization, Diskussionspapier Nr. 151 der Technischen Universität Berlin.

Blankart, C.B.; Knieps, G. (1992): Netzökonomik, in: Jahrbuch für neue politische Ökonomie, Nr.11, S. 73-87.

Blankart, C.B.; Knieps, G. (1995 ): Market-oriented Open Network Provision, in: Information Economics and Policy, Bd. 15, S. 183-296.

Blankart, C.B.; Knieps, G. (1996): Regulierung von Netzen, in: ifo-Studien 1996, Bd. 42, Heft 4, München, S. 483-504.

Bös, D. (1981): Economic Theory of Public Enterprise, Berlin u.a. 
Boiteux, M. (1971): On the Management of Public Monopolies Subject to Budgetary Constraints, in: Journal of Economic Theory, Bd. 3, S. 219-240.

Bolton, P.; Brodley, J.F.; Riordan, M. H. (2000): Predatory Pricing: Strategic Theory and Legal Policy, in Georgetown Law Journal, Bd. 88, S. 2239-2330.

Bolton, P.; Scharfstein D.S. (1990): A Theory of Predation based on Agency Problems in Financial Contracting, in: American Economic Review, Bd. 80, S. 93-106.

Borchers, E. (1999): Keine Vision, sondern Wirklichkeit: Power Line Communication mit $2 \mathrm{Mbit} / \mathrm{s}$, in: Telekom Praxis, Heft 5, S. 34-42.

Borrmann, J.; Finsinger, J. (1999): Markt und Regulierung, München.

Braeutigam, R.R. (1980): An Analysis of Fully Distributed Cost Pricing in Regulating Industries, in: The Bell Journal of Economics, Bd. 11, S. 182-196.

Braeutigam, R.R. (1998): Optimal Policies for Natural Monopolies, in: R. Schmalensee, R.D. Willig (Hrsg.), Handbook of Industrial Organization, 5. Aufl., Amsterdam.

Brennan, T. J. (1996): Making Economic Sense of the Telecommunications Act of 1996, in: Industrial and Corporate Change, Bd. 5, Heft 4, S. 941-63.

Breyer, F.; Zweifel, P. (1997): Gesundheitsökonomie, 2. Aufl., Heidelberg.

Büchner, W. (Hrsg.) (2000): Beck'scher TKG-Kommentar, 2. Aufl., München.

Bühler, S. (2000): A Further Look at Two-Way Network Competition in Telecommunications, Vortrag gehalten auf der $26^{\text {th }}$ Annual Conference of the European Association for Research in Industrial Economics (EARIE) 1999 in Turin.

Burr, W. (1995): Wettbewerb in der Telekommunikation, Wiesbaden.

Cabral, L.; Salant, D.; Woroch, G. (1994): Monopoly Pricing with Network Externalities, in: ewp - io / 9411003. 
Carter, M.; Wright, J. (1996): Interconnection in Network Industries, Discussion Paper Heft 9607 of the Department of Economics, University of Canterbury, Christchruch, New Zealand.

Carter, M.; Wright, J. (1999): Bargaining over Interconnection: The ClearTelecom Dispute, in: The Economic Record, Bd. 75; Heft 230, S. 241-55.

Cave, M. (1994): Interconnection Issues in UK Telecommunications, in: Utilities Policy, Bd. 4, Heft 3, S. 215-222.

Cave, M. (1995): Alternative Telecommunications Infrastructures: Their Competition Policy and Market Structure Implications, OECD Report, in: http://www.oecd.org/daf/clp/bdpt207.htm

Cave, M. (1997): The Evolution of Telecommunications Regulation in the UK, in: European Economic Review, Bd. 41, S. 691-699.

Cave, M., Crowther, P.; Hancher, L. (1995): Competition Aspects of Access Pricing. Report to the European Commision, Brussel.

Cave, M.; Doyle, C. (1994): Access Pricing in Network Utilities in Theory and Practice, in: Utilities Policy, Bd. 4, S. 181-190.

Cave, M.; Lever, K.; Mills, R.; Trotter, S. (1990): Cost Allocation and Regulatory Pricing in Telecommunications, in: Telecommunications Policy, Bd. 14, S. 505-520.

Cave, M.; Prosperetti, L. (2000): The Liberalisation of European Telecommunications: A Critical Review and Future Progress, Vortrag gehalten auf der $11^{\text {th }}$ European Regional Conference of the International Telecommunications Society (ITS), 10-12 September, 2000, Lauzanne.

Cave, M.; Prosperetti, L. (2001): European Telecommunications Infrastructures, in: Oxford Review of Economic Policy, Bd. 17, Heft 3, S. 416-431.

Chamberlin, E.H. (1933): The Theory of Monopolistic Competition, Cambridge.

Chiang, A.C. (1984): Fundamental Methods of Mathematical Economics, New York. 
Chou, C.; Shy, O. (1996): Do Consumers Gain or Lose when More People buy the Same Brand?, in: M.J. Holler und J.-F. Thisse (Hrsg.), The Econonmics of Standardization, Special Issue des European Journal of Political Economy, Bd. 12, S. 309-330.

Coase, R. (1960): The Problem of Social Cost, in: Journal of Law and Economics, Bd. 3, S. 1-44.

Cramer, C. (1994): Local Competition for Telephone Services, in: Review of Industrial Organization, Bd. 9, S. 273-291.

Crew, M.A.; Kleindorfer, P.R. (1999): Stranded Assets in Network Industries in Transition, in: M.A. Crew (Hrsg.), Regulating under Increasing Competition, Boston u.a., S. 63-78.

Crew, M.A.; Kleindorfer, P.R. (2002): Regulatory Economics: Twenty Years of Progress? in: Journal of Regulatory Economics, Bd. 21, Heft 1, S. 5-22.

David, P.A. (1985): Clio and the Economics of QWERTY, in: American Economic Review, Papers and Proceedings, Bd. 75, Heft 2, S. 332-337.

David, P.A. (1987): Some new Standards for the Economics of Standardization in the Information Age, in: P. Dagputa, P. Stoneman (Hrsg.), Economic Policy and Technological Performance, Cambridge.

David, P.A.; Bunn, J.A. (1988): The Economics of Gateway Technologies and Network Evolution: Lessons from Electricity Supply History, in: Information Economics and Policy, Bd. 3, Heft 2, S. 165-202.

Demsetz, H. (1968): Why regulate Utilities, in: Journal of Law and Economics, Bd. 11, S. 55-67.

Dippon, C.M. (2001): Competitive Pricing Methodologies for Wholesale Broadband Services, Vortrag gehalten auf der $12^{\text {th }}$ European Regional Conference of the International Telecommunications Society (ITS), 1-3 September 2001 in Dublin.

Distelkamp, M. (1999): Möglichkeiten des Wettbewerbs im Orts- und Anschlußbereich des Telekommunikatiosnetzes, WIK Diskussionsbeitrag Nr. 196, Bad Honnef. 
Doganoglu, T.; Taumann, Y. (1998): Network Competition and Acess Charge Rules, Vortrag gehalten auf der $28^{\text {th }}$ Annual Conference of the European Association for Research in Industrial Economics (EARIE), 27 August- 1 September 2001 in Dublin.

Doll, R.; Wieck, R. (1998a): Analytische Kostenmodelle als Grundlage für Entgeltregulierungsentscheidungen, in: MultiMedia und Recht, Bd. 1, Heft 6, S. 280-287.

Doll, R. ; Wieck, R. (1998b): Eingeschränkte Aussagekraft von analytischen Kostenmodellen - Eine Replik, in: MultiMedia und Recht, Bd. 1, Heft 12, S. 659662.

Donges, J.B.; Freytag, A. (2001): Allgemeine Wirtschaftspolitik, Stuttgart.

Dunleavy, P.; Hood, C. (1994): From Old Public Administration to New Public Management, in: Public Money \& Management, Heft July/September, S. 9-16.

Economides, N. (1989): Desirability of Compatibility in the Absence of Network Externalities, in: The American Economic Review, Bd. 79, Heft 5, S. 11651181.

Economides, N. (1994): A Monopolist's Incentive to Invite Competitors to Enter in Telecommunication Services, in: G. Pogorel(Hrsg.), Global Telecommunications Strategies and Technological Changes, Amsterdam.

Economides, N. (1996a): The Economics of Networks, in: International Journal of Industrial Organization, Bd. 14, Heft 2, S. 673-699.

Economides, N. (1996b): Network Externalities, Complementarities, and Invitations to Enter, in: European Journal of Political Economy, Bd. 12, 1996, S. 211-33.

Economides, N. (1998a): The Incentive for Non- Price Discrimination by an Input Monopolist, in: International Journal of Industrial Organization, Bd. 16, S. 271284.

Economides, N. (1998b): The Telecommunications Act of 1996 and its Impact, Working Paper Heft 99-003, N.Y. University, Center for Law and Business, New York. 
Economides, N. (2000): Real Options and the Costs of the Local Telecommunications Network, in: J. Alleman, E. Noam (Hrsg.): The New Investment Theory of Real Options and its Implications for the Cost Models in Telecommunications, Boston u.a.

Economides, N.; Flyer, F. (1997): Compatibility and Market Structure for Network Goods. Discussion Paper of the Stern School of Business, New York University, New York.

Economides, N.; Himmelberg, C. (1994): Critical Mass and Network Evolution in Telecommunications, in: G.W. Brock (Hrsg.), Toward a Competitive Telecommunication Industry. Selected Papers from the 1994 Telecommunications Policy Research Conference, New Jersey.

Economides, N.; Salop, S.C. (1992): Competition and Integration Among Complements and Network Market Structure, in: The Journal of Industrial Economics, Bd. 40, Heft 1, S. 105-23.

Economides, N.; White, L.J. (1994): Networks and Compatibility: Implications for Antitrust, in: European Economic Review, Bd. 38, S. 651-662.

Economides, N.; White, L.J. (1995): Access and Interconnection Pricing: How Efficient is the "ECPR", in: The Antitrust Bulletin, Bd. 40, S. 557-579.

Economides, N.; White, L.J. (1996): Access and Interconnection Pricing: How Efficient is the "ECPR", in: G.W.Brock, G. L. Rosston (Hrsg.), The Internet and Telecommunications Policy. Selected Papers from the 1995 Telecommunications Policy Research Conference, New Jersey, S. 45-61.

Economides, N.; White, L.J. (1998): The Inefficiency of the ECPR yet again: A Reply to Larson, in: Antitrust Bulletin, Bd. 43, Heft 2, S. 429-444.

Economides, N.; Lopomo, G.; Woroch, G. (1996): Regulatory Pricing Rules to Neutralize Network Dominance, in: Industrial and Corporate Change, Bd. 5, Heft 4, S. 1013-1028.

Ewers, M. (1997): Netzwettbewerb und Netzzugang: die Sicht eines neuen Wettbewerbers, in: Wettbewerbspolitik in deregulierten Verkehrsmärkten. Schriftenreihe der Deutschen Verkehrswissenschaftlichen Gesellschaft e.V., Hinterzarten, S. 105-116. 
Farrell, J. (1987): Information and the Coase Theorem, in: Economic Perspectives, Bd.1, Heft 2, S. 113-129.

Farrell, J.; Gallini, N. (1988): Second-Sourcing as a Commitment: Monopoly Incentives to Attract Competition, in: Quarterly Joural of Economics, Bd. 103, S. 673-694.

Farrell, J.; Saloner, G. (1985): Standardization, Compatibility and Innovation, in: Rand Journal of Economics, Bd. 16, Heft1, S.70-83.

Farrell, J.; Saloner, G. (1986a): Installed Base and Compatibility: Innovation, Product Preannouncement and Predation, in: American Economic Review, Bd. 76, Heft 5, S. 940-955.

Farrell, J.; Saloner, G. (1986b): Standardization and Variety, in: Economic Letters, Bd. 20, Heft 1, S. 71-74.

Farrell, J.; Saloner, G. (1992): Compatibility and the Control of Interfaces, in: The Journal of Industrial Economics, Bd. 40, Heft 1, S. 9-33.

FAZ : Pressemitteilung vom 11.05.2000 zu City Carrier.

Federal Commission of Communications (FCC) (1996): First Report and Order - In the Matter of: Implementation of the Local Competition Provisions in the Telecommunications Act of 1996 and Interconnection between Local Exchange Carriers and Commercial Mobile Radio Service Providers, Washington.

Federal Commission of Communications (FCC) (1999): Trends in Telephone Service, Washington.

Fehl, U. (1985): Das Konzept der Contestable Markets und der Marktprozeß, in: G. Bombach u.a. (Hrsg.), Industrieökononik: Theorie und Empirie, Tübingen, S. 29-49.

Feichtinger, T. (1998): Topologien von City- und Regionalnetzen mit besonderer Berulcksichtigung des sekundären Anschlußnetzes, in: Telekom Praxis, Heft 12, S. 20-24.

Finsinger, J. (1991) Wettbewerb und Regulierung, München. 
Fritsch, M.; Wein, T.; Ewers, H.-J. (2001): Marktversagen und Wirtschaftspolitik: Mikroökonomische Grundlagen staatlichen Handelns, 4. Aufl., München.

Fundneider, O. (1994): Synchrone Digitalhierarchie und Asynchroner Transfermodus, in: Telekom Praxis, Heft 4, S. 29-36.

Gabel, D.J. (1991): An Application of Stand-Alone Costs to the Telecommunications Industry, in: Telecommunications Policy, Bd.15, Heft 1, S. 75-84.

Gabel, D.; Kennet, M.D. (1991): Estimating the Cost Structure of the Local Telephone Exchange Network, Study prepared for the National Regulation Research Institute (NRRI), Columbus, Ohio, October 1991.

Gabel, D.; Kennet, M.D. (1994): Economies of Scope in the Local Telephone Exchange Market, in: Journal of Regulatory Economics, Bd. 6, S. 381-398.

Gabel, D.; Kennet, M.D. (1997): The Effect of Cellular Service on the Cost Structure of a Land-Based Telephone Network, in: Telecommunications Policy, Bd. 21, S. 411-422.

Gabelmann, A. (2001): Regulating European Telecommunications Markets: Unbundled Access to the Local Loop outside Urban Areas, in: Telecommunication Policy, Bd. 25, S. 729-741.

Gans, J.S.; King, S.P. (1999): Using ,Bill and Keep“ Interconnect Arrangements to Soften Network Competition. Melbourne Business School Working Paper Heft 99-12.

Gans, J. S.; King, S.P. (2000): Regulating Termination Charges for Telecommunications Networks, Discussion Paper of the Melbourne Business School and the Department of Economics, in: www.mbs.unimelb.edu.au/jgans/reearch.htm.

Garcia-Martin, J.A. (2001): Stranded Costs: An Overview, Working Paper Heft 0108, University Pompeu Fabra and CEMFEI.

Gasmi, F.; Laffont, J.J.; Sharkey, W.W. (1999): Empirical Evaluation of Regulatory Regimes in Local Telecommunications Markets, in: Journal of Economics an Management Strategy, Bd. 8, Heft 1, S. 61-94. 
Gerpott, T.J. (1998): Wettbewerbsstrategien im Telekommunikationsmarkt, 3. Aufl., Stuttgart.

Gerpott, T.J. (1999): Strukturwandel des deutschen Telekommunikationsmarktes, in: Handbuch Telekommunikation und Wirtschaft, München.

Gerpott, T.J.; Walter, A.; Gemmel, D. (1998): Preisvergleiche für StandardFestverbindungen, in: Zeitschrift für Betriebswirtschaft, Nr. 68, S. 1273-1302.

Gilbert, R.J. (1992): Symposium on Compatibility: Incentives and Market Structure, in: The Journal of Industrial Economics, Bd. 40, Heft 1 , 1992, S. 18.

Goldbeck, U; Ortiges, M. (1998): Die letzte Meile überwinden. Migrationskonzept fur Citynetze, in: Telekom Praxis, Heft 6, S. 38-40.

Graack, C. (1997): Telekommunikationswirtschaft in der EU, Heidelberg.

Graubner, J. (1999): Wilreless Local Loop: Eine Alternative zum Draht, in: Telekom Praxis, Heft 3, S. 38-43.

Gravelle, H.; Rees, R. (1992): Microeconomics, 2. Aufl., Harlow and New York.

Gröhn, A. (1997): Ein Modell der Netzwerkeffekte in der Software-Industrie. Kieler Arbeitspapiere Nr. 790 des Instituts für Weltwirtschaft an der Universităt Kiel.

Gröhn, A. (1999): Netzwerkeffekte und Wettbewerbspolitik. Eine ökonomische Analyse des Softwaremarktes, Tübingen.

Gröner, H.; Kőhler, H.; Knorr, A. (1995): Liberalisierung der Telekommunikationsmärkte. Wettbewerbspolitische Probleme des Markteintritts von Elektrizitätsversorgungsunternehmen in die deutschen Telekommunikationsmärkte, Bern, Stuttgart, Wien.

Haaß, W.D. (1997): Handbuch der Kommunikationsnetze, Berlin.

HAI (1998): HAI Model Release 5.0a, Model Description, Boulder.

Herdzina, K. (1999): Wettbewerbspolitik, 5. Aufl., Stuttgart. 
Höckels, A. (2001a): Alternative Formen des entbündelten Zugangs zur Teilnehmeranschlußleitung. Diskussionsbeitrag Nr. 215 des Wissenschaftlichen Institutes für Kommunikationsdienste, Bad Honnef.

Höckels, A. (2001b): Internationaler Vergleich der Wettbewerbsentwicklung im Local Loop, Diskussionsbeitrag Nr. 228 des Wissenschaftlichen Institutes für Kommunikationsdienste, Bad Honnef.

Hoffmann-Riem, W.; Schulz, W.; Held, T (2000): Konvergenz und Regulierung: Optionen für rechtliche Regelungen und Aufsichtsstrukturen im Bereich Information, Kommunikation und Medien, Baden-Baden.

Holler, M. (1997): Die Modellierung von Netzeffekten und Ansätze industriepolitischer Steuerung, in: Jahrbuch für Neue Politische Ökonomie, Bd. 16, S. 90114.

Holmström, B.; Milgrom, P. (1991): Multitask Principal- Agent Analysis: Incentive Contracts, Asset Ownership, and Job Design, in: Journal of Law, Economics and Organizations, Bd. 7, S. 24-52.

Hood, C. (1995): The „New Public Managmenet" in the 1980's: Variations on a Theme, in: Accounting, Organizations and Society, Bd. 20, S. 93-109.

Horn, M.; Knieps, G.; Müller, J. (1988): Deregulierungsmaßnahmen in den USA: Schlußfolgerungen für die BRD. Gutachten des DIW, Baden-Baden.

Hotelling, H. (1929): Stability in Competition, in: Economic Journal, Bd. 39, S. 41-57.

Huber, B. (1999): ADSL - Technik und Anwendungen bei der Deutschen Telekom, in: Telekom Praxis, Heft 4, S. 30-36.

Ickenroth, B.; Schmidt, F. (1997): Kostenmodelle für Telekommunikationsortsnetze, Newsletter Nr. 29 des Wissenschaftliches Institut für Kommunikationsdienste (WIK) im Dezember 1997, Bad Honnef.

ITK: http://www.itk.de/voip/index.html

Jung, V.; Warnecke, H.-J. (1998): Handbuch für Telekommunikation, Berlin, Heidelberg u.a. 
Kabel-Berlin: http://www.kabel-berlin.de

Kahn, A.E. (1989): The Econonmics of Regulation. Principles and Institutions. Vol 1, 2. Aufl., Cambridge u.a.

KallfaB, H.H. (1997): Konzepte und Indikatoren zur Abgrenzung räumlicher Mărkte in der europäischen Zusammenschlußkontrolle, in: Wettbewerbspolitik im Spannungsfeld nationaler und internationaler Kartellrechtsordnungen. Festschrift für Ingo Schmidt zum 65. Geburtstag, Baden-Baden, S.111-129.

Kantzenbach, E. (1967): Die Funktionsfähigkeit des Wettbewerbs, 2. Aufl., Göttingen.

Kantzenbach, E.; Krüger, R. (1990): Zur Frage der richtigen Abgrenzung des sachlich relevanten Marktes bei der wettbwerbspolitischen Beurteilung von Unternemenszusammenschlussen, in: Wirtschaft und Wettbewerb, Jg. 40, S. 472-481.

Katz, M. L.; Shapiro, C. (1985): Network Externalities, Competition and Compatibility, in: American Economic Review, Bd. 75, Heft 3; S. 424-440.

Katz, M. L.; Shapiro, C. (1986a): Product Compatibility Choice in a Market with Technological Progress, in: Oxford Economic Papers, Bd. 38 (Suppl.), S. 146-165.

Katz, M. L.; Shapiro, C. (1986b): Technology Adoption in the Presence of Network Externalities, in: Journal of Political Economy, Bd. 94, Heft 4, S. 822841.

Katz, M. L.; Shapiro, C. (1994): Systems Competition and Network Effects, in: Journal of Economic Perspectives, Bd. 8, Heft 2, S. 93-115.

Kaysen, C.; Turner, D.F. (1959): Antitrust Policy: An Econonmic and Legal Analysis, Cambridge.

Kiener, S. (1990): Die Principal-Agent- Theorie aus informationsökonomischer Sicht, Heidelberg.

Klaue, S.; Schwintowski, H.-P. (2001): Marktabgrenzung und Marktbeherrschung im Telekommunikationssektor, Baden-Baden. 
Kleinemeyer, J. (1997): Standardisierung zwischen Kooperation und Wettbewerb - Eine spieltheoretische Betrachtung, Hamburg.

Klodt, H.; Laaser, C.-F.; Lorz, J.O.; Maurer, R. (1995): Wettbewerb und Regulierung in der Telekommunikation, Tübingen.

Knieps, G. (1998a): Ein analytisches Kostenmodell für das Ortsnetz - Referenzmodell: Stellungnahme und Kommentare, Diskussionspapier Nr. 48 des Instituts für Verkehrswissenschaft und Regionalpolitik an der Universität Freiburg.

Knieps, G. (1998b): Der Irrweg analytischer Kostenmodelle als regulatorische Schattenrechnung, in: MultiMedia und Recht, Bd. 1, Heft 11, S. 598-602.

Knieps, G. (1999): Interconnection and Network Access: The Case of Telecommunications, Diskussionspapier Nr. 56 des Instituts für Verkehrswissenschaft und Regionalpolitik an der Universität Freiburg.

Knieps, G.; Sommer, H. (1988): Kostenaufteilung bei Mehrzweckprojekten, in: Schweizerische Zeitung für Volkswirtschaft und Statistik, Heft 2, S. 151-173.

Kolbe, L.A.; Tye, W.B. (1996): Compensation for the Risk of Stranded Costs, in: Energy Policy, Bd. 24, Heft 12, S. 1026-1050.

Köster, D. (1999): Wettbewerb in Netzproduktmärkten, Wiesbaden.

Kommission der Europäischen Gemeinschaften (1997): Bekanntmachung der Kommission über die Definition des relevanten Marktes im Sinne des Wettbewerbsrechts der Gemeinschaft, Amtsblatt der Europäischen Gemeinschaften Serie C 372, 12.09.1997.

Kommission der Europåischen Gemeinschaften (2000a): Entwurf einer Mitteilung der Kommission, Entbündelter Zugang zum Teilnehmeranschluß: wettbewerbsorientierte Bereitstellung einer vollständigen Palette von elektronischen Kommunikationsdiensten einschließlich mulimedialer Breitband- und schneller Internet- Dienste, Komm (2000) 237, Brüssel.

Kommission der Europäischen Gemeinschaften (2000b):Vorschlag für eine Richtlinie des Europäischen Parlaments und des Rates über den Zugang zu elektronischen Kommunikationsnetzen und zugehörigen Einrichtungen sowie deren Zusammenschaltung, Komm (2000) 384, Brüssel. 
Kommission der Europäischen Gemeinschaften (2000c): Sechster Bericht über die Umsetzung des Reformpaketes fur den Telekommunikationssektor. Mitteilung der Kommission an den Rat, das europäische Parlament, den Wirtschafts- und Sozialausschuß und den Ausschuß der Regionen. Komm (2000) 814 , Brüssel.

Kommission der Europäischen Gemeinschaften (2000d): Interconnection Tariffs in Member States as of 1st March 2000, verfügbar unter: www.ispo.cec.be

Kuhlenkampff, G. (2000): Der Markt für Internet-Telefonie - Rahmenbedingungen, Unternehmensstrategien und Marktentwicklung, WIK Diskussionsbeitrag Nr. 206, Bad Honnef.

Kruse, J. (1985): Ökonomie der Monopolregulierung, Göttingen.

Kruse, J. (1996): Liberalisierung der Telekommunikation in Deutschland, in: Wirtschaftsdienst, 1996 / II, S.73-80.

Kruse, J. (1997): Vertikale Integration als Wettbewerbsproblem, in: J. Kruse; K. Stockmann; L. Vollmer (Hrsg.), Wettbewerbspolitik im Spannungsfeld nationaler und internationaler Kartellrechtsordnungen, Baden-Baden, S. 247-270.

Kruse, J. (2000): Deregulierungsbedarf bei Ferngesprächen, in: Wirtschaftsdienst, Bd. 80., S. 402-209.

Kruse, J.; Haucap, J. (2002): Zuviel Wettbewerb in der Telekommunikation? Anmerkungen zum zweiten Sondergutachten der Monopolkommission, in: Wirtschaftsdienst, Bd. 82, Heft 2, S. 92-98.

Laffont, J.-J.; Martimort, D. (2002): The Theory of Incentives. The PrincipalAgent Model, Princeton and Oxford.

Laffont, J.-J.; Rey, P.; Tirole, J. (1997): Competition between Telecommunications Operators, in: European Economic Review, Bd. 41, Heft 3, S. 701-711.

Laffont, J.-J.; Rey, P.; Tirole, J. (1998): Network Competition I.: Overview and Nondiscriminatory Pricing, in: Rand Journal of Economics, Bd. 29, S.1-37.

Laffont, J.-J.; Tirole, J. (1986): Using Cost Observation to Regulate Firms , in: The Journal of Political Economy, Bd. 94, Heft 3, S. 614-641. 
Laffont, J.-J.; Tirole, J. (1994a): A Theory of Incentives in Procurement and Regulation, Cambridge u.a.

Laffont, J.-J.; Tirole, J. (1994b): Access Pricing and Competition, in: European Economic Review, Bd. 38, S. 1673-1710.

Laffont, J.-J.; Tirole, J. (1996): Creating Competition through Interconnection, in: Journal of Regulatory Economics, Bd. 10, S. 27-256.

Laffont, J.-J.; Tirole, J. (2000): Competition in Telecommunications, Cambridge.

Levin, D.; Mathews, J. (1998): Access Networks and Regulatory Measures. An Interim Report prepared by Ovum for DG XIII of the Commission of the European Union, London.

Liebowitz, S.J.; Margolis, S.E. (1990): The Fable of Keys, in: Journal of Law and Economics, Bd. 33, S. 1-25.

Liebowitz, S.J.; Margolis, S.E. (1994): Network Externality: An Uncommon Tragedy, in: Journal of Economic Perspectives, Bd. 8, Heft 2, S. 133-150.

Liebowitz, S.J.; Margolis, S.E. (1995): Are Network Externalities a New Source of Market Failure?, in: Reserach in Law and Economics, Bd. 17, S. 1-22.

Mahler, A.; Stoertzer, M.-W. (Hrsg.) (1995): Die Diffusion von Innovationen in der Telekommunikation, Berlin.

Matutes, C.; Padilla, A. J. (1994): Shared ATM Networks and Banking Competition, in: European Economic Review, Bd. 38, S. 1113-1138.

Matutes, C.; Regibeau, P. (1988): „Mix and Match“; Product Compatibility without Network Externalities, in : Rand Journal of Economics, Bd. 19, S. 221234.

Matutes, C.; Regibeau, P. (1992): Compatibility and Bundling of Complementary Goods in a Duopoly, in: The Journal of Industrial Economics, Bd. 40, Heft 1, S. 37-54. 
Matutes, C.; Regibeau, P. (1996): A Selective Review of the Economics of Standardization: Entry Deterrence, Technological Progress and International Competition, European Journal of Political Economy, Bd. 12, S. 183-209.

McKenzie, D.J.; Small, J.P. (1997): Econometric Cost Structures Estimates for Cellular Telephony in the United States, in: Journal of Regulators Economics, Bd. 12, Heft 2, S. 147-157.

Mellewigt, T.; Theissen, B. (1998): Bottom-up-Kostenmodelle als Kerninstrument furr zukünftige Entgeltregulierungsentscheidungen: eine Replik, in: MultiMedia und Recht, Bd. 1, Heft 11, S. 589-594.

Merkt, J. (1997): A Note on Unbundling Requirements for Telecommunications Networks, Diskussionsbeitrag Nr. 40 des Instituts furr Verkehrswissenschaften und Regionalpolitik der Albert-Ludwigs-Universităt Freiburg/Breisgau.

Merkt, J. (1998): Wettbewerb im Local Loop, Baden-Baden.

Ministry of Commerce und the Treasury of New Zealand (1995): Regulation of Access to Vertically-Integrated Natural Monopolies, Discussion Paper, Wellington, New Zealand, August 1995.

Mitchell, B.M. (1990): Interconnection of Telephone Access and Local Use, in: The Rand Corporation, R-3909-ICTF; Santa Monica.

Mitchell, B.M.; Vogelsang, I. (1991): Telecommunications Pricing: Theory and Practice, Cambridge.

Mitchell, F.; Neu,W.; Neumann, K.-H.; Vogelsang, I. (1994): The Regulation of Pricing Interconnection Services, in: G.W. Brock (Hrsg.), Toward a Competitive Telecommunication Industry. Selected Papers from the 1994 Telecommunications Policy Research Conference.

Möschel, W. (2001): Kommentar zu $\$ 19$ GWB, Marktbeherrschung, wettbewerbsbeschränkendes Verhalten, in: U. Immenga, E.-J. Mestmäcker (Hrsg.), Gesetz gegen Wettbewerbsbeschränkungen - GWB, Kommentar zum Kartellgesetz, 3. Aufl., München, S. 561-748.

Monopolkommission (1991): 20. Sondergutachten, Zur Neuordnung der Telekommunikation, Bonn. 
Monopolkommission (1996): 24. Sondergutachten, Die Telekommunikation im Wettbewerb, Bonn.

Monopolkommission (1998/99): 13. Hauptgutachten, Wettbewerbspolitik in Netzstrukturen, Bonn.

Monopolkommission (1999): 29. Sondergutachten, Wettbewerb auf Telekommunikations- und Postmärkten, Bonn.

Monopolkommission (2001): 33. Sondergutachten, Wettbewerbsentwicklung bei Telekommunikation und Post 2001: Unsicherheit und Stillstand, Bonn.

Mueller, D.C. (1989): Public Choice II, erw. Aufl., Cambridge.

National Economic Research Associates (NERA) (1998): Estimationg the Long Run Incremental Cost of PSTN Access, A Draft Report for ACCC, London, October 1998.

Needy, C.W. (1997): Untangling Tin Cans on a String: The Difficulty of Regulating Access to even the Simplest Telephone Exchange, in: The Yale Journal on Regulation, Bd. 14, S. 85-144.

Nett, L. (1998): Der Shapley-Wert. Ein Allokationsmechanismus für die Zuordnung von Gemeinkosten, in: Das Wirtschaftsstudium, Heft 10, S. 525-528.

Neumann, K.H. (1999): Wettbewerb im Ortsnetz, in: Telekom Praxis, Heft 4, S. 21-27.

Niskanen, W.A. (1968): Nonmarket Decision Making: The Peculiar Economics of Bureaucracy, in: American Economic Review, Bd. 58, S. $293 \mathrm{ff}$.

Niskanen, W.A. (1971): Bureaucracy and Representative Government, Chicago.

Niskanen, W.A. (1975): Bureaucrats and Politicians, in: The Journal of Law and Economics, Bd. 18, S. 617 ff.

Nortelnetworks: http://www.nortelnetworks.com/cool/lnp/survey.html

Noth, M. (1994): Regulierung bei asymmetrischer Informationsverteilung, Wiesbaden. 
Office of Telecommunications (Oftel) (1994): A Framework for Effective Competition, London.

Office of Telecommunications (Oftel) (1996a): The Methodology to Calculate Long Run Incremental Costs, NERA Report, London.

Office of Telecommunications (Oftel) (1996b): Reconciliation of the Top Down and Bottom Up Incremental Costs Models, NERA Report, London: Office of Telecommunications, June 1996.

Office of Telecommunications (Oftel) (1997a): Long Run Incremental Costs Methodology, British Telecom Report, London, November 1997.

Office of Telecommunications (Oftel) (1997b): An Assessment of the 1995/96 Top-Down Model, NERA Report, London.

Office of Telecommunications (Oftel) (1997c): An Assessment of the 1996/97 Top-Down Model, NERA Report, London.

Office of Telecommunications (Oftel) (1998): Effective Competition Review, Statement, London.

Office of Telecommunications (Oftel) (1999): Determination of Final Charges for BT's Standard Services from 1 April to 30 September 1997, London.

Olson, M. (1965): The Logic of Collective Action, Cambridge.

Panzar, J. (1998): Technological Determinants of Firm and Industry Structure, in: R. Schmalensee, R.D. Willig (Hrsg.), Handbook of Industrial Organization, 5. Aufl., Amsterdam.

Picot, A. (1991): Ökonomische Theorien der Organisation- Ein Überblick über neuere Ansätze und deren betriebswirtschaftliches Anwendungspotential, in: D. Orgelheide, B. Rudolph, E. Büsselmann (Hrsg.), Betriebswirtschaftslehre und ökonomische Theorie, Stuttgart, S. 143-170.

Pfaff, D. (1994): On the Allocation of Overhead Costs, in: European Accounting Review, Bd. 3, S. 49-70. 
Pfaff, D. (1996): Kostenrechnung als Instrument der Entscheidungssteuerung. Chancen und Probleme, in: Kostenrechnungspraxis, 40. Jg., S. 151-156.

Pfaff, D.; Zweifel, P. (1998): Die Principal-Agent Theorie. Ein fruchtbarer Beitrag der Wirtschaftstheorie zur Praxis, in: Das Wirtschaftsstudium, Heft 4, S.184-190.

Pfähler, W.; Wiese, H. (1991): Produktdifferenzierung im Oligopol, in: Das Wirtschaftsstudium, Heft 6, S. 459-463.

Plum, M.; Schwarz-Schilling, C. (2000): Die Abgrenzung des relevanten Marktes im Telekommunikations- und Postbereich, WIK Diskussionsbeitrag Nr. 200; Bad Honnef.

Proebster, W. (1998): Rechnernetze: Technik, Protokolle, Systeme, Anwendungen, München.

Probe Research (1998): White-Paper: The Future of Online Communication, unter www.proberesearch.com

Rabe, U. (1989): Verbundproduktion von Telekommunikationsdiensten und das Problem der Kostenzurechnung, in: Archiv furr das Post- und Fernmeldewesen, 41. Jg., Heft 2, S. 147-162.

Ramsey, F.P. (1927): A Contribution to the Theory of Taxation, in: The Economic Journal, Bd. 37, Heft 145, S. 47-61.

Rathgeb, E.; Wallmeier, E. (1997): ATM - Infrastruktur für die Hochleistungskommunikation, Berlin.

Rathgeb, E. (1998): Telekommunikationsnetze der Zukunft, in: Telekom Praxis, Heft 9, S. 19-28.

Regio Net AG (1998): Pressemitteilung vom 16.09.1998.

Regulierungsbehörde für Telekommunikation und Post (RegTP): Pressemitteilungen vom 25.08.1999, 08.09.1999, 22.01.2002, 12.02.2002, 03.06.2002, 12.04.2002 unter http://www.regtp.de/ 
Regulierungsbehörde für Telekommunikation und Post (RegTP): Amtsblatt 16/2000; Mitteilung 507/00; 24/00; 738/00; 5/2001; 122/2001.

Regulierungsbehörde für Telekommunikation und Post (RegTP) (2000): Jahresbericht 2000, Marktbeobachtungsdaten der Regulierungsbehörde furr Telekommunikation und Post, Bonn.

Regulierungsbehörde für Telekommunikation und Post (2001a): Ortsnetzwettbewerb 2000, Situationsbericht zum deutschen Ortsnetzwettbewerb, Bonn.

Regulierungsbehörde für Telekommunikation und Post (2001b): 2. Tätigkeitsbereicht fur die Jahre 2000/2001, Bonn.

Reinke, T.H. (1998): Local Number Portability and Local Loop Competition, in: Telecommunications Policy, Bd. 22, Heft 1, S. 73-87.

Robinson; J (1969): The Economics of Imperfect Competition, 2. Aufl., London, New York.

Rohlfs, J. (1974): A Theory of Interdependent Demand for Communication Service, in: Bell Journal of Economis, Bd. 5, S. 16-37.

Rosenbaum, O. (1998): ISDN-Fachlexikon, 2. Aufl., Kaarst.

Roth, A.E. (1988): Introduction to the Shapley Value, in: A.E. Roth (Hrsg.), The Shapley-Value. Essays in Honour of Lloyd Shapley, Cambridge, S. 1-27.

Rothschild, M.; Stiglitz, J.E. (1976): Equilibrium in Competitive Insurance Markets: An Essay on the Economics of Imperfect Information, in: Quarterly Journal of Economics, Bd. 90, S. 629-649.

Rottenbiller, S. (2002): Essential Facilities als ordnungspolitisches Problem, Hamburg.

Rühl, J. (2001): Vertragliche Gestaltung von Innovationskooperationen, Wiesbaden.

Scitovski, T. (1954): Two Concepts of External Economies, in: Journal of Political Economy, Bd. 62, Heft 2, S.143-151. 
Schmidt, I. (1996): Wettbewerbspolitik und Kartellrecht, 5. Aufl., Stuttgart.

Schmoll, S.; Wist, B. (1997): Neue technische Entwicklungen im Teilnehmerzugangsbereich, in: H. Kubicek u.a. (Hrsg.), Jahrbuch Telekommunikation und Gesellschaft, Bd. 5: Die Ware Information- auf dem Weg zu einer Informations-ökonomik, Heidelberg, S. 312 und Volltext auf Diskettendatei Wiest.rtf

Schneider, E. (1972): Einführung in die Wirtschaftstheorie - II. Teil, 13. Aufl., Tübingen.

Schütz, R.; Müller, R.-G. (1999): Entgeltregulierung in der Telekommunikation, in: MultiMedia und Recht, Bd. 2; Heft 3, S. 128-137.

Schïtz, R. (2001): Anmerkung zu OVG Münster: Zusammenschaltungsanordnung bei gleichzeitiger Festsetzung der Zusammenschaltungsentgelte , in: MultiMedia und Recht, Bd. 4, Heft 8, S. 552-555.

Schwartz, M. (1996): Telecommunications Reform in the US: Promises and Pitfalls, in: Econ WPA, ewp-io / 9607001.

Shapley, L. (1953): A Value for n-Person Games, in: H.W. Kuhn, A.W. Tucker (Hrsg.): Contributions to the Theory of Games, Bd. 2, Princeton, S. 307-317.

Sharkey, W.W. (1982): The Theory of Natural Monopoly, Cambridge, Mass.

Shepherd, W.G. (1984): Contestability versus Competition, in: American Economic Review, Bd. 74, S. 572-587.

Sidak, G.J.; Spulber, D.F. (1998a): Deregulatory Takings and the Regulatory Contract, Cambridge, Mass.

Sidak, G.J.; Spulber, D.F. (1998b): Deregulation and Managed Competition in Network Industries, in: The Yale Journal on Regulation, Bd. 15, S. 117-147.

Siegmund, G. (1999): Technik der Netze, 4. Aufl., Heidelberg.

Sietmann, R. (1997): Die Zukunft im Local Loop, in: Funkschau, Bd. 7, S. 34-42.

Sietmann, R. (1999): Durchs Abwasser zum Kunden, in: Funkschau, Bd. 1/2, S. 60-62. 
Slater, M. (1989): The Rationale for Marginal Cost Pricing, in: D. Helm, J. Kay, D. Thomposon (Hrsg.), The Market for Energy, Oxford, S. 133-154.

Spence, M.; Zeckhauser, R. (1971): Insurance, Information, and Individual Action, in: American Economic Review, Bd. 61, S. 380-387.

Stackelberg, H. v. (1934): Marktform und Gleichgewicht, Wien, Berlin.

Stamm, P. (2000): Entwicklungsstand und Perspektiven von Powerline Communication. Diskussionsbeitrag Nr. 201 des Wissenschaftlichen Instituts für Kommunikationsdienste, Bad Honnef.

Stumpf, U.; Schwarz-Schilling, C. (1999): Wettbewerb auf Telekommunikationsmärkten, WIK Diskussionsbeitrag Nr. 197, Bad Honnef.

Thum, M. (1994): Network Externalities, Technological Progress, and the Competition of Market Contracts, in: International Journal of Industrial Organisation, Bd. 12, Heft 2, S. 269-289.

Thum, M. (1995): Netzwerkeffekte, Standardisierung und staatlicher Regulierungsbedarf, Tübingen.

Tirole, J. (1995): Industrieökonomik, München.

Train, K.E. (1991): Optimal Regulation. The Economic Theory of Natural Monopoly, Cambridge, Mass.

Triffin, R. (1940): Monopolistic Competition and General Equilibrium Theory, Cambridge, Mass.

Tullock, G. (1967): The Welfare Costs of Tariffs, Monopolies and Theft, in: Western Economic Journal, Bd. 5, S. 224-32.

Tye, W.B. (1994): The Pricing of Inputs sold to the Competitors: A Response, in: The Yale Journal on Regulation, Bd. 11, S. 203-224.

Varian, H. (1991): Grundzüge der Mikroökonomik, 2. Aufl., München. 
Valletti, T. (1999): The Practice of Access Pricing: Telecommunications in the United Kingdom, Policy Research Paper.Nr. 2063, The World Bank, Economic Development Institute.

Valletti; T.; Estache A. (1999): The Theory of Access Pricing: An Overview for Infrastructure Regulators. Diskussionspapier Nr. 2133 of the CEPR, London.

VATM (2001): Jahresbericht - Überblick über die Entwicklung im deutschen Telekommunikationsmarkt im Jahre 2001, Köln. http://www.vatm.de.

Veröffentlichungskommission des OVG Münster (2001): Zusammenschaltungsanordnung bei gleichzeitiger Festsetzung der Zusammenschaltungsentgelte, in: MultiMedia und Recht, Bd. 4, Heft 8, S. 548-551.

Vogelsang, I. (1996a): Deregulierung und Infrastruktur im Bereich der Telekommunikation in den USA, in: Wettbewerb und Infrastruktur in Post- und Telekommunikationsmärkten, Baden-Baden, S. 137-149.

Vogelsang, I. (1996b): Kosten des Ortsnetzes, Studie des Verbands der Telekommunikationsnetz- und Mehrwertdiensteanbieter (VTM), Hürth.

Vogelsang, I. (1996c): Wettbewerb im Ortsnetz - Neue Entwicklungen in den USA, WIK-Diskussionsbeitrag Nr. 168, Bad Honnef.

Vogelsang, I. (1998): Analytische Kostenmodelle - ein notwendiges Übel, in: MultiMedia und Recht, Bd. 1, Heft 11, S. 594-598.

VPRT (Hrsg.) (1997): Entwicklung der BK-Netze in Deutschland - Teil 1 Kosten- und Strukturanalyse, Berlin.

VPRT (Hrsg.) (1999): Entwicklung der BK-Netze in Deutschland - Teil 2 Wirtschaftliche Situation und zukünftige Einflußfaktoren, Berlin.

Wey, C. (1999): Marktorganisation durch Standardisierung: Ein Beitrag zur Neuen Institutionenökonomik des Marktes, Berlin.

Weizsăcker, C.C.v. (1997): Wettbewerb in Netzen, in: Wirtschaft und Wettbewerb, 47. Jahrgang, S. 572-79. 
Wickert-Nick, D.v. (1999): Wettbewerb im lokalen Telekommunikationsmarkt, Baden-Baden.

Wiese, H. (1990): Netzeffekte und Kompatibilität, Stuttgart.

Willig, R.D. (1979): The Theory of Network Access Pricing, in: H.M. Trebing (Hrsg.), Issues in Public Utility Regulation: Proceedings of the Institute of Public Utilities Tenth Annual Conference, Michigan State University Public Utilities Paper, East Lansing, Michigan.

Willig, R.D. (1980): What can Markets Control?, in: R. Sherman (Hrsg.), Perspectives on Postal Services Issues, Washington, S. 137-159.

Windisch, R. (1987): Privatisierung natürlicher Monopole: Theoretische Grundlagen und Kriterien, in: R. Windisch (Hrsg.), Privatisierung natürlicher Monopole im Bereich Bahn, Post und Telekommunikation, Tübingen.

Winzer, P. (2000): Kostenorientierte Bestimmung von Preisen für den Zugang zu Telekommunikationsnetzen marktbeherrschender Unternehmen, Frankfurt a.M. u.a.

Wissenschaftliches Institut für Kommunikationsdienste (WIK) (1998): Ein analytisches Kostenmodell für das Ortsnetz, Referenzdokument.

Wissenschaftliches Institut für Kommunikationsdienste (WIK) (1999): Ein analytisches Kostenmodell für das nationale Verbindungsnetz, Referenzdokument.

Wissenschaftliches Institut für Kommunikationsdienste/European-American Center for Political Analysis (WIK/ EAC) (1994): Network Interconnection in the Domain of ONP; Study for DGXIII of the European Commission, Final Report, Bad Honnef.

Woeckener, B. (1994): Dynamische Marktprozesse bei Netzwerkexternalitäten und begrenzter Rationalităt, in: A.E. Ott (Hrsg.), Probleme der unvollkommenen Konkurrenz, Tübingen.

Zimmermann, M.; Dostert, K. (1998): Sprache uber die Stromleitung, in: Funkschau, Heft 4, S. 22-27. 
Gesetzes- und Verordnungstexte der Bundesrepublik Deutschland

Gesetz gegen Wettbewerbsbeschränkungen [GWB] (1990) vom 20.02.1990, veröffentlicht in: Bundesgesetzblatt v. 28.02.1990, Teil I, Nr. 7, S. 235.

Telekommunikations-Entgeltregulierungsverordnung [TentgV] (1996) vom 01.10.19996, veröffentlicht in: Bundesgesetzblatt v. 09.10.1996, Teil I, Nr. 49; S.1492.

Telekommunikationsgesetz [TKG] (1996) vom 25.07.1996, veröffentlicht in: Bundesgesetzblatt v. 31.07.1996, Teil I, Nr. 39; S. 1120.

Verordnung luber besondere Netzzugänge/Netzzugangsverordnung [NZV] (1996) vom 23.10.1996, veröffentlicht in: Bundesgesetzblatt v. 31.10.1996, Teil I, Nr. 53, S. 1568. 


\title{
SCHRIFTEN ZUR WIRTSCHAFTSTHEORIE UND WIRTSCHAFTSPOLITIK
}

\author{
Herausgegeben von Rolf Hasse, Jörn Kruse, Wolf Schäfer, \\ Thomas Straubhaar, Klaus W. Zimmermann
}

Band 1 Lars Bünning: Die Konvergenzkriterien des Maastricht-Vertrages unter besonderer Berücksichtlgung ihrer Konsistenz. 1997.

Band 2 Andreas Henning: Beveridge-Kurve, Lohnsetzung und Langzeitarbeitslosigkeit. Eine theoretische Untersuchung unter Berücksichtigung des Insider-Outsider-Ansatzes und der Entwertung des Humankapitals. 1997.

Band 3 Iris Henning: Die Reputation einer Zentralbank. Eine theoretische Untersuchung unter besonderer Berücksichtigung der Europăischen Zentralbank. 1997.

Band 4 Rüdiger Hermann: Ein gemeinsamer Markt für Elektrizität in Europa. Optionen einer Wettbewerbsordnung zwischen Anspruch und Wirklichkeit. 1997.

Band 5 Alexander Tiedtke: Japan und der Vorwurt des Trittbrettfahrerverhaltens in der US-amerikanisch-japanischen Allianz. 1997.

Band 6 Wolfgang Grimme: Ordnungspolitisches Konzept der Regionalpolitik. Darstellung der Defizite und des Reformbedarts der Regionalpolitik am Beisplel Mecklenburg-Vorpommems. 1997.

Band 7 Christian Ricken: Determinanten der Effektivität der Umweltpolitik. Der nationale Politikstil im Spannungsfeld von Ökonomie, Politik und Kultur. 1997.

Band 8 Christian Schmidt: Real Convergence in the European Union. An Empirical Analysis. 1997.

Band 9 Silvia Marengo: Exchange Rate Policy for MERCOSUR: Lessons from the European Union. 1998.

Band 10 Jens Kleinemeyer: Standardisierung zwischen Kooperation und Wettbewerb. Eine spieltheoretische Betrachtung. 1998.

Band 11 Stefan M. Golder: Migration und Arbeitsmarkt. Eine empirische Analyse der Performance von Ausländem in der Schweiz. 1999.

Band 12 Stefan Kramer: Die Wirkung einer Internationalisierung des Yen auf die japanischen Finanzmärkte, die japanische Geldpolitik und die Usancen der Fakturierung. 1999.

Band 13 Antje Marielle Gerhold: Wirtschaftliche Integration und Kooperation im asiatisch-pazifischen Raum. Die APEC. 1999.

Band 14 Tamim Achim Dawar: Deutsche Direktinvestitionen in Australien. Eine Evaluation der theoretischen Erklärungsensätze und der Standortattraktlvität des Produktions- und Imvestitionsstandortes Australien. 1999.

Band 15 Hans-Markus Johannsen: Dle ordnungspolitische Haltung Frankreichs im Prozeß der europảischen Einigung. 1999.

Band 16 Annette Schönherr: Vereinigungsbedingte Dimensionen regionaler Arbeitsmobilität. Wirkungen unter analytisch einfachen Bedingungen und potentielle individuelle Migrationsgewinne in Deutschland nach der Vereinigung. 1999.

Band 17 Henrik Müller. Wechselkurspolitik des Eurolandes. Konfliktstoff für die neue währungspolitische Ära. 1999.

Band 18 Lars H. Wengorz: Die Bedeutung von Unternehmertum und Eigentum für die Existenz von Unternehmen. Eine methodenkritische Analyse der Transformation des Wirtschaftssystems in Russland. 2000.

Band 19 Eckart Bauer: Konzeptionelle Grundfragen eines Kinderleistungsausgleichs im Rahmen einer umlagefinanzierten zwangsweisen Rentenversicherung. 2000. 
Band 20 Hubertus Hille: Enlarging the European Union. A Computable General Equilibrium Assessment of Different Integration Scenarios of Central and Eastern Europe. 2001.

Band 21 Tobias Just: Globalisierung und Ideologie. Eine Analyse der Existenz und Persistenz von Partisaneffekten bei zunehmender Internationalisierung der Märkte. 2001.

Band 22 Simone Claber. Großbritannien und die Europäische Integration unter besonderer Berücksichtigung ordnungspolitischer Aspekte. 2002.

Band 23 Silvia Rottenbiller. Essential Facilities als ordnungspolitisches Problem. 2002.

Band 24 Peggy Dreyhaupt von Speicher: Die Regionen Polens, Ungams und der Tschechischen Republik vor dem EU-Beitritt. Interregionale Disparităten, Bestimmungsfaktoren und Lösungsansätze. 2002.

Band 25 Gerhard Rösl: Seigniorage in der EWU. Eine Analyse der Notenbankgewinnentstehung und -verwendung des Eurosystems. 2002.

Band 26 Jörn Quitzau: Die Vergabe der Fernsehrechte an der Fußball-Bundesliga. Wohlfahrtsökonomische, wettbewerbspolitische und sportökonomische Aspekte der Zentralvermarktung. 2003.

Band 27 Małgorzata Stankiewicz: Die polnische Telekommunikation vor dem EU-Beitritt. 2003.

Band 28 Sarah Schnlewindt: Einführung von Wettbewerb auf der Letzten Meile. Eine ökonomische Analyse von Netzinfrastruktur und Wettbewerbspotential im Teilnehmeranschlussbereich. 2003. 